\title{
UNCONVENTIONAL ANIMAL MODELS IN INFECTIOUS DISEASE RESEARCH
}

EDITED BY: Gheyath Khaled Nasrallah, Layla Kamareddine and Buket Baddal PUBLISHED IN: Frontiers in Cellular and Infection Microbiology

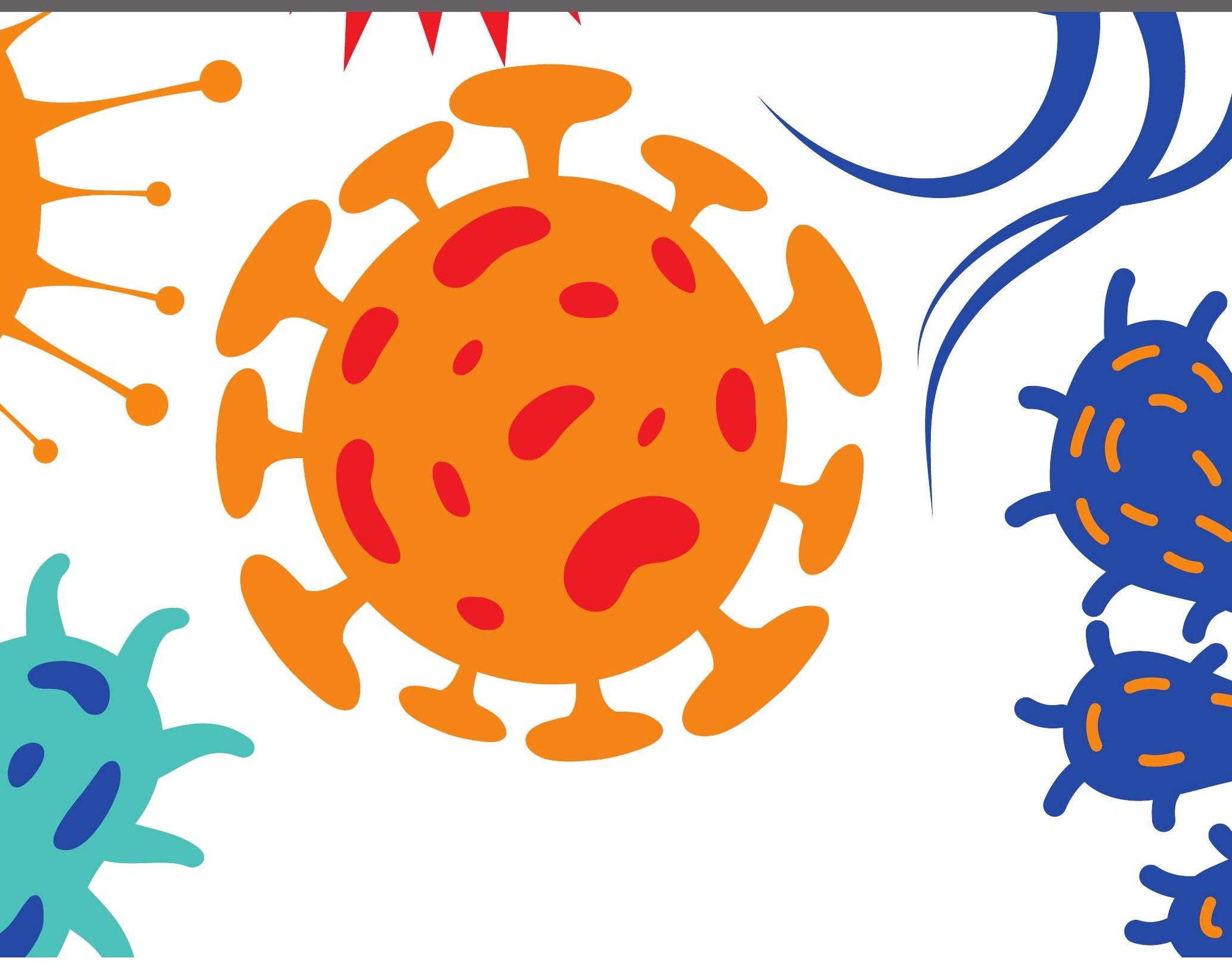




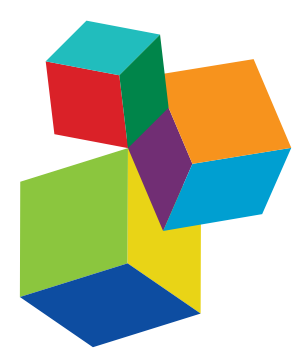

Frontiers eBook Copyright Statement

The copyright in the text of individual articles in this eBook is the property of their respective authors or their respective institutions or funders. The copyright in graphics and images within each article may be subject to copyright of other parties. In both cases this is subject to a license granted to Frontiers.

The compilation of articles constituting this eBook is the property of Frontiers.

Each article within this eBook, and the eBook itself, are published under the most recent version of the Creative Commons CC-BY licence.

The version current at the date of publication of this eBook is CC-BY 4.0. If the CC-BY licence is updated, the licence granted by Frontiers is automatically updated to the new version.

When exercising any right under the CC-BY licence, Frontiers must be attributed as the original publisher of the article or eBook, as applicable.

Authors have the responsibility of ensuring that any graphics or other materials which are the property of others may be included in the

CC-BY licence, but this should be checked before relying on the

CC-BY licence to reproduce those materials. Any copyright notices relating to those materials must be complied with.

Copyright and source acknowledgement notices may not be removed and must be displayed in any copy, derivative work or partial copy which includes the elements in question.

All copyright, and all rights therein, are protected by national and international copyright laws. The above represents a summary only.

For further information please read Frontiers' Conditions for Website

Use and Copyright Statement, and the applicable CC-BY licence.

ISSN 1664-8714

ISBN 978-2-88971-890-0

DOI 10.3389/978-2-88971-890-0

\section{About Frontiers}

Frontiers is more than just an open-access publisher of scholarly articles: it is a pioneering approach to the world of academia, radically improving the way scholarly research is managed. The grand vision of Frontiers is a world where all people have an equal opportunity to seek, share and generate knowledge. Frontiers provides immediate and permanent online open access to all its publications, but this alone is not enough to realize our grand goals.

\section{Frontiers Journal Series}

The Frontiers Journal Series is a multi-tier and interdisciplinary set of open-access, online journals, promising a paradigm shift from the current review, selection and dissemination processes in academic publishing. All Frontiers journals are driven by researchers for researchers; therefore, they constitute a service to the scholarly community. At the same time, the Frontiers Journal Series operates on a revolutionary invention, the tiered publishing system, initially addressing specific communities of scholars, and gradually climbing up to broader public understanding, thus serving the interests of the lay society, too.

\section{Dedication to Quality}

Each Frontiers article is a landmark of the highest quality, thanks to genuinely collaborative interactions between authors and review editors, who include some of the world's best academicians. Research must be certified by peers before entering a stream of knowledge that may eventually reach the public - and shape society; therefore, Frontiers only applies the most rigorous and unbiased reviews.

Frontiers revolutionizes research publishing by freely delivering the most outstanding research, evaluated with no bias from both the academic and social point of view. By applying the most advanced information technologies, Frontiers is catapulting scholarly publishing into a new generation.

\section{What are Frontiers Research Topics?}

Frontiers Research Topics are very popular trademarks of the Frontiers Journals Series: they are collections of at least ten articles, all centered on a particular subject. With their unique mix of varied contributions from Original Research to Review Articles, Frontiers Research Topics unify the most influential researchers, the latest key findings and historical advances in a hot research area! Find out more on how to host your own Frontiers Research Topic or contribute to one as an author by contacting the Frontiers Editorial Office: frontiersin.org/about/contact 


\section{UNCONVENTIONAL ANIMAL MODELS IN INFECTIOUS DISEASE RESEARCH}

Topic Editors:

Gheyath Khaled Nasrallah, Qatar University, Qatar Layla Kamareddine, Qatar University, Qatar

Buket Baddal, Near East University, Cyprus

Citation: Nasrallah, G. K., Kamareddine, L., Baddal, B., eds. (2021). Unconventional Animal Models in Infectious Disease Research. Lausanne: Frontiers Media SA. doi: 10.3389/978-2-88971-890-0 


\section{Table of Contents}

05

Editorial: Unconventional Animal Models in Infectious Disease Research Layla Kamareddine

08 Editorial: Unconventional Animal Models in Infectious Disease Research - Part I

Nadin Younes and Gheyath K. Nasrallah

12 Duplicate US1 Genes of Duck Enteritis Virus Encode a Non-essential Immediate Early Protein Localized to the Nucleus

Yangguang Li, Ying Wu, Mingshu Wang, YunChao Ma, Renyong Jia, Shun Chen, Dekang Zhu, Mafeng Liu, Qiao Yang, Xinxin Zhao, Shaqiu Zhang, Juan Huang, Xumin Ou, Sai Mao, Ling Zhang, Yunya Liu, Yanling Yu, Leichang Pan, Bin Tian, Mujeeb Ur Rehman, Xiaoyue Chen and Anchun Cheng

25 Autophagy Is a Potential Therapeutic Target Against Duck Tembusu Virus Infection in vivo

Zhiqiang Hu, Yuhong Pan, Anchun Cheng, Xingcui Zhang, Mingshu Wang, Shun Chen, Dekang Zhu, Mafeng Liu, Qiao Yang, Ying Wu, Xinxin Zhao, Juan Huang, Shaqiu Zhang, Sai Mao, Xumin Ou, Yanling Yu, Ling Zhang, Yunya Liu, Bin Tian, Leichang Pan, Mujeeb Ur Rehman, Zhongqiong Yin and Renyong Jia

38 Corrigendum: Autophagy Is a Potential Therapeutic Target Against Duck Tembusu Virus Infection in Vivo

Zhiqiang Hu, Yuhong Pan, Anchun Cheng, Xingcui Zhang, Mingshu Wang, Shun Chen, Dekang Zhu, Mafeng Liu, Qiao Yang, Ying Wu, Xinxin Zhao, Juan Huang, Shaqiu Zhang, Sai Mao, Xumin Ou, Yanling Yu, Ling Zhang, Yunya Liu, Bin Tian, Leichang Pan, Mujeeb Ur Rehman, Zhongqiong Yin and Renyong Jia

40 Drosophila as a Model Organism in Host-Pathogen Interaction Studies Salma Younes, Asma Al-Sulaiti, Elham Abdulwahab Ahmed Nasser, Hoda Najjar and Layla Kamareddine

56 CFTR Depletion Confers Hypersusceptibility to Mycobacterium fortuitum in a Zebrafish Model

Matt D. Johansen and Laurent Kremer

70 CLIPB10 is a Terminal Protease in the Regulatory Network That Controls Melanization in the African Malaria Mosquito Anopheles gambiae

Xin Zhang, Miao Li, Layla El Moussawi, Sally Saab, Shasha Zhang,

Mike A. Osta and Kristin Michel

83 Galleria mellonella Larvae as an Infection Model to Investigate sRNA-Mediated Pathogenesis in Staphylococcus aureus

Guillaume Ménard, Astrid Rouillon, Gevorg Ghukasyan, Mathieu Emily,

Brice Felden and Pierre-Yves Donnio 
95 Development and Characterization of High-Throughput Caenorhabditis elegans - Enterococcus faecium Infection Model

Alexey V. Revtovich, Elissa Tjahjono, Kavindra V. Singh, Blake M. Hanson, Barbara E. Murray and Natalia V. Kirienko

110 Exogenous Autoinducer-2 Rescues Intestinal Dysbiosis and Intestinal Inflammation in a Neonatal Mouse Necrotizing Enterocolitis Model

Yan-Chun Ji, Qian Sun, Chun-Yan Fu, Xiang She, Xiao-Chen Liu, Yu He, Qing Ai, Lu-Quan Li and Zheng-Li Wang 
Editorial: Unconventional Animal Models in Infectious Disease Research

\author{
Layla Kamareddine ${ }^{1,2,3 *}$ \\ 1 Department of Biomedical Sciences, College of Health Sciences, QU Health, Qatar University, Doha, Qatar, ${ }^{2}$ Biomedical \\ Research Center, Qatar University, Doha, Qatar, ${ }^{3}$ Biomedical and Pharmaceutical Research Unit, QU Health, Qatar \\ University, Doha, Qatar
}

Keywords: unconventional animal models, host-pathogen interactions, infectious disease research, treatment targets, in vivo studies

Editorial on the Research Topic

Unconventional Animal Models in Infectious Disease Research

\section{OPEN ACCESS}

Edited and reviewed by: Thomas Rudel,

Julius Maximilian University of

Würzburg, Germany

*Correspondence:

Layla Kamareddine

Ikamareddine@qu.edu.qa

Specialty section:

This article was submitted to

Bacteria and Host,

a section of the journal

Frontiers in Cellular and

Infection Microbiology

Received: 22 August 2021 Accepted: 11 October 2021 Published: 26 October 2021

Citation:

Kamareddine L (2021) Editorial: Unconventional Animal Models in Infectious Disease Research.

Front. Cell. Infect. Microbiol. 11:762581.

doi: 10.3389/fcimb.2021.762581
Beyond an in vitro setting, the use of a biological system is considered imperious to unravel the enigma of host-pathogen interactions, particularly those eventuating in an infectious disease scenery. In the past two-decades, the use of animal models, especially the unconventional ones, in studying infectious disease occurrence and progression has stemmed out. This rise in the use of animal models in research was greatly reinforced by the furtherance advancements in the field of genetics that has opened up for feasible genetic manipulation of both the host and the pathogen as needed throughout the course of conducted studies. Within this frame of reference, our launched topic envisioned to bring in research work that have used a broad spectrum of unconventional animal models to introduce pioneering findings in host-pathogen interaction studies. These findings will not only shed more light on our understanding of host-pathogen interfaces, but also set the foundation for innovative therapeutic regimens to control infectious diseases.

In the first contributed article to our Research Topic (Li et al.), the authors described the characteristics of the duck enteritis virus (DEV) ICP22 protein and explored its role in DEV replication. Their findings present ICP22 as a non-essential immediate early protein chiefly located in the nucleus of infected duck embryo fibroblasts cells and reveal that ICP22 encloses a classical nuclear localization signal at 305-314 AA, with a need of residue 309 for ICP22 nuclear localization. Moreover, their verdicts evidently highlight the role of the US1 gene encoding ICP22 in DEV replication. One potential benefit of this finding could be implicated in combating Duck plague infectious disease caused by DEV.

Within the poultry viral infectious disease contexture as well, and as part of the ongoing search for novel anti-viral control strategies, the second contributed article to our Research Topic ( $\mathrm{Hu}$ et al.) present autophagy as a potential therapeutic target for Duck tembusu virus (DTMUV) infection. In this study conducted on ducks as the animal model of choice, and as a follow up on their previous in vitro work divulging that autophagy promotes DTMUV replication (Hu et al., 2020), the authors show that autophagy is triggered in a DTMUV infection setting and that autophagy inhibitors impedes DTMUV replication and attenuates DTMUV-caused pathological manifestations possibly through a host innate immune response-dependent manner. 
Owing to the importance of using Drosophila melanogaster in infectious disease research and to our recently achieved groundbreaking findings in host-pathogen interaction studies using the fruit fly model organism (Kamareddine et al., 2018a; Kamareddine et al., 2018b; Jugder et al., 2021), the third contribution to this Research Topic was a review put up by our group to distinctly highlight the importance and advantages of Drosophila as a model organism of choice in host-pathogen interactions studies (Younes et al.). Communally, this review, along with others including another review from our group on a similar subject matter (Kamareddine et al., 2020) aim to provide comprehensive insight into the use of animal models in disease research.

Besides Drosophila, zebrafish also gained great popularity in infectious disease research as a prime model organism. Several studies including that of Johansen and Kremer submitted to our Research Topic, for example, employed the use of zebrafish embryos to unravel the pathogenesis of human diseases. In their study herein, the authors investigated the disease pathogenesis entangled in Mycobacterium fortuitum infection and revealed a role of the cystic fibrosis transmembrane conductance regulator (CFTR) in host immune defense against this pulmonary and extra-pulmonary infection-causing bacteria.

Within the same notion of implementing animal models to decipher key players involved in immune defense mechanisms against invading pathogens, Zhang et al.'s study in this Research Topic exploited the Anopheles gambiae mosquito model, along with genetic and biochemical assays, to underline the role of the clip domain serine protease CLIPB10 in the regulatory network controlling the melanization immune response against the malaria parasites. The findings of this study acquaints previous similar work from this research group on the Anopheles gambiae model organism, mainly those directed towards unraveling the molecular mechanisms of different innate immune arms in the malaria vector against invading pathogens (Yassine et al., 2012; Yassine et al., 2014; Kamareddine et al., 2016; Zhang et al., 2016; El Moussawi et al., 2019).

Lately, the greater wax moth, Galleria mellonella, has been also introduced as a new-fangled model for host-pathogen interaction studies (Bismuth et al., 2019). In their contributed work to this Research Topic, Ménard et al. present larval staged

\section{REFERENCES}

Bismuth, H., Aussel, L., and Ezraty, B. (2019). La Teigne Galleria Mellonella Pour Les Études Hôte-Pathogène [The Greater Wax Moth, Galleria Mellonella to Study Host-Pathogen Interactions]. Med Sci. M/S 35 (4), 346-351. doi: 10.1051/medsci/2019071

El Moussawi, L., Nakhleh, J., Kamareddine, L., and Osta, M. A. (2019). The Mosquito Melanization Response Requires Hierarchical Activation of nonCatalytic Clip Domain Serine Protease Homologs. PloS Pathog. 15 (11), e1008194. doi: 10.1371/journal.ppat.1008194

Hu, Z., Pan, Y., Cheng, A., Zhang, X., Wang, M., Chen, S., et al. (2020). Autophagy Promotes Duck Tembusu Virus Replication by Suppressing P62/SQSTM1Mediated Innate Immune Responses In Vitro. Vaccines 8 (1), 22. doi: 10.3390/ vaccines 8010022

Jugder, B. E., Kamareddine, L., and Watnick, P. I. (2021). Microbiota-Derived Acetate Activates Intestinal Innate Immunity via the Tip60 Histone Acetyltransferase Complex. Immunity 54 (8), 1683-1697.e3. doi: 10.1016/j.immuni.2021.05.017
Galleria mellonella as a fast and easy system to use for investigating the role of small regulatory RNAs (sRNAs) in staphylococcus aureus virulence, opening up for similar future studies on other human bacterial pathogens using this model organism. Likewise, Revtovich et al.'s contribution to this Research Topic represent the first developed and characterized "high-throughput C. elegans-E. faecium infection model" used for identifying a spectrum of virulence genes that take part in the pathogenesis of the bacterial infection. Such an established platform could be eventually built on to introduce novel treatment approaches for E. faecium infection, fundamentally those emerging in hospital settings.

Although our Research Topic was particularly directed towards the use of unconventional animal models in infectious disease research, mice as traditional model organisms have always served as a basic platform for in vivo studies. As such, we welcomed the contribution of Ji et al. to this Research Topic whose work relied on the use of a neonatal mouse necrotizing enterocolitis (NEC) model to study the effect of exogenous Autoinducer-2 (AI-2) on intestinal dysbiosis and inflammation and therefore clinch a role of AI-2 in partially rescuing flora disruption and decreasing the inflammatory response in the mouse NEC model.

Owing to the outstretched success we achieved in this volume of our Research Topic, we have now launched volume II under the same topic title in which we welcome more submissions and aim to initiate a useful foundation for interested scientists in this subject matter.

\section{AUTHOR CONTRIBUTIONS}

The author confirms being the sole contributor to this editorial and has approved it for publication.

\section{ACKNOWLEDGMENTS}

We acknowledge everyone that has contributed to the success of this volume of our Research Topic.

Kamareddine, L., Najjar, H., Sohail, M. U., Abdulkader, H., and Al-Asmakh, M. (2020). The Microbiota and Gut-Related Disorders: Insights From Animal Models. Cells 9 (11), 2401. doi: 10.3390/cells9112401

Kamareddine, L., Nakhleh, J., and Osta, M. A. (2016). Functional Interaction Between Apolipophorins and Complement Regulate the Mosquito Immune Response to Systemic Infections. J. innate Immun. 8 (3), 314-326. doi: 10.1159/ 000443883

Kamareddine, L., Robins, W. P., Berkey, C. D., Mekalanos, J. J. , and Watnick, P. I. (2018b). The Drosophila Immune Deficiency Pathway Modulates Enteroendocrine Function and Host Metabolism. Cell Metab. 28 (3), 449462.e5. doi: 10.1016/j.cmet.2018.05.026

Kamareddine, L., Wong, A., Vanhove, A. S., Hang, S., Purdy, A. E., KierekPearson, K., et al. (2018a). Activation of Vibrio Cholerae Quorum Sensing Promotes Survival of an Arthropod Host. Nat. Microbiol. 3 (2), 243-252. doi: 10.1038/s41564-017-0065-7

Yassine, H., Kamareddine, L., Chamat, S., Christophides, G. K., and Osta, M. A. (2014). A Serine Protease Homolog Negatively Regulates TEP1 Consumption 
in Systemic Infections of the Malaria Vector Anopheles Gambiae. J. Innate Immun. 6 (6), 806-818. doi: 10.1159/000363296

Yassine, H., Kamareddine, L., and Osta, M. A. (2012). The Mosquito Melanization Response is Implicated in Defense Against the Entomopathogenic Fungus Beauveria Bassiana. PloS Pathog. 8 (11), e1003029. doi: 10.1371/journal.ppat.1003029

Zhang, X., An, C., Sprigg, K., and Michel, K. (2016). CLIPB8 is Part of the Prophenoloxidase Activation System in Anopheles Gambiae Mosquitoes. Insect Biochem. Mol. Biol. 71, 106-115. doi: 10.1016/j.ibmb.2016.02.008

Conflict of Interest: The author declares that the research was conducted in the absence of any commercial or financial relationships that could be construed as a potential conflict of interest.
Publisher's Note: All claims expressed in this article are solely those of the authors and do not necessarily represent those of their affiliated organizations, or those of the publisher, the editors and the reviewers. Any product that may be evaluated in this article, or claim that may be made by its manufacturer, is not guaranteed or endorsed by the publisher.

Copyright (c) 2021 Kamareddine. This is an open-access article distributed under the terms of the Creative Commons Attribution License (CC BY). The use, distribution or reproduction in other forums is permitted, provided the original author(s) and the copyright owner(s) are credited and that the original publication in this journal is cited, in accordance with accepted academic practice. No use, distribution or reproduction is permitted which does not comply with these terms. 


\title{
Editorial: Unconventional Animal Models in Infectious Disease Research - Part I
}

\author{
Nadin Younes ${ }^{1}$ and Gheyath K. Nasrallah ${ }^{1,2 *}$ \\ ${ }^{1}$ Biomedical Research Center, Qatar University Health, Qatar University, Doha, Qatar, ${ }^{2}$ Department of Biomedical Science, \\ College of Health Sciences, Qatar University Health, Qatar University, Doha, Qatar
}

Keywords: vaccines, therapeutic agents, infectious disease, host-pathogen interactions, animal model

Editorial on the Research Topic

Unconventional Animal Models in Infectious Disease Research

The multiplicity of host-pathogen interactions associated with infectious diseases frequently necessitate the use of a complicated biological system. Therefore, animal models have contributed substantially to unraveling the physiopathology of infectious diseases. The feasibility to manipulate different animal models aids in (i) discovering the role of host or microbial factors in the infection pathogenesis, (ii) understanding different mechanisms of tissue invasion, host defense, pathogen dissemination. This Research Topic, which includes 8 high-quality research papers (7 original research articles, and 1 review), sheds the light on the use of unconventional model as a platform for advancement in infectious disease research and address novel ground-breaking findings in host-pathogen interaction studies, which paved the way for discovering new therapeutic targets and vaccine development. The most important findings are summarized in Table 1.

The first article focused on the role of autophagy during duck tembusu virus (DTMUV) infection using duck as a model (Hu et al.). The authors used two drugs as autophagy regulators, which are rapamycin (autophagy enhancer) and methyladenine and chloroquine (autophagy inhibitor). They discovered that DTMUV infection triggered autophagy in duck's spleen and brain. They also demonstrated that the autophagy inhibitors suppressed DTMUV replication and reduced DTMUVinduced pathogenic symptoms. They concluded that autophagic regulation was linked to the expression of innate immunity genes such as the pattern recognition receptors, type I interferons, and cytokines.

The second article also utilized ducks to study the role of DEV ICP22 protein in duck enteritis virus (DEV) (Li et al.). DEV duplicate US1 genes encode a DEV ICP22 protein. The authors reported that ICP22 protein, molecular mass of $57 \mathrm{kDa}$, can enter the nucleus by itself using conventional NLS motif. However, DEV ICP22 protein cannot enter the nucleus after mutating amino acid 309R, demonstrating that this amino acid is the crucial residue for ICP22 localization. Most importantly, they concluded that the DEV ICP22 protein is a non-essential immediate-early protein primarily found in the nucleus of infected duck-embryo-fibroblasts (DEF) cells and that US1 deletion can hamper DEV replication.

Johansen and Kremer were able to show that zebrafish embryos are susceptible to M. fortuitum infection in a dose-dependent manner. Interestingly, they showed that $M$. fortuitum was able to form granulomas in embryos as early as 2 days post-infection. In addition, they noticed that transient 
TABLE 1 | Summary for the outcomes of all accepted articles in this special issue.

\begin{tabular}{|c|c|c|c|c|c|c|}
\hline $\begin{array}{l}\text { Study } \\
\text { type }\end{array}$ & $\begin{array}{c}\text { Organism } \\
\text { Name }\end{array}$ & Animal Model & Aim & $\begin{array}{l}\text { Methods of } \\
\text { assessment }\end{array}$ & Conclusion & Reference \\
\hline $\begin{array}{l}\text { Original } \\
\text { article }\end{array}$ & $\begin{array}{l}\text { DTMUV } \\
\text { virus }\end{array}$ & Duck & $\begin{array}{l}\text { To study the role of } \\
\text { autophagy in facilitating } \\
\text { DTMUV replication "in vivo" }\end{array}$ & $\begin{array}{l}\text { - Western blot (WB) } \\
\text { - Hematoxylin and } \\
\text { eosin staining } \\
\text { - Immunohistochemistry } \\
\text { - } \text { RT-PCR }\end{array}$ & $\begin{array}{l}\text { - DTMUV trigged autophagy, which facilitates } \\
\text { its replication inside the cells and induces } \\
\text { pathological symptoms }\end{array}$ & Hu et al. \\
\hline $\begin{array}{l}\text { Original } \\
\text { article }\end{array}$ & DEV virus & Duck & $\begin{array}{l}\text { To elucidate the roles of } \\
\text { US1 and its NLS in DEV } \\
\text { replication "in vivo" }\end{array}$ & $\begin{array}{l}\text { - RT-PCR } \\
\text { - WB } \\
\text { - Immunofluorescence } \\
\text { assay }\end{array}$ & $\begin{array}{l}\text { - The DEV US1 ORF is } 990 \mathrm{bp} \\
\text { - Molecular mass of the ICP22 protein is 57 } \\
\mathrm{kDa} \\
\text { - ICP22 contains a classical NLS at 305- } \\
312 \mathrm{AA} \text { that is essential for its localization to the } \\
\text { nucleus } \\
\text { - DEV US1 is non-essential for host infection } \\
\text { but associated with a severe growth deficit in } \\
\text { vitro. }\end{array}$ & Li et al. \\
\hline $\begin{array}{l}\text { Original } \\
\text { article }\end{array}$ & $\begin{array}{l}\text { M. } \\
\text { fortuitum }\end{array}$ & Zebrafish & $\begin{array}{l}\text { To study the disease } \\
\text { pathogenesis of } M \text {. } \\
\text { fortuitum infections }\end{array}$ & $\begin{array}{l}\text { - Creation of } \\
\text { fluorescent M. fortuitum } \\
\text { - Morpholino Injection } \\
\text { and CFTR Knockdown } \\
\text { - Zebrafish } \\
\text { Microinjection and } \\
\text { Infection } \\
\text { - Zebrafish live imaging }\end{array}$ & $\begin{array}{l}\text { - Zebrafish embryos form granulomas as early } \\
\text { as } 2 \text { days post-infection } \\
\text { - Transient macrophage depletion in zebrafish } \\
\text { led to rapid embryo death with increased } \\
\text { bacterial extracellular cord formation }\end{array}$ & $\begin{array}{l}\text { Johansen and } \\
\text { Kremer }\end{array}$ \\
\hline $\begin{array}{l}\text { Original } \\
\text { article }\end{array}$ & $\begin{array}{l}\text { G. } \\
\text { mellonella } \\
\text { larvae }\end{array}$ & S. aureus & $\begin{array}{l}\text { To study the function of } \\
\text { sRNAs during S. aureus } \\
\text { infection "in vivo" }\end{array}$ & $\begin{array}{l}\text { - Bacterial growth in the } \\
\text { larvae } \\
\text { - Immunohistochemistry } \\
\text { - Bacterial isolation and } \\
\text { RNA extraction } \\
\text { - Monitoring RNA } \\
\text { expression levels }\end{array}$ & $\begin{array}{l}\text { - G. mellonella larvae is a suitable model to } \\
\text { study sRNA-mediated pathogenesis in S. } \\
\text { aureus } \\
\text { - } \quad \text { sprD and sprC increased during infection } \\
\text { and associated with mortality } \\
\text { - } \quad \text { rnalll expression remained barely detectable } \\
\text { over time }\end{array}$ & Ménard et al. \\
\hline $\begin{array}{l}\text { Original } \\
\text { article }\end{array}$ & P.berghei & A.gambiae & $\begin{array}{l}\text { Study the function of } \\
\text { CLIPB10 in protease } \\
\text { cascades "in vivo" using A. } \\
\text { gambiae as a model }\end{array}$ & $\begin{array}{l}\text { - } \text { RT-PCR } \\
\text { - } \text { Activation of } \\
\text { recombinant zymogens } \\
\text { - } \quad \text { Substrate screening } \\
\text { of active CLIPB10xa } \\
\text { - MALDI-TOF MS } \\
\text { Analysis }\end{array}$ & $\begin{array}{l}\text { - } \quad \text { proPO plays an essential role in the cuticular } \\
\text { melanization in insects } \\
\text { - } \quad \text { CLIPB10 is required for the melanization of } \\
\text { ookinete stages of the rodent malaria parasite } P \text {. } \\
\text { berghei. } \\
\text { - } \quad \text { Recombinant serpin } 2 \text { protein formed a } \\
\text { stable protein complex with CLIPB10 protein }\end{array}$ & Zhang et al. \\
\hline $\begin{array}{l}\text { Original } \\
\text { article }\end{array}$ & C. elegans & E. faecium & $\begin{array}{l}\text { To analyze a panel of lab } \\
\text { strains of E. faecium with } \\
\text { deletions of targeted } \\
\text { virulence factor "in vivo" } \\
\text { using C. elegans as a } \\
\text { model }\end{array}$ & $\begin{array}{l}\text { - C. elegans - } \\
\text { Enterococcus } \\
\text { pathogenesis assays } \\
\text { - Colony forming unit } \\
\text { assay } \\
\text { - Genomic analysis } \\
\text { - Microscopy } \\
\text { - RNA interference } \\
\text { protocol }\end{array}$ & $\begin{array}{l}\text { - C. elegans is a high throughput infection } \\
\text { model for studying the pathogensis of } E \text {. facium } \\
\text { - Removal of certain virulence factors (e.g., } \\
\Delta \text { fms15) was sufficient to affect the virulence of } \\
\text { E. faecium } \\
\text { - Multiple deletions were required to affect } \\
\text { pathogenesis, suggesting that host-pathogen } \\
\text { interactions are multifactorial. }\end{array}$ & Revtovich et al. \\
\hline $\begin{array}{l}\text { Original } \\
\text { article }\end{array}$ & Mouse & $\begin{array}{l}\text { Helicobacter, } \\
\text { Clostridium, } \\
\text { Lactobacillus, } \\
\text { Klebsiella, } \\
\text { Rodentibacter and } \\
\text { Enterococcus }\end{array}$ & $\begin{array}{l}\text { To study the effect of } \\
\text { Autoinducer- } 2 \text { on the } \\
\text { process of necrotizing } \\
\text { enterocolitis mouse model }\end{array}$ & $\begin{array}{l}\text { - Histology } \\
\text { - Immunohistochemistry } \\
\text { - Mouse Intestinal } \\
\text { Content Acquisition and } \\
\text { Al-2 Activity } \\
\text { Measurement } \\
\text { - Fecal sample } \\
\text { microbiota analysis } \\
\text { ELISA } \\
\text { RT-PCR-WB }\end{array}$ & $\begin{array}{l}\text { - The Al-2 level was significantly decreased in } \\
\text { the NEC group } \\
\text { - In the NA (NEC + Al-2) group; the intestinal } \\
\text { injury scores, expression of TLR4, NF-kB, and } \\
\text { proinflammatory factors were reduced, and } \\
\text { expression of anti-inflammatory factor was } \\
\text { increased compared to NEC group } \\
\text { - At the phylum level, the Proteobacteria } \\
\text { abundance in the NA group was significantly } \\
\text { increased. } \\
\text { - At the genus level, Helicobacter and } \\
\text { Clostridium exhibited significantly greater } \\
\text { abundance in the NEC group compared to the } \\
\text { other two groups }\end{array}$ & Ji et al. \\
\hline Review & Drosophila & $\begin{array}{l}\text { Different } \\
\text { pathogens }\end{array}$ & $\begin{array}{l}\text { Gaining proper insight into } \\
\text { host-pathogen interactions } \\
\text { using drosophila as a model }\end{array}$ & - Not applicable & $\begin{array}{l}\text { - In vivo drosophila studies enabled the } \\
\text { identification of humoral and cell-mediated host } \\
\text { defense factors against a wide array of } \\
\text { intracellular and extracellular pathogens }\end{array}$ & Younes et al. \\
\hline
\end{tabular}


macrophage depletion led to rapid embryo death. Interestingly, they reported that the depletion of cystic fibrosis transmembrane conductance regulator (CFTR) in zebrafish by morpholino significantly increased the mortality rate, bacterial burden, and abscess formation. This is consistent with previous studies showing that CFTR expression on both innate and adaptive immune cells contributes to immunological dysfunction in cystic fibrosis (CF) (Hu et al.; Johansen and Kremer; Li et al.; SermetGaudelus et al., 2003; Bruscia et al., 2009; Bonfield et al., 2012; Richards and Olivier, 2019).

Insect models proved to be very valuable for infectious diseases studies. Ménard et al., developed a non-mammalian infection model using larval G. mellonella to investigate the function of small regulatory RNAs (sRNAs) during $S$. aureus infection. The authors collected total RNA from $S$. aureus at several time points during the infection. They concluded that the expression patterns of the examined sRNAs were distinct and fluctuated over time, with small pathogenicity island RNA D $(s p r D)$ and small pathogenicity island RNA C ( $\operatorname{sprC})$ expression increasing during infection and correlated with larval death. In addition, they found out that when either $s p r D$ or $s p r C$ is deleted, the decline in insect death rates is delayed. The findings of Ménard et al. shed lights on the usefulness of G. mellonella as an infection model to investigate all 50 bona fide sRNAs known to be expressed by $S$. aureus.

Zhang et al., also utilized insects as a model to study the function of CLIPB10 in protease cascades "in vivo". Activation of prophenoxidase (proPO), a form of immune system regulation, leads to the formation of eumelanin on foreign microorganisms. ProPO activation is tightly controlled by different mechanisms including clip domain serine proteases (CLIPs), proteolytically inactive homologs, and serpin inhibitors. The authors studied the function of the CLIPB10 in Anopheles gambiae, which is the most common malaria vector in Sub-Saharan Africa. They reported that CLIPB10 was required for the melanization of ookinete stages of Plasmodium berghei. In addition, recombinant serpin 2 protein, a key inhibitor of the proPO activation cascade, efficiently inhibited CLIPB10 activity in vitro. The authors concluded that CLIPB10 along with CLIPB9 plays a critical role as the second protease with prophenoloxidase-activating function in A. gambiae suggesting functional redundancy in the protease network that controls melanization.

\section{REFERENCES}

Bonfield, T. L., Hodges, C. A., Cotton, C. U., and Drumm, M. L. (2012). Absence of the Cystic Fibrosis Transmembrane Regulator (Cftr) From Myeloid-Derived Cells Slows Resolution of Inflammation and Infection. J. Leukoc. Biol. 92, 1111-1122. doi: 10.1189/jlb.0412188

Bruscia, E. M., Zhang, P. X., Ferreira, E., Caputo, C., Emerson, J. W., Tuck, D., et al. (2009). Macrophages Directly Contribute to the Exaggerated Inflammatory Response in Cystic Fibrosis Transmembrane Conductance Regulator-/- Mice. Am. J. Respir. Cell Mol. Biol. 40, 295-304. doi: 10.1165/ rcmb.2008-01700C

Richards, C. J., and Olivier, K. N. (2019). Nontuberculous Mycobacteria in Cystic Fibrosis. Semin. Respir. Crit. Care Med. 40, 737-750. doi: 10.1055/s-0039-1693706

Sermet-Gaudelus, I., Le Bourgeois, M., Pierre-Audigier, C., Offredo, C., Guillemot, D., Halley, S., et al. (2003). Mycobacterium Abscessus and
Studying enterococcal pathogenesis generally requires vertebrates' models, making them slow, expensive, and ethically problematic (Weiner et al., 2016). Therefore, Revtovich et al., developed the first high-throughput Caenorhabditis elegans infection model. They successfully showed that E. faecium could effectively invade and colonize the intestine of $C$. elegans and elicit an immunological response. They used this model to examine a panel of lab strains with targeted virulence factor deletions. Although deletion of a single virulent factor such as $\Delta f m s 15$ was sufficient to affect virulence, multiple deletions were generally necessary to alter the pathogenesis, implying that host-pathogen interactions are multifactorial.

Autoinducer-2 (AI-2) is thought to be a bacterial interspecies signaling molecule that plays an important role in the physiological behaviors of bacteria. Ji et al., studied the role of AI-2 in Necrotizing enterocolitis (NEC) mouse model. They randomly divided $\mathrm{C} 57 \mathrm{BL} / 6$ mice into three groups: control, NEC, and NEC+AI-2 (NA). They reported that AI-2 significantly decreased in the NEC group. In addition, the intestinal injury scores, expression of TLR4, NF-kB, and proinflammatory factors were reduced in the NA group, whereas the expression of anti-inflammatory factors was increased in the NA group. They concluded that AI-2 partially reverses flora disorder and decreases inflammation in an NEC mouse model.

Younes et al., review discussed the use of drosophila in hostpathogen interaction research. Drosophila has recently gained a lot of attention due to the evolutionarily conserved features with higher vertebrates, such as cascades of the innate immune, pathways of signal transduction, and transcriptional regulators. The versatility, low cost, affordable maintenance, short life cycle, high fecundity, well-characterized genome made drosophila one of the most powerful model species (Younes et al.).

\section{AUTHOR CONTRIBUTIONS}

NY: drafted the editorial. GN: designed the work, revised the editorial critically for important intellectual content and provided final approval of the version to be published. All authors contributed to the article and approved the submitted version.

Children With Cystic Fibrosis. Emerg. Infect. Dis. 9, 1587-1591. doi: 10.3201/eid0912.020774

Weiner, L. M., Webb, A. K., Limbago, B., Dudeck, M. A., Patel, J., Kallen, A. J., et al. (2016). Antimicrobial-Resistant Pathogens Associated With HealthcareAssociated Infections: Summary of Data Reported to the National Healthcare Safety Network at the Centers for Disease Control and Prevention, 2011-2014. Infect. Control Hosp Epidemiol. 37, 1288-1301. doi: 10.1017/ice.2016.174

Conflict of Interest: The authors declare that the research was conducted in the absence of any commercial or financial relationships that could be construed as a potential conflict of interest.

Publisher's Note: All claims expressed in this article are solely those of the authors and do not necessarily represent those of their affiliated organizations, or those of the publisher, the editors and the reviewers. Any product that may be evaluated in 
this article, or claim that may be made by its manufacturer, is not guaranteed or endorsed by the publisher.

Copyright $\odot 2021$ Younes and Nasrallah. This is an open-access article distributed under the terms of the Creative Commons Attribution License (CC BY). The use, distribution or reproduction in other forums is permitted, provided the original author(s) and the copyright owner(s) are credited and that the original publication in this journal is cited, in accordance with accepted academic practice. No use, distribution or reproduction is permitted which does not comply with these terms. 


\section{OPEN ACCESS}

Edited by:

Gheyath Khaled Nasrallah, Qatar University, Qatar

Reviewed by:

Maria Kalamvoki,

University of Kansas Medical Center

United States

Peter Halfmann,

University of Wisconsin-Madison

United States

*Correspondence:

Ying Wu

wuy@sicau.edu.cn

Anchun Cheng

chenganchun@vip.163.com

†These authors have contributed equally to this work

Specialty section:

This article was submitted to Virus and Host,

a section of the journal

Frontiers in Cellular and Infection Microbiology

Received: 08 October 2019 Accepted: 16 December 2019

Published: 17 January 2020

Citation:

Li Y, Wu Y, Wang M, Ma Y, Jia R, Chen S, Zhu D, Liu M, Yang $Q$,

Zhao $X$, Zhang S, Huang J, Ou $X$, Mao S, Zhang L, Liu Y, Yu Y, Pan L,

Tian B, Rehman MU, Chen $X$ and Cheng A (2020) Duplicate US1 Genes

of Duck Enteritis Virus Encode a Non-essential Immediate Early Protein Localized to the Nucleus.

Front. Cell. Infect. Microbiol. 9:463.

doi: 10.3389/fcimb.2019.00463

\section{Duplicate US1 Genes of Duck Enteritis Virus Encode a Non-essential Immediate Early Protein Localized to the Nucleus}

\author{
Yangguang $\mathrm{Li}^{1,2,3+}$, Ying $\mathrm{Wu}^{1,2,3 * \dagger}$, Mingshu Wang ${ }^{1,2,3+}$, YunChao Ma ${ }^{1,2,3}$, Renyong Jia ${ }^{1,2,3}$, \\ Shun Chen ${ }^{1,2,3}$, Dekang Zhu ${ }^{2,3}$, Mafeng Liu ${ }^{1,2,3}$, Qiao Yang ${ }^{1,2,3}$, Xinxin Zhao ${ }^{1,2,3}$, \\ Shaqiu Zhang ${ }^{1,2,3}$, Juan Huang ${ }^{1,2,3}$, Xumin Ou ${ }^{1,2,3}$, Sai Mao ${ }^{1,2,3}$, Ling Zhang ${ }^{1,2,3}$, \\ Yunya Liu ${ }^{1,2,3}$, Yanling Yu ${ }^{1,2,3}$, Leichang Pan ${ }^{1,3}$, Bin Tian ${ }^{1,3}$, Mujeeb Ur Rehman ${ }^{1,3}$, \\ Xiaoyue Chen ${ }^{2,3}$ and Anchun Cheng ${ }^{1,2,3 *}$ \\ ${ }^{1}$ Institute of Preventive Veterinary Medicine, Sichuan Agricultural University, Wenjiang, China, ${ }^{2}$ Avian Disease Research \\ Center, College of Veterinary Medicine of Sichuan Agricultural University, Wenjiang, China, ${ }^{3}$ Key Laboratory of Animal \\ Disease and Human Health of Sichuan Province, Sichuan Agricultural University, Wenjiang, China
}

The duplicate US1 genes of duck enteritis virus (DEV) encode a protein with a conserved Herpes_IE68 domain, which was found to be closely related to the herpes virus immediate early regulatory protein family and is highly conserved among counterparts encoded by Herpes_IE68 genes. Previous studies found the homologous proteins HSV-1 ICP22 and VZV ORF63/ORF70 to be critical for virus transcription and replication. However, little is known about the DEV ICP22 protein. In this paper, we describe the characteristics of this protein based on pharmacological experiments, real-time quantitative Polymerase Chain Reaction, Western blot, and immunofluorescence assays. We also investigate the role of the protein in DEV replication via mutation of US1. As a result, we found that the DEV ICP22 protein is a non-essential immediate early protein predominantly located in the nucleus of infected DEF cells and that DEV replication is impaired by US1 deletion. We also found that ICP22 contains a classical nuclear localization signal (NLS) at 305-312AA, and ICP22 cannot enter the nucleus by itself after mutating residue 309.

Keywords: herpesvirus, DEV, US1, ICP22, IE gene, non-essential, NLS

\section{INTRODUCTION}

Duck plague is an acute, febrile, septic, and lethal infectious disease of ducks, geese, and swans (Cheng, 2015). The causative agent of this virus is known as duck plague virus (DPV) or duck enteritis virus (DEV), belonging to the alpha-herpesvirus superfamily of Herpesviridae (Bukreyev et al., 2014). Similar to its homologs, the DEV genome consists of a linear, double-stranded DNA comprising a unique long (UL), a unique short (US), a unique short internal repeat (IRS), and a unique short terminal repeat (TRS) region (UL-IRS-US-TRS) (Wu et al., 2012). Traditionally, viral genes have been believed to be expressed in a temporal cascade sequentially composed of immediate early (IE), early (E), and late (L) genes during lytic infection (Alfonsodunn et al., 2017). IE genes are transcribed immediately upon infection without the requirement of de novo protein synthesis, and early genes are commonly used to regulate viral replication. Late proteins form the capsid or surface receptors. 
Although some DEV genes have been studied in depth (MingSheng et al., 2008, 2010; Hua et al., 2009, 2011; Chanjuan et al., 2010; Wei et al., 2010; Wang et al., 2011; Wu et al., 2011; Zhang et al., 2011, 2017; He et al., 2012, 2018; Ying et al., 2012; Liu et al., 2016; Gao et al., 2017; Liu C. et al., 2017; Liu T. et al., 2017; Feng et al., 2018; Ma et al., 2018; You et al., 2018; Zhao et al., 2019), information regarding the DEV US1 gene is extremely limited. It is known that the DEV US1 gene is 990 bp in length and duplicated within the inverted repeat sequences delineating the US region of the genome (Ying et al., 2012). The homolog of its encoded protein ICP22 has been well described in Herpes simplex virus types 1 and 2 (HSV-1 and HSV-2) (Barcy and Corey, 2001; Lei et al., 2012; Zaborowska et al., 2014), Pseudorabies virus (PRV) (Cai et al., 2016), Equine herpes virus types 1 and 4 (EHV-1 and EHV-4) (Holden et al., 1995; Kim et al., 1997; Meulen et al., 2006), Bovine herpes virus type 1 (BHV-1) (Köppel et al., 1997), and Varicella zoster virus (VZV) (Di et al., 2005; Ambagala and Cohen, 2007). As one of the most important immediate early protein of HSV-1, ICP22 plays an important role in virus replication and transcriptional regulation and is necessary for acute replication of HSV-1 in eyes and neurons as well as the establishment of HSV-1 latent infection (Fraser and Rice, 2005; Rice and Davido, 2013).

Shortly after HSV-1 enters susceptible cells, the viral genome is transported to the nucleus, after which HSV-1 effectively recruits the RNA Pol II transcription machinery of host cells to transcribe viral genes at a high level while inhibiting the transcription of most host genes. The mechanism by which Pol II preferentially transcribes viral genes over host genes has not been determined, but some physical changes occur in Pol II itself (Fraser and Rice, 2005). According to previous work, ICP22 mediates two completely different effects on Pol II: induction of Pol IIi formation and loss of Pol II ser-2 phosphorylation (Ser-2P) (Zaborowska et al., 2016). It has also been shown that ICP22 promotes recruitment of the viral genome by transcription elongation factors, such as the FACT complex, to facilitate the transcriptional expression of the viral $\mathrm{L}$ gene in the late stage of infection (Fox et al., 2017). Furthermore, in the lytic infection phase of HSV-1 infection, the nucleocapsid assembled in the nucleus needs to enter the cytoplasm after initial packaging in the perinuclear space (Newcomb et al., 2017), with ICP22 having a regulatory role; that is, initial effective packaging of the newly produced nucleocapsid of HSV1 requires ICP22 (Yuhei et al., 2014). In addition, a novel function of ICP22 was recently identified, involving alteration of chaperone localization in host cells (Köppel et al., 1997). It can be seen from the above research that HSV-1 ICP22 regulates the transcriptional expression of certain viral genes to create a nuclear environment conducive to viral replication, thereby promoting effective virus replication in host cells. Therefore, ICP22 is of great significance to the life cycle of herpes virus in host cells as well as in the interaction between pathogens and host cells.

ORF63, the ICP22 homolog of VZV, which is critical for efficient establishment of latency (Ambagala and Cohen, 2007), does not affect RNAPII phosphorylation or host chaperones (Fraser and Rice, 2005). At the same time, other studies have reported that BICP22, the homolog of ICP22 in BHV-1, exerts a general repressive effect on each kinetic class (Köppel et al., 1997). This finding might indicate that ICP22 acts in a speciesor genus-specific manner. At present, the properties of the duplicate DEV US1 genes and their encoded proteins have not been determined, and additional research is warranted to determine whether DEV ICP22 acts in a manner similar to its homologs.

To describe the DEV US1 gene and its encoded protein, an ICP22-specific antibody was prepared, after which RT-qPCR, WB and IFA were used to analyse the transcription, expression level and intracellular localization of the duplicated US1 gene of DEV after infection or eukaryotic transfection. The US1 gene type was assessed using the nucleic acid synthesis inhibitor ACV and the protein synthesis inhibitor CHX (Liu et al., 2015). The results showed that DEV ICP22 is an immediate early protein located in the nucleus of DEF cells, which contains a classical NLS at 305-314 AA of the ICP22 protein. To elucidate the roles of DEV US1 and its NLS in virus replication, a two-step homologous recombination technique was used for US1 mutating, the results showed the US1 gene and its 308312AA is important for DEV replication. This is the first report of DEV US1 and these details regarding its role in virus replication will be helpful for further investigation of its function and mechanism.

\section{MATERIALS AND METHODS}

\section{Cells and Viruses}

Monolayer cultures of duck embryo fibroblasts (DEFs) were propagated in Dulbecco's Modified Eagle Medium (DMEM; Gibco, USA) supplemented with $10 \%$ newborn bovine serum (NBS) and incubated at $37^{\circ} \mathrm{C}$ in a $5 \% \mathrm{CO} 2$ humidified incubator. Wild-type DEV CHv (Ying et al., 2012) and its recombinant virus DEV BAC were generated in our laboratory (Ying et al., 2017).

\section{Plasmid Construction and Transfection}

All enzymes used for cloning processes were purchased from Takara (Takara, China), except for MonClone ${ }^{\mathrm{TM}}$ Hi-Fusion Cloning Mix V2 (Monad, China). ICP22 ORFs (composed of 990 bp) were amplified via PCR from the genomic DNA of DEV using primers P1 and P2 (Table 1) and purified using a PCR gel purification kit (Tiangen, China). Subsequently, the products were inserted into correspondingly digested pEGFP$\mathrm{N} 1$ and pet32a(+) vectors using EcoR I and Xho I QuickCut enzyme to create the recombinant plasmids EGFP-ICP22 and pet32a(+)-ICP22, and following the same steps for other plasmids. In this study, eukaryotic expression plasmids were transfected into $70-90 \%$ confluent cells using Lipofectamine ${ }^{\mathrm{TM}}$ 3000 Transfection Reagent (Invitrogen, USA) following the manufacturer's instructions.

\section{Generation of a Polyclonal Antibody Against the Recombinant ICP22 Protein}

As previously described Cheng et al. (2012), the plasmid pet32a(+)-ICP22 was transformed into Escherichia coli BL21 
TABLE 1 | Primers used in this study.

\begin{tabular}{|c|c|c|c|}
\hline Primer & Sequence $\left(5^{\prime}-3^{\prime}\right)$ & Purpose & $\begin{array}{l}\text { Length } \\
\text { (bp) }\end{array}$ \\
\hline P1F & $\begin{array}{l}\text { CTACCGGACTCAGATCTCGAG } \\
\text { ATGGCGACGGCA }\end{array}$ & & \\
\hline P1R & $\begin{array}{l}\text { GTACCGTCGACTGCAGAATT } \\
\text { CCACTCTTGGGGCGTIT } \\
\text { GTGGT }\end{array}$ & $\begin{array}{l}\text { Eukaryotic expression } \\
\text { of US1 }\end{array}$ & 990 \\
\hline P2F & $\begin{array}{l}\text { CCATGGCTGATATCGGAT } \\
\text { CCGAATTCTACAGAACTGCGA } \\
\text { TAGAC }\end{array}$ & $\begin{array}{l}\text { Prokaryotic expression } \\
\text { of US1 }\end{array}$ & 990 \\
\hline $\mathrm{P} 2 \mathrm{R}$ & $\begin{array}{l}\text { CAGTGGTGGTGGTGGTGGTG } \\
\text { CTCGAGACTCTTGGGGCGTIT } \\
\text { TGTGGT }\end{array}$ & & \\
\hline P3F & CGTAGCGTCACATCAAGCAG & ICP22 quantitative & 147 \\
\hline P3R & GCGTTTGGTCCCTATAACCTC & primers & \\
\hline P4F & TGGCATCCACGAAACTACC & $\beta$-Actin quantitative & 130 \\
\hline P4R & СTTCTGCATCCTGTCAGCGA & PCR primers & \\
\hline P5F & $\begin{array}{l}\text { CAATATATAAAAGGCTCTCGTT } \\
\text { TACAGAACTGCGATAGACAGG } \\
\text { ATGACGACGATAAGTAGGG }\end{array}$ & $\begin{array}{l}\text { Replacement of the } \\
\text { US1 gene by the kana- } \\
\text { resistance cassette }\end{array}$ & 1108 \\
\hline P5R & $\begin{array}{l}\text { AGGTTAATACGCGCTTGCAGC } \\
\text { ATATATCGCGACAGGTTAGTC } \\
\text { TATCGCAGTTCTGTAAACGAG } \\
\text { AGCCTITATATATTGTTAACC } \\
\text { AATTCTGATTAG }\end{array}$ & & \\
\hline P6F & $\begin{array}{l}\text { ATGGCGACGGCATCGCG } \\
\text { ACGGCCAAGCGGTCAAGCG } \\
\text { TGCGAGGATGACGACGATAA } \\
\text { GTAGGG }\end{array}$ & $\begin{array}{l}\text { Replacement of the } \\
\text { reverse US1 gene by } \\
\text { the reverse } \\
\text { kana-resistance }\end{array}$ & 1109 \\
\hline P6R & $\begin{array}{l}\text { TTAACTCTTGGGGCGTITIGTG } \\
\text { GTACCCGCGAGTGCGCTCAC } \\
\text { GCACGCTTGACCGCTTGGCC } \\
\text { GTCGCGATGCCGTCGCCATTA } \\
\text { ACCAATTCTGATAAG }\end{array}$ & & \\
\hline P7F & TCATTGCTCAATACGGGAAG & Identification of the & $1500 \& 5$ \\
\hline P7R & GCGGTGTITATTGACATCA & US1 gene deletion & \\
\hline P8F & GGACAGCGTACCACAGATAA & Amplification of the & 498 \\
\hline P8R & ACAAATCCCAAGCGTAG & UL30 fragment & \\
\hline P9F & ППтССтССTCСTCGCTGAGT & Probe primers & 60 \\
\hline P9R & GGCCGGGTTTGCAGAAGT & & \\
\hline P10 & $\begin{array}{l}\text { FAM-CCCTGGGTACAAGC } \\
\text { G-MGB }\end{array}$ & Probe & / \\
\hline P11F & $\begin{array}{l}\text { AAGTCCGGCCGGACTCAGATC } \\
\text { TCGAGCATGAGCGAAAAATAC } \\
\text { ATCG }\end{array}$ & $\begin{array}{l}\text { Prokaryotic expression } \\
\text { of GFPC2- } \beta \text {-Gal- } \\
\text { US1/ANLS/NLS }\end{array}$ & 3308 \\
\hline P11R & $\begin{array}{l}\text { GATGCCGTCGCCATACTGCA } \\
\text { GAATTCTIITGACACCAGA } \\
\text { CCAA }\end{array}$ & & \\
\hline P12R & $\begin{array}{l}\text { TTGGTCCCTATCATACTGC } \\
\text { AGAATCTIITGACACCAG } \\
\text { ACCAA }\end{array}$ & & \\
\hline P13F & $\begin{array}{l}\text { ATAGAGGTTATAGGGACCGCA } \\
\text { CGCAAACGGCCGACCACGAG } \\
\text { GAGTATG }\end{array}$ & $\begin{array}{l}\text { Prokaryotic expression } \\
\text { of GFPC2- } \beta \text {-Gal-US1 } \\
\text { amino acid mutation }\end{array}$ & 2200 \\
\hline P13R & $\begin{array}{l}\text { GTCGGCCGTTTGCGTGCGGT } \\
\text { CCCTATAACCTCTATAACT }\end{array}$ & & \\
\hline
\end{tabular}

(Continued)
TABLE 1 | Continued

\begin{tabular}{|c|c|c|c|}
\hline Primer & Sequence $\left(5^{\prime}-3^{\prime}\right)$ & Purpose & $\begin{array}{l}\text { Length } \\
\text { (bp) }\end{array}$ \\
\hline $\mathrm{P} 14 \mathrm{~F}$ & $\begin{array}{l}\text { AGGTTATAGGGACCAAAGCCA } \\
\text { AACGGCCGACCACGAGGAGT } \\
\text { ATG }\end{array}$ & & \\
\hline P14R & $\begin{array}{l}\text { CGTGGTCGGCCGTTIGGCTIT } \\
\text { GGTCCCTATAACCTCTAT }\end{array}$ & & \\
\hline P15F & $\begin{array}{l}\text { TTATAGGGACCAAACGCGCAC } \\
\text { GGCCGACCACGAGGAGTATG } \\
\text { AGC }\end{array}$ & & \\
\hline P15R & $\begin{array}{l}\text { TACTCCTCGTGGTCGGCCGTG } \\
\text { CGCGTTTGGTCCCTATAACCT }\end{array}$ & & \\
\hline P16F & $\begin{array}{l}\text { AGGGACCAAACGCAAAGCGC } \\
\text { CGACCACGAGGAGTATGAGC } \\
\text { GCA }\end{array}$ & & \\
\hline P16R & $\begin{array}{l}\text { ATACTCCTCGTGGTCGGCGCT } \\
\text { TTGCGTTGGTCCCTATAA }\end{array}$ & & \\
\hline P17F & $\begin{array}{l}\text { CAAACGCAAACGGGCGACC } \\
\text { ACGAGGAGTATGAGCG } \\
\text { CACTCG }\end{array}$ & & \\
\hline P17R & $\begin{array}{l}\text { TCATACTCCTCGTGGTCGCCC } \\
\text { GTTGCGTTGGTCCCTATAA }\end{array}$ & & \\
\hline P18F & $\begin{array}{l}\text { GCTACCATTACCAGTTGGTCT } \\
\text { GGTGTCAAAAAGAATTCTG }\end{array}$ & & \\
\hline P18R & $\begin{array}{l}\text { AAAACGATTCCGAAGCCCAAC } \\
\text { CTITCATAGAAGGCG }\end{array}$ & & \\
\hline P19F & $\begin{array}{l}\text { CGACTCTGAAAGCGAAGTTAT } \\
\text { AGAGGTTATAGGGACCAAAGC } \\
\text { CAGGATGACGACGATAAGTAG } \\
\text { GG }\end{array}$ & $\begin{array}{l}\text { Replacement of the } \\
\text { US1 309aa by the } \\
\text { kana-resistance } \\
\text { cassette }\end{array}$ & 1035 \\
\hline P19R & $\begin{array}{l}\text { TACCCGCGAGTGCGCTCATAC } \\
\text { TCCTCGTGGTCGGCCGTTIGG } \\
\text { CTITGGTCCCTATAACCTCTAT } \\
\text { AACTTCGCTTCAGAGTCGTTA } \\
\text { ACCAATTCTGATTAG }\end{array}$ & & \\
\hline P2OF & $\begin{array}{l}\text { CTCCGACTCTGAAAGCGAAGT } \\
\text { TATAGAGGTTATAGGGACCGC } \\
\text { AGCCGCAGCGGCGACCACGA } \\
\text { GGAGTATGAGCGCAGGATGA } \\
\text { CGACGATAAGTAGGG }\end{array}$ & $\begin{array}{l}\text { Replacement of the } \\
\text { US1 308-312aa by the } \\
\text { kana-resistance } \\
\text { cassette }\end{array}$ & 1035 \\
\hline P20R & $\begin{array}{l}\text { GTITGTGGTACCCGCGAGTG } \\
\text { CGCTCATACTCCTCGTGGTCG } \\
\text { CCGCTGCGGCTGCGGTCCCT } \\
\text { ATAACCTCTATAATTAACCAAT } \\
\text { TCTGATTAG }\end{array}$ & & \\
\hline
\end{tabular}

for protein expression. Bacterial pellets were resuspended in 20 $\mathrm{mmol} / \mathrm{L}$ Tris-HCL buffer, and the supernatant was incubated with Ni-NTA his• Bind ${ }^{\circledR}$ Resin (Millipore, USA) at low temperature for $2 \mathrm{~h}$. The samples were centrifuged to obtain a precipitate that was washed three times with washing solution to remove non-target proteins. The ICP22 protein was further purified by $10 \%$ SDS-PAGE gel and electroelution and used to generate a polyclonal antibody in mice.

\section{Real-Time Quantitative PCR (RT-qPCR)}

RT-qPCR was performed primarily according to a previous method (He et al., 2018). Briefly, DEF cells were infected with 
DEV at an MOI of 10 . Total RNA was isolated from the DEVinfected DEF cells at different time points post-infection (0.5, 2, 4, 8, 12, 24, 36, and $72 \mathrm{~h}$ ) using TRIzol (Invitrogen, USA). cDNA was synthesized in a reverse transcriptase reaction using a PrimeScript ${ }^{\mathrm{TM}} \mathrm{RT}$ reagent Kit with gDNA Eraser (Takara, China). Subsequently, qPCR was performed in a $10-\mu \mathrm{L}$ reaction volume. The primers P3 and P4 used are listed in Table 1. The $\beta$-actin gene was used as an endogenous control to normalize differences in the amount of total RNA in each sample. RT-qPCR was performed in triplicate and analyzed using the $2^{-\Delta \mathrm{CT}}$ threshold cycle method.

\section{Pharmacological Inhibition Reaction}

The procedure was performed as described previously (Liu et al., 2015). Briefly, the nucleic acid synthesis inhibitor ACV and the protein synthesis inhibitor CHX were used for determination of the US1 gene type. In brief, total RNA was isolated from DEV-infected DEF cells incubated with ACV or CHX at $24 \mathrm{~h}$ post-infection and reverse-transcribed into cDNA. The cDNA was used for PCR analysis, and the product was identified using agarose gel electrophoresis.

\section{Western Blotting Analysis}

As previously described Li et al. (2011), DEFs infected with DEV or treated with drugs were harvested at the indicated time points for sample preparation. The samples were separated by $10 \%$ SDS-PAGE and transferred to polyvinylidene fluoride (Millipore, USA) membranes. The membranes were blocked with 5\% BSA TBS buffer containing $0.05 \%$ Tween-20 for $2 \mathrm{~h}$ and incubated with the diluted primary antibody for $2 \mathrm{~h}$ at $37^{\circ} \mathrm{C}$. The membrane was washed with TBST and incubated with goat anti-mouse HRP-labeled IgG (Abcam, Britain) secondary antibodies for $1 \mathrm{~h}$ at $37^{\circ} \mathrm{C}$. After washing, the membrane was developed using an ECL kit.

\section{Immunofluorescence Assay}

IFA was performed as previously described Liu et al. (2015), with minor modifications. Briefly, $4 \%$ paraformaldehyde was used to fix DEF cells grown on coverslips in 6-well plates at different time points. The cells were permeabilized in $0.25 \%$ Triton X-100 and blocked in 5\% BSA at $37^{\circ} \mathrm{C}$. The anti-ICP22 antibody and FITC/TRITC-conjugated IgG (Invitrogen, USA) were used as primary and secondary antibodies, respectively. All antibodies were diluted in 1\% BSA PBS. The cells were treated with 4'6-diamidino-2-phenylindole (DAPI) for $15 \mathrm{~min}$ to stain the nucleus. Images were captured using a fluorescence microscope after the coverslips were sealed onto glass slides with glycerine buffer.

\section{Construction and Identification of the US1 Mutant Viruses}

The Red-mediated recombination scheme is shown in Figure 5A (consider the first US1 gene deletion as an example), primarily referring to the method of Tischer et al. (2006, 2010). Transfection of DEV in BAC- $\triangle$ US1-GS1783 and DEV in BAC-2 $\triangle$ US1-GS1783 plasmids into DEFs was subsequently performed; when fluorescence was observed, the cell medium was collected to obtain recombinant virus DEV CHv-BACG- $\triangle$ US1 (BAC- $\triangle$ US1) and DEV CHv-BAC-G-2 $\triangle$ US1 (BAC$2 \Delta \mathrm{US1}$ ) (Figure 5B). The identification of BAC- $\triangle \mathrm{US} 1$ and BAC$2 \Delta \mathrm{US} 1$ is depicted in Figure 6. As shown in Figure 6A, $1 \mu \mathrm{g}$ of plasmid was digested for $30 \mathrm{~min}$ with $\mathrm{BamH}$ I or Xho I at $37^{\circ} \mathrm{C}$, and then agarose gel electrophoresis was carried out at $30 \mathrm{~V}$ for $\sim 10$ h. We used P7 to identify recombinant viral genomic DNA to ensure that the recombinant virus was not contaminated by the parental strain BAC (Figure 6B). The construction process of DEV CHv-BAC-G- $\triangle$ US1-R309A (BAC- $\triangle$ US1-R309A) and DEV CHv-BAC-G- $\Delta$ US1-308-312AA (BAC- $\Delta$ US1-308-312AA) is the same as above.

\section{Growth Curve and Determination of DNA Copies}

Growth curve analysis was performed as described previously (Ma et al., 2018). Briefly, the growth kinetics of US1 mutant viruses were compared to that of the parental strain. Cell cultures were infected at an MOI of 0.01 (multi-step assay) or 2 (singlestep assay). After $2 \mathrm{~h}$ of adsorption, the cells were washed and then overlaid with DMEM containing $2 \%$ NBS. Supernatants and infected cells were separately harvested at $24,48,72,96 \mathrm{~h}$ (multistep assay) or $6,12,18,24,36 \mathrm{~h}$ (single-step assay) at successive intervals, and the amount of infectious virus was determined by plaque assay using DEF cells.

The viral dosage and sampling time points were the same as for the growth curve detection described above. First, the UL30 DNA fragment was amplified with primer P8, and the fragment diluted in a 10-fold gradient was used as a template followed by the addition of primer P9 and probe P10 for qPCR to construct a standard curve: $\mathrm{Y}=-4.262 \mathrm{X}+43.675$. Primers $\mathrm{P} 8,9$, and 10 used in this part are listed in Table 1. The viral DNA in the samples was extracted using a Viral RNA/DNA Extraction Kit (Takara, China). qPCR was performed after the addition of primer P9 and probe P10, and viral DNA copies were estimated at various time points using the standard curve. All of the analyses were performed independently in triplicate with the standard error.

\section{RESULTS}

\section{Generation of a Polyclonal Antibody Against the Recombinant ICP22 Protein}

Polyclonal antiserum was generated using purified protein ICP22 to evaluate changes in intracellular localization and protein expression of DEV US1. As shown in Figure 1A, a distinct band with a molecular mass of $\sim 75 \mathrm{kDa}$ was visible when pet32a $(+)$ ICP22 expression was induced using IPTG in E. coli BL21 at $37^{\circ} \mathrm{C}$ for $6 \mathrm{~h}$; the ICP22 protein molecular mass is $\sim 35 \mathrm{kDa}$, that of the His-tagged protein is approximately $17 \mathrm{kDa}$, and pet32a $(+)$ is approximately $20 \mathrm{kDa}$. Next, the fusion protein was purified by gel electrophoresis and electroelution to generate the mouse anti-ICP22 protein polyclonal antibody. After a total of 5 weeks, polyclonal sera were collected, and the ELISA titer of the anti-US1 antibody was determined to be 1:32,000.

DEV was individually inoculated or transfected into DEFs with eukaryotic expression plasmid EGFP-ICP22 and tested 


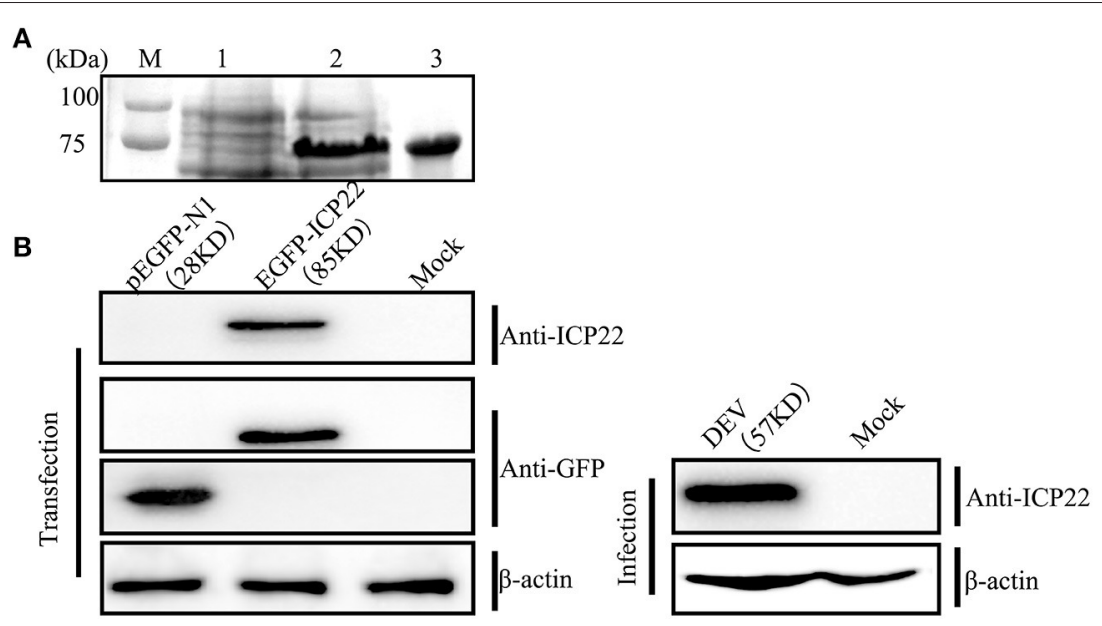

FIGURE 1 | Prokaryotic expression of DEV ICP22 and identification of the ICP22 protein. (A) M, Protein molecular mass marker; Lane 1, untreated samples; lane 2 , recombinant bacterial supernatant after IPTG induction; lane 3, the purified ICP22 protein. (B) Identification of the ICP22 protein by the prepared anti-ICP22 antibody in the eukaryotic expression plasmid EGFP-ICP22 transfection group (left panel) or DEV infection group (right panel).

against each other using the ICP22 antibody and a commercial GFP antibody to assess the specificity of the homemade antibody. As shown in Figure 1B, the EGFP-ICP22 fusion protein is $\sim 85$ $\mathrm{kDa}$ (left panel), whereas an $\sim 57-\mathrm{kDa}$ band was observed after DEV infection (right panel).

\section{Transcription Kinetics of the DEV US1 Gene}

The transcription kinetics of the DEV US1 gene were plotted to investigate variations in the transcriptional level of the DEV US1 gene in DEFs during infection. The results of RT-qPCR showed that the US1 gene began to be transcribed at $0.5 \mathrm{~h}$ after infection, with the amount of transcription increasing with time and peaking at $36 \mathrm{~h}$ after infection, similar to the transcriptional profile of the immediate early gene UL54 of DEV (Liu et al., 2015). After reaching the peak, the relative transcription level began to decrease gradually (Figure 2).

\section{Dynamic Expression of the DEV US1-Encoded Protein in DEFs}

To analyse the dynamic expression level of the ICP22 protein in infected cells more intuitively, samples were lysed at different times after viral infection for Western blot analysis. The US1encoded ICP22 protein could be detected at $2 \mathrm{~h}$ after virus infection, and its expression level was stable from 12 to 36 hpi, peaked at $24 \mathrm{hpi}$, and decreased significantly after $48 \mathrm{hpi}$. Expression of the endo-reference protein $\beta$-actin was stable throughout infection (Figure 3).

\section{Pharmacological Inhibition Test}

Drug inhibition experiments were performed to determine US1 gene types. As shown in Figure 4, DEV US1 was still detected after treatment with the nucleic acid synthesis inhibitor ACV or the protein synthesis inhibitor CHX, which was the same as that of the measured immediate early gene UL54 (Liu et al.,

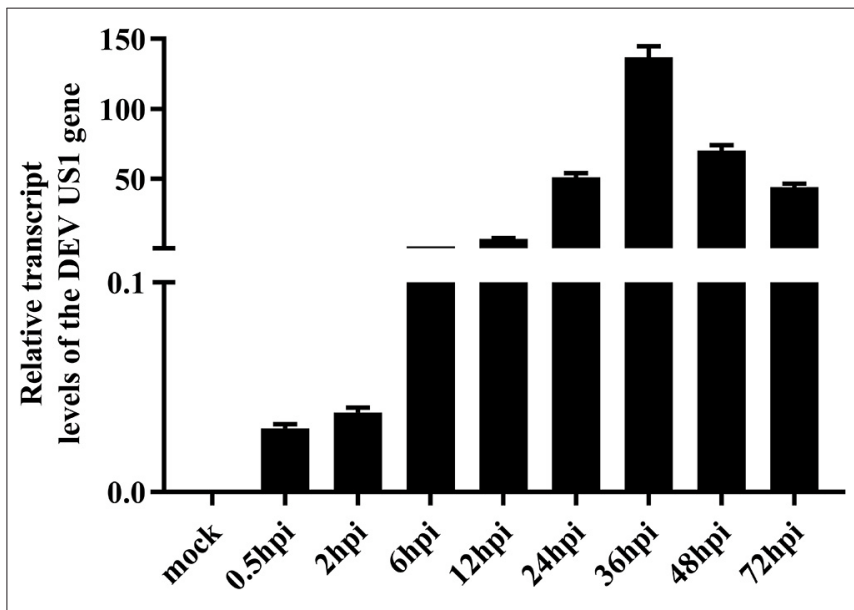

FIGURE 2 | Transcriptional analysis of the DEV US1 gene in infected cells with $10 \mathrm{MOI}$ DEV; the average relative content of the DEV US1 gene transcripts was calculated at the indicated time points using GraphPad Prism 8.0 software. Each time point was examined in triplicate with the standard error.

2015). The reported E gene UL13 (Hu et al., 2017), L gene US2 (Gao et al., 2015), and $\beta$-actin were also detected as controls. The positive and negative lanes represent uninfected and infected DEF cells without any treatment, respectively. The pharmacological inhibition test indicated that the DEV US1 gene is an immediate early gene.

\section{Construction and Identification of the Duplicated US1 Deletion Mutant}

The construction of recombinant DEV in BAC- $\triangle$ US1-GS1783 and DEV in BAC-2 $\triangle$ US1-GS1783 were carried out following the flow diagram displayed in Figure 5A. As shown in Figure 5B, plasmids from the positive clone were transfected into DEFs. 
After fluorescent spots appeared, the cell medium was collected, and the recombinant viruses DEV CHv-BAC- $\triangle \mathrm{US} 1$ and DEV $\mathrm{CHv}-\mathrm{BAC}-2 \Delta \mathrm{US} 1$ with deletion of the US1 gene were obtained.

Identification of recombinant virus DEV CHv-BAC- $\triangle \mathrm{US} 1$ (BAC- $\Delta$ US1) and DEV CHv-BAC-2 $\Delta$ US1 (BAC-2 $\Delta$ US1) were carried out by enzyme digestion, IFA and Western blotting. As shown in Figure 6A, the DEV CHv-BAC and BAC- $\triangle \mathrm{US} 1$, $2 \Delta$ US1 plasmids were digested by $B a m H$ I or Xho I into multiple fragments of different sizes, and the band differences are indicated by arrows. In IFA and Western blot analyses, no specific red fluorescence or specific band was observed for DEV$2 \Delta \mathrm{US} 1$, which indicated that the DEV US1 deletion (BAC- $\triangle \mathrm{US} 1$ and BAC-2 $\triangle$ US1) were successfully constructed (Figures 6C,D).

\section{Determination of Growth and DNA Copies of US1 Mutants}

To better characterize ICP22, we evaluated the role of US1 in DEV infection in vitro by one-step and multi-step growth assays with DEF cells (Figure 7A). The results showed that after
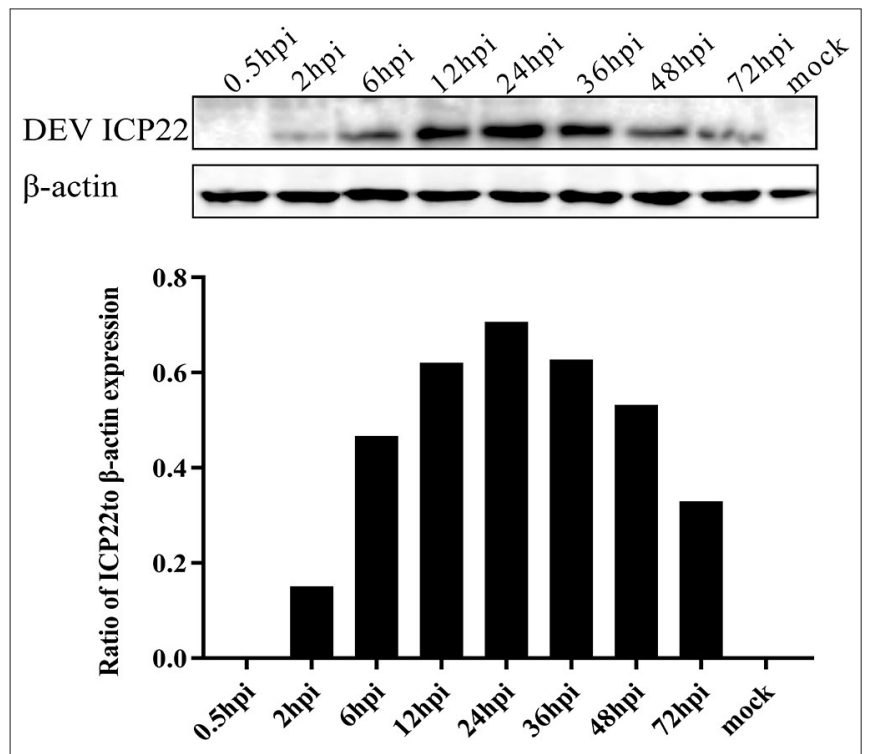

FIGURE 3 | Dynamic expression of ICP22 in DEV-infected cells. Expression of ICP22 in DEFs with $10 \mathrm{MOI}$ DEV was examined by Western blot assay (top panel). The quantitative result of WB is shown in the bottom panel.

Mock-infected DEFs were used as a control. infection of three strains in DEF cells at the same multiplicity of infection, a similar proliferation pattern was observed, with severe replication defects after deletion of US1. The virus titer of the deletion strain was significantly lower than that of the parent strain; in particular, at $18 \mathrm{~h}(2 \mathrm{MOI})$ and $48 \mathrm{~h}(0.01$ MOI) after infection, $2 \Delta$ US1 was nearly 100 times lower than DEV BAC. To further analyse the role of the US1 gene in viral replication, we detected the number of viral copies in the samples. Compared with BAC, a notable decrease was observed for -BAC-2 $\Delta$ US1 (Figure 7B), coinciding with the results of the growth curves.

\section{Intracellular Localization of ICP22}

The intracellular distribution of ICP22 was confirmed through IFA using mouse anti-ICP22 serum and FITC-conjugated goat anti-mouse IgG. As shown in Figure 8A, IFA results indicated that ICP2 2 began to enter the nucleus at $6 \mathrm{~h}$ after DEV infection, and all ICP22 had entered the nucleus at $24 \mathrm{~h}$. To determine whether ICP22 enters the nucleus by itself or with other viral proteins, EGFP-ICP22 was transfected into DEFs for analysis of ICP22 distribution. We found that ICP22 began to enter the nucleus at $12 \mathrm{~h}$ after transfection, with all inside the nucleus at $36 \mathrm{~h}$ (Figure 8B).

\section{ICP22 Is Located in Nucleus Independent of NLS Motif in Infected DEFs}

A series of plasmids were constructed to investigate how does ICP22 get into the nucleus (Figure 9A). As shown in Figure 9B, C2- $\beta-$ Gal-ICP2 2 and C2- $\beta$-Gal-ICP22 NLS were localized to the nucleus, which was consistent with the positive control group C2-SV40 NLS- $\beta$-Gal. However, the NLS deleted plasmid C2- $\beta$-Gal-ICP22 $\Delta$ NLS was localized in the cytoplasm, which was consisting with that of $\mathrm{C} 2-\beta-\mathrm{Gal}$ (Chen et al., 2018). Further mutating of alkaline acid K/R in 308-314AA to alanine found the C2- $\beta-\mathrm{Gal}-\mathrm{ICP} 22 \mathrm{R} 309 \mathrm{~A}$ and $\mathrm{C} 2-\beta-\mathrm{Gal}-$ ICP22 308-314AA were localized in the cytoplasm consisting with $\mathrm{C} 2-\beta-\mathrm{Gal}$.

Based on the above results, point mutation viruses DEV CHV-BAC- $\triangle$ US1 R309A and 308-312AA were constructed using Red recombinant system. As shown in Figure 10A, although ICP22 began to enter the nucleus at different time points after different virus infection, it could be located in the nucleus eventually. To better characterize the effect of DEV ICP22 R309A and 308-312AA on virus titer, we carried out the

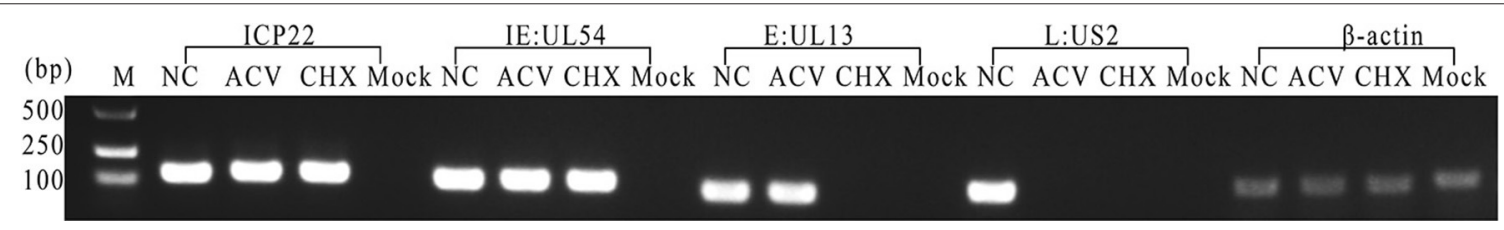

FIGURE 4 | Identification of the US1 gene type in DEV-infected cells. M, DL2000 Marker; ACV group, DEV-infected cells treated with $300 \mu \mathrm{g} / \mathrm{mL}$ nucleic acid synthesis inhibitor ACV; CHX group, DEV-infected cells treated with $50 \mu \mathrm{g} / \mathrm{mL}$ the protein synthesis inhibitor CHX; NC group, DEV-infected cells without drugs; Mock group, mock-infected DEFs were used as a control. 

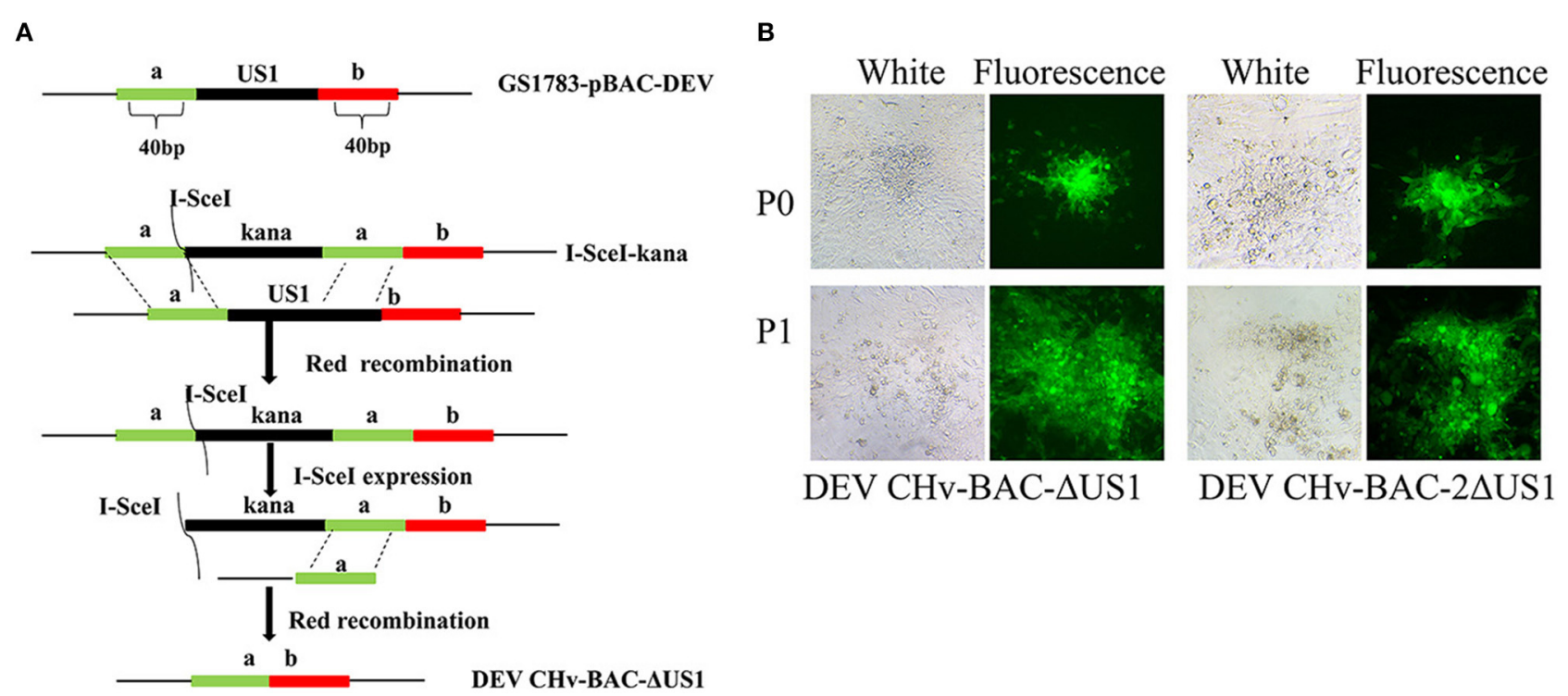

FIGURE 5 | Construction of DEV CHv-BAC- $\Delta$ US1 and DEV CHv-BAC-2 $\Delta$ US1. (A) Schematic diagram of constructing the duplicated US1 deletion mutant using the Red recombinant system. (B) DEV CHV-BAC- $\triangle U S 1$ and DEV CHV-BAC-2 $\Delta$ US1 were rescued in DEF cells.

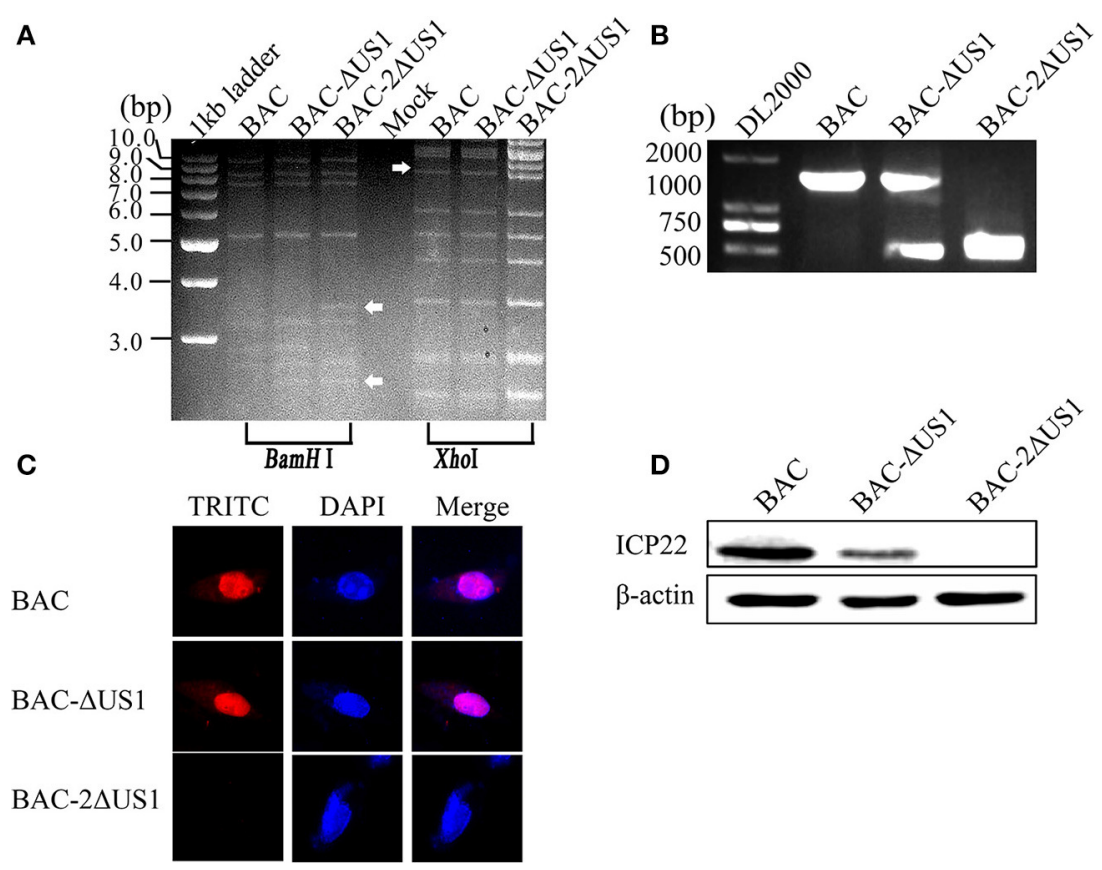

FIGURE 6 | Identification of BAC- $\triangle$ US1 and BAC-2 $\Delta$ US1. (A) Identification of BAC- $\Delta U S 1$ and BAC-2 $\Delta U S 1$ by enzyme digestion. M, 1 kb ladder marker; lane 4 , negative control. (B) Identification of DEV- $\Delta U S 1$ and DEV-2 $\Delta U S 1$ by PCR. (C) Identification of BAC- $\Delta U S 1$ and BAC-2 $\Delta U S 1$ by IFA. (D) Identification of BAC- $\Delta U S 1$ and BAC-2 $\triangle \mathrm{US} 1$ by Western blot analysis.

one-step and multi-step growth assays in vitro (Figure 10B). The result showed differential performance: the virus titer of the 308-312AA mutant was significantly higher than parent strain BAC- $\triangle$ US1, even nearly 100 times higher than DEV
BAC- $\triangle \mathrm{US} 1$ at 48 and $72 \mathrm{~h}$ after infection, but there is no obvious significant difference between the proliferation pattern between BAC-R309A and BAC-AUS10 (0.01MOI) and one-step growth assays. 


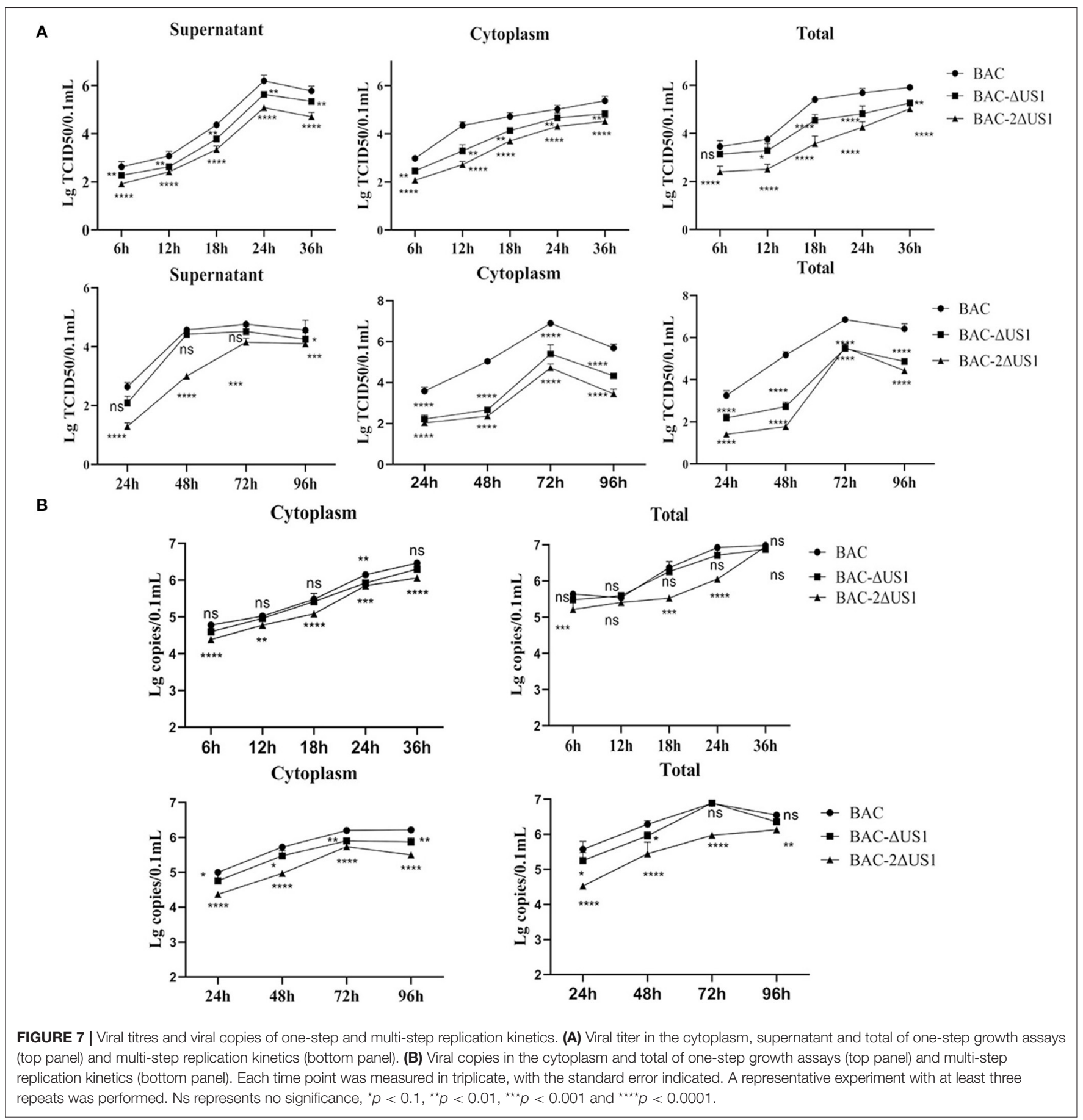

\section{DISCUSSION}

To prepare the anti-ICP22 polyclonal antibody, the recombinant expression plasmid pet32a(+)-ICP22 was constructed. To test the specificity of the homemade antibody, DEFs were transfected with EGFP-ICP22 or infection with DEV, demonstrating that the prepared ICP22 antibody can recognize ICP22 specifically in transfection or infection assays and that ICP22 expressed in DEFs is $\sim 57 \mathrm{kDa}$. Further commercial GFP antibody detection confirmed our findings. However, in our previous prokaryotic expression analysis, the ICP22 protein was found to be $\sim 35$ $\mathrm{kDa}$. These results suggest that some modifications occurred during eukaryotic expression, resulting in a migration of $\sim 22$ $\mathrm{kDa}$. It has been reported that modification of ICP22 is of great significance for its functions and occurs in other viruses; among them, different degrees of modification have been widely reported (Poon et al., 2000; O’Toole et al., 2003; Hoover et al., 2006; Bastian and Rice, 2009). According to 


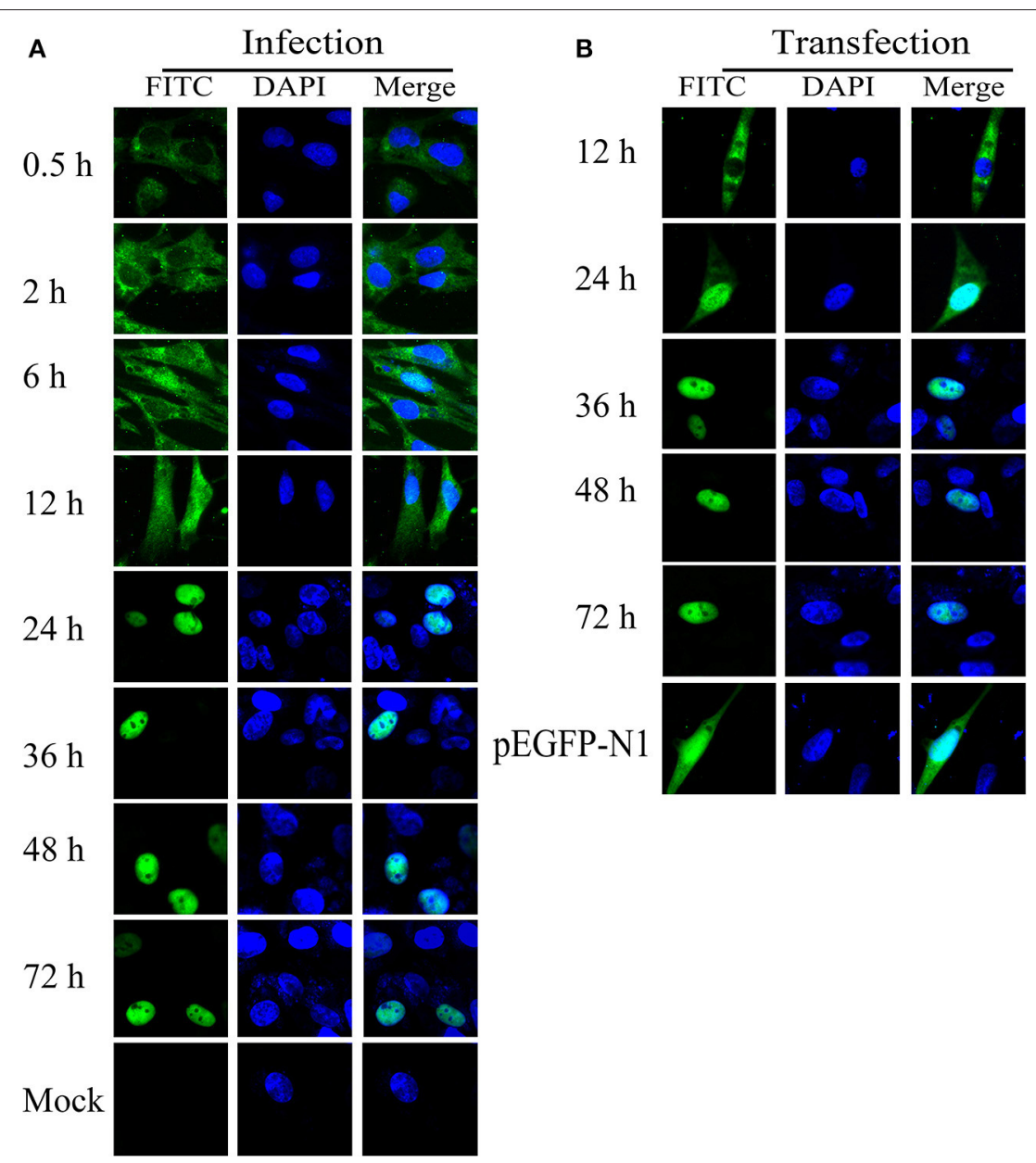

FIGURE 8 | Intracellular localization of ICP22 in DEFs. (A) Intracellular localization of ICP22 in infected DEFs at different time points with 10 MOI; mock was used as a control. (B) Intracellular localization of ICP22 at different time points in EGFP-ICP22-transfected DEFs. pEGFP-N1 was used as a control.

bioinformatics analysis (Figure S1), DEV ICP22 has multiple phosphorylation and glycosylation sites, which may be related to the observed migration pattern. Further research will focus on ICP22 modifications and effects on its function.

As mentioned earlier, lytic herpesvirus genes are believed to be expressed in a cascade sequentially composed of IE, E, and $\mathrm{L}$ genes during lytic infection (Alfonsodunn et al., 2017). We performed the following analyses to identify the US1 gene type. First, we investigated the relative expression of the DEV US1 gene in DEV-infected DEF cells at different time points using RT-qPCR. The results showed transcript expression as early as $0.5 \mathrm{~h}$ that gradually increased until peaking at $24 \mathrm{~h}$ (Figure 2). Subsequently, we detected the dynamic expression of ICP22 at the protein level at specific time points and transcript levels. The ICP2 2 protein was detected at $2 \mathrm{~h}$ after infection, and its expression level peaked at $24 \mathrm{~h}$, confirming the results of the translation studies and indicating that ICP22 can be expressed very early after infection with DEV. Finally, we identified the gene type of US1 using the nucleic acid synthesis inhibitor ACV and the protein synthesis inhibitor CHX (Liu et al., 2015). The pharmacological inhibition test showed that US1 was not affected by these two drugs. Combined with the above three tests, it can be determined that US1 is an immediate early gene, as is the reference gene UL54 (Liu et al., 2015).

US1 deletion mutants were also generated to investigate the roles of ICP22 in virus replication. The successful rescue of DEV CHv-BAC- $\triangle \mathrm{US} 1$ and DEV CHv-BAC-2 $\triangle \mathrm{US} 1$ recombinant strains indicated that the US1 gene is not required for DEV replication. The ensuing examination of the growth kinetics and viral DNA copies of US1 deletion mutants and parental virus showed a lower viral titer and DNA copy number for DEV-CHvBAC-2 $\Delta$ US1, indicating severe replication defects after deletion of the duplicate US1 genes, further suggesting that the US1 gene is important for DEV replication.

The intracellular localization of the protein is the basis for understanding the functions of a protein. In recent years, there have been reports that the ICP22 encoded by HSV-1 localizes to the cell nucleus independently of other viral proteins (Stelz et al., 2002). HSV-1 infection greatly alters the location of many host chaperones, including Hsc70, Hsp70, Hsp40, and Hsp90, 


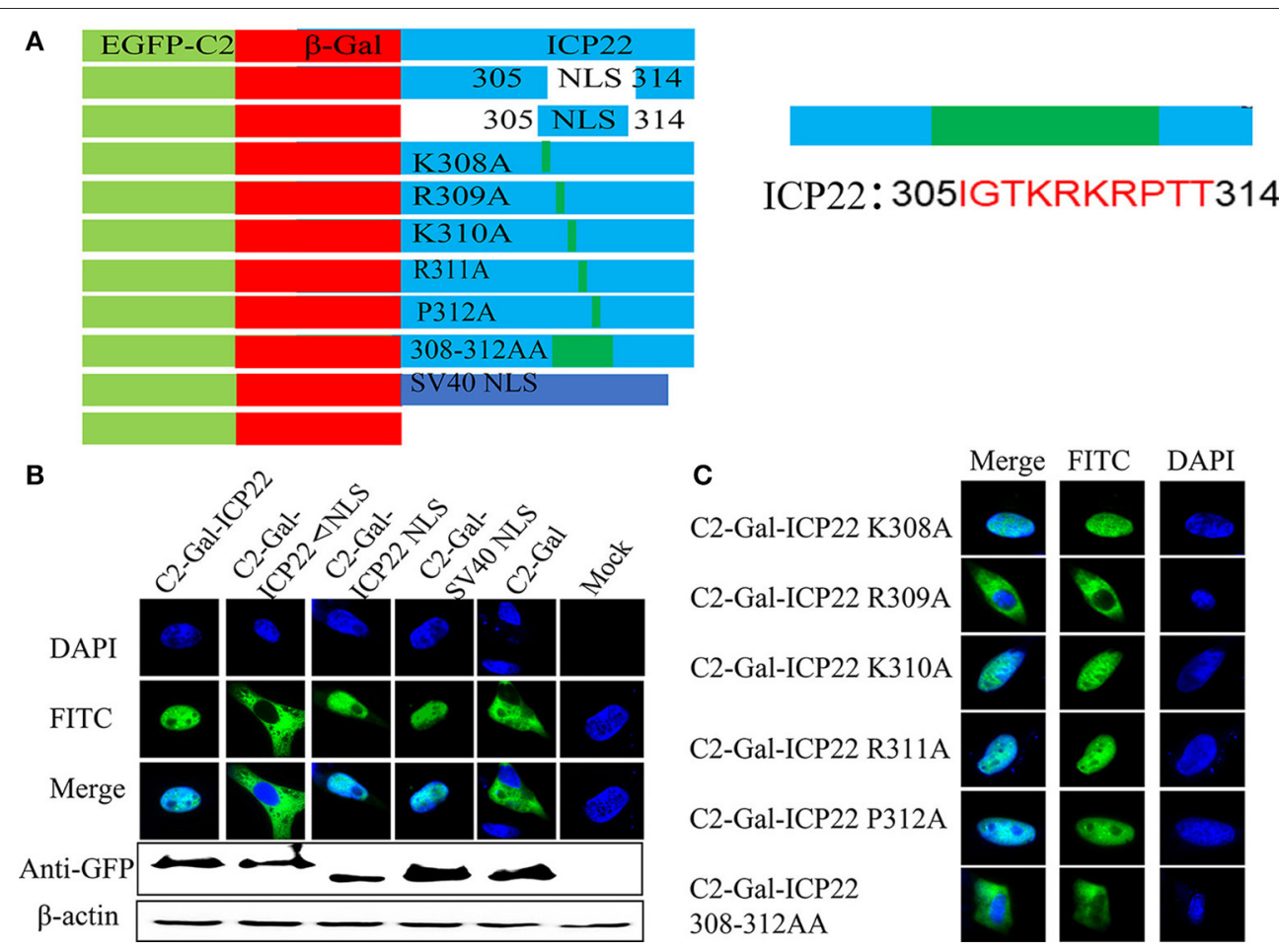

FIGURE 9 | Screening for key amino acids in NLS after transfection. (A) Schematic diagram of the ICP22 plasmids. (B) Localization of ICP22 in different plasmids transfected DEFs. mock was used as a control. (C) Localization of ICP22 in different mutation plasmids transfected DEFs.

causing their accumulation in the nucleosome, a region known as the virus-induced chaperone-enriched domain (VICE). Other studies have also reported that ICP22 localizes to VICE domains and is required for VICE domain formation during productive viral infection (Bastian et al., 2010). In our study, we found that ICP22 began to enter the nucleus at $6 \mathrm{~h}$ after DEV infection and that all of the protein had entered the nucleus at $24 \mathrm{~h}$ after DEV infection. To explore whether ICP22 localizes via active or passive transport, we constructed the ICP22 eukaryotic expression plasmid EGFP-ICP22 for transfection. As illustrated in Figure 1, the molecular weight of EGFP-ICP22 is $\sim 85 \mathrm{kDa}$, which is considerably $<50 \mathrm{kDa}$ (Macara, 2001; Sorokin et al., 2007). EGFP-ICP22 began to enter the nucleus at $12 \mathrm{~h}$ after transfection, and all had entered the nucleus at $36 \mathrm{~h}$, suggesting that the ICP22 protein has the ability to actively enter the nucleus. A series of plasmids and viruses were constructed to investigate how does ICP22 get into the nucleus. As shown in Figures 9, 10, we can conclude that the predicted NLS motif has strong nuclear import ability similar to that of SV40-NLS, and residue 309 is the key amino acid for determination of DEV ICP22 localization after transfection. But the NLS motif is not necessary for the localization of ICP22 in infected DEFs, and the delay of entering nucleus associated with DEV growth in vitro. According the previous studies, neither ICP4 nor ICP0 were recruited into NPDs (Newly synthesized Protein Domains, NPDs), while early in infection ICP22 was selectively recruited (Teo et al., 2016). And the ICP27 interacts with the C-terminal domain and is involved in the recruitment of RNAP II to viral transcription and replication compartments. During infection with ICP27 mutants that are unable to recruit RNAP II to viral replication sites, viral transcript levels were greatly reduced, viral replication compartments were poorly formed and Hsc70 focus formation was curtailed (Li et al., 2008). Combining the interaction between ICP22 and RNAP II, we speculate that ICP27 cooperates with the host protein to transport ICP22 to the nucleus. In addition, it is also possible that ICP22 contains other weak NLS. Therefore, it may be that other potential NLS and intracellular factors alone or together to drive ICP22 into the nucleus. However, this part of the experiment has not been carried out yet, in future, we will study the mechanism of ICP22 incorporation without NLS. In summary, we believe that the transformation of localization from the cytoplasm to the nucleus indicates that the position of synthesis is in the cytoplasm but that the function is exerted in the nucleus.

\section{CONCLUSIONS}

The DEV US1 ORF, as IE gene, is 990 bp in length and duplicated within the inverted repeat sequences delineating the US region of the genome. The molecular mass of the ICP22 protein is $\sim 57 \mathrm{kDa}$, and the protein can enter the nucleus by itself with a classical NLS motif. ICP22 cannot enter the nucleus by itself after mutating amino acid 309R, indicating the amino acid 309R is the key residue. Construction of the US1 mutant viruses and determination of the virus titer indicated that DEV US1 and its 


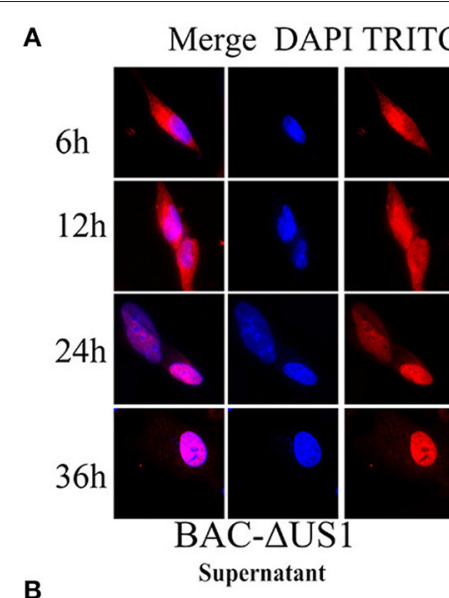

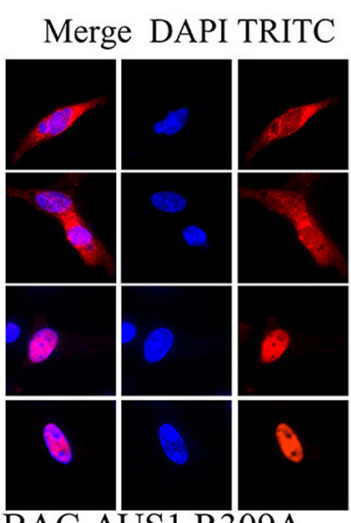

BAC- $\triangle$ US1 R309A Cytoplasm
Merge DAPI TRITC

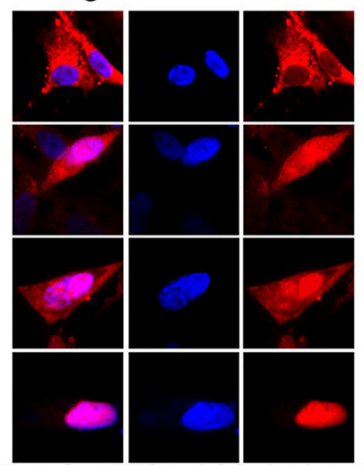

BAC- $\Delta$ US1 308-312AA

Total
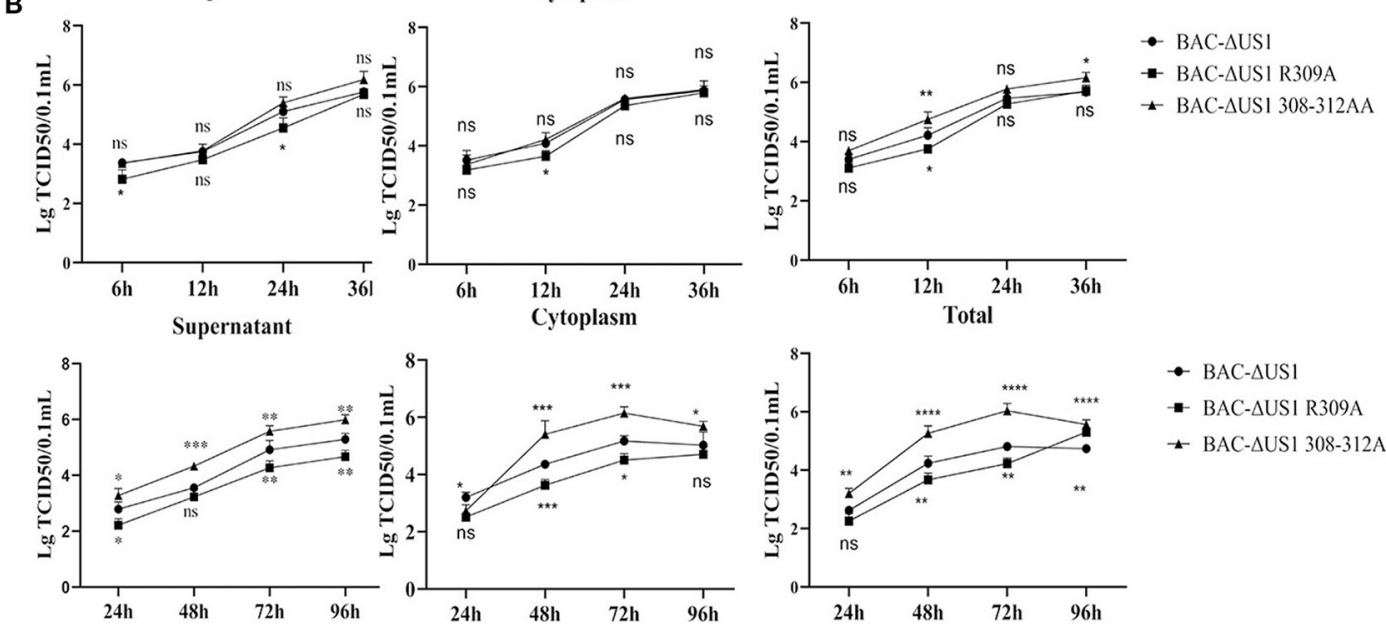

- BAC- $\triangle \mathrm{US}$

- BAC- $\triangle$ USI R309A

- BAC- $\triangle$ US1 308-312AA

FIGURE 10 | Intracellular localization of ICP22 and viral titres of one-step and multi-step replication kinetics. (A) Intracellular localization of ICP22 in infected DEFs at different time points with $10 \mathrm{MOI} B A C-\Delta U S 1$, BAC- $\Delta$ US1 R309A, and BAC- $\Delta$ US1 308-312AA. (B) Viral titer in the cytoplasm, supernatant, and total of one-step growth assays (top panel) and multi-step replication kinetics (bottom panel). Each time point was measured in triplicate, with the standard error indicated. A representative experiment with at least three repeats was performed. Ns represents no significance, ${ }^{\star} p<0.1,{ }^{\star \star} p<0.01,{ }^{\star \star \star} p<0.001$ and ${ }^{\star \star * \star} p<0.0001$.

NLS are non-essential but associated with a severe growth deficit in vitro.

\section{DATA AVAILABILITY STATEMENT}

All datasets generated for this study are included in the article/Supplementary Material.

\section{AUTHOR CONTRIBUTIONS}

YLi, YW, MW, and AC conceived and designed the experiments. MW, YM, RJ, SC, QY, DZ, ML, and XZ guided the experiment and helped analyse the data. SZ, JH, XO, SM, LZ, YLiu, YY, LP, $\mathrm{BT}, \mathrm{MR}$, and XC contributed materials. All authors read and approved the final manuscript.

\section{FUNDING}

This research was supported by the National Natural Science Foundation of China (Grant No. 31602079), a Key Project of the Education Department of Sichuan Province (Grant No. 17ZA0309), the China Agricultural Research System (CARS-42-17), Sichuan Province Research Programme (2017JY0014/2017HH0026) and the Integration and Demonstration of Key Technologies for Goose Industrial Chain in Sichuan Province (2018NZ0005).

\section{ACKNOWLEDGMENTS}

The authors thank YW for her review and revision and long-term spurs. We also thank AC and MW for their motivation in the experimental process of this paper.

\section{SUPPLEMENTARY MATERIAL}

The Supplementary Material for this article can be found online at: https://www.frontiersin.org/articles/10.3389/fcimb.2019. 00463/full\#Supplementary-material 


\section{REFERENCES}

Alfonsodunn, R., Turner, A. W., Jean Beltran, P. M., Arbuckle, J. H., Budayeva, H. G., Cristea, I. M., et al. (2017). Transcriptional elongation of HSV immediate early genes by the super elongation complex drives lytic infection and reactivation from latency. Cell Host Microbe 21, 507-517. doi: 10.1016/j.chom.2017.03.007

Ambagala, A. P., and Cohen, J. I. (2007). Varicella-Zoster virus IE63, a major viral latency protein, is required to inhibit the alpha interferon-induced antiviral response. J. Virol. 81, 7844-7851. doi: 10.1128/JVI.00325-07

Barcy, S., and Corey, L. (2001). Herpes simplex inhibits the capacity of lymphoblastoid B Cell lines to stimulate CD4+ T Cells. J. Immunol. 166, 6242-9. doi: 10.4049/jimmunol.166.10.6242

Bastian, T. W., Livingston, C. M., Weller, S. K., and Rice, S. A. (2010). Herpes simplex virus type 1 immediate-early protein ICP22 is required for VICE domain formation during productive viral infection. J. Virol. 84, 2384-2394. doi: 10.1128/JVI.01686-09

Bastian, T. W., and Rice, S. A. (2009). Identification of sequences in herpes simplex virus type 1 ICP22 that influence RNA polymerase II modification and viral late gene expression. J. Virol. 83, 128-139. doi: 10.1128/JVI.0 1954-08

Bukreyev, A. A., Chandran, K., Dolnik, O., Dye, J. M., Ebihara, H., Leroy, E. M., et al. (2014). Discussions and decisions of the 2012-2014 international committee on taxonomy of viruses (ICTV) Filoviridae study group, January 2012-June 2013. Arch. Virol. 159, 821-830. doi: 10.1007/s00705-013-1846-9

Cai, M., Si, J., Zeng, Z., Li, X., Mo, C., Yang, Y., et al. (2016). Probing the nuclear import signal and nuclear transport molecular determinants of PRV ICP22. Cell Biosci. 6, 1-10. doi: 10.1186/s13578-016-0069-7

Chanjuan, S., Anchun, C., Mingshu, W., Chao, X., Renyong, J., Xiaoyue, C., et al. (2010). Expression and distribution of the duck enteritis virus UL51 protein in experimentally infected ducks. Avian Dis. 54, 939-947. doi: 10.1637/9172-112109-ResNote.1

Chen, S., Liu, P., He, Y., Yang, C., Wang, M., Jia, R., et al. (2018). The $164 \mathrm{~K}, 165 \mathrm{~K}$ and $167 \mathrm{~K}$ residues in 160YPVVKKPKLTEE171 are required for the nuclear import of goose parvovirus VP1. Virology 519, 17-22. doi: 10.1016/j.virol.2018.03.020

Cheng, A. (2015). Duck Plague. Beijing: China agriculture press.

Cheng, A., Zhang, S., Zhang, X., Wang, M., Zhu, D., Jia, R., et al. (2012). Prokaryotic expression and characteristics of duck enteritis virus UL29 gene. Acta Virol. 56, 293-304. doi: 10.4149/av_2012_04_293

Di, E. V., Bontems, S., Habran, L., Jolois, O., Markinegoriaynoff, N., Vanderplasschen, A., et al. (2005). Varicella-zoster virus IE63 protein represses the basal transcription machinery by disorganizing the pre-initiation complex. Biol. Chem. 386, 255-267. doi: 10.1515/BC.2005.031

Feng, D., Cui, M., Jia, R., Liu, S., and Cheng, A. (2018). Co-localization of and interaction between duck enteritis virus glycoprotein $\mathrm{H}$ and L. BMC Vet. Res. 14:255. doi: 10.1186/s12917-018-1553-6

Fox, H. L., Dembowski, J. A., and Deluca, N. A. (2017). A herpesviral immediate early protein promotes transcription elongation of viral transcripts. MBio 8:e0745-17. doi: 10.1128/mBio.00745-17

Fraser, K. A., and Rice, S. A. (2005). Herpes simplex virus type 1 infection leads to loss of serine-2 phosphorylation on the carboxylterminal domain of RNA polymerase II. J. Virol. 79, 11323-11334. doi: 10.1128/JVI.79.17.11323-11334.2005

Gao, J., Cheng, A. C., Wang, M. S., Jia, R. Y., Zhu, D. K., Chen, S., et al. (2015). Identification and characterization of the duck enteritis virus (DEV) US2 gene. Genet. Mol. Res. 14:13779-90. doi: 10.4238/2015.October.28.40

Gao, X., Jia, R., Wang, M., Yang, Q., Chen, S., Liu, M., et al. (2017). Duck enteritis virus (DEV) UL54 protein, a novel partner, interacts with DEV UL24 protein. Virol. J. 14:166. doi: 10.1186/s12985-0170830-5

He, Q., Cheng, A., Wang, M., Xiang, J., Zhu, D., Zhou, Y., et al. (2012). Replication kinetics of duck enteritis virus UL16 gene in vitro. Virol. J. 9, 1-4. doi: 10.1186/1743-422X-9-281

He, T., Wang, M., Cao, X., Cheng, A., Ying, W., Qiao, Y., et al. (2018). Molecular characterization of duck enteritis virus UL41 protein. Virol. J. 15:12. doi: 10.1186/s12985-018-0928-4
Holden, V. R., Zhao, Y., Thompson, Y., Caughman, G. B., Smith, R. H., and O'Callaghan, D. J. (1995). Characterization of the regulatory function of the ICP22 protein of equine herpesvirus type 1. Virology 210: 273-82. doi: 10.1006/viro.1995.1344

Hoover, S. E., Cohrs, R. J., Rangel, Z. G., Gilden, D. H., Munson, P., and Cohen, J. I. (2006). Downregulation of varicella-zoster virus (VZV) immediateearly ORF62 transcription by VZV ORF63 correlates with virus replication in vitro and with latency. J. Virol. 80, 3459-3468. doi: 10.1128/JVI.80.7.34593468.2006

Hu, X., Wang, M., Chen, S., Jia, R., Zhu, D., Liu, M., et al. (2017). The duck enteritis virus early protein, UL13, found in both nucleus and cytoplasm, influences viral replication in cell culture. Poult. Sci. 96, 2899-2907. doi: 10.3382/ps/ pex043

Hua, C., Anchun, C., Mingshu, W., Jun, X., Wei, X., Fuxiao, S., et al. (2011). Expression and immunohistochemical distribution of duck plague virus glycoprotein gE in infected ducks. Avian Dis. 55, 97-102. doi: 10.1637/9487-072810-ResNote.1

Hua, C., Cheng, A., Wang, M., Guo, Y., Wei, X., and Lou, K. (2009). Complete nucleotide sequence of the duck plague virus gE gene. Arch. Virol. 154:163-5. doi: 10.1007/s00705-008-0284-6

Kim, S. K., Holden, V. R., and Callaghan, D. J. O. (1997). The ICP22 protein of equine herpesvirus 1 cooperates with the IE protein to regulate viral gene expression. J. Virol. 71, 1004-12.

Köppel, R., Vogt, B., and Schwyzer, M. (1997). Immediate-early protein BICP22 of bovine herpesvirus 1 trans-represses viral promoters of different kinetic classes and is itself regulated by BICP0 at transcriptional and posttranscriptional levels. Arch. Virol. 142, 2447-2464. doi: 10.1007/s007050050254

Lei, G., Wu, W. J., Liu, L. D., Wang, L. C., Ying, Z., Wu, L. Q., et al. (2012). Herpes simplex virus 1 ICP22 inhibits the transcription of viral gene promoters by binding to and blocking the recruitment of P-TEFb. PLOS ONE 7:e45749. doi: 10.1371/journal.pone.0045749

Li, L., Cheng, A., Wang, M., Xiang, J., Yang, X., Zhang, S., et al. (2011). Expression and characterization of duck enteritis virus gI gene. Virol. J. 8:241. doi: 10.1186/1743-422X-8-241

Li, L., Johnson, L. A., Dai-Ju, J. Q., and Sandri-Goldin, R. M. (2008). Hsc70 focus formation at the periphery of HSV-1 transcription sites requires ICP27. PLoS ONE 3:e1491. doi: 10.1371/journal.pone.0001491

Liu, C., Cheng, A., Wang, M., Chen, S., Jia, R., Zhu, D., et al. (2015). Duck enteritis virus UL54 is an IE protein primarily located in the nucleus. Virol. J. 12:198. doi: 10.1186/s12985-015-0424-Z

Liu, C., Cheng, A., Wang, M., Chen, S., Jia, R., Zhu, D., et al. (2016). Characterization of nucleocytoplasmic shuttling and intracellular localization signals in duck enteritis virus UL54. Biochimie 127, 86-94. doi: 10.1016/j.biochi.2016.05.003

Liu, C., Cheng, A., Wang, M., Chen, S., Jia, R., Zhu, D., et al. (2017). Regulation of viral gene expression by duck enteritis virus UL54. Sci. Rep. 7:1076. doi: 10.1038/s41598-017-01161-0

Liu, T., Cheng, A., Wang, M., Jia, R., Yang, Q., Wu, Y., et al. (2017). RNAseq comparative analysis of Peking ducks spleen gene expression $24 \mathrm{~h}$ postinfected with duck plague virulent or attenuated virus. Vet. Res. 48:47. doi: 10.1186/s13567-017-0456-Z

Ma, Y., Zeng, Q., Wang, M., Cheng, A., and Chen, X. (2018). US10 protein is crucial but not indispensable for duck enteritis virus infection in vitro. Sci. Rep. 8:16510. doi: 10.1038/s41598-018-34503-7

Macara, I. G. (2001). Transport into and out of the nucleus. Microbiol. Mol. Biol. Rev. 65, 570-594. doi: 10.1128/MMBR.65.4.570-594.2001

Meulen, K. V. D., Caij, B., Pensaert, M., and Nauwynck, H. (2006). Absence of viral envelope proteins in equine herpesvirus 1-infected blood mononuclear cells during cell-associated viremia. Vet. Microbiol. 113, 265-273. doi: 10.1016/j.vetmic.2005.11.048

Ming-Sheng, C., An-Chun, C., Ming-Shu, W., Li-Chan, Z., De-Kang, Z., Qi-Hui, L., et al. (2008). His6-tagged UL35 protein of duck plague virus: expression, purification, and production of polyclonal antibody. Intervirology 52, 141-151. doi: 10.1159/000221833

Ming-Sheng, C., An-Chun, C., Ming-Shu, W., Wan-Ping, C., Xian, Z., Shang$\mathrm{Xi}, \mathrm{Z}$., et al. (2010). Characterization of the duck plague virus UL35 gene. Intervirology 53, 408-416. doi: 10.1159/000317291 
Newcomb, W. W., Fontana, J., Winkler, D. C., Cheng, N., Heymann, J. B., and Steven, A. C. (2017). The primary enveloped virion of herpes simplex virus 1 : its role in nuclear egress. MBio 8:e00825-e00817. doi: 10.1128/mBio.00825-17

O’Toole, J. M., Aubert, M., Kotsakis, A., and Blaho, J. A. (2003). Mutation of the protein tyrosine kinase consensus site in the herpes simplex virus 1 $\alpha 22$ Gene Alters ICP22 posttranslational modification. Virology 305, 153-167. doi: 10.1006/viro.2002.1746

Poon, A. P., Ogle, W. O., and Roizman, B. (2000). Posttranslational processing of infected cell protein 22 mediated by viral protein kinases is sensitive to amino acid substitutions at distant sites and can be cell-type specific. J. Virol. 74, 11210-11214. doi: 10.1128/JVI.74.23.11210-11214.2000

Rice, S. A., and Davido, D. J. (2013). HSV-1 ICP22: hijacking host nuclear functions to enhance viral infection. Future Microbiol. 8, 311-321. doi: 10.2217/fmb.13.4

Sorokin, A. V., Kim, E. R., and Ovchinnikov, L. P. (2007). Nucleocytoplasmic transport of proteins. Biochemistry 72, 1439-1457. doi: 10.1134/S0006297907130032

Stelz, G., Rücker, E., Rosorius, O., Meyer, G., Stauber, R. H., Spatz, M., et al. (2002). Identification of two nuclear import signals in the $\alpha$-gene product ICP22 of herpes simplex virus 1. Virology 295, 360-370. doi: 10.1006/viro.2002.1384

Teo, C. S. H., Serwa, R. A., and O'Hare, P. (2016). Spatial and temporal resolution of global protein synthesis during HSV infection using bioorthogonal precursors and click chemistry. PLoS Pathog. 12:e1005927. doi: 10.1371/journal.ppat.1005927

Tischer, B. K., Smith, G. A., and Osterrieder, N. (2010). En passant mutagenesis: a two step markerless red recombination system. Methods Mol. Biol. 634, 421-430. doi: 10.1007/978-1-60761-652-8_30

Tischer, B. K., Von, E. J., Kaufer, B., and Osterrieder, N. (2006). Two-step red-mediated recombination for versatile high-efficiency markerless DNA manipulation in Escherichia coli. BioTechniques 40, 191-197. doi: 10.2144/000112096

Wang, M., Lin, D., Zhang, S., Zhu, D., Jia, R., Chen, X., et al. (2011). Prokaryotic expression of the truncated duck enteritis virus UL27 gene and characteristics of UL27 gene and its truncated product. Acta Virol. 56, 323-328. doi: 10.4149/av_2012_04_323

Wei, X., Anchun, C., Mingshu, W., Hua, C., Dekang, Z., and Qihui, L. (2010). Molecular cloning and characterization of the UL31 gene from duck enteritis virus. Mol. Biol. Rep. 37, 1495-1503. doi: 10.1007/s11033-009-9546-y

Wu, Y., Cheng, A., Wang, M., Zhang, S., Zhu, D., Jia, R., et al. (2011). Characterization of the duck enteritis virus UL55 protein. Virol. J. 8, 256-256. doi: 10.1186/1743-422X-8-256

Wu, Y., Cheng, A., Wang, M., Zhu, D., Jia, R., Chen, S., et al. (2012). Comparative genomic analysis of duck enteritis virus strains. J. Virol. 86, 13841-13842. doi: 10.1128/JVI.01517-12
Ying, W., Anchun, C., Mingshu, W., Qiao, Y., Dekang, Z., Renyong, J., et al. (2012). Complete genomic sequence of Chinese virulent duck enteritis virus. J. Virol. 86:5965. doi: 10.1128/JVI.00529-12

Ying, W., Li, Y., Wang, M., Sun, K., Jia, R., Chen, S., et al. (2017). Preliminary study of the UL55 gene based on infectious Chinese virulent duck enteritis virus bacterial artificial chromosome clone. Virol. J. 14:78. doi: 10.1186/s12985-017-0748-y

You, Y., Liu, T., Wang, M., Cheng, A., Jia, R., Yang, Q., et al. (2018). Author correction: duck plague virus glycoprotein $\mathrm{j}$ is functional but slightly impaired in viral replication and cell-to-cell spread. Sci. Rep. 8:6488. doi: 10.1038/s41598-018-24845-7

Yuhei, M., Keiko, S., Zhuoming, L., Masaaki, O., Hiroko, K. H., Jun, A., et al. (2014). Role of herpes simplex virus 1 immediate early protein ICP22 in viral nuclear egress. J. Virol. 88, 7445-7454. doi: 10.1128/JVI.01057-14

Zaborowska, J., Baumli, S., Laitem, C., O’Reilly, D., Thomas, P. H., O'Hare, P., et al. (2014). Herpes simplex virus 1 (HSV-1) ICP22 protein directly interacts with cyclin-dependent kinase (CDK) 9 to Inhibit RNA Polymerase II transcription elongation. PLOS ONE 9:e107654. doi: 10.1371/journal.pone. 0107654

Zaborowska, J., Isa, N. F., and Murphy, S. (2016). P-TEFb goes viral. Bioessays 1, S75-85. doi: 10.1002/bies.201670912

Zhang, D., Lai, M., Cheng, A., Wang, M., Wu, Y., Yang, Q., et al. (2017). Molecular characterization of the duck enteritis virus US10 protein. Virol. J. 14:183. doi: 10.1186/s12985-017-0841-2

Zhang, S., Xiang, J., Cheng, A., Wang, M., Wu, Y., Yang, X., et al. (2011). Characterization of duck enteritis virus UL53 gene and glycoprotein K. Virol. J. 8, 235-235. doi: 10.1186/1743-422X-8-235

Zhao, C., He, T., Xu, Y., Wang, M., and Zhang, L. (2019). Molecular characterization and antiapoptotic function analysis of the duck plague virus Us5 gene. Sci. Rep. 9:4851. doi: 10.1038/s41598-019-41311-0

Conflict of Interest: The authors declare that the research was conducted in the absence of any commercial or financial relationships that could be construed as a potential conflict of interest.

Copyright (c) $2020 \mathrm{Li}$, Wu, Wang, Ma, Jia, Chen, Zhu, Liu, Yang, Zhao, Zhang, Huang, Ou, Mao, Zhang, Liu, Yu, Pan, Tian, Rehman, Chen and Cheng. This is an open-access article distributed under the terms of the Creative Commons Attribution License (CC BY). The use, distribution or reproduction in other forums is permitted, provided the original author(s) and the copyright owner(s) are credited and that the original publication in this journal is cited, in accordance with accepted academic practice. No use, distribution or reproduction is permitted which does not comply with these terms. 
OPEN ACCESS

Edited by:

Layla Kamareddine,

Qatar University, Qatar

Reviewed by:

Nathalie Chazal,

Université de Montpellier, France

Miguel A. Martín-Acebes,

Instituto Nacional de Investigación y

Tecnología Agraria y Alimentaria

(INIA), Spain

${ }^{*}$ Correspondence:

Anchun Cheng

chenganchun@vip.163.com

Renyong Jia

jiary@sicau.edu.cn

tThese authors have contributed equally to this work

Specialty section:

This article was submitted to

Virus and Host,

a section of the journal

Frontiers in Cellular and Infection Microbiology

Received: 01 February 2020 Accepted: 24 March 2020

Published: 15 April 2020

Citation:

Hu Z, Pan Y, Cheng A, Zhang $X$ Wang $M$, Chen S, Zhu D, Liu M, Yang $Q$, Wu Y, Zhao X, Huang J, Zhang S, Mao S, Ou X, Yu Y, Zhang L,

Liu Y, Tian B, Pan L, Rehman MU, Yin Z and Jia R (2020) Autophagy Is a

Potential Therapeutic Target Against Duck Tembusu Virus Infection in vivo.

Front. Cell. Infect. Microbiol. 10:155.

doi: 10.3389/fcimb.2020.00155

\section{Autophagy Is a Potential Therapeutic Target Against Duck Tembusu Virus Infection in vivo}

\author{
Zhiqiang $\mathrm{Hu}^{1,2,3 \dagger}$, Yuhong Pan ${ }^{1,2,3 \dagger}$, Anchun Cheng ${ }^{1,2,3 *}$, Xingcui Zhang ${ }^{1,2,3}$, \\ Mingshu Wang ${ }^{1,2,3}$, Shun Chen ${ }^{1,2,3}$, Dekang Zhu ${ }^{1,2,3}$, Mafeng Liu ${ }^{1,2,3}$, Qiao Yang ${ }^{1,2,3}$, \\ Ying Wu ${ }^{1,2,3}$, Xinxin Zhao ${ }^{1,2,3}$, Juan Huang ${ }^{1,2,3}$, Shaqiu Zhang ${ }^{1,2,3}$, Sai Mao ${ }^{1,2,3}$, \\ Xumin Ou ${ }^{1,2,3}$, Yanling Yu ${ }^{1,2,3}$, Ling Zhang ${ }^{1,2,3}$, Yunya Liu ${ }^{1,2,3}$, Bin Tian ${ }^{1,2,3}$, Leichang Pan ${ }^{1,2,3}$, \\ Mujeeb Ur Rehman ${ }^{1,2,3}$, Zhongqiong Yin ${ }^{3}$ and Renyong Jia ${ }^{1,2,3 *}$

\begin{abstract}
${ }^{1}$ Institute of Preventive Veterinary Medicine, Sichuan Agricultural University, Wenjiang, China, ${ }^{2}$ Avian Disease Research Center, College of Veterinary Medicine of Sichuan Agricultural University, Wenjiang, China, ${ }^{3}$ Key Laboratory of Animal Disease and Human Health of Sichuan Province, Sichuan Agricultural University, Wenjiang, China
\end{abstract}

Duck tembusu virus (DTMUV) is newly emerged in poultry and causes great losses to the breeding industry in China and neighboring countries. Effective antiviral strategies are still being studied. Autophagy is a cellular degradative pathway, and our lab's previous data show that autophagy promotes DTMUV replication in vitro. To study the role of autophagy further in vivo, we utilized ducks as the animal model to investigate the autophagy responses in DTMUV-targeted tissues. And also, we utilized autophagy regulators, including Rapamycin (Rapa) as the autophagy enhancer, 3Methyladenine (3-MA) and Chloroquine (CQ) as the autophagy inhibitors, to adjust the host autophagic levels and then study the effects of autophagy on tissue damages and virus replication. As a result, we first found DTMUV infection trigged autophagy and autophagy regulator treatments regulated autophagy levels successfully in duck spleens and brains. Next, we found that autophagy inhibitors inhibited DTMUV replication and alleviated DTMUV-induced pathological symptoms, whereas the autophagy inducer treatment led to the opposite effects. And we also found that autophagic regulation was correlated with the expression of innate immune genes, including pattern recognition receptors, type I interferons, and cytokines, and caused different effects in different tissues. In summary, we demonstrated that autophagy facilitated DTMUV replication, aggravated the developments of pathological symptoms and possibly counteracts the host's innate immunity response in vivo.

Keywords: DTMUV, autophagy, spleen, brain, tissue damage, replication, immune response

\section{INTRODUCTION}

Autophagy is a cellular degradative pathway that delivers intracellular senescent organelles, longlived proteins, and exogenous pathogenic microorganisms to lysosomes for degradation (Klionsky and Emr, 2000). When cells are exposed to environmental stresses such as starvation, lack of growth factors, energy requirements, or invasion by pathogenic microorganisms, autophagy will be activated as a defense strategy to maintain the homeostasis of the intracellular environment 
(Chun and Kim, 2018). Autophagy is a dynamic process, including the formation of autophagosomes and the degradation of autophagosomes in lysosomes. One of the key signals for the formation of autophagosome is the mammalian target of rapamycin complex 1 (mTORC1), which is suppressive for autophagy (Klionsky et al., 2016). Therefore, some mTOR inhibitors have been used as autophagy inducers, such as Rapa (Jung et al., 2010) and Everolimus (Crazzolara et al., 2009). Another critical signal is phosphatidylinositol 3-kinases (PI3K), which is required for autophagy. And some PI3K inhibitors have been used as autophagy inhibitors, such as 3-MA (Wu et al., 2010), wortmannin (Blommaart et al., 1997), and 2-(4- morpholinyl)-8-phenyl-4H-1-benzopyran-4 one (LY294002) (Blommaart et al., 1997). There are also some autophagy inhibitors targeting the process of autophagic degradation. For instance, Chloroquine (CQ) has been reported to inhibit autophagy by raising the lysosomal $\mathrm{pH}$, which leads to inhibition of both fusion of autophagosome with lysosome and lysosomal protein degradation (Shintani and Klionsky, 2004). Bafilomycin A1 also inhibits autophagic degradation by inhibiting fusion between autophagosomes and lysosomes (Yamamoto et al., 1998).

Duck Tembusu virus (DTMUV), a member of the Flavivirus genus within the Flaviviridae family, is newly emerged in ducks in China (Su et al., 2011; Yan et al., 2011). Duck is the typical host of DTMUV, and some other species, like chickens, geese, and sparrows, also have been reported to be infected with DTMUV (Yu et al., 2018). Seriously, there might be high risk for poultry-to-human or mice transmission of the DTMUV (Liu et al., 2013; Tang et al., 2013). Therefore, it is emergency to study the DTMUV-host interaction and develop effective antivirus therapies. Multiple evidence has shown that the duck spleen is the target organ of DTMUV (Li et al., 2015; Sun et al., 2019b). Moreover, DTMUV has been reported to cause neurologic dysfunction (Thontiravong et al., 2015; Lv et al., 2019), which is similar to the neurological symptom caused by other flavivirus (Mustafá et al., 2019). And the presence of DTMUV has been detected in the duck brain (Li et al., 2015; Lv et al., 2019), which indicates that the duck brain is another target organ of DTMUV.

Multiple evidence has indicated that autophagy plays an important role in flavivirus infection (Ke, 2018). But there are rare reports on the effect of autophagy on virus replication in vivo. In this study, we first utilized ducks as the animal model to invested the role of autophagy in DTMUV infection in vivo.

\section{MATERIALS AND METHODS}

\section{Ethics Statement}

All animal experiments have been approved by the committee of experiment operational guidelines and animal welfare of Sichuan Agricultural University, China (the approved permit number is XF2014-18) and were performed in accordance with the relevant guidelines and regulations of National Institutes of Health. All surgeries were performed under sodium anesthesia with pentobarbital and every effort was made to relieve the suffering.

\section{Animals and Virus}

One-day-old Cherry Valley ducks were purchased from a farm operated by Sichuan Agricultural University (Sichuan, China) and housed in isolators until use. DTMUV-free ducks were confirmed by an immunochromatographic strip (ICS) developed by our lab (Deng et al., 2017). The DTMUV CQW1 strain (GenBank: KM233707.1) used in this study was isolated from a young duck in Southwest China and purified by the plaque method in our laboratory (Zhu et al., 2015).

\section{Experimental Design}

Twenty-five 7-day-old ducks were randomly divided into 5 groups ( $n=5$ /each group). The ducks in group $2,3,4$, and 5 were infected with 400,000 TCID50 viruses by intramuscular injection, and then treated with saline, rapamycin (Rapa, 2 $\mathrm{mg} / \mathrm{kg}$ of body weight), 3-Methyladenine (3-MA, $2 \mathrm{mg} / \mathrm{kg}$ ), or Chloroquine (CQ, $20 \mathrm{mg} / \mathrm{kg}$ ) by intraperitoneal injection, respectively. The pharmaceutical treatments were carried out $2 \mathrm{~h}$ after virus infection, which was followed by treatments with drugs or saline every $12 \mathrm{~h}$. The ducks in group 1 were treated with saline as the control. At $72 \mathrm{~h}$ posttreatment, these ducks were euthanized and duck tissues were collected for different goals with different protocols as followed.

\section{Antibodies and Chemicals}

The primary antibodies of anti-LC3 (14600-1-AP) and anti- $\beta$ actin (60008-1-Ig), were purchased from Proteintech (Wuhan, Hubei, China). Anti-SQSTM1/p62 (5114) was purchased from Cell Signaling Technology (Danvers, Massachusetts, USA). The monoclonal antibody against the DTMUV E protein was prepared in our laboratory. Horseradish peroxidases (HRP) conjugated to goat anti-mouse secondary antibodies (BF03001) were purchased from Beijing Biodragon Immunotechnologies (Beijing, China). Rapamycin (Rapa) (HY-10219), 3-Methyladenine (3-MA) (HY-19312), chloroquine (CQ) (HY-17589), and were purchased from MedChemExpress (MCE, Monmouth Junction, New jersey, USA).

\section{Western Blotting (WB)}

Hundred milligram of spleens specimens and brains specimens were weighed and then immediately cryopreserved in liquid nitrogen until being processed for protein isolation. When processed for protein isolation, spleen tissues and brain tissues were homogenized and then lysed with RIPA lysis buffer (Solarbio, R0020, Beijing, China) containing $1 \mathrm{mM}$ phenylmethylsulfonyl fluoride (PMSF, an inhibitor of serine proteases and acetylcholinesterase) (Boster, AR1178, Beijing, China). The concentration of extractive protein was measured using a BCA protein assay kit (Solarbio, PC0020, Beijing, China). Equal amounts of protein samples were boiled for $5 \mathrm{~min}$ in 4 $\times$ SDS-PAGE loading buffer, separated on 12-15\% SDS-PAGE gels, and then electrotransferred onto polyvinylidene fluoride (PVDF) membranes (BIO-RAD, 162-0177, Hercules, California, USA). The PVDF membranes with the target proteins were then blocked for $2 \mathrm{~h}$ at room temperature in Tris-Buffered Saline and Tween 20 (TBST) containing 5\% non-fat milk powder. After that, the membranes were incubated with anti-LC3 (1:1000), anti-p62 
$(1: 1000)$ and anti- $\beta$-actin $(1: 2000)$ antibodies at $4{ }^{\circ} \mathrm{C}$ overnight and then with the corresponding secondary antibodies (1:5000), conjugated to $\mathrm{HRP}$ at $37{ }^{\circ} \mathrm{C}$ for $1 \mathrm{~h}$. The protein bands were developed by an ECL Plus kit (Solarbio, PE0010, Beijing, China) and imaged by ChemiDoc MP (Bio-Rad, Hercules, California, USA). The densitometry of WB bands was measured by the Image Lab software.

\section{Hematoxylin and Eosin (HE) Staining and Immunohistochemistry (IHC)}

The spleen tissues and brain tissues were fixed in $4 \%$ paraformaldehyde, and then enclosed in paraffin-intended subsequent histopathological examination. A $4 \mu \mathrm{m}$ section of each tissue was stained with hematoxylin and eosin. Each section was examined under an optical microscope. IHC was conducted as described previously (Ou et al., 2017). Briefly, slides were boiled in Tris/EDTA pH 9.0 for $20 \mathrm{~min}$. Then, $0.01 \mathrm{M}$ $\mathrm{HCl}$ was used to block endogenous alkaline phosphatase for $15 \mathrm{~min}$ at room temperature (RT). Then, $3 \% \mathrm{H}_{2} \mathrm{O}_{2}$ was used to block endogenous peroxidase for $15 \mathrm{~min}$ at RT. The slides were incubated in 5\% BSA blocking solution followed by overnight incubation at $4{ }^{\circ} \mathrm{C}$ in mouse anti-DTMUV-E polyclonal antibody (1:20 dilutions). HRP coupled goat anti-mouse IgG (1:1000 dilutions) was incubated for $30 \mathrm{~min}$ at $37^{\circ} \mathrm{C}$. Then, positive staining was colored with DAB solution for $10 \mathrm{~min}$ at RT and counterstained with hematoxylin.

\section{Quantitative RT-PCR Assay (qRT-PCR)}

Hundred milligram of spleen specimens and brains specimens were collected with the same procedure as previously described in the section of "Western blotting." Total cellular RNA was isolated from $100 \mathrm{mg}$ of tissue specimens using the RNAiso plus Reagent (TaKaRa, Japan), and subsequently transcripted into cDNA using PrimeScript ${ }^{\mathrm{TM}}$ RT reagent Kit (Takara, RR047A, Dalian, China) according to the manufacturer's protocol. The mRNA levels of immune genes, including RIG-I, MDA5, TLR3, IFN- $\alpha$, IFN- $\beta$, IFN- $\gamma$, IL-1 $\beta$, IL- 6 , and IL- 8 , were detected by qRT-PCR which was performed using the Bio-Rad CFX96 RealTime Detection System (Bio-Rad, USA), and the $\beta$-actin was as the housekeeping gene. Additionally, viral copies were detected by previously established methods in our laboratory (Zhang et al., 2019b). The sequences of the gene-specific primers used for qRT-PCR are shown in Table 1.

\section{Statistical Analysis}

Data are presented as means \pm standard deviations (SD). The significance of the variability between different treatment groups was analyzed by the two-tailed independent Student $t$-test using the GraphPad Prism software (version 6.0). A $P<0.05$ was considered to be statistically significant.

\section{RESULTS}

\section{DTMUV Infection Enhances Autophagy in Duck Spleens and Brains}

Our previous data have shown that DTMUV infection triggered autophagy in vitro. To further know the role of autophagy
TABLE 1 | Primer sequences used in this study.

\begin{tabular}{|c|c|}
\hline Prime name & Prime sequence $\left(5^{\prime}-3^{\prime}\right)$ \\
\hline Duck Rig-I-F & GCGTACCGCTATAACCCACA \\
\hline Duck Rig-I-R & CCTTGCTGGTITGAACGC \\
\hline Duck MDA5-F & GCTGAAGAAGGCCTGGACAT \\
\hline Duck MDA5-R & TCCTCTGGACACGCTGAATG \\
\hline Duck TLR3-F & GAGTITCACACAGGATGTITAC \\
\hline Duck TLR3-R & GTGAGATTGTTCCTTGCAG \\
\hline Duck IFN- $\alpha-F$ & TCСТССАACACСТСТTСGAC \\
\hline Duck IFN- $\alpha-R$ & GGGCTGTAGGTGTGGTTCTG \\
\hline Duck IFN- $\beta-F$ & AGATGGCTCCCAGCTCTACA \\
\hline Duck IFN- $\beta-R$ & AGTGGTTGAGCTGGTTGAGG \\
\hline Duck IFN- $\gamma-F$ & GCTGATGGCAATCCTGTIT \\
\hline Duck IFN- $\gamma$ R & GGATITTCAAGCCAGTCAGC \\
\hline Duck IL-1 $\beta-F$ & TCATCTTCTACCGCCTGGAC \\
\hline Duck IL-1 $\beta-R$ & GTAGGTGGCGATGTTGACCT \\
\hline Duck IL-6-F & TTCGACGAGGAGAAATGCTT \\
\hline Duck IL-6-R & CCTTATCGTCGTTGCCAGAT \\
\hline Duck IL-8-F & AAGTTCATCCACCCTAAATC \\
\hline Duck IL-8-R & GCATCAGAATTGAGCTGAGC \\
\hline Duck $\beta$-actin-F & GGTATCGGCAGCAGTCTTA \\
\hline Duck $\beta$-actin $\mathrm{R}$ & TTCACAGAGGCGAGTAACTT \\
\hline DTMUV-Cap-F & AGGTITGTGCTGGCTCTAC \\
\hline DTMUV-Cap-R & TGTTTGGTCGCCTCATT \\
\hline
\end{tabular}

in vivo, we tested the protein levels of autophagy makers, including the conversion from LC3-I to LC3-II and the degradation of p62, in DTMUV-targeted organs. Duck spleens and brains have been reported to be the target organs in DTMUV infection (Li et al., 2015; Thontiravong et al., 2015; Lv et al., 2019; Sun et al., 2019b). As shown in Figure 1, DTMUV infection increased the protein levels of LC3-II but decreased p62 levels compared to those in the control group, which indicated that DTMUV enhanced both autophagic initiation and autophagic flux in spleens (Figure 1A) and brains (Figure 1B). We also examined the formations of autophagosome-like vesicles in the two organs by TEM. Many double-membrane structures, including autophagosome-like (Figures S1C,G) and autolysosome-like vesicles (Figures S1D,H), were observed in the spleens and brains of DTMUV-infected ducks (Figures S1B,F), whereas few double-membrane structures observed in the organs of salinetreated ducks (Figures S1A,E). Furthermore, we found that Rapa treatment further improved LC3-II increase and p62 decrease in both spleens and brains (Figures 1A,B), which indicated the promotion of autophagic activation. 3-MA treatment, an inhibitor of autophagy initiation, decreased the levels of LC3-II but increased p62 levels in spleens compared to those in DTMUV-infected ducks with saline treatment. Whereas, there were decreased levels of LC3-II, but no significant change of p62 levels in brains (Figure 1B). CQ treatment enhanced the accumulation of both LC3-II and p62, which indicated that DTMUV-trigged autophagic flux was inhibited in both spleens and brains. 

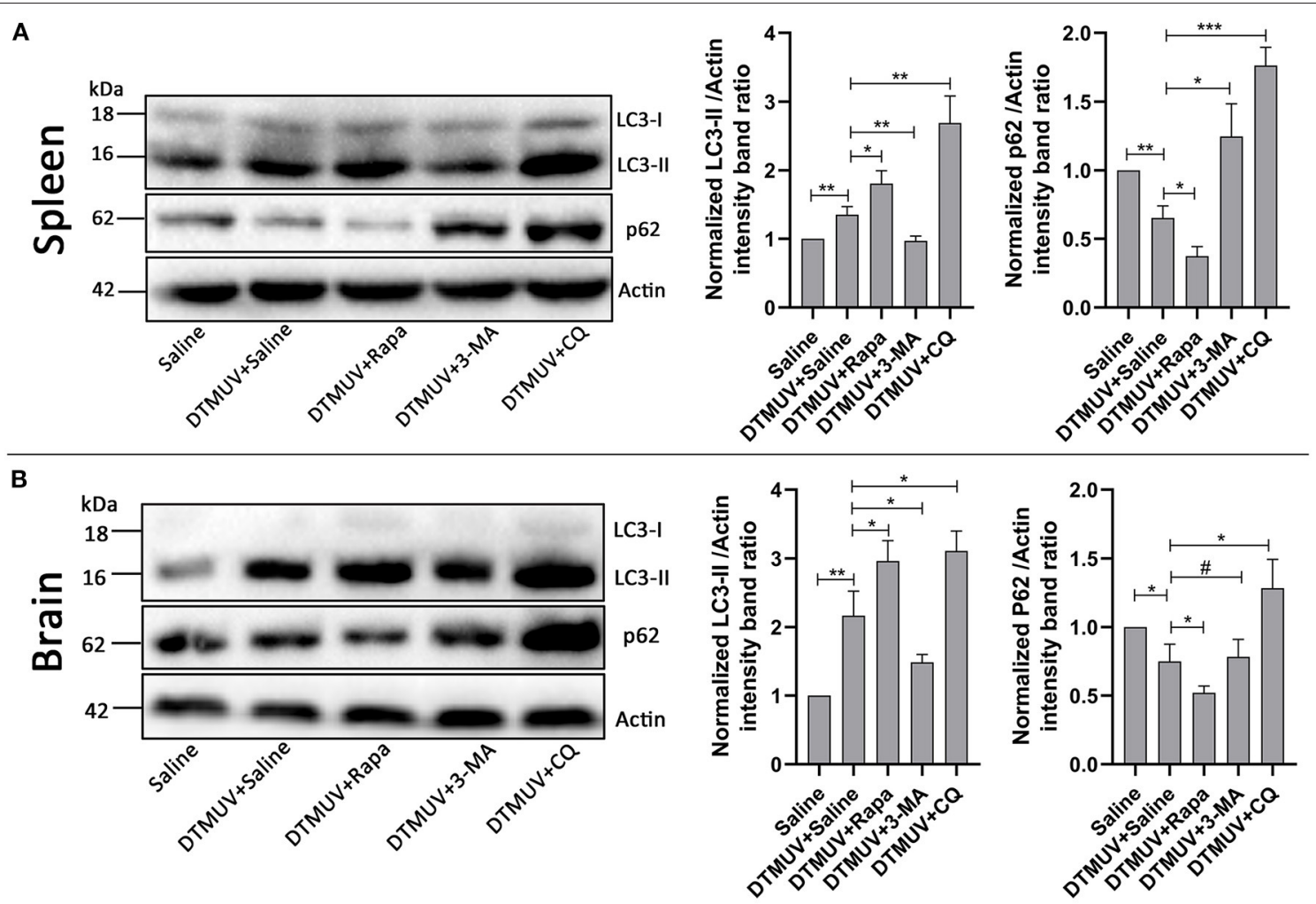

FIGURE 1 | Measurement of autophagic markers in the duck spleens and brains. The protein levels of LC3 and p62 in duck spleens (A) and duck brains (B) were analyzed by Western blot assay. The ratios of targeting proteins to $\beta$-actin were normalized to those in the saline group. Data are expressed as means \pm standard deviations $(n=5)$. Differences were evaluated with Two-tailed Student's $t$-test. ${ }^{\#} p>0.05,{ }^{*} p<0.05,{ }^{* \star} p<0.01,{ }^{\star \star *} p<0.001$.

\section{Autophagy Regulators Affect the Gross Pathology of Spleens and Brains in DTMUV-Infected Ducks}

To investigate the effect of autophagy on the gross pathology of spleens and brains in DTMUV-infected ducks, we observed the gross pathologies of the two tissues with different autophagy regulator treatments. As shown in Figure 2A, the normal spleen was with normal size and in pale red. DTMUV infection caused the spleen to be swollen and in dark red. Rapa treatment aggravated these symptoms, whereas 3-MA and CQ treatments relieved them. As for brains, as shown in Figure 2B, the normal brain was in light pink and with few blood streaks on the meninx. DTMUV infection caused meninx congestion, and Rapa treatment made the brain dark pink, which indicated more serious symptoms in the brain. Whereas, there were fewer blood streaks on the brains with 3-MA and CQ treatments, which indicated that the symptom of meninx congestion was relieved.

\section{Autophagy Regulators Affect the Histopathologic Lesions of Spleens and Brains in DTMUV-Infected Ducks}

We further analyzed the histopathologic lesions of spleens and brains. As shown in Figure $\mathbf{3 A}$, there were normal structures of the spleens in the only saline-treated ducks, including white pulps (WPs), red pulps (RPs), and the clear boundary line between WPs and RPs. DTMUV infection caused an obvious increase of red blood cells in RPs, which indicated the spleen congestion and hemorrhage. This result might explain the dark red color of spleens with DTMUV infection (Figure 2A). Rapa treatment aggravated these pathological changes, and it was hard to observe the boundary line between WPs and RPs in these spleens. On the contrary, there were just slight congestions in the spleens with 3-MA treatments and CQ treatments, which indicated that 3-MA and CQ alleviated these pathological symptoms caused by DTMUV infection.

As for brains (Figure 3B), there were normal meninx structure, and no blood cells observed under the meninx. While the space under meninx was dilated and amounts of blood cells were observed in that in DTMUV-infected brains. And Rapa treatment further aggravated the histopathologic lesions induced by DTMUV infection. There was a bigger space under meninx and the blood might outflow of the meninx in the DTMUV-infected brains with Rapa treatment. On the contrary, there were just slight swellings of meninges and a few blood cells under the meninges in the DTMUV-infected brains with 3-MA treatments and CQ treatments. 


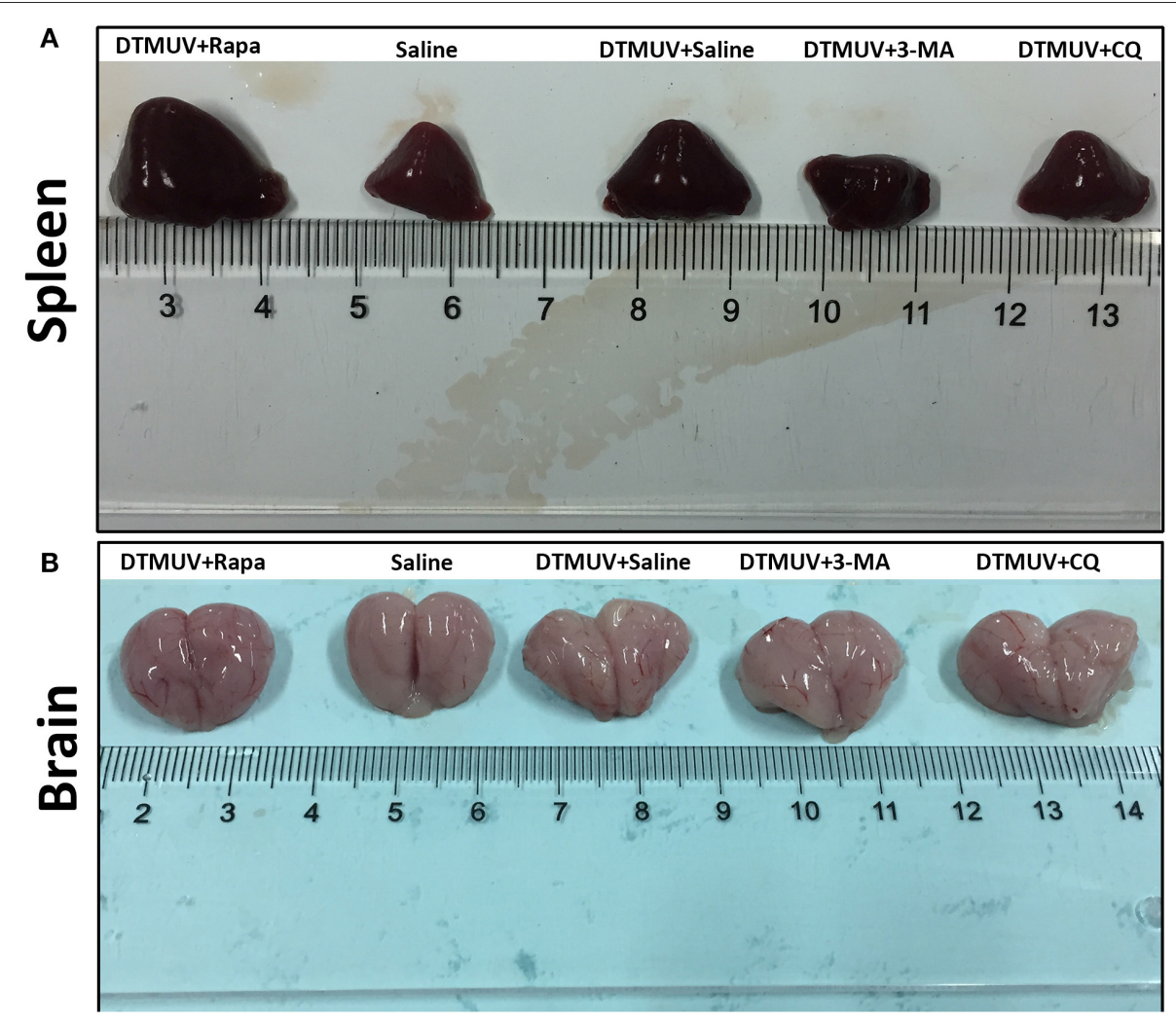

FIGURE 2 | Gross pathology of the spleens (A) and the brains (B) of ducks infected with DTMUV in the absence or presence of either Rapa, 3-MA, or CQ, respectively. Treatment with saline was used as the control. Images shown were representative from five ducks in each group.

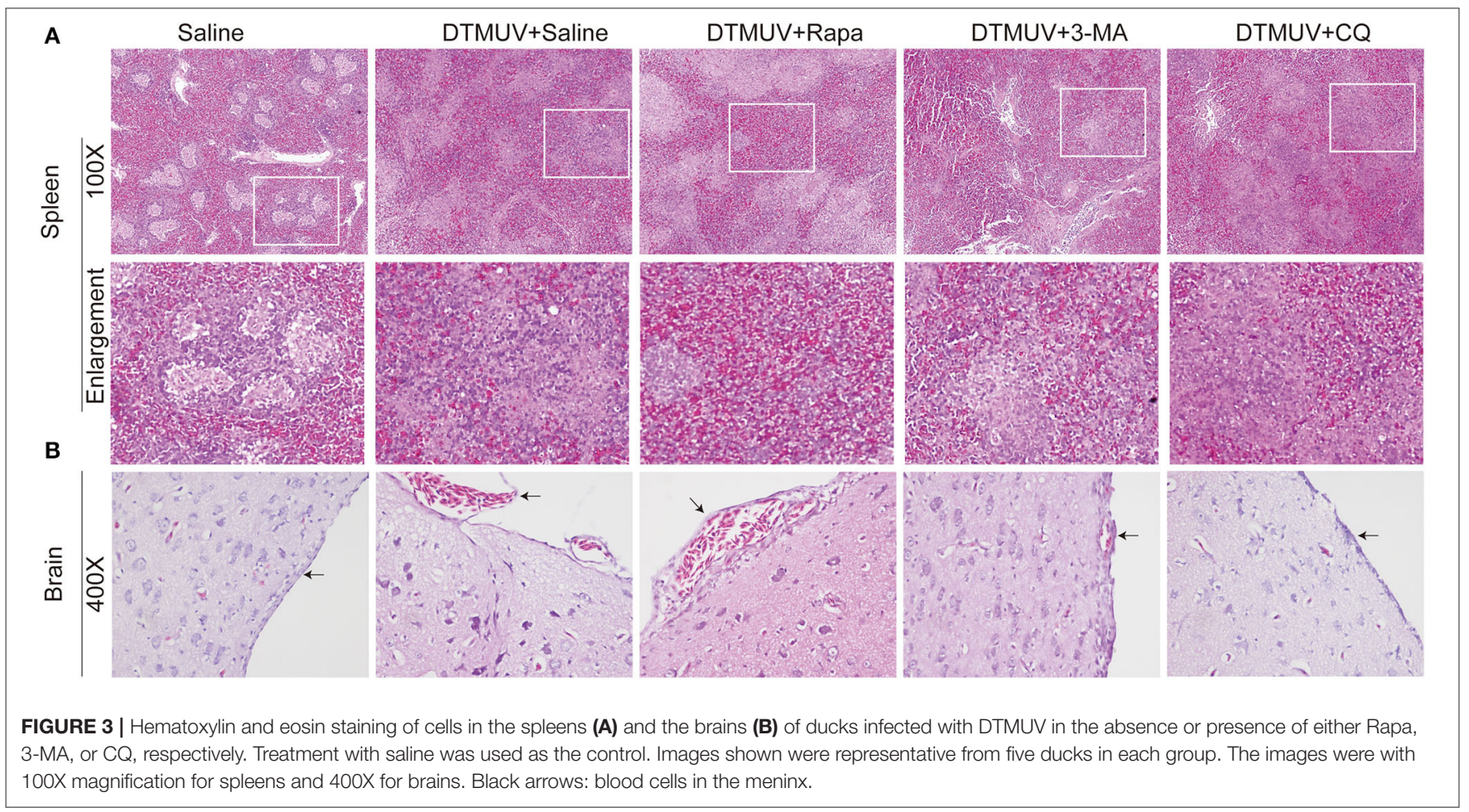


A

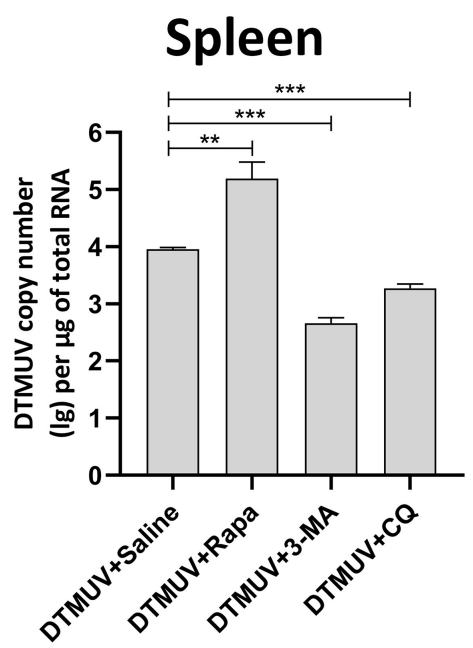

B

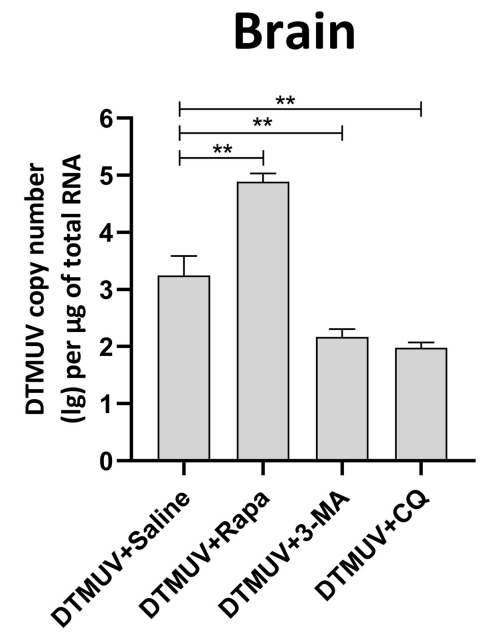

C

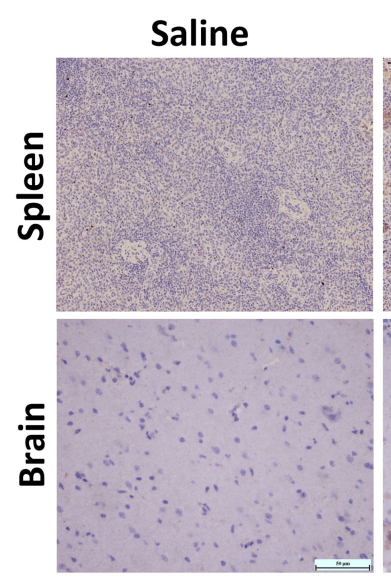

DTMUV+Saline

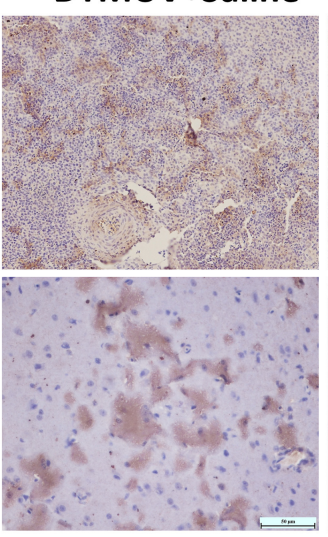

DTMUV+Rapa

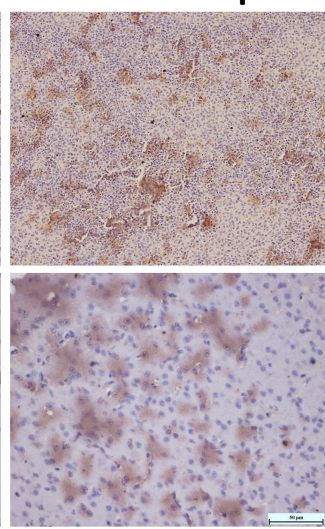

DTMUV+3-MA

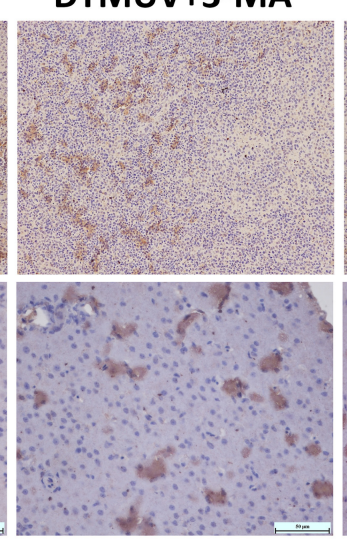

DTMUV+CQ

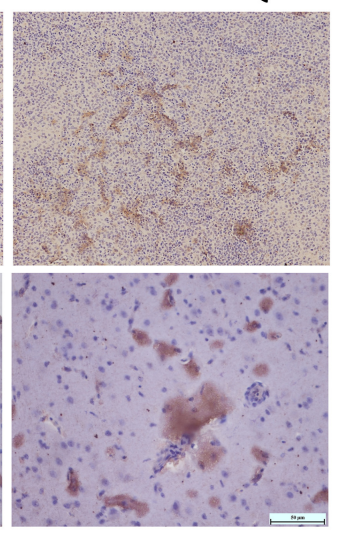

FIGURE 4 | The replication of DTMUV in the spleens and the brains of ducks infected with DTMUV in the absence or presence of either Rapa, 3-MA, or CQ, respectively. (A,B) The viral titers in the spleens $\mathbf{( A )}$ and the brains $\mathbf{( B )}$ were analyzed by qRT-PCR and expressed as means \pm standard deviations $(n=5)$. Differences were evaluated with Two-tailed Student's $t$-test. ${ }^{\star *} p<0.01,{ }^{\star \star *} p<0.001$. (C) The expression of DTMUV E protein was tested by IHC. Images shown were representative from five ducks in each group.

\section{Autophagy Regulators Affect the Replication of DTMUV in Duck Spleens and Brains}

Generally, the levels of pathological symptoms were always related to the amount of virus in tissues. Then, we analyzed DTMUV replication in spleens and brains of ducks with autophagy regulator treatments. First, the virus titers were analyzed by qRT-PCR. As shown in Figures $4 A, B$, the results showed that virus titers were increased significantly in both spleens and brains with Rapa treatments, whereas decreased with 3-MA and CQ treatments compared to that in the only salinetreated group. Furthermore, the expression of envelope protein of DTMUV was tested by IHC in the two tissues. As shown in Figure 4C, many E-positive cells were observed in DTMUVinfected spleens and brains with Rapa treatment or not. While, there was a decreased number of E-positive cells in 3-MA-treated and CQ-treated tissues. No E-positive cells were observed in only saline-treated spleens or brains.

\section{Autophagy Regulators Affect the Innate Immune Responses of Spleens and Brains in DTMUV-Infected Ducks}

Autophagy has been reported to play essential roles in virus infection and host immune responses. To investigate the effects of autophagy on the innate immune responses with DTMUV infection in vivo, we tested the mRNA expression levels of pattern recognition receptors (PRRs, including RIG-1, MDA5, and TLR3), interferons (IFNs, including IFN- $\alpha$, IFN- $\beta$, and IFN- $\gamma$ ) and cytokines (IL-1 $\beta$, IL-6, IL-8), in duck spleens and brains.

\section{Expression of Immune Genes in the Spleens}

Among the PRRs expressed in the spleens (Figure 5A), DTMUV infection increased the mRNA levels of RIG-I, MDA5, and TLR3 significantly. And we further found that the levels of RIG-I and MDA5 were inhibited with Rapa treatment, whereas enhanced with 3-MA and CQ treatments in DTMUV-infected spleens, as 
A
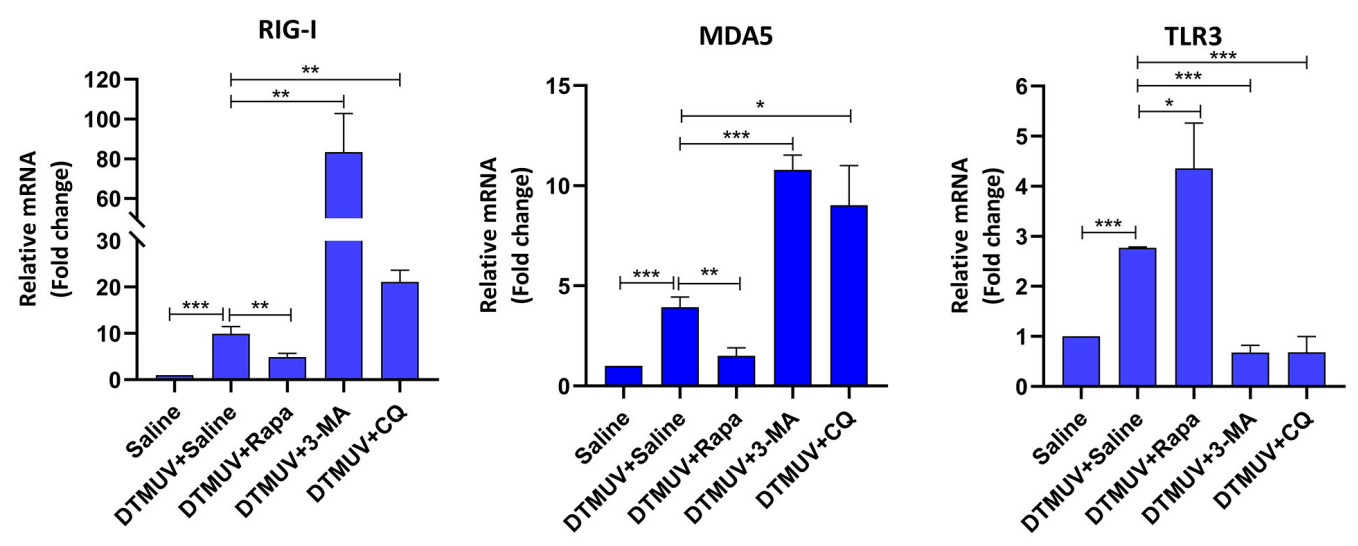

B
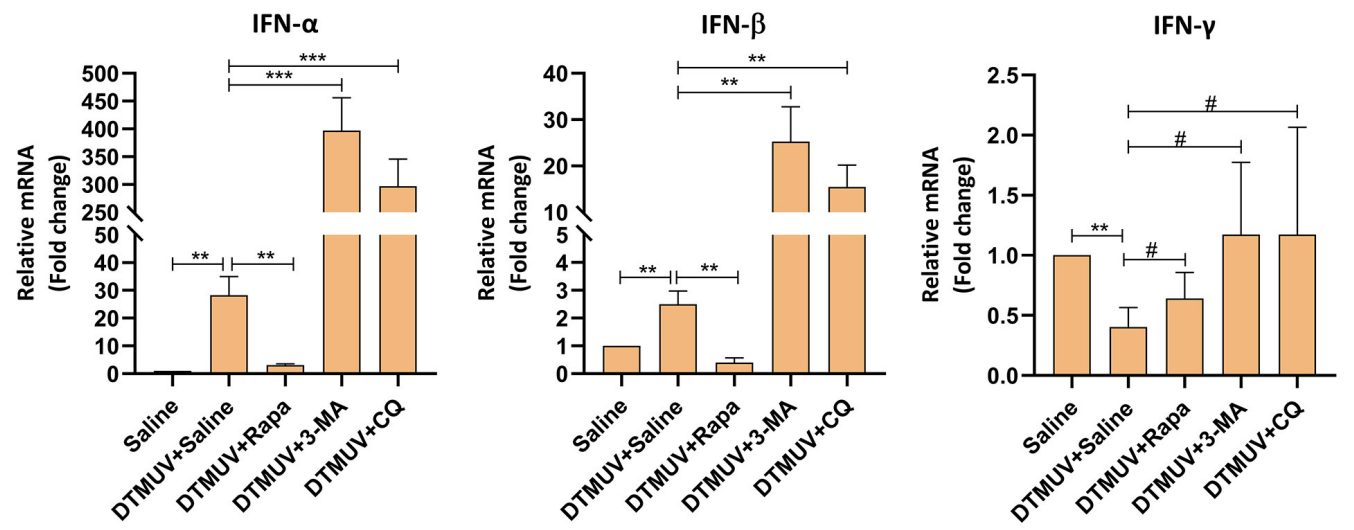

C
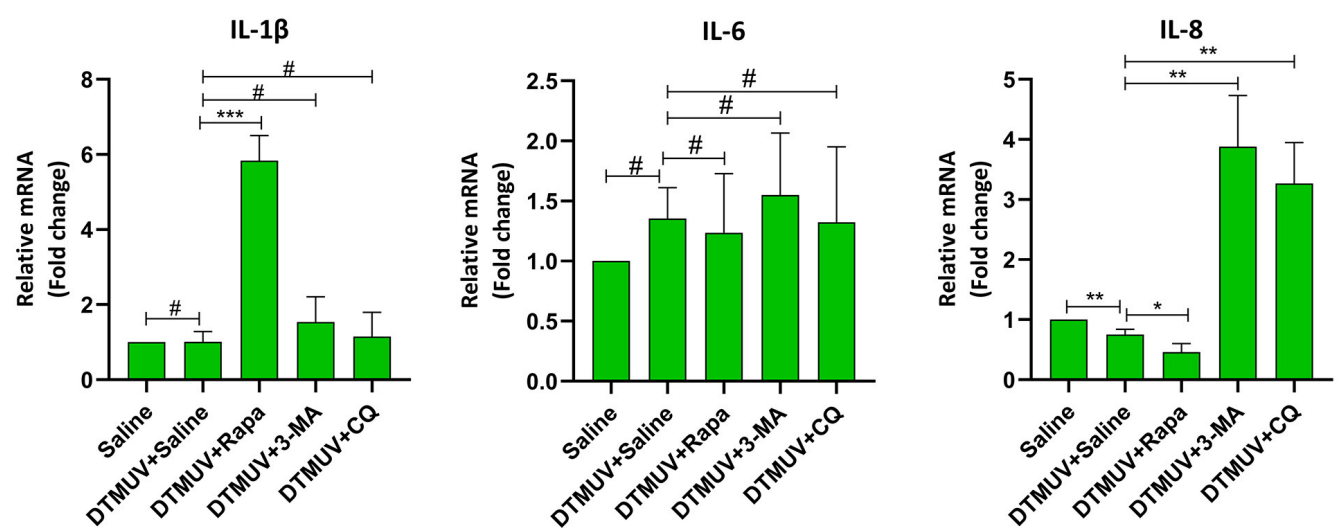

FIGURE 5 | Expression of immune-related genes in the spleens of ducks infected with DTMUV in the absence or presence of either Rapa, 3-MA, or CQ, respectively. (A) The mRNA levels of PRRs, including RIG-I, MDA5, and TLR3. (B) The mRNA levels of IFNs, including IFN- $\alpha$, IFN- $\beta$, and IFN- $\gamma$. (C) The mRNA levels of pro-inflammatory cytokines, including IL-1 $\beta, I L-6, I L-8$. Treatment with saline was used as the control. Data are expressed as means \pm standard deviations $(n=5)$. Differences were evaluated with Two-tailed Student's $t$-test. ${ }^{\#} p>0.05,{ }^{*} p<0.05$, ${ }^{\star \star} p<0.01,{ }^{\star \star \star} p<0.001$.

compared to those with saline treatment. Besides, Rapa treatment increased the mRNA levels of TLR3, while 3-MA and CQ treatments decreased it compared to that in saline treatment. These results indicated that autophagy played a positive role in the expression of RIG-1 and MDA5, but a negative role in TLR3.

Among the IFNs expressed in the spleens (Figure 5B), DTMUV infection increased the mRNA levels of IFN- $\alpha$ (28.23fold) and IFN- $\beta$ (2.5-fold) significantly, in particular a bigger increase of the IFN- $\alpha$ level compared to that of IFN- $\beta$. Whereas, the level of IFN- $\gamma$ was decreased in DTMUV-infected spleens. Furthermore, we found that the levels of IFN- $\alpha$ and IFN- $\beta$ were inhibited with Rapa treatment, whereas enhanced with 3-MA and CQ treatments in DTMUV-infected spleens, as compared to those with saline treatment. But there were no significant effects on IFN- $\gamma$ levels in the spleen tissue with autophagy regulator treatments. 
Among the cytokines expressed in spleen tissues (Figure 5C), DTMUV infection only decreased the mRNA level of IL-8 significantly, whereas no effects on IL- $1 \beta$ and IL-6. We also found that Rapa treatment further inhibited the level of IL-8, while 3-MA and CQ treatments enhanced that compared to saline treatment, which indicated that the level of IL- 8 was correlated to the autophagy responses. In addition, 3-MA and CQ treatments have no effects on the mRNA levels of IL-1 $\beta$ and IL-6, while Rapa treatment increased the levels of IL- $1 \beta$ significantly, but no effects on IL-6.

\section{Expression of Immune Genes in the Brains}

Among the PRRs expressed in brain tissues (Figure 6A), DTMUV infection increased the mRNA levels of RIG-I, MDA5, but decreased TLR3 significantly. And the level of RIG-I was inhibited with Rapa treatment, whereas enhanced with 3-MA and CQ treatments, as compared to saline treatment. In addition, Rapa treatment has no effects on the levels of MDA5 or TLR3. 3-MA treatment increased the levels of MDA5 but no effect on TLR3, whereas CQ treatments increased the levels of TLR3 but no effects on MDA5.

Among the IFNs expressed in brain tissues (Figure 6B), DTMUV infection increased the mRNA levels of IFN- $\alpha$ and IFN- $\gamma$ significantly, but no effects on IFN- $\beta$. And the level of IFN- $\alpha$ was inhibited with Rapa treatment while enhanced with 3-MA and CQ treatments, as compared to saline treatment. But there were no significant effects on IFN- $\beta$ levels with autophagy regulator treatments. In addition, Rapa treatments inhibited the level of IFN- $\gamma$, whereas 3-MA and CQ treatments have no effects on that.

Among the cytokines expressed in brain tissues (Figure 6C), DTMUV infection increased the mRNA levels of IL-6 and IL8 significantly, but no effects on IL-1 $\beta$. Furthermore, the level of IL-6 was inhibited with Rapa treatment, whereas enhanced with 3-MA and CQ treatments, as compared to saline treatment. In addition, Rapa treatment inhibited the level of IL- $1 \beta$ but no effects on IL-8. And 3-MA or CQ treatments have no effects on IL- $1 \beta$ and IL- 8 levels.

\section{DISCUSSION}

Multiple evidence suggests that autophagy plays a crucial role in the life cycles of flaviviruses in vitro and in vivo (Ke, 2018). As we have investigated that autophagy promotes the replication of DTMUV in vitro (Hu et al., 2020), to further provide the clinical evidence on the effects of autophagy on DTMUV replication and pathogenesis, we utilized ducks as the animal model to study the role of autophagy in DTMUV-targeted organs.

We first found that DTMUV infection triggered autophagy in duck spleens and brains which were the target organs of DTMUV (Figure 1 and Figure S1). Autophagy always occurs in the target organs of virus infection. For instance, Dengue virus (DENV), another flavivirus, also has been reported to trigger autophagy in mice brains (Lee et al., 2013). Newcastle Disease Virus (NDV) and Avian Influenza A H5N1, RNA virus of other species, also trigged autophagy in their target organs, respectively (Sun et al., 2012, 2014). The trigged-autophagy in target organs might be caused by the amount of virus replication in these organs. Moreover, to study the effects of autophagy on DTMUV pathogenicity in vivo, we utilized autophagy regulators to adjust the host autophagic levels. Rapa has been investigated to be an effective enhancer of autophagy in vivo, such as mice (Lee et al., 2013) chickens (Sun et al., 2014). Our result in Figure 1 showed the protocol of Rapa treatment in this study successfully increased the autophagic level. 3-MA inhibits autophagy by blocking autophagosome formation via the inhibition of class III PI3K (Klionsky et al., 2016) and has been used to inhibit the autophagy induced by various Flaviviruses, such as Zika Virus (ZIKV) in vitro (Cao et al., 2017), DENV in vitro (Lee et al., 2008) and in vivo (Lee et al., 2013), classical swine fever virus (CSFV) in vitro (Pei et al., 2014). And our previous data has shown 3-MA treatment successfully inhibits DTMUVinduced autophagy in vitro ( $\mathrm{Hu}$ et al., 2020). However, one case shows that prolonged treatment with 3-MA promotes autophagy under nutrient-rich conditions (Wu et al., 2010). But there is no evidence to show the promotion of 3-MA treatment in virus- or starvation-inducted autophagy. In this study, we treated ducks with 3-MA once every $12 \mathrm{~h}$ and for $72 \mathrm{~h}$, which had a good efficiency for the inhibition of DTMUVinducted autophagy in duck brains and spleens (Figure 1). And also, to eliminate the problem of the dual role of 3-MA in autophagy, we utilized another autophagy inhibitor, CQ, to block DTMUV-induced autophagy. CQ inhibits autophagy by decreasing autophagosome-lysosome fusion (Mauthe et al., 2018), and has been reported to block NDV-inducted autophagy in Chickens, which is a relative species to duck (Sun et al., 2014). In the current study, CQ enhanced the accumulation of LC3-II and p62 (Figure 1), indicting the successful inhibition of DTMUV-induced autophagy in ducks. Above all, the autophagy inducer and inhibitors adjusted autophagic levels in the two organs successfully, which meant that we could utilize these models to study the mechanism of the role of autophagy on DTMUV replication next.

DTMUV caused obvious gross pathologies and histopathologic lesions in duck spleens and brains (Figures 2, 3). These changes are consistent with other's reports ( $\mathrm{Lv}$ et al., 2019; Sun et al., 2019a). And we first found that the levels of autophagy were positively correlated with the degree of tissue damages induced by DTMUV infection. The therapeutic effects of autophagy inhibitors also have been reported in ZIKV (Zhang et al., 2019a), DENV (Lee et al., 2013), NDV (Sun et al., 2014), and H5N1 (Sun et al., 2012) infection in vivo. Moreover, the tissue damage of spleens indicated that DTMUV infection might cause damage to the host immune system. Multiple evidence indicates that autophagy is a tool to regulate the innate immune system (Germic et al., 2019). So, autophagy inhibitors might alleviate spleen damage by adjusting the levels of innate immune responses. Like some other flaviviruses (Mustafá et al., 2019), DTMUV infection also counteracted the Blood-Brain Barrier and invaded the Central Nervous System (CNS). There is an opinion that the pathogenesis of CNS is always related to impaired autophagy (Nikoletopoulou et al., 2015). Our results also showed that brain damage was related to the levels of autophagy. We 
A
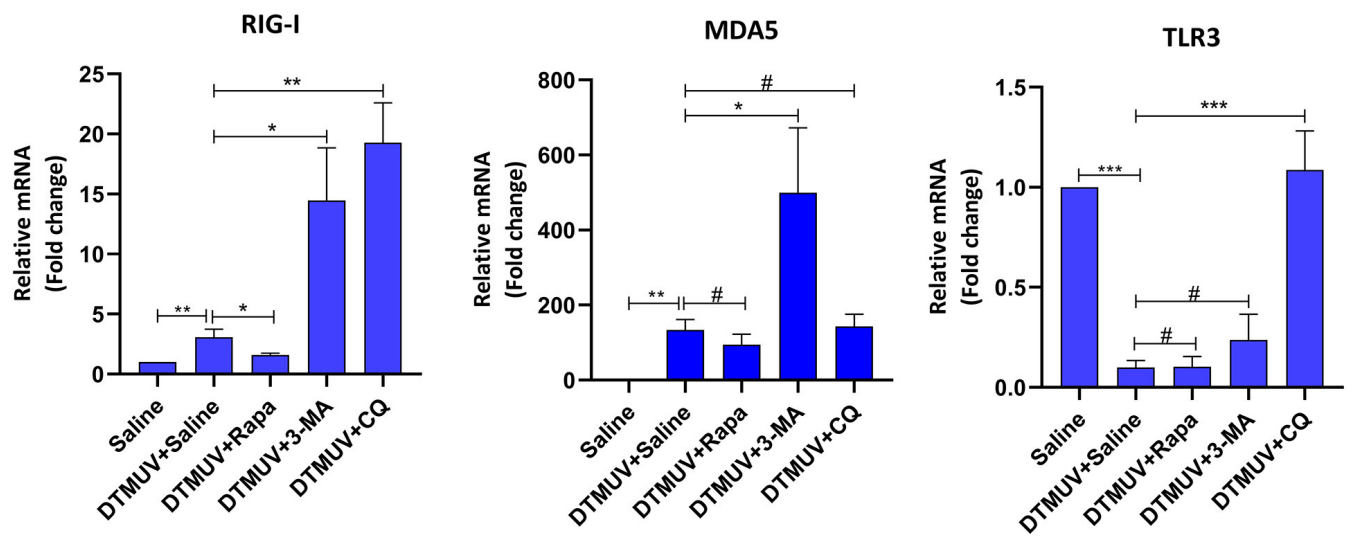

B
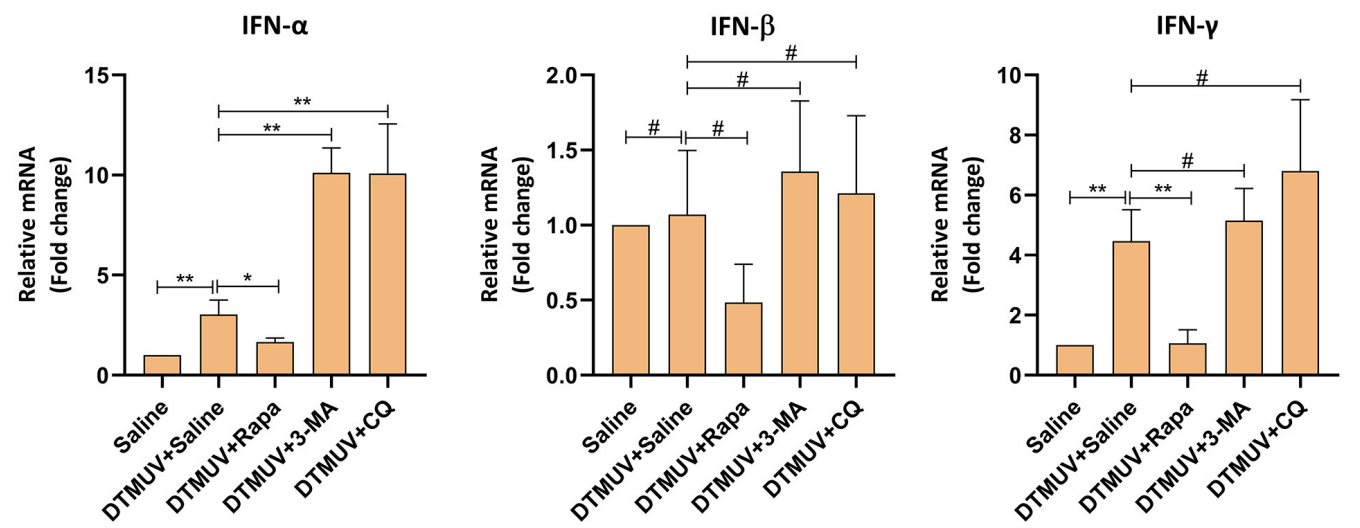

c
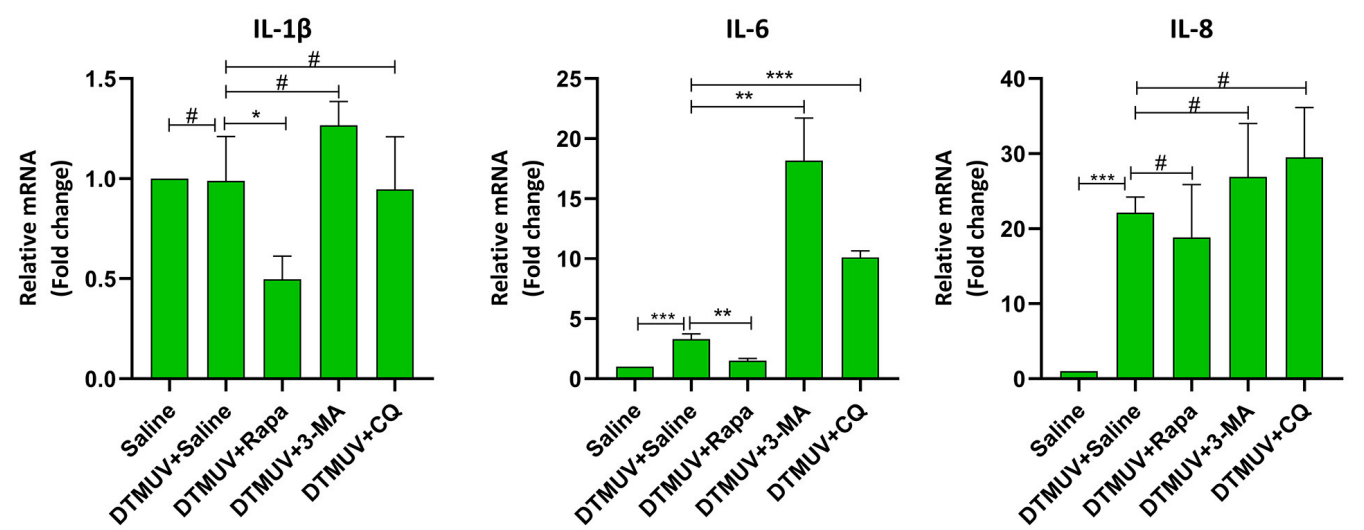

FIGURE 6 | Expression of immune-related genes in the brains of ducks infected with DTMUV in the absence or presence of either Rapa, 3-MA, or CQ, respectively. Treatment with saline was used as the control. (A) The mRNA levels of PRRs, including RIG-I, MDA5, and TLR3. (B) The mRNA levels of IFNs, including IFN- $\alpha$, IFN- $\beta$, and IFN- $\gamma$. (C) The mRNA levels of cytokines, including IL-1 $\beta, I L-6, I L-8$. Data are expressed as means \pm standard deviations ( $n=5)$. Differences were evaluated with Two-tailed Student's t-test. ${ }^{\#} p>0.05,{ }^{*} p<0.05,{ }^{* \star} p<0.01,{ }^{* \star *} p<0.001$.

provided some references for the therapeutic developments of flaviviruses-caused neurologic symptoms.

The levels of tissue damage are always related to virus replication in the corresponding tissues. In the current study, we found that autophagy regulator treatments affected DTMUV replication significantly in the target tissues. The formation of autophagosomes has been reported to be required for the maturation of infectious dengue virus production (Mateo et al., 2013). And also, autophagosomes supply places for the formation of membrane structures of hepatitis $\mathrm{C}$ virus (HCV) (Mohl et al., 2016). Besides, some flaviviruses, including HCV (Ren et al., 2016; Shrivastava et al., 2016), DENV (McLean et al., 2011), and Japanese encephalitis virus (Tasaki et al., 2016), use autophagic membranes for their release via the exosomal pathway through multivesicular bodies (Shrivastava et al., 2011; Metz et al., 2015), and these may be related 
to the process of the fusion between autophagosomes and lysosomes. Therefore, in this study, Rapa and 3-MA might affect the replication of DTMUV in target tissues by regulating the formation of autophagosomes. 3-MA also has been reported to reduce DENV replication in mice by autophagy pathway (Lee et al., 2013). CQ is an autophagy inhibitor by inhibiting the autophagosome-lysosome fusion and might reduce DTMUV replication by targeting this pathway. Similar results are also found in ZIKV-infected mice with CQ treatment (Zhang et al., 2019a). Moreover, our lab's previous data invested that p62 regulated the innate antiviral response in DTMUV-infected cells (Hu et al., 2020). Hence, CQ treatments might also inhibit DTMUV replication by causing the accumulation of p62, and then affecting the host antiviral response in vivo. In addition, CQ also has some antiviral activities by autophagyindependent pathways. CQ is weak base, and meddles in protein processing, and degradation by alkalifying the acidic organelles like Golgi vesicles, endosomes, lysosomes (Gratton et al., 2019). And the low $\mathrm{pH}$ is essential for the entry, the viral RNA release, and the exit of flaviviruses, like ZIKV, DENV, HCV, and others (Tscherne et al., 2006; Sánchez-San Martín et al., 2009; Zheng et al., 2014; Persaud et al., 2018). DTMUV also has been reported to enters BHK-21 cells by a low pHdependent endosomal pathway (Baloch et al., 2019). Therefore, CQ treatment might inhibit DTMUV replication by blocking the $\mathrm{pH}$-dependent stages of DTMUV replication. Further study needs to be done for the mechanism of the therapeutic effects on Flavivirus infection.

How did autophagy affect DTMUV replication in spleens or brains? Autophagy has been seen to mediate the innate immunity through the secretion of interferon and inflammation (Deretic et al., 2013). So, we tested the expression levels of innate immune genes in the two tissues with autophagy regulator treatments. There is a limitation of utilizing pharmaceutical autophagy regulators in the research on the interface between autophagy and the immune system. Because chemical inhibitors or inducers always affect multiple cellular pathways, and some chemical regulators can affect immune responses in an autophagicindependent manner (Klionsky et al., 2016). For instance, PI3K is not only the target of many autophagy inhibitors like 3-MA, Wortmannin and LY294002, but also the regulator of Toll-like receptor (TLR)-mediated inflammatory responses (Kuo et al., 2006; Guiducci et al., 2008). 3-MA has been reported to regulate inflammatory response by PI3K-Akt-Glycogen pathway rather than autophagy (Lin et al., 2012). Another type of autophagy inhibitors, including CQ and Bafilomycin A1, are targeting endosomal acidification which is related to the signaling of endosomal TLRs (Rutz et al., 2004; Hart et al., 2005). Actually, genetic approaches and methods based on the specific depletion of ATG proteins from different autophagy functional clusters are the best experimental strategy for this experiment (EchavarriaConsuegra et al., 2019). But it is hard to perform RNA silencing or other protein- or gene-specific targeting technologies in animals of the duck species which is an unconventional animal model. Therefore, to eliminate these problems, we set up both autophagy-enhanced and autophagy-inhibited treatments and then screened out the immune genes whose expression levels varied with autophagy levels. We found that the mRNA levels of RIG-I, MDA5, TLR3, IFN- $\alpha$, IFN- $\beta$, and IL- 8 , were changed with autophagy levels altered by autophagy regulators in the spleen, whereas the mRNA levels of RIG-I, IFN- $\alpha$, and IL-6 in the brain. The expression of type I IFNs is controlled by upstream PRR signaling pathways, including the retinoic RIGI-like receptor (RLR) family and the TLR family (Tian et al., 2019). In the current study, we found that autophagy played a negative role in the expression levels of RLPs and type I IFNs in both duck spleens and brains. HCV-mediated and DENVmediated autophagy also have been reported to suppress RIGI signaling and type I IFN production (Ke and Chen, 2011; Shrivastava et al., 2011). But the mechanism of autophagy suppressed RLR pathways and type I IFNs and the different responses in different organs are still unknown. There was an

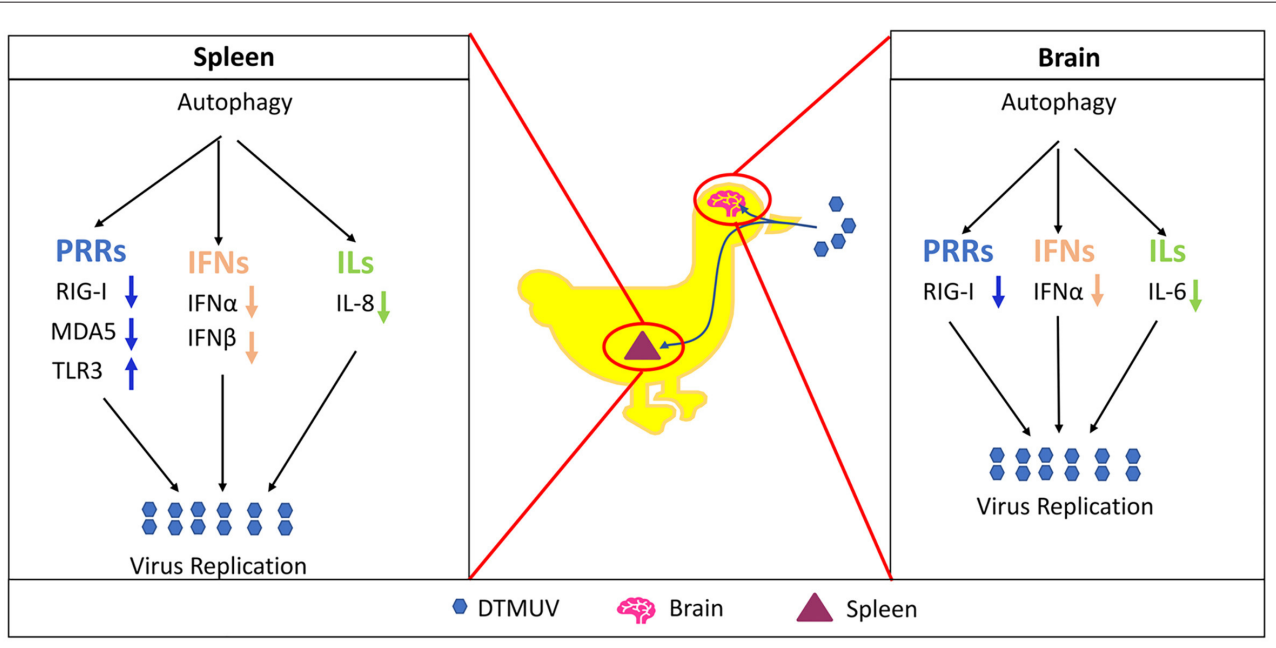

FIGURE 7 | Proposed model of autophagy promotes DTMUV replication and tissue damages in vivo. 
interesting finding that TLR3 levels were negatively correlated with type I IFN levels in the spleens. The signaling of endosomal TLRs might be affected by autophagy-associated membrane fusion events. However, the interaction between autophagy and endosomal TLRs is still unclear and controversial. Autophagy has been reported to be a positive role in TLR7-mediated and TLR9-mediated type I IFN production (Zhou et al., 2012; Hayashi et al., 2018), whereas a negative role in TLR7-mediated type I IFNs in Enterovirus 71 (EV71) and coxsackievirus A16 (CA16) infections (Song et al., 2018). The role of autophagy on TLR3mediated type I IFNs in DTMUV infection needs further study in the future. Cytokines play protective or destructive roles in response to virus infection, and autophagy plays critical roles in virus-mediated inflammation secretion (Zhong et al., 2016; Qian et al., 2017). In the current study, we found autophagy inhibitors enhanced IL-8 mRNA level in the spleen, whereas enhanced IL-6 levels in the brain. Some other reports shows that autophagy inhibition reduces the production of IL-8 (Harris et al., 2011; Luo et al., 2015). We thought this difference might because of the different host species, different pathogens, or different organs. IL- 6 is not only a factor involved in the immune response but also plays many critical roles in the nervous system (Erta et al., 2012). And IL-6 has been reported to be associated with the West Nile virus (WNV)-caused neuroinflammatory (Kumar et al., 2010). The changes of IL-6 level special in the brain might be related to DTMUV-caused neurologic symptoms (Figure 3).

In summary, as shown in Figure 7, the current study demonstrated that DTMUV-trigged autophagy facilitated DTMUV replication, aggravated the developments of pathological symptoms and possibly counteracts the host's innate immunity response in vivo. However, the mechanism of the different autophagy-mediated immune responses in different tissues is still unknown. Above all, our study supports further clinical evidence to use autophagy-related therapies against DTMUV infection and gives some references for the developments of the treatments for other flavivirus infections.

\section{REFERENCES}

Baloch, A. S., Liu, C., Liang, X., Liu, Y., Chen, J., Cao, R., et al. (2019). Avian flavivirus enters BHK-21 cells by a low pH-dependent endosomal pathway. Viruses 11:E1112. doi: 10.3390/v11121112

Blommaart, E. F., Krause, U., Schellens, J. P., Vreeling-Sindelárová, H., and Meijer, A. J. (1997). The phosphatidylinositol 3-kinase inhibitors wortmannin and LY294002 inhibit autophagy in isolated rat hepatocytes. Eur. J. Biochem. 243, 240-246. doi: 10.1111/j.1432-1033.1997.0240a.x

Cao, B., Parnell, L. A., Diamond, M. S., and Mysorekar, I. U. (2017). Inhibition of autophagy limits vertical transmission of Zika virus in pregnant mice. J. Exp. Med. 214, 2303-2313. doi: 10.1084/jem.20170957

Chun, Y., and Kim, J. (2018). Autophagy: an essential degradation program for cellular homeostasis and life. Cells 7:278. doi: 10.3390/cells71 20278

Crazzolara, R., Bradstock, K. F., and Bendall, L. J. (2009). RAD001 (Everolimus) induces autophagy in acute lymphoblastic leukemia. Autophagy 5, 727-728. doi: 10.4161/auto.5.5.8507

Deng, J., Liu, Y., Jia, R., Wang, M., Chen, S., Zhu, D., et al. (2017). Development of an immunochromatographic strip for detection of

\section{DATA AVAILABILITY STATEMENT}

All datasets generated for this study are included in the article/Supplementary Material.

\section{ETHICS STATEMENT}

The animal study was reviewed and approved by the committee of experiment operational guidelines and animal welfare of Sichuan Agricultural University, China (the approved permit number is XF2014-18).

\section{AUTHOR CONTRIBUTIONS}

$\mathrm{ZH}$ and $\mathrm{RJ}$ conceived and designed the experiments. $\mathrm{ZH}$ and $\mathrm{YP}$ guided and conducted the experiment and analyzed the data. $\mathrm{ZH}$ wrote the original draft preparation. RJ and YP reviewed and edited the manuscript. AC, MW, SC, DZ, ML, QY, YW, XZhao, SZ, ZY, YY, LZ, and YL contributed materials. XZhang, JH, SM, XO, BT, LP, and MR helped to analyze the data. All authors read and approved the final manuscript.

\section{FUNDING}

This research was funded by the National Natural Science Foundation of China (31872475), Sichuan Veterinary Medicine and Drug Innovation Group of China Agricultural Research System (SCCXTD-2020-18), China Agricultural Research System (CARS-42-17), Sichuan Province Research Programs (2017JY0014), and the China Scholarship Council for funding Ph.D. fellowships to ZH (No. 201806910033).

\section{SUPPLEMENTARY MATERIAL}

The Supplementary Material for this article can be found online at: https://www.frontiersin.org/articles/10.3389/fcimb. 2020.00155/full\#supplementary-material antibodies against duck Tembusu virus. J. Virol. Methods 249, 137-142. doi: 10.1016/j.jviromet.2017.08.022

Deretic, V., Saitoh, T., and Akira, S. (2013). Autophagy in infection, inflammation and immunity. Nat. Rev. Immunol. 13, 722-737. doi: 10.1038/nri3532

Echavarria-Consuegra, L., Smit, J. M., and Reggiori, F. (2019). Role of autophagy during the replication and pathogenesis of common mosquito-borne flavi-and alphaviruses. Open Biol. 9:190009. doi: 10.1098/rsob.190009

Erta, M., Quintana, A., and Hidalgo, J. (2012). Interleukin-6, a major cytokine in the central nervous system. Int. J. Biol. Sci. 8, 1254-1266. doi: 10.7150/ijbs.4679

Germic, N., Frangez, Z., Yousefi, S., and Simon, H.-U. (2019). Regulation of the innate immune system by autophagy: monocytes, macrophages, dendritic cells and antigen presentation. Cell Death Differ. 26, 715-725. doi: 10.1038/s41418-019-0297-6

Gratton, R., Agrelli, A., Tricarico, P. M., Brandão, L., and Crovella, S. (2019). Autophagy in Zika virus infection: a possible therapeutic target to counteract viral replication. Int. J. Mol. Sci. 20:1048. doi: 10.3390/ijms20051048

Guiducci, C., Ghirelli, C., Marloie-Provost, M.-A., Matray, T., Coffman, R. L., Liu, Y.-J., et al. (2008). PI3K is critical for the nuclear translocation of IRF-7 and type I IFN production by human plasmacytoid predendritic cells in response to TLR activation. J. Exp. Med. 205, 315-322. doi: 10.1084/jem.20070763 
Harris, J., Hartman, M., Roche, C., Zeng, S. G., O'Shea, A., Sharp, F. A., et al. (2011). Autophagy controls IL-1 $\beta$ secretion by targeting pro-IL-1 $\beta$ for degradation. J. Biol. Chem. 286, 9587-9597. doi: 10.1074/jbc.M110.202911

Hart, O. M., Athie-Morales, V., O'Connor, G. M., and Gardiner, C. M. (2005). TLR7/8-mediated activation of human NK cells results in accessory cell-dependent IFN- $\gamma$ production. J. Immunol. 175, 1636-1642. doi: 10.4049/jimmunol.175.3.1636

Hayashi, K., Taura, M., and Iwasaki, A. (2018). The interaction between IKK $\alpha$ and LC3 promotes type I interferon production through the TLR9-containing LAPosome. Sci. Signal. 11:eaan4144. doi: 10.1126/scisignal.aan4144

Hu, Z., Pan, Y., Cheng, A., Zhang, X., Wang, M., Chen, S., et al. (2020). Autophagy promotes duck Tembusu virus replication by suppressing p62/SQSTM1-mediated innate immune responses in vitro. Vaccines 8:E22. doi: $10.3390 /$ vaccines 8010022

Jung, C. H., Ro, S.-H., Cao, J., Otto, N. M., and Kim, D.-H. (2010). mTOR regulation of autophagy. FEBS Lett. 584, 1287-1295. doi: 10.1016/j.febslet.2010.01.017

Ke, P.-Y. (2018). The multifaceted roles of autophagy in flavivirus-host interactions. Int. J. Mol. Sci. 19:E3940. doi: 10.3390/ijms19123940

Ke, P.-Y., and Chen, S. S.-L. (2011). Activation of the unfolded protein response and autophagy after hepatitis $C$ virus infection suppresses innate antiviral immunity in vitro. J. Clin. Invest. 121, 37-56. doi: 10.1172/JCI41474

Klionsky, D. J., Abdelmohsen, K., Abe, A., Abedin, M. J., Abeliovich, H., Acevedo Arozena, A., et al. (2016). Guidelines for the use and interpretation of assays for monitoring autophagy. Autophagy 12, 1-222. doi: 10.1080/15548627.2015.1100356

Klionsky, D. J., and Emr, S. D. (2000). Autophagy as a regulated pathway of cellular degradation. Science 290, 1717-1721. doi: 10.1126/science.290.5497.1717

Kumar, M., Verma, S., and Nerurkar, V. R. (2010). Pro-inflammatory cytokines derived from West Nile virus (WNV)-infected SK-N-SH cells mediate neuroinflammatory markers and neuronal death. J. Neuroinflamm. 7:73. doi: 10.1186/1742-2094-7-73

Kuo, C.-C., Lin, W.-T., Liang, C.-M., and Liang, S.-M. (2006). Class I and III phosphatidylinositol $3^{\prime}$-kinase play distinct roles in TLR signaling pathway. J. Immunol. 176, 5943-5949. doi: 10.4049/jimmunol.176.10.5943

Lee, Y.-R., Hu, H.-Y., Kuo, S.-H., Lei, H.-Y., Lin, Y.-S., Yeh, T.-M., et al. (2013). Dengue virus infection induces autophagy: an in vivo study. J. Biomed. Sci. 20:65. doi: 10.1186/1423-0127-20-65

Lee, Y.-R., Lei, H.-Y., Liu, M.-T., Wang, J.-R., Chen, S.-H., Jiang-Shieh, Y.-F., et al. (2008). Autophagic machinery activated by dengue virus enhances virus replication. Virology 374, 240-248. doi: 10.1016/j.virol.2008.02.016

Li, N., Wang, Y., Li, R., Liu, J., Zhang, J., Cai, Y., et al. (2015). Immune responses of ducks infected with duck Tembusu virus. Front. Microbiol. 6:425. doi: $10.3389 /$ fmicb.2015.00425

Lin, Y.-C., Kuo, H.-C., Wang, J.-S., and Lin, W.-W. (2012). Regulation of inflammatory response by 3 -methyladenine involves the coordinative actions on Akt and glycogen synthase kinase $3 \beta$ rather than autophagy. J. Immunol. 189, 4154-4164. doi: 10.4049/jimmunol.1102739

Liu, Z., Ji, Y., Huang, X., Fu, Y., Wei, J., Cai, X., et al. (2013). An adapted duck Tembusu virus induces systemic infection and mediates antibody-dependent disease severity in mice. Virus Res. 176, 216-222. doi: 10.1016/j.virusres.2013.06.010

Luo, M. X., Wong, S. H., Chan, M. T., Yu, L., Yu, S. S., Wu, F., et al. (2015). Autophagy mediates $\mathrm{HBx}$-induced nuclear factor- $\mathrm{\kappa} \mathrm{B}$ activation and release of IL-6, IL-8, and CXCL2 in hepatocytes. J. Cell. Physiol. 230, 2382-2389. doi: $10.1002 /$ jcp. 24967

Lv, C., Li, R., Liu, X., Li, N., and Liu, S. (2019). Pathogenicity comparison of duck Tembusu virus in different aged Cherry Valley breeding ducks. BMC Vet. Res. 15, 1-9. doi: 10.1186/s12917-019-2020-8

Mateo, R., Nagamine, C. M., Spagnolo, J., Méndez, E., Rahe, M., Gale, M., et al. (2013). Inhibition of cellular autophagy deranges dengue virion maturation. J. Virol. 87, 1312-1321. doi: 10.1128/JVI.02177-12

Mauthe, M., Orhon, I., Rocchi, C., Zhou, X., Luhr, M., Hijlkema, K.-J., et al. (2018). Chloroquine inhibits autophagic flux by decreasing autophagosome-lysosome fusion. Autophagy 14, 1435-1455. doi: 10.1080/15548627.2018.1474314

McLean, J. E., Wudzinska, A., Datan, E., Quaglino, D., and Zakeri, Z. (2011). Flavivirus NS4A-induced autophagy protects cells against death and enhances virus replication. J. Biol. Chem. 286, 22147-22159. doi: 10.1074/jbc.M110.192500

Metz, P., Chiramel, A., Chatel-Chaix, L., Alvisi, G., Bankhead, P., Mora-Rodríguez, R., et al. (2015). Dengue virus inhibition of autophagic flux and dependency of viral replication on proteasomal degradation of the autophagy receptor p62. J. Virol. 89, 8026-8041. doi: 10.1128/JVI.00787-15

Mohl, B.-P., Bartlett, C., Mankouri, J., and Harris, M. (2016). Early events in the generation of autophagosomes are required for the formation of membrane structures involved in hepatitis C virus genome replication. J. Gen.Virol. 97, 680-693. doi: 10.1099/jgv.0.000387

Mustafá, Y. M., Meuren, L. M., Coelho, S. V. A., and de Arruda, L. B. (2019). Pathways exploited by flaviviruses to counteract the blood-brain barrier and invade the central nervous system. Front. Microbiol. 10:525. doi: 10.3389/fmicb.2019.00525

Nikoletopoulou, V., Papandreou, M., and Tavernarakis, N. (2015). Autophagy in the physiology and pathology of the central nervous system. Cell Death Differ. 22, 398-407. doi: 10.1038/cdd.2014.204

Ou, X., Mao, S., Jiang, Y., Zhang, S., Ke, C., Ma, G., et al. (2017). Viralhost interaction in kidney reveals strategies to escape host immunity and persistently shed virus to the urine. Oncotarget 8:7336-7349. doi: 10.18632 /oncotarget.14227

Pei, J., Zhao, M., Ye, Z., Gou, H., Wang, J., Yi, L., et al. (2014). Autophagy enhances the replication of classical swine fever virus in vitro. Autophagy 10, 93-110. doi: 10.4161/auto. 26843

Persaud, M., Martinez-Lopez, A., Buffone, C., Porcelli, S. A., and Diaz-Griffero, F. (2018). Infection by Zika viruses requires the transmembrane protein AXL, endocytosis and low pH. Virology 518, 301-312. doi: 10.1016/j.virol.2018.03.009

Qian, M., Fang, X., and Wang, X. (2017). Autophagy and inflammation. Clin. Transl. Med. 6:24. doi: 10.1186/s40169-017-0154-5

Ren, H., Elgner, F., Jiang, B., Himmelsbach, K., Medvedev, R., Ploen, D., et al. (2016). The autophagosomal SNARE protein syntaxin 17 is an essential factor for the hepatitis C virus life cycle. J. Virol. 90, 5989-6000. doi: 10.1128/JVI.00551-16

Rutz, M., Metzger, J., Gellert, T., Luppa, P., Lipford, G. B., Wagner, H., et al. (2004). Toll-like receptor 9 binds single-stranded CpG-DNA in a sequence-and $\mathrm{pH}-$ dependent manner. Eur. J. Immunol. 34, 2541-2550. doi: 10.1002/eji.200425218

Sánchez-San Martín, C., Liu, C. Y., and Kielian, M. (2009). Dealing with low pH: entry and exit of alphaviruses and flaviviruses. Trends Microbiol. 17, 514-521. doi: 10.1016/j.tim.2009.08.002

Shintani, T., and Klionsky, D. J. (2004). Autophagy in health and disease: a double-edged sword. Science 306, 990-995. doi: 10.1126/science.1099993

Shrivastava, S., Devhare, P., Sujijantarat, N., Steele, R., Kwon, Y.-C., Ray, R., et al. (2016). Knockdown of autophagy inhibits infectious hepatitis $C$ virus release by the exosomal pathway. J. Virol. 90, 1387-1396. doi: 10.1128/JVI.02383-15

Shrivastava, S., Raychoudhuri, A., Steele, R., Ray, R., and Ray, R. B. (2011). Knockdown of autophagy enhances the innate immune response in hepatitis C virus-infected hepatocytes. Hepatology 53, 406-414. doi: 10.1002/hep.24073

Song, J., Hu, Y., Li, J., Zheng, H., Wang, J., Guo, L., et al. (2018). Suppression of the toll-like receptor 7-dependent type I interferon production pathway by autophagy resulting from enterovirus 71 and coxsackievirus A16 infections facilitates their replication. Arch. Virol. 163, 135-144. doi: 10.1007/s00705-017-3592-x

Su, J., Li, S., Hu, X., Yu, X., Wang, Y., Liu, P., et al. (2011). Duck egg-drop syndrome caused by BYD virus, a new Tembusu-related flavivirus. PLOS ONE 6:e18106. doi: 10.1371/journal.pone.0018106

Sun, X., Li, W., Liu, E., Huang, H., Wang, T., Wang, X., et al. (2019a). In vivo cellular and molecular study on duck spleen infected by duck Tembusu virus. Vet. Microbiol. 230, 32-44. doi: 10.1016/j.vetmic.2018.12.003

Sun, X., Liu, E., Iqbal, A., Wang, T., Wang, X., Haseeb, A., et al. (2019b). The dynamic distribution of duck Tembusu virus in the spleen of infected shelducks. BMC Vet. Res. 15:112. doi: 10.1186/s12917-019-1860-6

Sun, Y., Li, C., Shu, Y., Ju, X., Zou, Z., Wang, H., et al. (2012). Inhibition of autophagy ameliorates acute lung injury caused by avian influenza A H5N1 infection. Sci. Signal. 5: ra16-ra16. doi: 10.1126/scisignal.2001931

Sun, Y., Yu, S., Ding, N., Meng, C., Meng, S., Zhang, S., et al. (2014). Autophagy benefits the replication of Newcastle disease virus in chicken cells and tissues. $J$. Virol. 88, 525-537. doi: 10.1128/JVI.01849-13 
Tang, Y., Gao, X., Diao, Y., Feng, Q., Chen, H., Liu, X., et al. (2013). Tembusu virus in human, China. Transbound. Emerg. Dis. 60, 193-196. doi: $10.1111 /$ tbed.12085

Tasaki, T., Nukuzuma, S., and Takegami, T. (2016). Impaired Japanese encephalitis virus replication in p62/SQSTM1 deficient mouse embryonic fibroblasts. Microbiol. Immunol. 60, 708-711. doi: 10.1111/1348-0421.12440

Thontiravong, A., Ninvilai, P., Tunterak, W., Nonthabenjawan, N., Chaiyavong, S., Angkabkingkaew, K., et al. (2015). Tembusu-related flavivirus in ducks, Thailand. Emerg. Infect. Dis. 21, 2164-2167. doi: 10.3201/eid2112.150600

Tian, Y., Wang, M.-L., and Zhao, J. (2019). Crosstalk between autophagy and type I interferon responses in innate antiviral immunity. Viruses 11:132. doi: $10.3390 / \mathrm{v} 11020132$

Tscherne, D. M., Jones, C. T., Evans, M. J., Lindenbach, B. D., McKeating, J. A., and Rice, C. M. (2006). Time-and temperature-dependent activation of hepatitis C virus for low-pH-triggered entry. J. Virol. 80, 1734-1741. doi: 10.1128/JVI.80.4.1734-1741.2006

Wu, Y.-T., Tan, H.-L., Shui, G., Bauvy, C., Huang, Q., Wenk, M. R., et al. (2010). Dual role of 3-methyladenine in modulation of autophagy via different temporal patterns of inhibition on class I and III phosphoinositide 3-kinase. J. Biol. Chem. 285, 10850-10861. doi: 10.1074/jbc.M109.080796

Yamamoto, A., Tagawa, Y., Yoshimori, T., Moriyama, Y., Masaki, R., and Tashiro, Y. (1998). Bafilomycin A1 prevents maturation of autophagic vacuoles by inhibiting fusion between autophagosomes and lysosomes in rat hepatoma cell line, H-4-II-E cells. Cell Struct. Funct. 23, 33-42. doi: 10.1247/ csf.23.33

Yan, P., Zhao, Y., Zhang, X., Xu, D., Dai, X., Teng, Q., et al. (2011). An infectious disease of ducks caused by a newly emerged Tembusu virus strain in mainland China. Virology 417, 1-8. doi: 10.1016/j.virol.2011.06.003

Yu, G., Lin, Y., Tang, Y., and Diao, Y. (2018). Evolution of Tembusu virus in ducks, chickens, geese, sparrows, and mosquitoes in Northern China. Viruses 10:485. doi: $10.3390 / \mathrm{v} 10090485$

Zhang, S., Yi, C., Li, C., Zhang, F., Peng, J., Wang, Q., et al. (2019a). Chloroquine inhibits endosomal viral RNA release and autophagy-dependent viral replication and effectively prevents maternal to fetal transmission of Zika virus. Antiviral Res. 169:104547. doi: 10.1016/j.antiviral.2019.104547

Zhang, X., Jia, R., Pan, Y., Wang, M., Chen, S., Zhu, D., et al. (2019b). Therapeutic effects of duck Tembusu virus capsid protein fused with staphylococcal nuclease protein to target Tembusu infection in vitro. Vet. Microbiol. 235, 295-300. doi: 10.1016/j.vetmic.2019.07.025

Zheng, A., Yuan, F., Kleinfelter, L. M., and Kielian, M. (2014). A toggle switch controls the low $\mathrm{pH}$-triggered rearrangement and maturation of the dengue virus envelope proteins. Nat. Commun. 5:3877. doi: 10.1038/ncomms4877

Zhong, Z., Sanchez-Lopez, E., and Karin, M. (2016). Autophagy, inflammation, and immunity: a troika governing cancer and its treatment. Cell 166, 288-298. doi: 10.1016/j.cell.2016.05.051

Zhou, D., Kang, K. H., and Spector, S. A. (2012). Production of interferon $\alpha$ by human immunodeficiency virus type 1 in human plasmacytoid dendritic cells is dependent on induction of autophagy. J. Infect. Dis. 205, 1258-1267. doi: $10.1093 /$ infdis/jis187

Zhu, K., Huang, J., Jia, R., Zhang, B., Wang, M., Zhu, D., et al. (2015). Identification and molecular characterization of a novel duck Tembusu virus isolate from Southwest China. Arch. Virol. 160, 2781-2790. doi: 10.1007/s00705-015-2513-0

Conflict of Interest: The authors declare that the research was conducted in the absence of any commercial or financial relationships that could be construed as a potential conflict of interest.

Copyright (c) $2020 \mathrm{Hu}$, Pan, Cheng, Zhang, Wang, Chen, Zhu, Liu, Yang, Wu, Zhao, Huang, Zhang, Mao, Ou, Yu, Zhang, Liu, Tian, Pan, Rehman, Yin and Jia. This is an open-access article distributed under the terms of the Creative Commons Attribution License (CC BY). The use, distribution or reproduction in other forums is permitted, provided the original author(s) and the copyright owner(s) are credited and that the original publication in this journal is cited, in accordance with accepted academic practice. No use, distribution or reproduction is permitted which does not comply with these terms. 


\section{OPEN ACCESS}

Edited and reviewed by: Layla Kamareddine,

Qatar University, Qatar

*Correspondence: Anchun Cheng chenganchun@vip.163.com Renyong Jia jiary@sicau.edu.cn

${ }^{\text {t}}$ These authors have contributed equally to this work

Specialty section: This article was submitted to Virus and Host a section of the journal Frontiers in Cellular and Infection Microbiology

Received: 15 December 2020 Accepted: 28 January 2021 Published: 19 February 2021

Citation: Hu Z, Pan Y, Cheng A, Zhang X, Wang $M$, Chen S, Zhu D, Liu M, Yang Q, Wu Y, Zhao X, Huang J,

Zhang S, Mao S, OU X, Yu Y,

Zhang L, Liu Y, Tian B, Pan L, Rehman MU, Yin Z and Jia R (2021)

Corrigendum: Autophagy Is a Potential Therapeutic Target Against Duck Tembusu Virus Infection in Vivo. Front. Cell. Infect. Microbiol. 11:641825. doi: 10.3389/fcimb.2021.641825

\section{Corrigendum: Autophagy Is a Potential Therapeutic Target Against Duck Tembusu Virus Infection in Vivo}

\author{
Zhiqiang $\mathrm{Hu}^{1,2,3+}$, Yuhong Pan ${ }^{1,2,3 t}$, Anchun Cheng ${ }^{1,2,3^{*}}$, Xingcui Zhang ${ }^{1,2,3}$, \\ Mingshu Wang ${ }^{1,2,3}$, Shun Chen ${ }^{1,2,3}$, Dekang Zhu ${ }^{1,2,3}$, Mafeng Liu ${ }^{1,2,3}$, Qiao Yang ${ }^{1,2,3}$, \\ Ying $W u^{1,2,3}$, Xinxin Zhao ${ }^{1,2,3}$, Juan Huang ${ }^{1,2,3}$, Shaqiu Zhang ${ }^{1,2,3}$, Sai Mao ${ }^{1,2,3}$, \\ Xumin Ou ${ }^{1,2,3}$, Yanling $Y u^{1,2,3}$, Ling Zhang ${ }^{1,2,3}$, Yunya Liu ${ }^{1,2,3}$, Bin Tian ${ }^{1,2,3}$, \\ Leichang Pan ${ }^{1,2,3}$, Mujeeb Ur Rehman 1,2,3, Zhongqiong Yin ${ }^{3}$ and Renyong Jia 1,2,3* \\ ${ }^{1}$ Institute of Preventive Veterinary Medicine, Sichuan Agricultural University, Wenjiang, China, ${ }^{2}$ Avian Disease Research \\ Center, College of Veterinary Medicine of Sichuan Agricultural University, Wenjiang, China, ${ }^{3}$ Key Laboratory of Animal \\ Disease and Human Health of Sichuan Province, Sichuan Agricultural University, Wenjiang, China
}

Keywords: DTMUV, autophagy, spleen, brain, tissue damage, replication, immune response

\section{A Corrigendum on}

Autophagy Is a Potential Therapeutic Target Against Duck Tembusu Virus Infection in Vivo By Hu Z, Pan Y, Cheng A, Zhang X, Wang M, Chen S, Zhu D, Liu M, Yang Q, Wu Y, Zhao X, Huang J, Zhang S, Mao S, Ou X, Yu Y, Zhang L, Liu Y, Tian B, Pan L, Rehman MU, Yin Z and Jia R (2020). Front. Cell. Infect. Microbiol. 10:155. doi: 10.3389/fcimb.2020.00155

In the original article, there was a mistake in Figure $\mathbf{3}$ as published. This figure contains the wrong slides of microscopy. The corrected Figure 3 appears below.

The authors apologize for this error and state that this does not change the scientific conclusions in any way. The original article has been updated.

Copyright $\odot 2021 \mathrm{Hu}$, Pan, Cheng, Zhang, Wang, Chen, Zhu, Liu, Yang, Wu, Zhao, Huang, Zhang, Mao, Ou, Yu, Zhang, Liu, Tian, Pan, Rehman, Yin and Jia. This is an open-access article distributed under the terms of the Creative Commons Attribution License (CC BY). The use, distribution or reproduction in other forums is permitted, provided the original author(s) and the copyright owner(s) are credited and that the original publication in this journal is cited, in accordance with accepted academic practice. No use, distribution or reproduction is permitted which does not comply with these terms. 
A

Saline

DTMUV+Saline

DTMUV+Rapa

DTMUV+3-MA

DTMUV $+C Q$
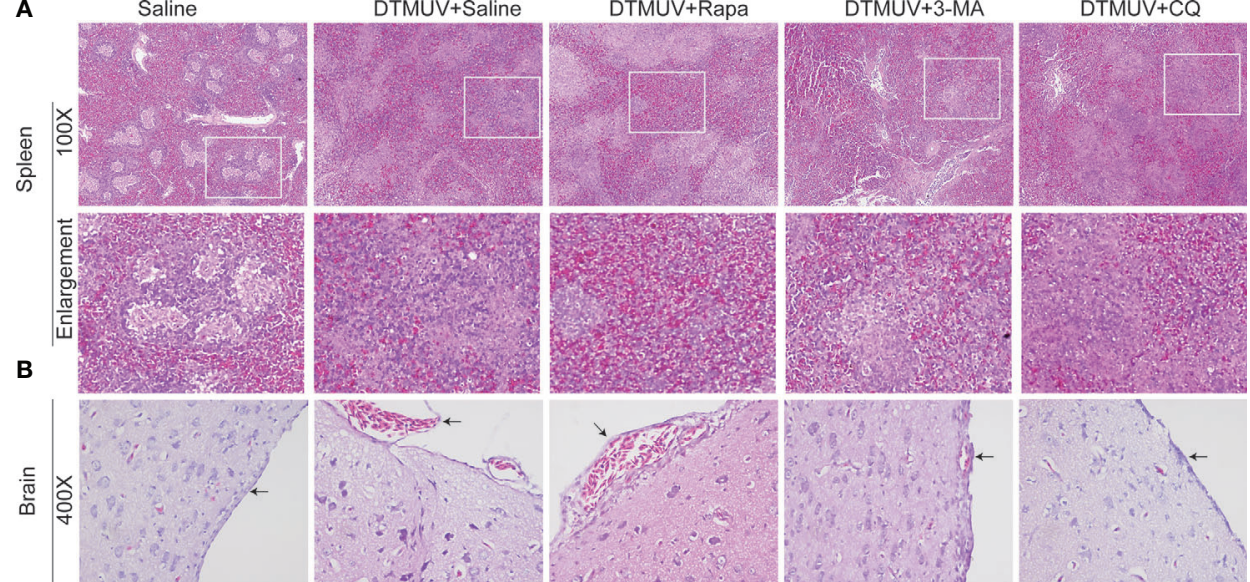

FIGURE 3 | Hematoxylin and eosin staining of cells in the spleens (A) and the brains (B) of ducks infected with DTMUV in the absence or presence of either Rapa, 3-MA, or CQ, respectively. Treatment with saline was used as the control. Images shown were representative from five ducks in each group. The images were with 100X magnification for spleens and 400X for brains. Black arrows: blood cells in the meninx. 


\title{
Drosophila as a Model Organism in Host-Pathogen Interaction Studies
}

\author{
Salma Younes ${ }^{\dagger}$, Asma Al-Sulaiti ${ }^{\dagger}$, Elham Abdulwahab Ahmed Nasser ${ }^{\dagger}$, Hoda Najjar and \\ Layla Kamareddine*
}

Biomedical Sciences Department, College of Health Sciences, QU Health, Qatar University, Doha, Qatar

\section{OPEN ACCESS}

Edited by:

Eric Ghigo,

IHU Mediterranee Infection, France

Reviewed by:

Thomas Roeder,

University of Kiel, Germany

Shruti Yadav,

Molecular Medicine Research

Institute, United States

*Correspondence:

Layla Kamareddine

Ikamareddine@qu.edu.qa

†These authors have contributed equally to this work

Specialty section:

This article was submitted to

Bacteria and Host,

a section of the journal

Frontiers in Cellular and Infection

Microbiology

Received: 12 February 2020 Accepted: 20 April 2020

Published: 23 June 2020

Citation:

Younes S, Al-Sulaiti A, Nasser EAA, Najjar $H$ and Kamareddine $L$ (2020) Drosophila as a Model Organism in Host-Pathogen Interaction Studies. Front. Cell. Infect. Microbiol. 10:214.

doi: 10.3389/fcimb.2020.00214
Owing to the genetic similarities and conserved pathways between a fruit fly and mammals, the use of the Drosophila model as a platform to unveil novel mechanisms of infection and disease progression has been justified and widely instigated. Gaining proper insight into host-pathogen interactions and identifying chief factors involved in host defense and pathogen virulence in Drosophila serves as a foundation to establish novel strategies for infectious disease prevention and control in higher organisms, including humans.

Keywords: Drosophila, host-pathogen interactions, host defense factors, pathogen virulence factors, disease control, disease progression

\section{INTRODUCTION}

Drosophila, a chief tool in contemporary genetic studies, became one of the most powerful model organisms widely used in scientific explorations. The versatility, low cost, short life cycle, well-characterized genome, and feasibility of genetic manipulation made the fruit fly an indispensable model organism for basic research. The modern era of Drosophila research initially took off when the fly was deployed in developmental biology, particularly in fly embryo studies to identify novel genes involved in development (Nusslein-Volhard and Wieschaus, 1980). Further studies conducted in Drosophila have contributed to novel groundbreaking findings that allowed the identification of fundamental components of different pathways conserved between the fruit fly and higher mammalian organisms, including humans. Recently, Drosophila gained great popularity in host-pathogen interaction and infectious disease control studies due to several reasons, many of which were attributed to evolutionary conserved features in both Drosophila and vertebrates including innate immune cascades, signal transduction pathways, and transcriptional regulators. The fruit fly surprisingly serves as a host for a diversity of pathogens and could be readily infected with these pathogens naturally or in an experimental setting. The existence of a wide array of molecular and genetic tools that allow gene manipulation in specific cells/tissues in the fly also favors its use in host-pathogen interaction studies. Genetic and genome-wide RNAi screens in either intact flies or cell lines have identified a wide array of host effector molecules and pathways involved in host defense against invading pathogens. Reciprocally, flies can be used to screen for pathogen-virulence factors. The fly's GAL4-UAS transactivation system (Brand and Perrimon, 1993) allows the direct expression of transgenes encoding host or pathogen proteins in a cell-type-specific manner in vivo. Also, the fly's LexA transcriptional system, allows combinatorial gene expression in a distinct or overlapping fashion in vivo (Pfeiffer et al., 2010; Yagi et al., 2010), opening up for the feasibility of conducting epistasis analysis and revealing a role of specific genes in regulating cellular processes and pathways. Such experiments are difficult to be conducted in higher model organisms including mammals, advocating the use of Drosophila in host-pathogen interaction studies. Like all invertebrates, Drosophila lacks an adaptive immune response and relies exclusively 
on innate immunity with both its humoral and cellular arms to fight off invading pathogens. These innate immune responses mainly include production of antimicrobial peptides (AMPs) and anti-pathogenic factors through core signaling pathways (Toll, IMD, and JAK/STAT), anti-viral response through the RNA interference (RNAi) pathway, and pathogen immobilization through phagocytosis, encapsulation, and melanization (Agaisse and Perrimon, 2004; Akira et al., 2006; Govind, 2008). In this review, we provide an overview of the use of Drosophila in host-pathogen interaction studies and highlight the role of the fly's innate immune system in pathogen control. We also recapitulate a broad spectrum of host defense and pathogen virulence factors identified in Drosophila-pathogen studies and involved in microbial control and disease progression.

\section{HOST DEFENSE FACTORS}

Drosophila is considered a significant model organism in studying host-pathogen interactions (Figure 1). The establishment of the D. melanogaster whole genome sequence in 2000 (Adams et al., 2000) paved the way for adapting existing high-throughput RNAi screening methodologies in Drosophila cell lines to study gene function and identify specific gene targets and immune-associated components and modulators (Ueda, 2001; Kiger et al., 2003). Combining the findings of high-throughput RNAi screens with classical genetic methods and in vivo fly studies enabled the identification of humoral and cell-mediated host defense factors against a wide array of intracellular and extracellular pathogens (Cherry, 2008; Bier and Guichard, 2012).

\section{Humoral Host Defense}

Humoral innate immune responses in Drosophila mainly include production of AMPs and anti-pathogenic factors through Toll, IMD, and JAK/STAT signaling pathways. The primarily role attributed to the Toll pathway was its involvement in Drosophila embryonic development (Nusslein-Volhard and Wieschaus, 1980). In 1995, Hultmark et al. introduced Toll (Toll-1) as a potent immune activator in fruit fly cell lines (Rosetto et al., 1995). Since then, the Toll pathway was shown to be implicated in immune defense against an array of pathogens. Unlike the mammalian Toll pathway, the activation of Drosophila Toll signaling is not initiated by direct interaction with microbial determinants, but rather by the cleaved form of spätzle, a cytokine-like molecule that is thought to be processed by secreted serine proteases (SPs) and spätzle-processing enzyme (SPE). SPs and SPE are regulated by several pathogen recognition receptors (PRRs) including peptidoglycan recognition protein SA (PGRP-SA), PGRP-SD, Gram-negative binding protein 1 (GNBP1), and GNBP3 (Gottar et al., 2006). To avoid exaggerated immunity, the activation of the Toll pathway is generally tightly regulated. Upregulation of Spn1, a member of the serpin superfamily protease inhibitors located upstream of SPE, for example, contributes to the Toll pathway inactivation and to a downregulation in the expression of AMPs, mainly Drosomycin. Fungal-infected Spn1 null mutants exhibit an up-regulation in Drosomycin (Fullaondo et al., 2011). ModSP, a modular serine protease, activates the Toll pathway to culminate in AMP production. ModSP mutant flies challenged with either grampositive bacteria (Enterococcus faecalis or Listeria monocytogenes) or fungal species (Candida albicans) succumb to death-associated reduction in AMP gene expression (Buchon et al., 2009a). In addition to its well-defined role against fungal and gram-positive bacteria, Oh et al. reported a role of the Toll pathway in defense against acid-fast mycobacteria. Mycobacterium abscessus, a non-tuberculous mycobacteria in humans, colonizes the gut of $D$. melanogaster and induces predominant expression of Drosomycin upon Toll pathway activation (Oh et al., 2013). Strikingly, Gottar et al. identified a pathway that acts jointly with GNBP3 to activate the Toll pathway upon fungal infection. PR1, a C. albicans virulence factor, activates Toll signaling by promoting the proteolytic cleavage and maturation of the Persephone protease (PSH). This finding indicates that the detection of fungal infection in Drosophila is dependent on both the recognition of foreign fungal invariant patterns and on tracking the consequence of virulence elements on the infected host (Gottar et al., 2006). Interestingly, and although both GNBP3 and PSH-dependent pathway are also required for Toll pathway activation upon Candida glabrata infection, only GNBP3 mutants are susceptible to Candida glabrata infection, implicating that the downstream effector mechanisms like AMP production and melanization activated against different fungal infections may not be the same (Chamilos et al., 2010; Quintin et al., 2013). Several studies have also employed D. melanogaster as a model organism to characterize anti-viral Toll immunity. The Toll pathway was shown to play a role in efficiently inhibiting Drosophila X viral (DXV) replication. Interestingly, the levels of Drosophila AMP genes induced in response to DXV infection were similar to those reported during Escherichia coli infection (Zambon et al., 2005). Extracellular virions, which were first discovered in Drosophila, and currently in metazoans, are also recognized by Toll-like receptors located on cell surfaces and inside endo-lysosomal compartments (Medzhitov, 2001).

Recently, the impact of post-translational modifications on modulating Toll signaling has been also studied in fruit flies. Such modifications were shown to change the localization and trafficking of the protein in a cell, enhance or inhibit the protein activity, and/or alter the protein's ability to bind to protein signaling partners. The Drosophila Ubc9/Lwr enzyme, for example, affects Toll signaling by stimulating the sumoylation of the Dorsal transcription factor (Schmidt, 2014). Likewise, $\beta$-arrestin Kurtz (Krz) regulates Toll signaling via protein sumoylation by interacting with the SUMO protease Ulp1. $\mathrm{Krz}$ or Ulp1 Drosophila larval mutants exhibit inflammationlike phenotypes characterized by elevation in lamellocyte production, formation of melanotic tumors, accumulation of transcriptional effectors (Dorsal and Dif) of the Toll pathway, and increased expression of anti-microbial peptides (Drosomycin). Interestingly, loss of function of these two genes reveal a dose-dependent sensitive and synergistic response, suggesting that they belong to the same signaling pathway (Anjum et al., 2013). Moreover, Pellinos, a family of E3 ubiquitin ligases, were shown to also regulate Toll signaling by catalyzing 


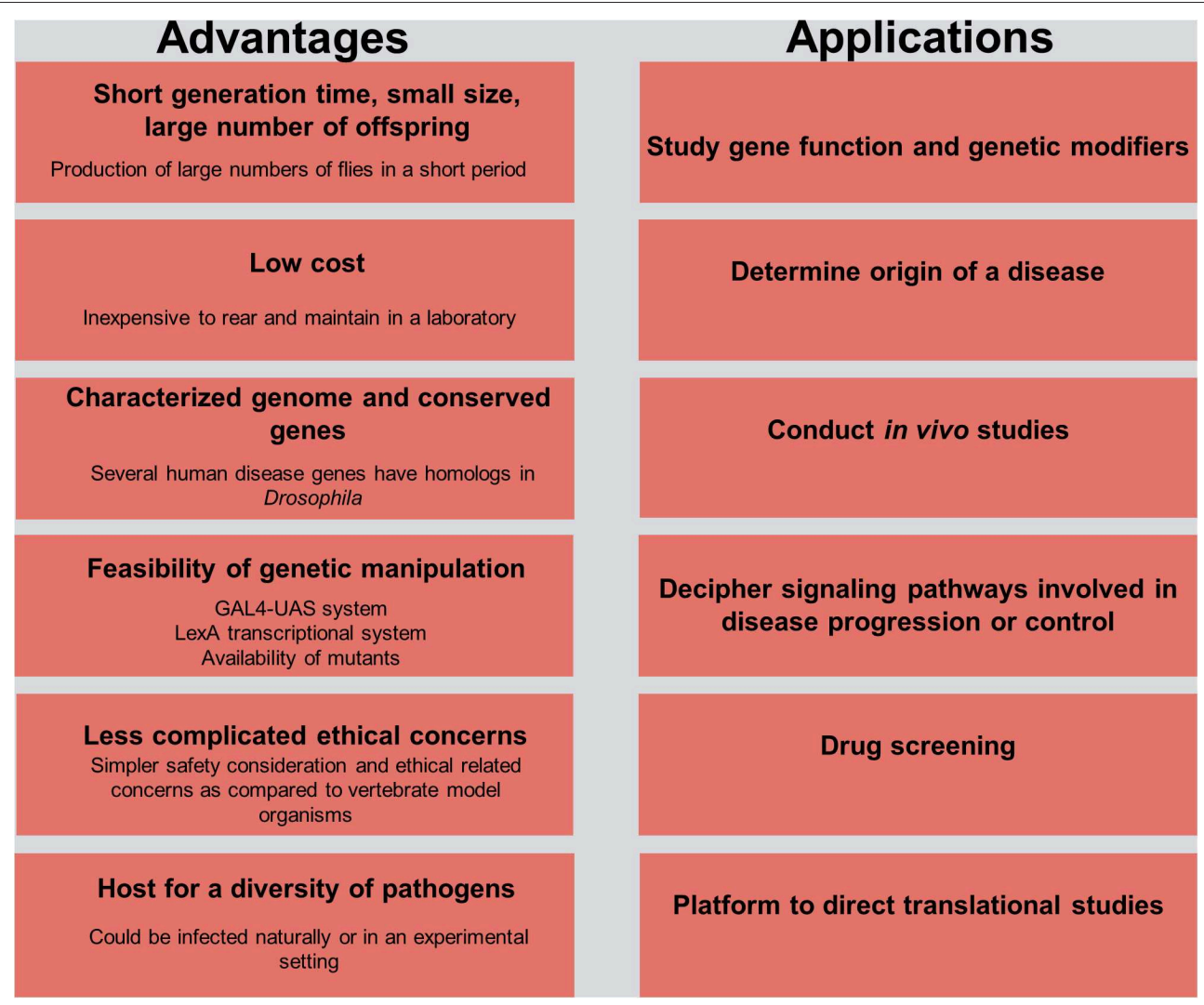

FIGURE 1 | Advantages and practical applications in Drosophila for host-pathogen interaction studies. The left side of the figure delineates the advantages of using the fruit fly model organism in research, and the right side outlines its use as a platform for understanding the etiology of a disease and the potential means of controlling it.

the K63-linked polyubiquitination of Pelle, an IL-1 receptorassociated kinase homolog in Drosophila (Medvedev et al., 2015). Genome-wide screening studies of the Toll pathway also identified novel immune-associated components and regulators including the Deformed Epidermal Auto-regulatory Factor 1 (DEAF1) transcription factor as an essential component for the expression of the Toll target AMP Drosomycin (Kuttenkeuler et al., 2010).

Similar to the Toll pathway, the Drosophila IMD pathway, which is mainly directed against gram-negative pathogens, plays a fundamental role in humoral immunity through AMP production and pathogen clearance. IMD mutant flies, for example, are sensitive to Vibrio cholerae infection (Wang et al., 2013; Kamareddine et al., 2018a), while those with a gain-offunction mutation exhibit resistance, plausibly by lowering the virulence effect of the cholera toxin via increasing the rate of intestinal stem cell division (Wang et al., 2013). Dredd ${ }^{D 55}$ IMD mutant flies infected with Xenorhabdus nematophila and Photorhabdus luminescens nemato-bacterial composites also fail to survive infection compared to Dif ${ }^{1}$ Toll mutants and wild-type infected flies, albeit the $24 \mathrm{~h}$ priming with non-pathogenic $E$. coli prior to $X$. nematophila and $P$. luminescens infection. These findings advocate the notion that $X$. nematophila and $P$. luminescens pathogens target components of the IMD pathway, despite AMPs synthesis triggered by the nemato-bacterial composite infection (Aymeric et al., 2010). Apart from its well-defined role against gram-negative bacteria, recent studies have also highlighted a role of IMD signaling in defense against fungal and gram-positive bacterial infection (De Gregorio et al., 2002a; Hedengren-Olcott et al., 2004; Pham et al., 2007; Dionne and Schneider, 2008; Costa et al., 2009). Interestingly, non-canonical AMP-independent IMD immunity have been also shown to be crucial in the Drosophila gut defense system. Hori et al. reported that IMD mutant flies are sucseptible to Staphylococcus aureus oral infections and revealed a role of the IMD pathway in clearance of $S$. aureus from the fly gut (Hori et al., 2018). In alliance with this distinctive role in gut immunity, the IMD pathway was also shown to control gut homeostatic balance in a microbiota-dependent-infectionindependent context. The gut flora, which induces IMD signaling activation, significantly affects the midgut transcriptome and promotes the expression of key genes involved in host physiology. A study by Kamareddine et al. revealed that IMD signaling in enteroendocrine cells activated by the intestinal microbiota acetate metabolite regulates the expression of the tachykinin peptide hormone, promoting metabolic homeostasis in the host. Both germ-free flies and IMD mutant flies were shown to behave similarly by exhibiting developmental retardation, disrupted lipid metabolism, and a status of inactive insulin signaling (Kamareddine et al., 2018a). Owing to the fact that 
humoral immunity in Drosophila is chiefly mediated by AMP production by fat body cells, particular attention has been also given to our understanding of IMD signaling in the fat body. A study by Tsichritzis et al. (2007) revealed that the deubiquitinase Cylindromatosis (CYLD) inhibits NF- $\mathrm{B}$ signaling and downregulates the IMD response. Although CYLD mutant flies exhibit an increase in AMP expression, particularly those with prior infections, yet these mutants succumb to death significantly faster than controls upon E. coli infection. Although the target of CYLD in the IMD pathway remains uncharacterized, this poor survival rate of CYLD-deficient flies could be attributed to an alteration in the structure and function of fat body cells, as CYLD regulates homeostatic balance in these cells. Interestingly, several factors that affect physiology and development in a host also affect Toll and IMD signaling through manipulating fat body maturation. The induction of Diptericin expression in larvae, for example, is affected by age and is dependent on the presence of the ecdysone molting hormone. A mutation affecting the metabolism of ecdysone could indirectly affect the immune status of a host (Meister and Richards, 1996; Ligoxygakis et al., 2002a). It is worth noting here that signaling mechanisms between the gut and the fat body contribute to the regulation of systemic immune responses in the host (Lemaitre and Hoffmann, 2007). Upon oral infection, Ecc15 and P. entomophila can colonize and multiply in the fly gut, triggering strong systemic immunity, without a need for those bacterial species to cross the wall of the gut (Vodovar et al., 2005; Acosta Muniz et al., 2007).

Since the IMD pathway is similar to the mammalian tumor necrosis factor receptor (TNFR) pathway (Leulier et al., 2000; Costa et al., 2009), which plays a critical role in infectious disease control particularly against viral infections (Herbein and O'Brien, 2000), several studies deployed Drosophila as a model organism to gain further insight into the role of IMD signaling in anti-viral immunity. The cricket paralysis virus $(C r P V)$, an RNA virus that infects a wide range of insect hosts, displays increased virulence with higher viral loads in IMD mutant flies (Costa et al., 2009). Interestingly, IMD signaling-mediated anti-CrPV immunity seems to be also AMP independent. Similar to $C r P V$ infection, sindbis viral replication increases in IMD mutant flies (Avadhanula et al., 2009). Moreover, knocking down the peptidoglycan recognition protein-LC (PGRP-LC), a membrane associated IMD pathway receptor, in Drosophila S2 cells also promotes an increase in the genome copy number of the sigma virus and causes a significant up-regulation in the expression of the $L$ gene compared to other viral genes (Liao et al., 2019). An anti-microbial RNAi signaling screen performed by Foley et al. publicized different classes of negative and positive gene regulators of IMD signaling including those that enhance response to peptidoglycan stimulation (46 EDRi genes), and others that constitutively activate NF-kB in the absence of LPS induction (26 CDRi genes) (Blandin et al., 2004). Further screens identified additional IMD positive regulators including Iap2 and TAB (Garver et al., 2006; Kawai and Akira, 2006). Similar to the studies that have been conducted on the Toll pathway, the impact of post-translational modifications on regulating IMD signaling has been also recently deliberated in fruit flies. SP36/Scny was shown to negatively regulate IMD signaling transduction by hydrolyzing UbK63, a key player in IMD ubiquitination (Thevenon et al., 2009). Similarly, USPs were also shown to regulate IMD immune signaling. USP2, for instance, deubiquitinates Imd, promoting its degradation (Engel et al., 2014).

The JAK/STAT signaling pathway, which controls various biological processes and tissue hemostasis in both mammals and invertebrates, also contributes to humoral immunity in a host. It is mainly activated upon microbial infection and/or cellular damage induced by stress response/pathogen infection, and culminates in the production of regulatory molecules, antiviral agents, and anti-bacterial agents including AMPs. Cell damage induced by Serratia marcescens and Erwinia carotovora infection in Drosophila for example induces JAK/STAT signaling (Buchon et al., 2009b; Cronin et al., 2009) and activates a gutspecific defense machinery characterized by the expression of a subset of AMPs including the Drosomycin-like peptide (dro3). This activation, which is pathogen specific and triggered by cell damage caused by bacterial infection rather than by the bacteria itself (Buchon et al., 2009c), is particularly important in maintaining gut homeostasis by controlling epithelial cell proliferation and renewal in response to bacterial infection (Buchon et al., 2009b; Jiang et al., 2009). In the absence of infection, the indigenous gut flora triggers the expression of $h o p^{\text {Tum-l }}$ or $u p d 3$, which is generally adequate to induce intestinal stem cell progeny differentiation and gut regeneration through JAK/STAT and JNK signaling (Buchon et al., 2009b). Moreover, global gene expression analysis of Drosophila gut tissues to oral Erwinia carotovora infection revealed an important contribution of IMD and JAK/STAT pathways, but not the Toll pathway, to the regulation of gut immune responses (Buchon et al., 2009c). Although the intricate contribution of JAK/STAT signaling to cellular immunity has not been fully understood, it has been thought to be involved in cellular responses like hemocyte proliferation and differentiation (Agaisse and Perrimon, 2004). Recently, Yang et al., reported a role of JAK/STAT signaling in parasitoid egg wasp encapsulation in infected Drosophila larvae (Yang et al., 2015). Several studies addressing the role of JAK/STAT pathway in anti-viral immunity also revealed that the expression of "traditional" JAK/STAT pathway target genes including $u p d 2$, upd3, and TotM, is induced by many viral species including vesicular stomatitis virus, Flock House virus, and Drosophila X virus (Kemp et al., 2013; Myllymaki and Ramet, 2014). Likewise, the Drosophila $\mathrm{C}$ virus infection triggers the expression of several genes like virus-induced RNA-1 (vir-1). Many of these induced genes enclose STAT binding sites in their promoter regions, and their activation is therefore dependent on JAK/STAT signaling. The JAK tyrosine kinase Hopscotch (Hop) was also shown to be involved in controlling Drosophila C virus loads and to participate in inducing the expression of some virus-regulated genes. Deficiencies in JAK/STAT signaling increases Drosophila $\mathrm{C}$ virus load and exhibits high mortality rates in infected flies (Dostert et al., 2005). Although double-stranded RNA (dsRNA) itself does not induce viral response in Drosophila, recent studies have shown that recognizing virus-derived dsRNA 
through the amino terminal $\mathrm{DExD} / \mathrm{H}$-box helicase domain of Dicer-2 promotes the expression of the vago-secreted protein (Paradkar et al., 2012) that plays an antiviral role against Drosophila C virus infection (Paradkar et al., 2012). Interestingly, vago seems to induce the JAK/STAT pathway through a Dome-independent mechanism, signifying the existence of an alternative receptor that is yet to be determined. This finding provides a conceivable role of vago in connecting both RNAi and JAK/STAT signaling pathways, suggesting that vago, which is thought to be insect specific, could serve as a cytokine and functionally relate to the mammalian interferon system (Paradkar et al., 2012). By comparing RNA interference (detailed in the section below) with JAK/STAT anti-viral immunity, however, RNAi interference epitomizes an effectual antiviral machinery that operates against an array of RNA and DNA viruses, unlike the antiviral contribution of JAK/STAT signaling, which seems to be more species specific (Kemp et al., 2013) (Figure 2).

\section{Cell-Mediated Host Defense}

Phagocytosis, which is involved in ingesting apoptotic debris and destroying foreign pathogens by hemocytes (plasmatocytes, crystal cells, and lamellocytes), represents a fundamental mean of maintaining tissue homeostasis (Lemaitre and Hoffmann, 2007). Various receptors and key players chiefly involved in the phagocytic process have been identified in Drosophila. Pearson et al. revealed that the Drosophila scavenger receptor C1 (SR$\mathrm{CI}$ ), which has a broad polyanionic ligand-binding specificity similar to the mammalian class A macrophage-specific scavenger receptor (SR-A), exhibits great affinity and saturable binding of ${ }^{125}$ I-labeled acetylated low-density lipoprotein when expressed in mammalian cells (Pearson et al., 1995). Cuttell, et al. highlighted a previously uncharacterized role of the CED1/6/7 pathway in phagocytosis, by demonstrating that Draper (a CED-1homolog that belongs to the CED1/6/7 pathway)-mediated phagocytosis requires the Drosophila Junctophilin protein, Undertaker (UTA), and is linked to $\mathrm{Ca}^{2+}$ homeostasis (Cuttell et al., 2008). Additionally, Kocks et al. identified a role of Eater, an EGF-like repeat transmembrane receptor of the Nimrod family present on Drosophila hemocytes, in bacterial phagocytosis (Kocks et al., 2005). Likewise, Bretscher et al., uncovered the contribution of Eater in hemocyte localization, attachment, and adhesion, and in efficient phagocytosis of gram-positive (Staphylococcus aureus, Staphylococcus epidermidis, Micrococcus luteus), but not gram-negative (Escherichia coli and Serratia marcescens) bacteria (Bretscher et al., 2015). The intergin $\beta v$ phagocytic receptor was also shown to be involved in defense against septic but not oral S. aureus infection in Drososphila (Shiratsuchi et al., 2012). Studies in Drosophila S2 cells in turn identified a role of PGRP-LC in phagocytosis of gram-negative (E. coli), but not gram-positive bacteria (Ramet et al., 2002). Apart from its scavenger function, PGRP-SC1 was also shown to act as an opsonin, and therefore, contribute to bacterial phagocytosis (Garver et al., 2006). Several thioester proteins (TEPs) identified in different insect species including Anopheles gambiae, also act as a bona fide opsonin to promote gram-positive and gram-negative bacterial phagocytosis (Levashina et al., 2001).
In Drosophila, functional data publicized a role of several fruit fly TEPs, including TEP2, TEP3, and TEP6 in binding to several pathogens including E. coli, S. aureus, and C. albicans, respectively (Stroschein-Stevenson et al., 2006). Interestingly, Croquemort (CRQ), a CD36-related receptor that is exclusively expressed on macrophages in Drosophila embryo, was shown to be required for effectual phagocytosis of apoptotic corpses, but is not necessary for bacterial engulfment (Franc et al., 1999). Several screens identified cellular mediators of phagocytosis. Among those genes are four transcription factors, one of which encodes the GATA-factor Serpent, a chief regulator of hematopoesis in flies. Complimentary expression profile studies identified 45 genes, including the SR-C1 scavenger receptor gene that is down-regulated upon Serpent depletion (Meister and Tuschl, 2004; Haasnoot and Berkhout, 2006). RNAi against these Serpent-dependent genes further identified Eater and Nimrod phagocytic receptors (Miyano-Kurosaki and Takaku, 2006). Given that various classes of entry receptors plausibly facilitate the uptake of different microbes, although overlying and repetitive specificities do exist occasionally, many screen studies are usually done following specific microbial infections. Stroschein-Stevenson et al. identified 184 genes essential for efficient fungal uptake using Candida-infected phagocytic S2 cells. Among those genes is the macroglobulin-related protein (Mcr), which specifically opsonize Candida, unlike TEP2 and TEP3 that are needed for opsonization and efficient uptake of E. coli and S. aureus, respectively (Stroschein-Stevenson et al., 2006). Another screen following Mycobacteria fortuitum infection revealed 54 genes including the novel class B scavenger receptor peste (Li et al., 2002) that is essential for Mycobacteria fortuitum and Listeria monocytogenes, but not for E. coli and S. aureus uptake in S2 cells (Galiana-Arnoux et al., 2006). Genomewide RNA interference screening was put forth to introduce host factors that block intracellular bacterial pathogenesis using cells. Interestingly, comparative studies of host defense genes involved in hindering bacterial pathogenesis revealed that some host factors have general inhibitory roles in intracellular pathogenesis, while others specifically affect the mechanistic ability of certain bacterial species to access the host (Agaisse et al., 2005). Rab7, CG8743, and the ESCRT machinery, for instance, represent unique vulnerability factors of the host cell, as manipulating any of these factors alone no longer constrains the growth of the non-pathogen Mycobacterium smegmatis in Drosophila (Yang et al., 2015). A similar study on Mycobacterium marinum also identified the lysosomal enzyme beta-hexosaminidase as an imperative factor in modulating mycobacterial growth. Remarkably, this bactericidal activity of $\beta$-hexosaminidase seems to be Mycobacterium marinum specific, as it is not involved in constraining the growth of other bacterial species like Salmonella typhimurium and Listeria monocytogenes (Koo et al., 2008).

Encapsulation is a another cellular response that is devoted to eliminate pathogens by forming hemocytic capsules around foreign bodies that are outsized to be phagocytozed (Kounatidis and Ligoxygakis, 2012). In Drosophila, cellular encapsulation happens in three stages. During the first stage, hemocytes, plausibly through their surface receptors, primarily recognize the 


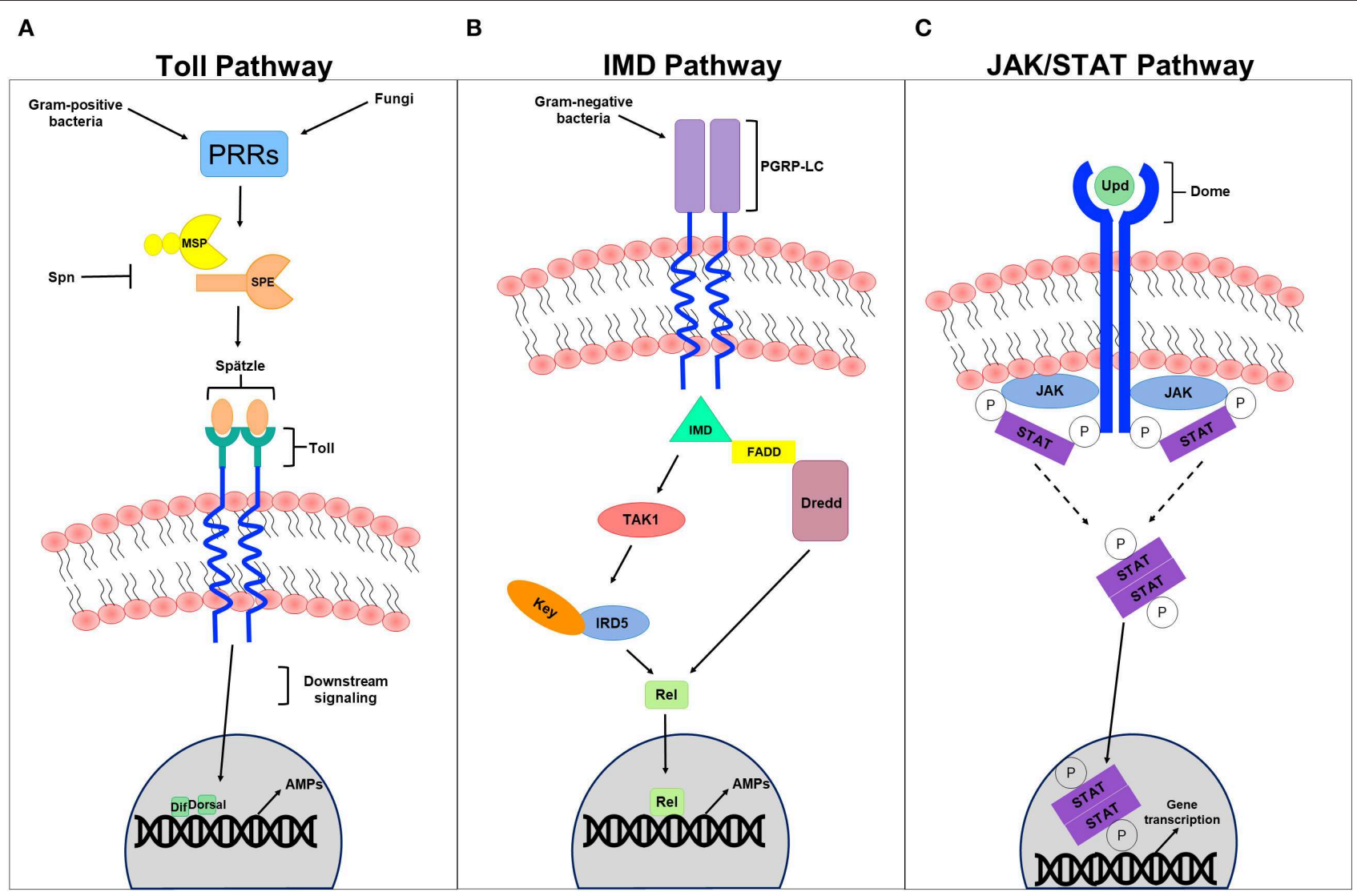

FIGURE 2 | Humoral innate immune signaling pathways. (A) Represents a schematic diagram of the Toll pathway. Gram-positive bacteria and fungi recognized by pathogen recognition receptors (PRRs) trigger the activation of this pathway. Modular serine protease (MSP) and spätzle-processing enzyme (SPE), which are regulated by several PRRs are thought to process the cleavage of the spätzle ligand into a mature spätzle that binds to the Toll receptor, initiating downstream signaling pathway that culminates in the translocation of the NFB-like transcription factors Dif and/or Dorsal into the nucleus, promoting the expression to antimicrobial peptides (AMPs) in response to infection. Serpin (Spn) tightly regulates the primary steps of this pathway to avoid exaggerated immunity. (B) Represents a schematic diagram of the IMD pathway. Gram-negative bacteria recognized by receptors of the IMD pathways like the peptidoglycan recognition protein-LC (PGRP-LC) trigger the pathway activation, promoting the formation of the IMD, FADD, and Dredd (caspase 8 homolog) complex. This in turn activates Dredd, which is thought to be involved in the cleavage of the NFB-like transcription factors Relish (Rel). This formed complex also activates Tak1 (MAP3 kinase) and the IKK complex (IRD5 and key) to phosphorylate Rel. Once translocated into the nucleus, Rel promotes the expression to AMPs in response to invading pathogens. (C) Represents a schematic diagram of the JAK/STAT pathway. The UPD ligand binds to the DOME receptor leading to its activation. The phosphorylation of JAK and DOME create docking puts for STATs recruited to the formed complex. STATs themselves become phosphorylated generating an active dimer that translocates to the nucleus, promoting effector gene expression.

parasitoid egg as a non-self. This recognition further promotes changes in the hemocyte cell surface membrane, exposing hidden molecules and presumably triggering downstream signaling (Nappi et al., 1991, 2000). During the second stage of encapsulation, the number of circulating hemocytes increases for a short term, and lamellocytes differentiate from plasmatocytes (Rizki and Rizki, 1990). Plasmatocytes account for more than $90 \%$ of all mature larval hemocytes and are involved in the phagocytic elimination of pathogenic microorganisms and dead cells (Lemaitre and Hoffmann, 2007). Activated lamellocytes, which are only present in larvae and whose expression is mainly induced upon infection, traffic to the parasitoid egg, flatten, and attach to the egg and to each other, creating a multilayered capsule (Strand and Pech, 1995). Lamellocytes are particularly involved in encapsulating and neutralizing invading pathogens that are too large to be up-taken by phagocytosis (Lemaitre and Hoffmann, 2007). The third stage of encapsulation involves crystal cells that account for $5 \%$ of the larval hemocytes. These cells function as storage sites for pro-phenoloxidase (PPO) and are therefore involved in the melanotic defensive response. Lysis of crystal cells triggers the melanization of the capsule surface (Strand and Pech, 1995; Fellowes and Godfray, 2000; Lemaitre and Hoffmann, 2007). Within the capsule, the encapsulated parasitoid egg gets killed by either direct asphyxiation (Salt, 1970) or by the release of superoxide anions or hydroxyl radicals from the capsule content (Nappi et al., 1995, 2000; Nappi and Vass, 1998) (Figure 3).

Melanization is another prominent immune response in insects characterized by melanin synthesis and deposition around intruding microorganisms (Christensen et al., 2005). Melanization is also involved in wound healing, phagocytosis, blood coagulation, and AMP expression in arthropods (Ashida 


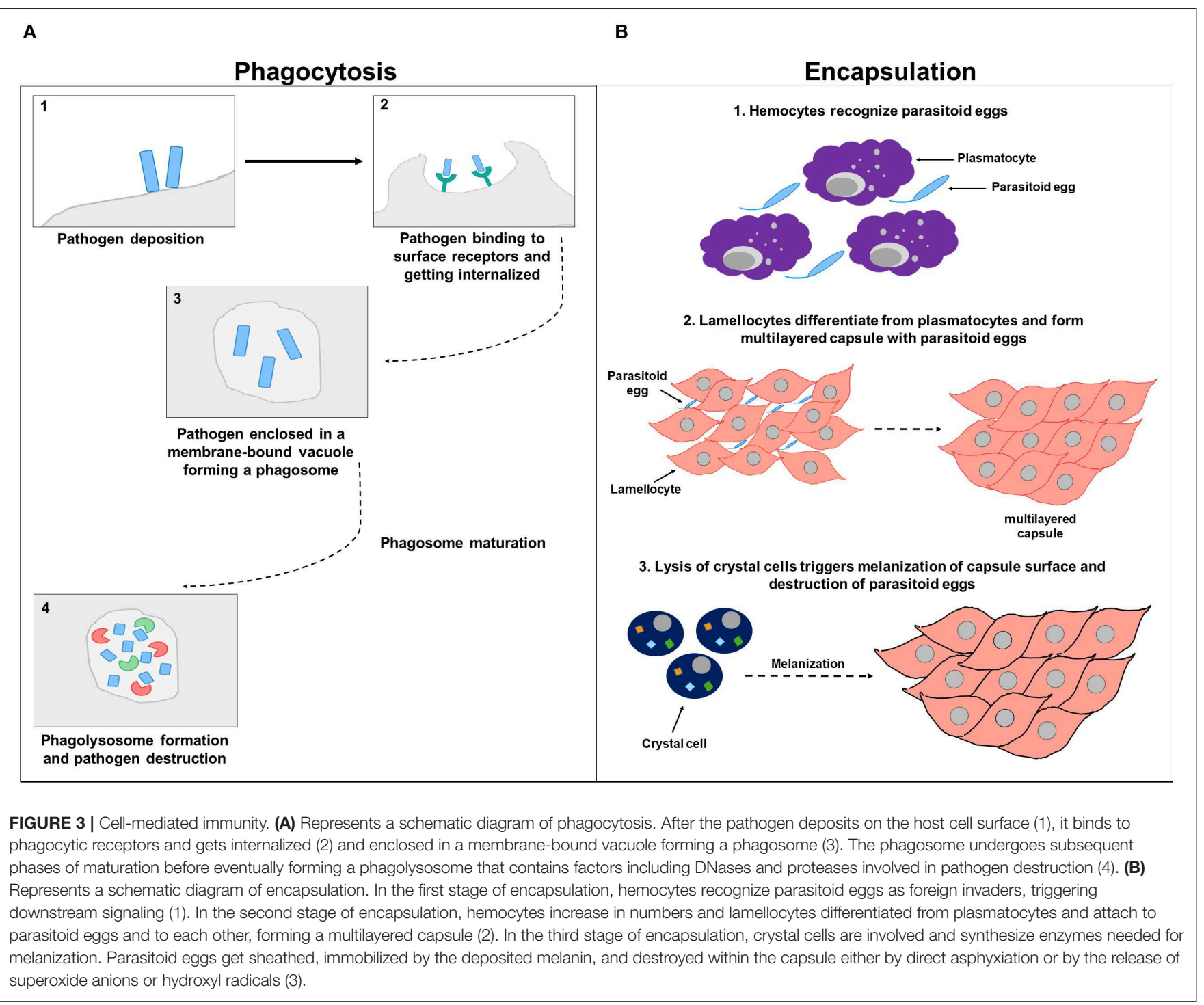

and Brey, 1995; Söderhäll and Cerenius, 1998; Cerenius et al., 2008). The melanotic reaction, which is generally induced by either a pathogenic infection or tissue injury, culminates in the proteolytic cleavage of inactive PPO to active phenol oxidase (PO), the chief enzyme in melanin biogenesis (Cerenius et al., 2008). To avoid the production of excessive intermediates that are toxic to the host, the activation of melanization is normally tightly regulated (De Gregorio et al., 2002b; Ligoxygakis et al., 2002b; Scherfer et al., 2008; Tang et al., 2008). In Drosophila, genetic studies identified melanization regulators including serine proteases and serpin proteins. MP1 and MP2/sp7/PAE1 clip proteases, for example, positively regulate melanization. Silencing either MP1 or MP2 inhibit PO activation upon pathogenic infection (De Gregorio et al., 2002b; Ligoxygakis et al., 2002b; Castillejo-Lopez and Hacker, 2005; Scherfer et al., 2008; Tang et al., 2008). Several studies also highlighted a role of PGRPs in inducing melanization. The proPO cascade in Drosophila larvae is induced by a forced expression of PGRP-LE, independent of infection. Consistent with this,
PGRP-LE is required for infection (E. coli)-induced melanization (Takehana et al., 2002, 2004). Likewise, PGRP-LC regulates melanization in Drosophila (Schmidt et al., 2007). Although melanization is considered an integral component in insect immunity, evidence of direct killing through quinone synthesis and melanin production has been reported in a few studies in insect species only. In Anopheles gamabiae, melanization was shown to retard Beauveria bassiana growth and dissemination (Yassine et al., 2012). In Manduca sexta, however, 60-94\% killing of a broad spectrum of gram-negative bacterial species including Klebsiella pneumoniae, Escherichia coli, Pseudomonas aeruginosa, and Salmonella typhimurium, and 52-99\% killing of gram-positive bacterial species including Staphylococcus aureus, Bacillus subtilis, Bacillus cereus, and Micrococcus luteus was reported in an active melanotic milieu (Zhao et al., 2007). Similarly, a recent study in $D$. melanogaster publicitized a novel role of melanization in anti-nematode immunity (Cooper et al., 2019). It is worth noting here that some host-pathogen interactions are "genotype by genotype" driven. Drosophila 
melanogaster's melanotic and complement-like immunity, for example, vary extensively against the parasitoid wasp Leptopilina boulardi. PO activity is predominantly affected by the host genotype, while TEP1 upregulation is controlled by the parasite genotype itself. Lamellocyte differentiation, on the other hand, depends on the specific combination of both the host and parasite genotypes (Leitão et al., 2019).

\section{RNA INTERFERENCE}

The RNA interference (RNAi) pathway, which suppresses gene expression through targeted RNA degradation, embodies an ancient mechanism of anti-viral immunity in plants, nematodes, and arthropods including Drosophila (Hamilton and Baulcombe, 1999; Li et al., 2002; Lu et al., 2005; Wilkins et al., 2005; Cherry and Silverman, 2006; Wang et al., 2006; Zambon et al., 2006; Saleh et al., 2009; Karlikow et al., 2014). This pathway emerges in two major phases including the "initiation" and the "execution" phase. Either endogenous (short hairpin RNAs manufactured by the genome, perversely expressed trans-genes, and transposons) or exogenous sources (naturally occurring or experimentally made dsRNA) can introduce dsRNA to initiate RNAi (Hannon, 2002; Zambon et al., 2006). dsRNA are recognized and cleaved by Dicer molecules to form small RNAs (Hammond et al., 2000; Blaszczyk et al., 2001; Zambon et al., 2006) that get integrated into the RNA-induced silencing complex (RISC), denoting the execution phase of the RNAi pathway (Blaszczyk et al., 2001; Nykanen et al., 2001; Zambon et al., 2006). Unlike mammals that have only one Dicer gene, and which is difficult to study, flies possess two genes, Dicer1 and Dicer2, that are required for processing miRNA precursors from pre-miRNA and siRNA precursors from long dsRNA, respectively (RoblesSikisaka et al., 2001). The single strand of either miRNA or siRNA integrated into the RISC complex acts as a platform for RISC to recognize complementary messenger RNA (mRNA) transcript. Upon recognition, Argonaute, one of the proteins in RISC, activates and cleaves the mRNA, inhibiting antiviral functions and suppressing viral expression (Karlikow et al., 2014) (Figure 4). Other existing, yet poorly identified, RNAi pathways include the PIWI-interacting RNA (piRNA) pathway that shields host cells from endogenous mobile genetic elements (Buchon et al., 2014). Several studies in Drosophila reported that loss-of-function mutations in essential RNAi pathway genes increase host vulnerability to viral infection (Zambon et al., 2006; Aliyari et al., 2008; Buchon et al., 2014). In mammals, other antiviral defense strategies, including protein sensors that recognize viral dsRNA motifs, have been identified. Among these sensors are the DEAD-box helicases RIG-I (Retinoic acid-inducible gene I) and MDA5 (Melanoma DifferentiationAssociated protein 5), together known as RIG-I-like receptors (RLRs). Upon recognizing viral nucleic acid during the primary viral infection stages, these sensors induce the expression of type 1 interferons (IFN $\alpha$ and IFN $\beta$ ) and other pro-inflammatory cytokines (Song and Rossi, 2017; van der Veen et al., 2018; Brisse and Ly, 2019). Interestingly, studies in Drosophila showed that Dicer-2 closely resembles the mammalian RLRs, not only by cleaving dsRNA into siRNA, but also by activating the transcription of antiviral effectors proteins (Deddouche et al.,
2008). Genetic screening in the fruit fly revealed additional antiviral roles of DEAD-box helicase. DDX17 (known as Rm62), for example, exhibits antiviral activity against arthropod-borne bunyaviruses (Deddouche et al., 2008). In addition to these nucleic acid-elicited responses, some viruses can be directly recognized by Toll-7, which promotes the activation of antiviral autophagy in an AKT pathway-dependent manner through phosphoinositide 3-kinase (PI3K) and target of rapamycin (Tor) (Buchon et al., 2014). Likewise, other studies in Drosophila also revealed a direct antiviral role of autophagy against the vesicular stomatitis virus, initiated by the pathogen surface glycoprotein VSVG (Shelly et al., 2009). Sabin et al. identified Ars2 (CG7843) as a key element of Drosophila antiviral immunity using an RNAi library and demonstrated that a loss of Ars2 function in either cells or flies promotes vulnerability to RNA viruses. In addition to its antiviral characteristic, Ars2 was shown to modulate Dcr-2 activity in vitro by physically interacting with it. It was also shown to play an essential role in siRNAand miRNA-mediated silencing. This crucial role of Ars2 in these small RNA pathways delivers novel insight into the biogenesis of small RNAs, a platform that could be extended to other systems (Sabin et al., 2009). Similarly, unrecognized host genes imperative for the influenza viral replication have been identified using genome-wide RNAi screens in Drosophila. Three of these identified genes have corresponding homologs in humans (ATP6V0D1, COX6A1, and NXF1). When tested in human HEK 293 cells, these genes were shown to be involved in the replication of $\mathrm{H} 5 \mathrm{~N} 1$ and $\mathrm{H} 1 \mathrm{~N} 1$ influenza $\mathrm{A}$ viruses, but not in vaccinia nor in vesicular stomatitis viral replication (Hao et al., 2008). The natural resistance-associated macrophage (NRAMP), a divalent metal ion transporter and a cell surface molecule expressed on Drosophila cells and required for binding and entry of sindbis virus to host cells, was also identified using RNAi technology. dNRAMP mutant flies were shown to be protected from viral infection (Rose et al., 2011). Interestingly, Carpenter et al. identified many differentially expressed genes in sigma virus-infected flies, several of which are neither up-regulated by bacterial or fungal infection, nor controlled by Toll, IMD, or JAK/STAT pathways, implying the involvement of other distinct regulatory immune mechanisms in defense against sigma virus in infected flies (Carpenter et al., 2009).

Ongoing studies are now applying genome-wide association study (GWAS) to identify the genetic basis of natural variation in Drosophila's immunity against pathogens. A study by Chapman et al. identified single nucleotide polymorphisms associated with genes (Bomanin gene BomBc1, krishah, and S6k) that significantly affected the fly's immunity to Enterococcus faecalis infection. Surprisingly, none of these genes are classified as canonical immune genes (Chapman et al., 2020).

Currently, the direction in unraveling host defense factors and innate immune effector molecules in the Drosophila model organism is heading toward bracketing classical genetic approaches with GWAS and genome-wide RNAi screening of flies with either loss of function or over-expressed immune genes, in addition to the use of co-immunoprecipitation assays and mass spectrometry to identify immune protein complexes. Moreover, bioinformatics analysis is being extensively adapted 


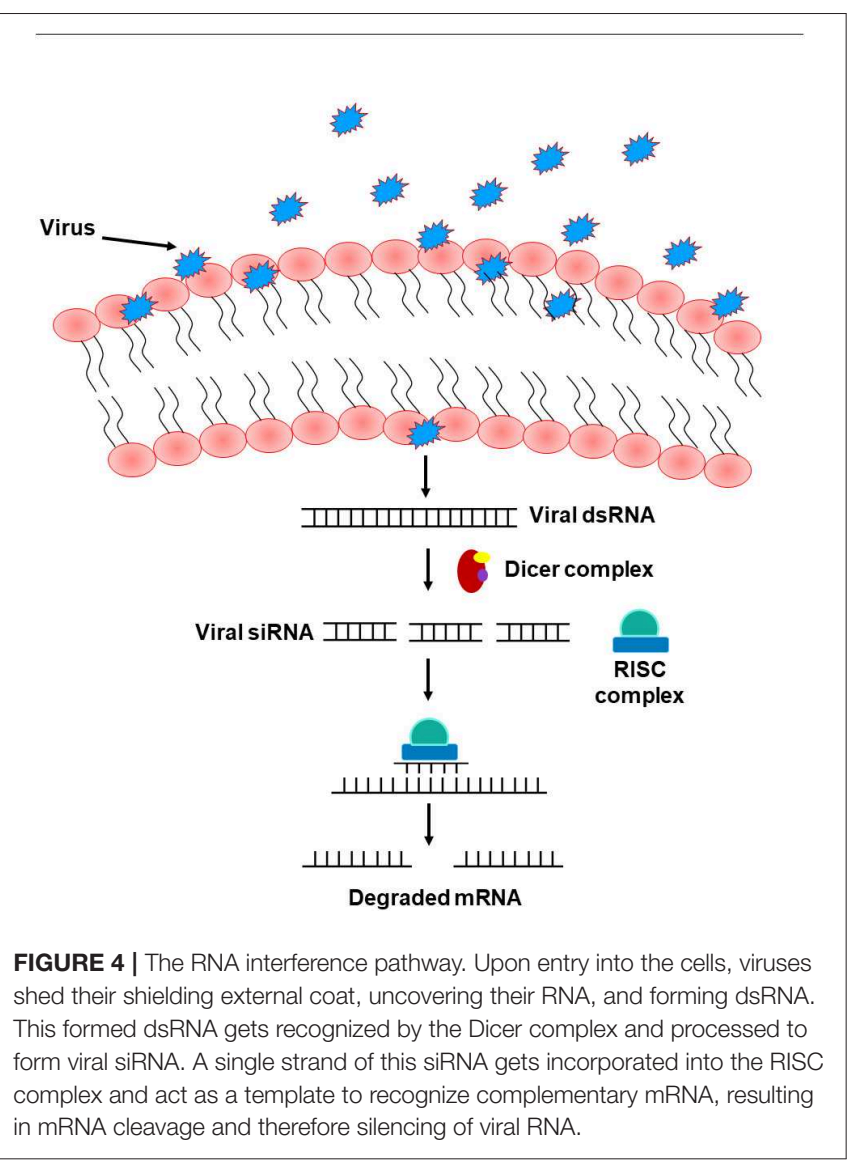

in deciphering candidate molecules and post-translational alterations that could impact the host's immune signaling pathways (Kanoh et al., 2019; Chapman et al., 2020). Nevertheless, and along with the ongoing high-throughput screens to discover conserved genes involved in host-pathogen interactions and immune signaling, the CRISPR/Cas9 technology has paved the way for additional wide-scale-based screens in Drosophila cultured cells, the results of which could be followed up in vivo in flies and/or mammals (Viswanatha et al., 2019). Some identified host factors required for defense against a broad spectrum of pathogens are summarized in Table $\mathbf{1 .}$

\section{PATHOGEN VIRULENCE FACTORS}

Diverse well-designed screening assay systems have been established to identify virulence factors contributing to pathogeninduced host killing using the Drosophila model organism. Screening for virulence-attenuated mutants identified a set of genes involved in the multi-host pathogenesis of $P$. aeruginosa PA14, for example. Follow-up studies further characterized these genes to validate the use of Drosophila as a model for high-throughput identification of novel virulence factors. Characterizing $h u d R$, an identified virulence gene encoding a MarR/SlyA family transcription factor, for instance, revealed that eminent expression of $h u d A$ (homologous to $U b i D$ ) is required and adequate to attenuate the virulence of hudR mutants in infected flies (Kim et al., 2008). Since quorum sensing is involved in the pathogenicity of $P$. aeruginosa, several studies focused on identifying quorum sensing-regulated virulence factors, as an appealing therapeutic approach to control $P$. aeruginosa infection (Bjarnsholt and Givskov, 2007). P. aeruginosa oxylipin lipids were also identified as pathogenic factors that promote bacterial virulence and biofilm formation in Drosophila (Martinez and Campos-Gomez, 2016). Further studies in P. aeruginosa also revealed that the phosphorylation state of the transcriptional response regulator $\mathrm{AlgR}$ inversely controls the production of pyoverdine and pyocyanin, two important $P$. aeruginosa virulence factors (Little et al., 2018). Other studies also focused on deciphering the effect of bacterial toxins on the host using fruit flies. P. aeruginosa exotoxin ExoS was shown to affect the activity of Rho GTPases, as the directed expression of the bacterial ExoS GAP domain (ExoSGAP) inhibits Rac1-, Cdc42-, and Rho-dependent signaling, suppressing Drosophila cellular immunity (Avet-Rochex et al., 2005). V. cholerae toxin, in turn, was shown to decrease intestinal stem cell division, alter epithelial regeneration, and induce cell-cell junctional damage (Guichard et al., 2013; Wang et al., 2013). Interestingly, the Vibrio polysaccharide (VPS)-dependent biofilm, which is highly activated upon entry into the arthropod intestine, is essential for Drosophila intestinal colonization (Purdy and Watnick, 2011). Surprisingly however, quorum sensing promotes a more auspicious interaction between the fly host and $V$. cholerae by reducing the nutritional burden of intestinal colonization in the host (Kamareddine et al., 2018b). Novel H. pylori effector proteins like the cytotoxin-associated gene A (CagA) have been studied in transgenic Drosophila flies. CagA mimics the eukaryotic adaptor protein Grb2-associated binder (Gab) and activates phosphatase SHP-2, a component of the receptor tyrosine kinase pathways. These findings in D. melanogaster could provide more insight into the role of translocated bacterial proteins that targets highly conserved eukaryotic cellular processes (Botham et al., 2008). The Anthrax toxin produced by Bacillus anthracis is comprised of protective antigen (PA), edema factor (EF), and lethal factor (LF) (Lacy and Collier, 2002). Similar to their function in mammals, LF cleaves MAPK kinases, and EF inhibits hedgehog pathway in flies (Guichard et al., 2006). This similarity in function strengthens the argument of choosing Drosophila as a multicellular host system to study in vivo function of virulence factors and diverse toxins. Drosophila has been also extensively used to study infectious properties of several bacterial species like Porphyromonas gingivalis (W83), a gram-negative obligate anaerobic bacteria strongly implicated in adult periodontitis (Griffen et al., 1998; Ezzo and Cutler, 2003; Igboin et al., 2011). P. gingivalis causes systemic infection in Drosophila and promotes potent killing in a dose-dependent manner. Interestingly, both heat-killed and live $P$. gingivalis are similarly pathogenic to the fly, suggesting a role of $P$. gingivalis cell surface components and Drosophila immunity in dictating pathology in this host-pathogen model (Igboin et al., 2011). Tabuchi et al. demonstrated an important role of $d l t A$, a gene responsible for D-alanylation of techoic acid in the cell wall of gram-positive $S$. aureus, in inhibiting the fly's Toll pathway. S. aureus-infected dltA mutant flies exhibited an increase in life span compared to flies expressing $d l t A$ normally 
TABLE 1 | Host defense factors.

\begin{tabular}{|c|c|c|c|c|}
\hline Pathogen & Host & Defense factor & Lessons from Drosophila & References \\
\hline $\begin{array}{l}\text { Bacillus thuringiensis } \\
\text { and } \\
\text { Erwinia carotovora carotovora }\end{array}$ & Adult flies & $\begin{array}{l}\text { Diuretic } \\
\text { Hormone } 31 \\
\text { (DH31) }\end{array}$ & $\begin{array}{l}\text { The DH31 enteroendocrine peptide stimulates gut } \\
\text { contractions, favoring the elimination of opportunistic } \\
\text { bacteria }\end{array}$ & Benguettat et al., 2018 \\
\hline $\begin{array}{l}\text { Erwinia carotovora } \\
\text { carotovora }\end{array}$ & Adult flies & $\begin{array}{l}\text { Drosophila } \\
\text { Peroxiredoxin V } \\
(\mathrm{dPrxV})\end{array}$ & $\begin{array}{l}\text { dPrXV mutant flies exhibit reduced survival after gut } \\
\text { infection } \\
\text { The JNK/FOXO signaling mediated expression of the } \\
\text { immune-related antioxidant enzyme dPrxV } \\
\text { plausibly protects the host gut epithelial cells from } \\
\text { oxidative damage during bacterial infection }\end{array}$ & Ahn et al., 2012 \\
\hline Klebsiella pneumoniae & Adult flies & Phg1 & Phg1 is implicated in resistance to Klebsiella infection & Benghezal et al., 2006 \\
\hline $\begin{array}{l}\text { Photorhabdus asymbiotica and } \\
\text { Photorhabdus luminescens }\end{array}$ & Adult flies & PGRP-LE & $\begin{array}{l}\text { PGRP-LE expression is upregulated following } \\
\text { Photorhabdus infection } \\
\text { Absence of functional PGRP-LE alters the transcriptional } \\
\text { pathway activity of JNK and IMD signaling upon infection } \\
\text { with Photorhabdus asymbiotica } \\
\text { PGRP-LE mutant flies are more sensitive to } \\
\text { Photorhabdus luminescens } \\
\text { Photorhabdus luminescens infection modifies the activity } \\
\text { of JAK/STAT signaling }\end{array}$ & Chevee et al., 2019 \\
\hline $\begin{array}{l}\text { Scedosporium apiospermum } \\
\text { and Scedosporium prolificans }\end{array}$ & Adult flies & Toll Pathway & $\begin{array}{l}\text { Wild-type flies are resistant to Scedosporium } \\
\text { apiospermum and Scedosporium } \\
\text { prolificans infections while Toll-deficiency results in acute } \\
\text { infection and high mortality rates }\end{array}$ & Lamaris et al., 2007 \\
\hline $\begin{array}{l}\text { Cryptococcus } \\
\text { neoformans }\end{array}$ & Adult flies & Toll Pathway & $\begin{array}{l}\text { The Toll pathway is necessary for clearing Cryptococcus } \\
\text { neoformans introduced directly into the fly hemolymph } \\
\text { and for the survival of systemically infected flies }\end{array}$ & Apidianakis et al., 2004 \\
\hline \multirow[t]{2}{*}{ Zygomycetes } & Adult flies & Toll Pathway & $\begin{array}{l}\text { Zygomycetes rapidly infect and kill wild-type flies and } \\
\text { Toll-deficient flies exhibit increased susceptibility to } \\
\text { Zygomycetes }\end{array}$ & Chamilos et al., 2008 \\
\hline & & Eater & $\begin{array}{l}\text { Phagocytosis impaired eater mutant flies exhibit } \\
\text { increased susceptibility to Zygomycetes infection }\end{array}$ & \\
\hline
\end{tabular}

(Tabuchi et al., 2010). Fungal virulence factors have been also reported in several Drosophila studies. Gliotoxin, for example, contributes to the virulence of Aspergillus fumigatus in fruit flies with functional phagocytes as well as in non-neutropenic mice, suggesting that gliotoxin principally targets neutrophils or other phagocytes (Spikes et al., 2008). Cas5 transcription in Candida albicans regulates cell wall integrity and is essential for fungal virulence in both murine and Toll mutant flies (Chamilos et al., 2009). Likewise, Candida glabrata mutant strains lacking the yapsin virulence factors or the high-osmolarity glycerol pathway exhibit a less virulent effect in infected flies (Quintin et al., 2013). Several antifungal drug efficacy studies against invasive aspergillosis (Lionakis et al., 2005) and malasseziosis (Merkel et al., 2018) have been conducted in the Drosophila model. Many studies have also demonstrated the ability of parasitic nematodes, like those belonging to the Heterorhabditis genus, to infect and kill fruit flies at larval and adult stages and to trigger an up-regulation in several genes belonging to the Toll, IMD, JAK/STAT, and TGF- $\beta$ signaling pathways (Castillo et al., 2013, 2015; Arefin et al., 2014). Heterorhabditis gerrardi, for example, harbors the pathogenic Photorhabdus asymbiotica bacteria, which gets ejected from the nematode gut into the host's hemolymph. In the hemolymph, Photorhabdus asymbiotica proliferates and releases toxins and virulence factors that kills the host and provides a favorable environment for the nematode (Waterfield et al., 2008; Eleftherianos et al., 2010). To analyze the impact of the Photorhabdus-Heterorhabditis mutualistic relation on the transcriptional profiles of the host, Castillo et al. (2015) performed next-generation RNAsequencing on flies infected with Photorhabdus alone, germfree Heterorhabditis lacking Photorhabdus, and Heterorhabditis carrying Photorhabdus. The bioinformatics analysis of that study revealed an impact of Photorhabdus on the transcription of fly genes associated with translational repression and stress responses, and an effect of Heterorhabditis on the expression profiles of genes involved in metabolism, lipid homeostasis, stress responses, DNA/protein synthesis, and functions of the nervous system.

In the last few years, a number of studies have examined virulence factors of human viral pathogens in Drosophila, as the fruit fly model facilitates the implementation of systematic, genome-wide RNAi analysis commonly used to identify genes that are involved in viral replication (Kuttenkeuler and Boutros, 2004). Since a broad spectrum of RNA viruses exploit internal ribosome entry sites (IRESs) for translation, genome-wide RNAi screen in Drosophila cells infected with Drosophila C virus were performed to reveal host factors required for IRES-dependent translation and viral replication. A study by Cherry et al. revealed 
TABLE 2 | Pathogen Virulence Factors.

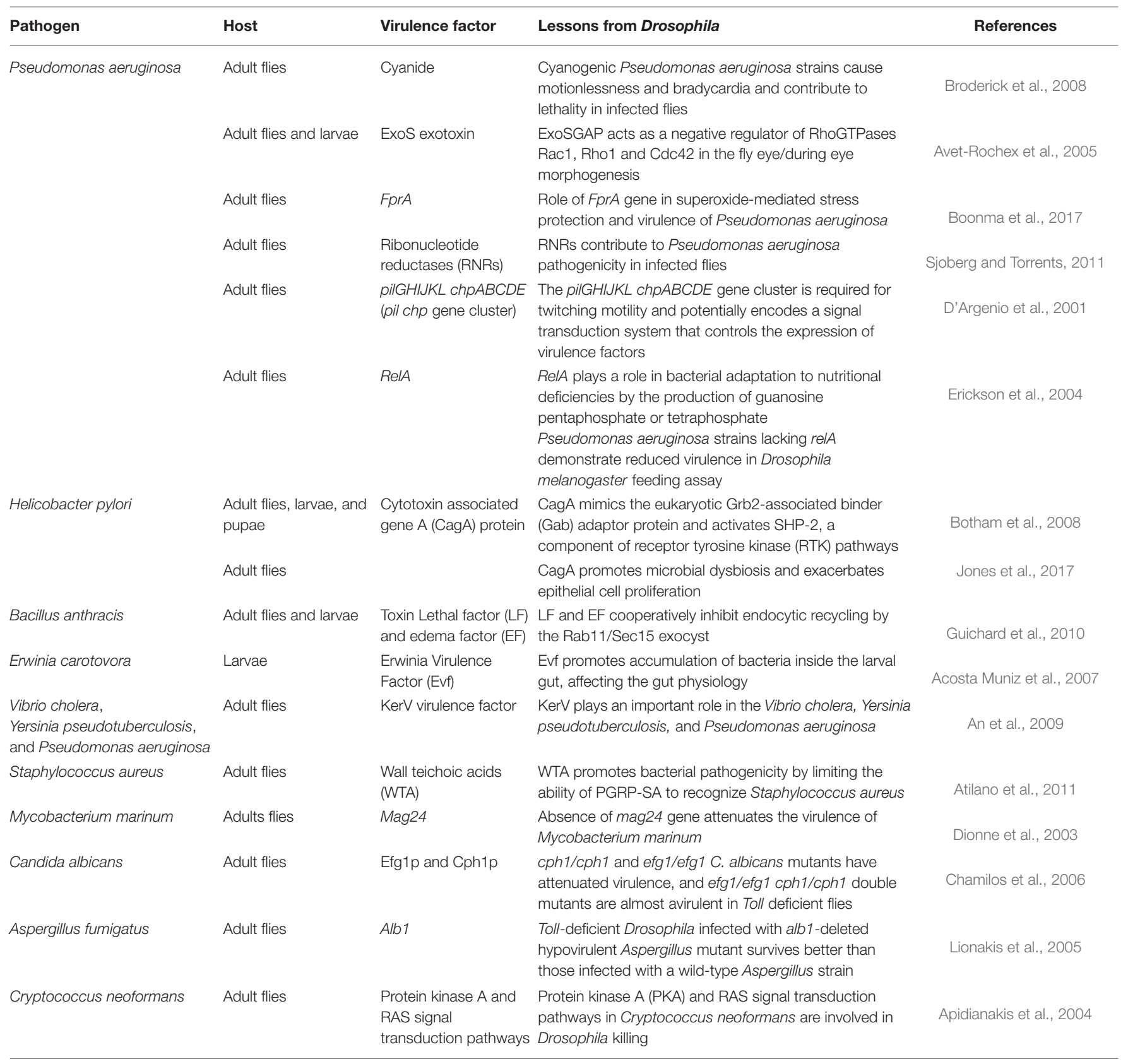

66 ribosomal proteins needed for Drosophila $\mathrm{C}$ virus, but not for non-IRES-containing RNA virus (Cherry et al., 2005). Some identified pathogen virulence factors are summarized in Table 2.

\section{CONCLUSION}

The use of animal models serves as a foundation to reveal conserved aspects of human disease. Unraveling detailed mechanisms of host-pathogen interactions using Drosophila provides further insight into the pathogenic arm of a microorganism and the defensive arm of a host. A better understanding of host-microbe interactions is desirable for the development of novel successful treatment regimens for pathogen-caused diseases.

\section{AUTHOR CONTRIBUTIONS}

All authors listed have made a substantial, direct and intellectual contribution to the work, and approved it for publication.

\section{ACKNOWLEDGMENTS}

We acknowledge Qatar University (QUST-1-CHS-2020-4) for funding this publication. 


\section{REFERENCES}

Acosta Muniz, C., Jaillard, D., Lemaitre, B., and Boccard, F. (2007). Erwinia carotovora Evf antagonizes the elimination of bacteria in the gut of Drosophila larvae. Cell Microbiol. 9, 106-119. doi: 10.1111/j.1462-5822.2006.00771.x

Adams, M. D., Celniker, S. E., Holt, R. A., Evans, C. A., Gocayne, J. D., Amanatides, P. G., et al. (2000). The genome sequence of Drosophila melanogaster. Science 287, 2185-2195. doi: 10.1126/science.287.5461.2185

Agaisse, H., Burrack, L. S., Philips, J. A., Rubin, E. J., Perrimon, N., and Higgins, D. E. (2005). Genome-wide RNAi screen for host factors required for intracellular bacterial infection. Science 309, 1248-1251. doi: 10.1126/science.11 16008

Agaisse, H., and Perrimon, N. (2004). The roles of JAK/STAT signaling in Drosophila immune responses. Immunol. Rev. 198, 72-82. doi: 10.1111/j.0105-2896.2004.0133.x

Ahn, H. M., Lee, K. S., Lee, D. S., and Yu, K. (2012). JNK/FOXO mediated PeroxiredoxinV expression regulates redox homeostasis during Drosophila melanogaster gut infection. Dev. Comp. Immunol. 38, 466-473. doi: 10.1016/j.dci.2012.07.002

Akira, S., Uematsu, S., and Takeuchi, O. (2006). Pathogen recognition and innate immunity. Cell 124, 783-801. doi: 10.1016/j.cell.2006.02.015

Aliyari, R., Wu, Q., Li, H. W., Wang, X. H., Li, F., Green, L. D., et al. (2008). Mechanism of induction and suppression of antiviral immunity directed by virus-derived small RNAs in Drosophila. Cell Host Microbe 4, 387-397. doi: 10.1016/j.chom.2008.09.001

An, D., Apidianakis, Y., Boechat, A. L., Baldini, R. L., Goumnerov, B. C., and Rahme, L. G. (2009). The pathogenic properties of a novel and conserved gene product, KerV, in proteobacteria. PLoS ONE 4:e7167. doi: 10.1371/journal.pone.0007167

Anjum, S. G., Xu, W., Nikkholgh, N., Basu, S., Nie, Y., Thomas, M., et al. (2013). Regulation of Toll signaling and inflammation by $\beta$-arrestin and the SUMO protease Ulp1. Genetics 195, 1307-1317. doi: 10.1534/genetics.113.157859

Apidianakis, Y., Rahme, L. G., Heitman, J., Ausubel, F. M., Calderwood, S. B., and Mylonakis, E. (2004). Challenge of Drosophila melanogaster with Cryptococcus neoformans and role of the innate immune response. Eukaryot. Cell 3, 413-419. doi: 10.1128/EC.3.2.413-419.2004

Arefin, B., Kucerova, L., Dobes, P., Markus, R., Strnad, H., Wang, Z., et al. (2014). Genome-wide transcriptional analysis of Drosophila larvae infected by entomopathogenic nematodes shows involvement of complement, recognition and extracellular matrix proteins. J. Innate Immun. 6, 192-204. doi: $10.1159 / 000353734$

Ashida, M., and Brey, P. T. (1995). Role of the integument in insect defense: prophenol oxidase cascade in the cuticular matrix. Proc. Natl. Acad. Sci. U.S.A. 92, 10698-10702. doi: 10.1073/pnas.92.23.10698

Atilano, M. L., Yates, J., Glittenberg, M., Filipe, S. R., and Ligoxygakis, P. (2011). Wall teichoic acids of Staphylococcus aureus limit recognition by the drosophila peptidoglycan recognition protein-SA to promote pathogenicity. PLoS Pathog. 7:e1002421. doi: 10.1371/journal.ppat.1002421

Avadhanula, V., Weasner, B. P., Hardy, G. G., Kumar, J. P., and Hardy, R. W. (2009). A novel system for the launch of alphavirus RNA synthesis reveals a role for the Imd pathway in arthropod antiviral response. PLoS Pathog. 5:e1000582. doi: 10.1371/journal.ppat.1000582

Avet-Rochex, A., Bergeret, E., Attree, I., Meister, M., and Fauvarque, M. O. (2005). Suppression of Drosophila cellular immunity by directed expression of the ExoS toxin GAP domain of Pseudomonas aeruginosa. Cell Microbiol. 7, 799-810. doi: 10.1111/j.1462-5822.2005.00512.x

Aymeric, J. L., Givaudan, A., and Duvic, B. (2010). Imd pathway is involved in the interaction of Drosophila melanogaster with the entomopathogenic bacteria, Xenorhabdus nematophila and Photorhabdus luminescens. Mol. Immunol. 47, 2342-2348. doi: 10.1016/j.molimm.2010.05.012

Benghezal, M., Fauvarque, M. O., Tournebize, R., Froquet, R., Marchetti, A., Bergeret, E., et al. (2006). Specific host genes required for the killing of Klebsiella bacteria by phagocytes. Cell Microbiol. 8, 139-148. doi: 10.1111/j.1462-5822.2005.00607.x

Benguettat, O., Jneid, R., Soltys, J., Loudhaief, R., Brun-Barale, A., Osman, D., et al. (2018). The DH31/CGRP enteroendocrine peptide triggers intestinal contractions favoring the elimination of opportunistic bacteria. PLoS Pathog. 14:e1007279. doi: 10.1371/journal.ppat.1007279
Bier, E., and Guichard, A. (2012). Deconstructing host-pathogen interactions in Drosophila. Dis. Models Mech. 5, 48-61. doi: 10.1242/dmm.000406

Bjarnsholt, T., and Givskov, M. (2007). The role of quorum sensing in the pathogenicity of the cunning aggressor Pseudomonas aeruginosa. Anal. Bioanal. Chem. 387, 409-414. doi: 10.1007/s00216-006-0774-x

Blandin, S., Shiao, S.-H., Moita, L. F., Janse, C. J., Waters, A. P., Kafatos, F. C., et al. (2004). Complement-like protein TEP1 is a determinant of vectorial capacity in the malaria vector Anopheles gambiae. Cell 116, 661-670. doi: 10.1016/S0092-8674(04)00173-4

Blaszczyk, J., Tropea, J. E., Bubunenko, M., Routzahn, K. M., Waugh, D. S., Court, D. L., et al. (2001). Crystallographic and modeling studies of RNase III suggest a mechanism for double-stranded RNA cleavage. Structure 9, 1225-1236. doi: 10.1016/S0969-2126(01)00685-2

Boonma, S., Romsang, A., Duang-Nkern, J., Atichartpongkul, S., Trinachartvanit, W., Vattanaviboon, P., et al. (2017). The FinR-regulated essential gene fprA, encoding ferredoxin NADP+ reductase: roles in superoxide-mediated stress protection and virulence of Pseudomonas aeruginosa. PLoS ONE 12:e0172071. doi: 10.1371/journal.pone.0172071

Botham, C. M., Wandler, A. M., and Guillemin, K. (2008). A transgenic Drosophila model demonstrates that the Helicobacter pylori CagA protein functions as a eukaryotic Gab adaptor. PLoS Pathog. 4:e1000064. doi: 10.1371/journal.ppat.1000064

Brand, A. H., and Perrimon, N. (1993). Targeted gene expression as a means of altering cell fates and generating dominant phenotypes. Development 118, 401-415.

Bretscher, A. J., Honti, V., Binggeli, O., Burri, O., Poidevin, M., Kurucz, E., et al. (2015). The Nimrod transmembrane receptor Eater is required for hemocyte attachment to the sessile compartment in Drosophila melanogaster. Biol. Open 4, 355-363. doi: 10.1242/bio.201410595

Brisse, M., and Ly, H. (2019). Comparative structure and function analysis of the RIG-I-like receptors: RIG-I and MDA5. Front. Immunol. 10:1586. doi: 10.3389/fimmu.2019.01586

Broderick, K. E., Chan, A., Balasubramanian, M., Feala, J., Reed, S. L., Panda, M., et al. (2008). Cyanide produced by human isolates of Pseudomonas aeruginosa contributes to lethality in Drosophila melanogaster. J. Infect. Dis. 197, 457-464. doi: $10.1086 / 525282$

Buchon, N., Broderick, N. A., Chakrabarti, S., and Lemaitre, B. (2009b). Invasive and indigenous microbiota impact intestinal stem cell activity through multiple pathways in Drosophila. Genes Dev. 23, 2333-2344. doi: 10.1101/gad.1827009

Buchon, N., Broderick, N. A., Poidevin, M., Pradervand, S., and Lemaitre, B. (2009c). Drosophila intestinal response to bacterial infection: activation of host defense and stem cell proliferation. Cell Host Microbe 5, 200-211. doi: 10.1016/j.chom.2009.01.003

Buchon, N., Poidevin, M., Kwon, H. M., Guillou, A., Sottas, V., Lee, B. L., et al. (2009a). A single modular serine protease integrates signals from patternrecognition receptors upstream of the Drosophila Toll pathway. Proc. Natl. Acad. Sci. U.S.A. 106, 12442-12447. doi: 10.1073/pnas.0901924106

Buchon, N., Silverman, N., and Cherry, S. (2014). Immunity in Drosophila melanogaster-from microbial recognition to whole-organism physiology. Nat. Rev. Immunol. 14, 796-810. doi: 10.1038/nri3763

Carpenter, J., Hutter, S., Baines, J. F., Roller, J., Saminadin-Peter, S. S., Parsch, J., et al. (2009). The transcriptional response of Drosophila melanogaster to infection with the sigma virus (Rhabdoviridae). PLoS ONE 4:e6838. doi: 10.1371 /journal.pone. 0006838

Castillejo-Lopez, C., and Hacker, U. (2005). The serine protease Sp7 is expressed in blood cells and regulates the melanization reaction in Drosophila. Biochem. Biophys. Res. Commun. 338, 1075-1082. doi: 10.1016/j.bbrc.2005.10.042

Castillo, J. C., Creasy, T., Kumari, P., Shetty, A., Shokal, U., Tallon, L. J., et al. (2015). Drosophila anti-nematode and antibacterial immune regulators revealed by RNA-Seq. BMC Genomics 16:519. doi: 10.1186/s12864-015$1690-2$

Castillo, J. C., Shokal, U., and Eleftherianos, I. (2013). Immune gene transcription in Drosophila adult flies infected by entomopathogenic nematodes and their mutualistic bacteria. J. Insect. Physiol. 59, 179-185. doi: 10.1016/j.jinsphys.2012.08.003

Cerenius, L., Lee, B. L., and Söderhäll, K. (2008). The proPO-system: pros and cons for its role in invertebrate immunity. Trends Immunol. 29, 263-271. doi: $10.1016 /$ j.it.2008.02.009 
Chamilos, G., Bignell, E. M., Schrettl, M., Lewis, R. E., Leventakos, K., May, G. S., et al. (2010). Exploring the concordance of Aspergillus fumigatus pathogenicity in mice and Toll-deficient flies. Med. Mycol. 48, 506-510. doi: $10.3109 / 13693780903225813$

Chamilos, G., Lewis, R. E., Hu, J., Xiao, L., Zal, T., Gilliet, M., et al. (2008). Drosophila melanogaster as a model host to dissect the immunopathogenesis of zygomycosis. Proc. Natl. Acad. Sci. U.S.A. 105, 9367-9372. doi: 10.1073/pnas.0709578105

Chamilos, G., Lionakis, M. S., Lewis, R. E., Lopez-Ribot, J. L., Saville, S. P., Albert, N. D., et al. (2006). Drosophila melanogaster as a facile model for largescale studies of virulence mechanisms and antifungal drug efficacy in Candida species. J. Infect. Dis. 193, 1014-1022. doi: 10.1086/500950

Chamilos, G., Nobile, C. J., Bruno, V. M., Lewis, R. E., Mitchell, A. P., and Kontoyiannis, D. P. (2009). Candida albicans Cas5, a regulator of cell wall integrity, is required for virulence in murine and toll mutant fly models. J. Infect. Dis. 200, 152-157. doi: 10.1086/599363

Chapman, J. R., Dowell, M. A., Chan, R., and Unckless, R. L. (2020). The genetic basis of natural variation in Drosophila melanogaster immune defense against Enterococcus faecalis. Genes 11:234. doi: 10.3390/genes11020234

Cherry, S. (2008). Genomic RNAi screening in Drosophila S2 cells: what have we learned about host-pathogen interactions? Curr. Opin. Microbiol. 11, 262-270. doi: 10.1016/j.mib.2008.05.007

Cherry, S., Doukas, T., Armknecht, S., Whelan, S., Wang, H., Sarnow, P., et al. (2005). Genome-wide RNAi screen reveals a specific sensitivity of IREScontaining RNA viruses to host translation inhibition. Genes Dev. 19, 445-452. doi: $10.1101 /$ gad.1267905

Cherry, S., and Silverman, N. (2006). Host-pathogen interactions in drosophila: new tricks from an old friend. Nat. Immunol. 7, 911-917. doi: 10.1038/ni1388

Chevee, V., Sachar, U., Yadav, S., Heryanto, C., and Eleftherianos, I. (2019). The peptidoglycan recognition protein PGRP-LE regulates the Drosophila immune response against the pathogen Photorhabdus. Microb. Pathog. 136:103664. doi: 10.1016/j.micpath.2019.103664

Christensen, B. M., Li, J., Chen, C.-C., and Nappi, A. J. (2005). Melanization immune responses in mosquito vectors. Trends Parasitol. 21, 192-199. doi: $10.1016 / j . p t .2005 .02 .007$

Cooper, D., Wuebbolt, C., Heryanto, C., and Eleftherianos, I. (2019). The prophenoloxidase system in Drosophila participates in the anti-nematode immune response. Mol. Immunol. 109, 88-98. doi: 10.1016/j.molimm.2019.03.008

Costa, A., Jan, E., Sarnow, P., and Schneider, D. (2009). The Imd pathway is involved in antiviral immune responses in Drosophila. PLoS ONE 4:e7436. doi: 10.1371/journal.pone.0007436

Cronin, S. J., Nehme, N. T., Limmer, S., Liegeois, S., Pospisilik, J. A., Schramek, D., et al. (2009). Genome-wide RNAi screen identifies genes involved in intestinal pathogenic bacterial infection. Science 325, 340-343. doi: 10.1126/science.1173164

Cuttell, L., Vaughan, A., Silva, E., Escaron, C. J., Lavine, M., Van Goethem, E., et al. (2008). Undertaker, a Drosophila Junctophilin, links Drapermediated phagocytosis and calcium homeostasis. Cell 135, 524-534. doi: 10.1016/j.cell.2008.08.033

D’Argenio, D. A., Gallagher, L. A., Berg, C. A., and Manoil, C. (2001). Drosophila as a model host for Pseudomonas aeruginosa infection. J. Bacteriol. 183, 1466-1471. doi: 10.1128/JB.183.4.1466-1471.2001

De Gregorio, E., Han, S.-J., Lee, W.-J., Baek, M.-J., Osaki, T., Kawabata, S.-I., et al. (2002b). An immune-responsive Serpin regulates the melanization cascade in Drosophila. Dev. Cell 3, 581-592. doi: 10.1016/S1534-5807(02)00267-8

De Gregorio, E., Spellman, P. T., Tzou, P., Rubin, G. M., and Lemaitre, B. (2002a). The Toll and Imd pathways are the major regulators of the immune response in Drosophila. EMBO J. 21, 2568-2579. doi: 10.1093/emboj/21.11.2568

Deddouche, S., Matt, N., Budd, A., Mueller, S., Kemp, C., Galiana-Arnoux, D., et al. (2008). The DExD/H-box helicase Dicer-2 mediates the induction of antiviral activity in drosophila. Nat. Immunol. 9, 1425-1432. doi: 10.1038/ni.1664

Dionne, M. S., Ghori, N., and Schneider, D. S. (2003). Drosophila melanogaster is a genetically tractable model host for Mycobacterium marinum. Infect. Immun. 71, 3540-3550. doi: 10.1128/IAI.71.6.3540-3550.2003

Dionne, M. S., and Schneider, D. S. (2008). Models of infectious diseases in the fruit fly Drosophila melanogaster. Dis. Models Mech. 1, 43-49. doi: $10.1242 / \mathrm{dmm} .000307$
Dostert, C., Jouanguy, E., Irving, P., Troxler, L., Galiana-Arnoux, D., Hetru, C., et al. (2005). The Jak-STAT signaling pathway is required but not sufficient for the antiviral response of drosophila. Nat. Immunol. 6, 946-953. doi: $10.1038 /$ ni1237

Eleftherianos, I., ffrench-Constant, R. H., Clarke, D. J., Dowling, A. J., and Reynolds, S. E. (2010). Dissecting the immune response to the entomopathogen Photorhabdus. Trends Microbiol. 18, 552-560. doi: 10.1016/j.tim.2010.09.006

Engel, E., Viargues, P., Mortier, M., Taillebourg, E., Couté, Y., Thevenon, D., et al. (2014). Identifying USPs regulating immune signals in Drosophila: USP2 deubiquitinates Imd and promotes its degradation by interacting with the proteasome. Cell Commun. Signal. 12:41. doi: 10.1186/s12964-014-0041-2

Erickson, D. L., Lines, J. L., Pesci, E. C., Venturi, V., and Storey, D. G. (2004). Pseudomonas aeruginosa relA contributes to virulence in Drosophila melanogaster. Infect. Immun. 72, 5638-5645. doi: 10.1128/IAI.72.10.5638-5645.2004

Ezzo, P. J., and Cutler, C. W. (2003). Microorganisms as risk indicators for periodontal disease. Periodontology (2000) 32, 24-35. doi: 10.1046/j.0906-6713.2003.03203.x

Fellowes, M. D., and Godfray, H. C. (2000). The evolutionary ecology of resistance to parasitoids by Drosophila. Heredity (Edinb). 84, 1-8. doi: $10.1046 /$ j.1365-2540.2000.00685.x

Franc, N. C., Heitzler, P., Ezekowitz, R. A., and White, K. (1999). Requirement for croquemort in phagocytosis of apoptotic cells in Drosophila. Science 284, 1991-1994. doi: 10.1126/science.284.5422.1991

Fullaondo, A., Garcia-Sanchez, S., Sanz-Parra, A., Recio, E., Lee, S. Y., and Gubb, D. (2011). Spn1 regulates the GNBP3-dependent Toll signaling pathway in Drosophila melanogaster. Mol. Cell Biol. 31, 2960-2972. doi: 10.1128/MCB.01397-10

Galiana-Arnoux, D., Dostert, C., Schneemann, A., Hoffmann, J. A., and Imler, J.L. (2006). Essential function in vivo for Dicer-2 in host defense against RNA viruses in drosophila. Nat. Immunol. 7, 590-597. doi: 10.1038/ni1335

Garver, L. S., Wu, J., and $\mathrm{Wu}, \mathrm{L}$. P. (2006). The peptidoglycan recognition protein PGRP-SC1a is essential for Toll signaling and phagocytosis of Staphylococcus aureus in Drosophila. Proc. Natl. Acad. Sci. U.S.A. 103, 660-665. doi: 10.1073/pnas. 0506182103

Gottar, M., Gobert, V., Matskevich, A. A., Reichhart, J.-M., Wang, C., Butt, T. M., et al. (2006). Dual detection of fungal infections in Drosophila via recognition of glucans and sensing of virulence factors. Cell 127, 1425-1437. doi: 10.1016/j.cell.2006.10.046

Govind, S. (2008). Innate immunity in Drosophila: pathogens and pathways. Insect Sci. 15, 29-43. doi: 10.1111/j.1744-7917.2008.00185.x

Griffen, A. L., Becker, M. R., Lyons, S. R., Moeschberger, M. L., and Leys, E. J. (1998). Prevalence of Porphyromonas gingivalis and periodontal health status. J. Clin. Microbiol. 36, 3239-3242. doi: 10.1128/JCM.36.11.3239-3242.1998

Guichard, A., Cruz-Moreno, B., Aguilar, B., van Sorge, N. M., Kuang, J., Kurkciyan, A. A., et al. (2013). Cholera toxin disrupts barrier function by inhibiting exocyst-mediated trafficking of host proteins to intestinal cell junctions. Cell Host Microbe 14, 294-305. doi: 10.1016/j.chom.2013.09.003

Guichard, A., McGillivray, S. M., Cruz-Moreno, B., van Sorge, N. M., Nizet, V., and Bier, E. (2010). Anthrax toxins cooperatively inhibit endocytic recycling by the Rab11/Sec15 exocyst. Nature 467, 854-858. doi: 10.1038/nature09446

Guichard, A., Park, J. M., Cruz-Moreno, B., Karin, M., and Bier, E. (2006). Anthrax lethal factor and edema factor act on conserved targets in Drosophila. Proc. Natl. Acad. Sci. U.S.A. 103, 3244-3249. doi: 10.1073/pnas.0510748103

Haasnoot, J., and Berkhout, B. (2006). "RNA interference: its use as antiviral therapy," in RNA Towards Medicine, eds V. Erdmann, J. Barciszewski, and J. Brosius (Berlin: Springer), 117-150. doi: 10.1007/3-540-27262-3_7

Hamilton, A. J., and Baulcombe, D. C. (1999). A species of small antisense RNA in posttranscriptional gene silencing in plants. Science 286, 950-952. doi: $10.1126 /$ science.286.5441.950

Hammond, S. M., Bernstein, E., Beach, D., and Hannon, G. J. (2000). An RNAdirected nuclease mediates post-transcriptional gene silencing in Drosophila cells. Nature 404, 293-296. doi: 10.1038/35005107

Hannon, G. J. (2002). RNA interference. Nature 418, 244-251. doi: $10.1038 / 418244$ a

Hao, L., Sakurai, A., Watanabe, T., Sorensen, E., Nidom, C. A., Newton, M. A., et al. (2008). Drosophila RNAi screen identifies host genes important for influenza virus replication. Nature 454, 890-893. doi: 10.1038/nature07151 
Hedengren-Olcott, M., Olcott, M. C., Mooney, D. T., Ekengren, S., Geller, B. L., and Taylor, B. J. (2004). Differential activation of the NF-kappaB-like factors Relish and Dif in Drosophila melanogaster by fungi and Gram-positive bacteria. J. Biol. Chem. 279, 21121-21127. doi: 10.1074/jbc.M313856200

Herbein, G., and O'Brien, W. A. (2000). Tumor necrosis factor (TNF)-alpha and TNF receptors in viral pathogenesis. Proc. Soc. Exp. Biol. Med. 223, 241-257. doi: $10.1111 / j .1525-1373.2000 .22335 . x$

Hori, A., Kurata, S., and Kuraishi, T. (2018). Unexpected role of the IMD pathway in Drosophila gut defense against Staphylococcus aureus. Biochem. Biophys. Res. Commun. 495, 395-400. doi: 10.1016/j.bbrc.2017.11.004

Igboin, C. O., Moeschberger, M. L., Griffen, A. L., and Leys, E. J. (2011). Porphyromonas gingivalis virulence in a Drosophila melanogaster model. Infect. Immun. 79, 439-448. doi: 10.1128/IAI.00784-10

Jiang, H., Patel, P. H., Kohlmaier, A., Grenley, M. O., McEwen, D. G., and Edgar, B. A. (2009). Cytokine/Jak/Stat signaling mediates regeneration and homeostasis in the Drosophila midgut. Cell 137, 1343-1355. doi: 10.1016/j.cell.2009.05.014

Jones, T. A., Hernandez, D. Z., Wong, Z. C., Wandler, A. M., and Guillemin, K. (2017). The bacterial virulence factor CagA induces microbial dysbiosis that contributes to excessive epithelial cell proliferation in the Drosophila gut. PLoS Pathog. 13:e1006631. doi: 10.1371/journal.ppat.1006631

Kamareddine, L., Robins, W. P., Berkey, C. D., Mekalanos, J. J., and Watnick, P. I. (2018a). The Drosophila immune deficiency pathway modulates Enteroendocrine function and host metabolism. Cell Metab. 28, 449-462.e5. doi: 10.1016/j.cmet.2018.05.026

Kamareddine, L., Wong, A. C. N., Vanhove, A. S., Hang, S., Purdy, A. E., Kierek-Pearson, K., et al. (2018b). Activation of Vibrio cholerae quorum sensing promotes survival of an arthropod host. Nat. Microbiol. 3, 243-252. doi: 10.1038/s41564-017-0065-7

Kanoh, H., Kato, H., Suda, Y., Hori, A., Kurata, S., and Kuraishi, T. (2019). Dual comprehensive approach to decipher the Drosophila Toll pathway, ex vivo RNAi screenings and immunoprecipitation-mass spectrometry. Biochem. Biophys. Res. Commun. 508, 332-337. doi: 10.1016/j.bbrc.2018.11.007

Karlikow, M., Goic, B., and Saleh, M. C. (2014). RNAi and antiviral defense in Drosophila: setting up a systemic immune response. Dev. Comp. Immunol. 42, 85-92. doi: 10.1016/j.dci.2013.05.004

Kawai, T., and Akira, S. (2006). Innate immune recognition of viral infection. Nat. Immunol. 7, 131-137. doi: 10.1038/ni1303

Kemp, C., Mueller, S., Goto, A., Barbier, V., Paro, S., Bonnay, F., et al. (2013). Broad RNA interference-mediated antiviral immunity and virusspecific inducible responses in Drosophila. J. Immunol. 190, 650-658. doi: 10.4049/jimmunol.1102486

Kiger, A. A., Baum, B., Jones, S., Jones, M. R., Coulson, A., Echeverri, C., et al. (2003). A functional genomic analysis of cell morphology using RNA interference. J. Biol. 2:27. doi: 10.1186/1475-4924-2-27

Kim, S. H., Park, S. Y., Heo, Y. J., and Cho, Y. H. (2008). Drosophila melanogasterbased screening for multihost virulence factors of Pseudomonas aeruginosa PA14 and identification of a virulence-attenuating factor, HudA. Infect. Immun. 76, 4152-4162. doi: 10.1128/IAI.01637-07

Kocks, C., Cho, J. H., Nehme, N., Ulvila, J., Pearson, A. M., Meister, M., et al. (2005). Eater, a transmembrane protein mediating phagocytosis of bacterial pathogens in Drosophila. Cell 123, 335-346. doi: 10.1016/j.cell.2005.08.034

Koo, I. C., Ohol, Y. M., Wu, P., Morisaki, J. H., Cox, J. S., and Brown, E. J. (2008). Role for lysosomal enzyme beta-hexosaminidase in the control of mycobacteria infection. Proc. Natl. Acad. Sci. U.S.A. 105, 710-715. doi: 10.1073/pnas.0708110105

Kounatidis, I., and Ligoxygakis, P. (2012). Drosophila as a model system to unravel the layers of innate immunity to infection. Open Biol. 2:120075. doi: 10.1098/rsob.120075

Kuttenkeuler, D., and Boutros, M. (2004). Genome-wide RNAi as a route to gene function in Drosophila. Brief. Funct. Genomic Proteomic 3, 168-176. doi: $10.1093 / \mathrm{bfgp} / 3.2 .168$

Kuttenkeuler, D., Pelte, N., Ragab, A., Gesellchen, V., Schneider, L., Blass, C., et al. (2010). A large-scale RNAi screen identifies Deaf1 as a regulator of innate immune responses in Drosophila. J. Innate Immun. 2, 181-194. doi: $10.1159 / 000248649$

Lacy, D. B., and Collier, R. J. (2002). Structure and function of anthrax toxin. Curr. Top. Microbiol. Immunol. 271, 61-85. doi: 10.1007/978-3-662-05767-4_4
Lamaris, G. A., Chamilos, G., Lewis, R. E., and Kontoyiannis, D. P. (2007). Virulence studies of Scedosporium and Fusarium species in Drosophila melanogaster. J. Infect. Dis. 196, 1860-1864. doi: 10.1086/523765

Leitão, A. B., Bian, X., Day, J. P., Pitton, S., Demir, E., and Jiggins, F. M. (2019). Independent effects on cellular and humoral immune responses underlie genotype-by-genotype interactions between Drosophila and parasitoids. PLoS Pathog. 15:e1008084. doi: 10.1371/journal.ppat.1008084

Lemaitre, B., and Hoffmann, J. (2007). The host defense of Drosophila melanogaster. Annu. Rev. Immunol. 25, 697-743. doi: 10.1146/annurev.immunol.25.022106.141615

Leulier, F., Rodriguez, A., Khush, R. S., Abrams, J. M., and Lemaitre, B. (2000). The Drosophila caspase Dredd is required to resist gram-negative bacterial infection. EMBO Rep. 1, 353-358. doi: 10.1093/embo-reports/kvd073

Levashina, E. A., Moita, L. F., Blandin, S., Vriend, G., Lagueux, M., and Kafatos, F. C. (2001). Conserved role of a complement-like protein in phagocytosis revealed by dsRNA knockout in cultured cells of the mosquito, Anopheles gambiae. Cell 104, 709-718. doi: 10.1016/S0092-8674(01)00267-7

$\mathrm{Li}, \mathrm{H}$., Li, W. X., and Ding, S. W. (2002). Induction and suppression of RNA silencing by an animal virus. Science 296, 1319-1321. doi: 10.1126/science.1070948

Liao, J.-F., Wu, C.-P., Tang, C.-K., Tsai, C.-W., Rouhová, L., and Wu, Y.-L. (2019). Identification of regulatory host genes involved in sigma virus replication using RNAi knockdown in Drosophila. Insects 10:339. doi: 10.3390/insects10100339

Ligoxygakis, P., Bulet, P., and Reichhart, J.-M. (2002a). Critical evaluation of the role of the Toll-like receptor 18-Wheeler in the host defense of Drosophila. EMBO Rep. 3, 666-673. doi: 10.1093/embo-reports/kvf130

Ligoxygakis, P., Pelte, N., Ji, C., Leclerc, V., Duvic, B., Belvin, M., et al. (2002b). A serpin mutant links Toll activation to melanization in the host defence of Drosophila. EMBO J. 21, 6330-6337. doi: 10.1093/emboj/cdf661

Lionakis, M. S., Lewis, R. E., May, G. S., Wiederhold, N. P., Albert, N. D., Halder, G., et al. (2005). Toll-deficient Drosophila flies as a fast, high-throughput model for the study of antifungal drug efficacy against invasive aspergillosis and Aspergillus virulence. J. Infect. Dis. 191, 1188-1195. doi: 10.1086/ 428587

Little, A. S., Okkotsu, Y., Reinhart, A. A., Damron, F. H., Barbier, M., Barrett, B., et al. (2018). Pseudomonas aeruginosa AlgR phosphorylation status differentially regulates pyocyanin and pyoverdine production. MBio 9:e0231817. doi: $10.1128 / \mathrm{mBio} .02318-17$

Lu, R., Maduro, M., Li, F., Li, H. W., Broitman-Maduro, G., Li, W. X., et al. (2005). Animal virus replication and RNAi-mediated antiviral silencing in Caenorhabditis elegans. Nature 436, 1040-1043. doi: 10.1038/nature 03870

Martinez, E., and Campos-Gomez, J. (2016). Oxylipins produced by Pseudomonas aeruginosa promote biofilm formation and virulence. Nat. Commun. 7:13823. doi: 10.1038/ncomms 13823

Medvedev, A. E., Murphy, M., Zhou, H., and Li, X. (2015). E3 ubiquitin ligases Pellinos as regulators of pattern recognition receptor signaling and immune responses. Immunol. Rev. 266, 109-122. doi: 10.1111/imr.12298

Medzhitov, R. (2001). Toll-like receptors and innate immunity. Nat. Rev. Immunol. 1, 135-145. doi: 10.1038/35100529

Meister, G., and Tuschl, T. (2004). Mechanisms of gene silencing by doublestranded RNA. Nature 431, 343-349. doi: 10.1038/nature02873

Meister, M., and Richards, G. (1996). Ecdysone and insect immunity: the maturation of the inducibility of the diptericin gene in Drosophila larvae. Insect Biochem. Mol. Biol. 26, 155-160. doi: 10.1016/0965-1748(95)00076-3

Merkel, S., Heidrich, D., Danilevicz, C. K., Scroferneker, M. L., and Zanette, R. A. (2018). Drosophila melanogaster as a model for the study of Malassezia pachydermatis infections. Vet. Microbiol. 224, 31-33. doi: 10.1016/j.vetmic.2018.08.021

Miyano-Kurosaki, N., and Takaku, H. (2006). "Gene silencing of virus replication by RNA interference," in RNA Towards Medicine, eds V. Erdmann, J. Barciszewski, and J. Brosius (Poznan: Springer), 151-171. doi: 10.1007/3-540-27262-3_8

Myllymaki, H., and Ramet, M. (2014). JAK/STAT pathway in Drosophila immunity. Scand. J. Immunol. 79, 377-385. doi: 10.1111/sji.12170

Nappi, A. J., Carton, Y., and Frey, F. (1991). Parasite-induced enhancement of hemolymph tyrosinase activity in a selected immune reactive strain 
of Drosophila melanogaster. Archiv. Insect Biochem. Physiol. 18, 159-168. doi: 10.1002/arch.940180304

Nappi, A. J., and Vass, E. (1998). Hydrogen peroxide production in immune-reactive Drosophila melanogaster. J. Parasitol. 84, 1150-1157. doi: $10.2307 / 3284664$

Nappi, A. J., Vass, E., Frey, F., and Carton, Y. (1995). Superoxide anion generation in Drosophila during melanotic encapsulation of parasites. Eur. J. Cell Biol. $68,450-456$.

Nappi, A. J., Vass, E., Frey, F., and Carton, Y. (2000). Nitric oxide involvement in Drosophila immunity. Nitric Oxide 4, 423-430. doi: 10.1006/niox.2000.0294

Nusslein-Volhard, C., and Wieschaus, E. (1980). Mutations affecting segment number and polarity in Drosophila. Nature 287, 795-801. doi: $10.1038 / 287795 \mathrm{a} 0$

Nykanen, A., Haley, B., and Zamore, P. D. (2001). ATP requirements and small interfering RNA structure in the RNA interference pathway. Cell 107, 309-321. doi: 10.1016/S0092-8674(01)00547-5

Oh, C. T., Moon, C., Jeong, M. S., Kwon, S. H., and Jang, J. (2013). Drosophila melanogaster model for Mycobacterium abscessus infection. Microbes Infect. 15, 788-795. doi: 10.1016/j.micinf.2013.06.011

Paradkar, P. N., Trinidad, L., Voysey, R., Duchemin, J. B., and Walker, P. J. (2012). Secreted Vago restricts West Nile virus infection in Culex mosquito cells by activating the Jak-STAT pathway. Proc. Natl. Acad. Sci. U.S.A. 109, 18915-18920. doi: 10.1073/pnas.1205231109

Pearson, A., Lux, A., and Krieger, M. (1995). Expression cloning of dSR-CI, a class C macrophage-specific scavenger receptor from Drosophila melanogaster. Proc. Natl. Acad. Sci. U.S.A. 92, 4056-4060. doi: 10.1073/pnas.92.9.4056

Pfeiffer, B. D., Ngo, T.-T. B., Hibbard, K. L., Murphy, C., Jenett, A., Truman, J. W., et al. (2010). Refinement of tools for targeted gene expression in Drosophila. Genetics 186, 735-755. doi: 10.1534/genetics.110.119917

Pham, L. N., Dionne, M. S., Shirasu-Hiza, M., and Schneider, D. S. (2007). A specific primed immune response in Drosophila is dependent on phagocytes. PLoS Pathog. 3:e26. doi: 10.1371/journal.ppat.0030026

Purdy, A. E., and Watnick, P. I. (2011). Spatially selective colonization of the arthropod intestine through activation of Vibrio cholerae biofilm formation. Proc. Natl. Acad. Sci. U.S.A. 108, 19737-19742. doi: 10.1073/pnas.1111530108

Quintin, J., Asmar, J., Matskevich, A. A., Lafarge, M. C., and Ferrandon, D. (2013). The Drosophila Toll pathway controls but does not clear Candida glabrata infections. J. Immunol. 190, 2818-2827. doi: 10.4049/jimmunol.12 01861

Ramet, M., Manfruelli, P., Pearson, A., Mathey-Prevot, B., and Ezekowitz, R. A. (2002). Functional genomic analysis of phagocytosis and identification of a Drosophila receptor for E. coli. Nature 416, 644-648. doi: 10.1038/ nature 735

Rizki, R. M., and Rizki, T. M. (1990). Parasitoid virus-like particles destroy Drosophila cellular immunity. Proc. Natl. Acad. Sci. U.S.A. 87, 8388-8392. doi: $10.1073 /$ pnas.87.21.8388

Robles-Sikisaka, R., Garcia, D. K., Klimpel, K. R., and Dhar, A. K. (2001). Nucleotide sequence of $3^{\prime}$-end of the genome of Taura syndrome virus of shrimp suggests that it is related to insect picornaviruses. Arch. Virol. 146, 941-952. doi: 10.1007/s007050170126

Rose, P. P., Hanna, S. L., Spiridigliozzi, A., Wannissorn, N., Beiting, D. P., Ross, S. R., et al. (2011). Natural resistance-associated macrophage protein is a cellular receptor for sindbis virus in both insect and mammalian hosts. Cell Host Microbe 10, 97-104. doi: 10.1016/j.chom.2011.06.009

Rosetto, M., Engstrom, Y., Baldari, C. T., Telford, J. L., and Hultmark, D. (1995). Signals from the IL-1 receptor homolog, Toll, can activate an immune response in a Drosophila hemocyte cell line. Biochem. Biophys. Res. Commun. 209, 111-116. doi: 10.1006/bbrc.1995.1477

Sabin, L. R., Zhou, R., Gruber, J. J., Lukinova, N., Bambina, S., Berman, A., et al. (2009). Ars2 regulates both miRNA- and siRNA-dependent silencing and suppresses RNA virus infection in Drosophila. Cell 138, 340-351. doi: 10.1016/j.cell.2009.04.045

Saleh, M. C., Tassetto, M., van Rij, R. P., Goic, B., Gausson, V., Berry, B., et al. (2009). Antiviral immunity in Drosophila requires systemic RNA interference spread. Nature 458, 346-350. doi: 10.1038/nature07712

Salt, G. (1970). The Cellular Defence Reactions of Insects. Vol. 16. Cambridge: Cambridge University Press.
Scherfer, C., Tang, H., Kambris, Z., Lhocine, N., Hashimoto, C., and Lemaitre, B. (2008). Drosophila Serpin-28D regulates hemolymph phenoloxidase activity and adult pigmentation. Dev. Biol. 323, 189-196. doi: 10.1016/j.ydbio.2008.08.030

Schmidt, R. L. (2014). A roadmap to understanding toll pathway changes: an educational primer for use with "regulation of toll signaling and inflammation by $\beta$-arrestin and the SUMO protease Ulp1". Genetics 196, 923-929. doi: 10.1534/genetics.114.162289

Schmidt, R. L., Trejo, T. R., Plummer, T. B., Platt, J. L., and Tang, A. H. (2007). Infection-induced proteolysis of PGRP-LC controls the IMD activation and melanization cascades in Drosophila. FASEB J. 22, 918-929. doi: 10.1096/fj.06-7907com

Shelly, S., Lukinova, N., Bambina, S., Berman, A., and Cherry, S. (2009). Autophagy is an essential component of Drosophila immunity against vesicular stomatitis virus. Immunity 30, 588-598. doi: 10.1016/j.immuni.2009.02.009

Shiratsuchi, A., Mori, T., Sakurai, K., Nagaosa, K., Sekimizu, K., Lee, B. L., et al. (2012). Independent recognition of Staphylococcus aureus by two receptors for phagocytosis in Drosophila. J. Biol. Chem. 287, 21663-21672. doi: 10.1074/jbc.M111.333807

Sjoberg, B. M., and Torrents, E. (2011). Shift in ribonucleotide reductase gene expression in Pseudomonas aeruginosa during infection. Infect. Immun. 79, 2663-2669. doi: 10.1128/IAI.01212-10

Söderhäll, K., and Cerenius, L. (1998). Role of the prophenoloxidaseactivating system in invertebrate immunity. Curr. Opin. Immunol. 10, 23-28. doi: 10.1016/s0952-7915(98)80026-5

Song, M.-S., and Rossi, J. J. (2017). Molecular mechanisms of Dicer: endonuclease and enzymatic activity. Biochem. J. 474, 1603-1618. doi: 10.1042/BCJ20160759

Spikes, S., Xu, R., Nguyen, C. K., Chamilos, G., Kontoyiannis, D. P., Jacobson, R. H., et al. (2008). Gliotoxin production in Aspergillus fumigatus contributes to host-specific differences in virulence. J. Infect. Dis. 197, 479-486. doi: $10.1086 / 525044$

Strand, M. R., and Pech, L. L. (1995). Immunological basis for compatibility in parasitoid-host relationships. Annu. Rev. Entomol. 40, 31-56. doi: 10.1146/annurev.en.40.010195.000335

Stroschein-Stevenson, S. L., Foley, E., O'Farrell, P. H., and Johnson, A. D. (2006). Identification of Drosophila gene products required for phagocytosis of Candida albicans. PLoS Biol. 4:e4. doi: 10.1371/journal.pbio.00 40004

Tabuchi, Y., Shiratsuchi, A., Kurokawa, K., Gong, J. H., Sekimizu, K., Lee, B. L., et al. (2010). Inhibitory role for D-alanylation of wall teichoic acid in activation of insect Toll pathway by peptidoglycan of Staphylococcus aureus. J. Immunol. 185, 2424-2431. doi: 10.4049/jimmunol.1000625

Takehana, A., Katsuyama, T., Yano, T., Oshima, Y., Takada, H., Aigaki, T., et al. (2002). Overexpression of a pattern-recognition receptor, peptidoglycanrecognition protein-LE, activates imd/relish-mediated antibacterial defense and the prophenoloxidase cascade in Drosophila larvae. Proc. Natl. Acad. Sci. U.S.A. 99, 13705-13710. doi: 10.1073/pnas.212301199

Takehana, A., Yano, T., Mita, S., Kotani, A., Oshima, Y., and Kurata, S. (2004). Peptidoglycan recognition protein (PGRP)-LE and PGRP-LC act synergistically in Drosophila immunity. EMBO J. 23, 4690-4700. doi: 10.1038/sj.emboj.7600466

Tang, H., Kambris, Z., Lemaitre, B., and Hashimoto, C. (2008). A serpin that regulates immune melanization in the respiratory system of Drosophila. Dev. Cell 15, 617-626. doi: 10.1016/j.devcel.2008.08.017

Thevenon, D., Engel, E., Avet-Rochex, A., Gottar, M., Bergeret, E., Tricoire, H., et al. (2009). The Drosophila ubiquitin-specific protease dUSP36/Scny targets IMD to prevent constitutive immune signaling. Cell Host Microbe 6, 309-320. doi: 10.1016/j.chom.2009.09.007

Tsichritzis, T., Gaentzsch, P., Kosmidis, S., Brown, A., Skoulakis, E., Ligoxygakis, P., et al. (2007). A Drosophila ortholog of the human cylindromatosis tumor suppressor gene regulates triglyceride content and antibacterial defense. Development 134, 2605-2614. doi: 10.1242/dev.02859

Ueda, R. (2001). RNAi: a new technology in the post-genomic sequencing era. $J$. Neurogenet. 15, 193-204. doi: 10.3109/01677060109167376

van der Veen, A. G., Maillard, P. V., Schmidt, J. M., Lee, S. A., DeddoucheGrass, S., Borg, A., et al. (2018). The RIG-I-like receptor LGP2 inhibits Dicer-dependent processing of long double-stranded RNA and blocks RNA 
interference in mammalian cells. EMBO J. 37:e97479. doi: 10.15252/embj.2017 97479

Viswanatha, R., Brathwaite, R., Hu, Y., Li, Z., Rodiger, J., Merckaert, P., et al. (2019). Pooled CRISPR Screens in Drosophila cells. Curr. Protoc. Mol. Biol. 129:e111. doi: $10.1002 / \mathrm{cpmb} .111$

Vodovar, N., Vinals, M., Liehl, P., Basset, A., Degrouard, J., Spellman, P., et al. (2005). Drosophila host defense after oral infection by an entomopathogenic Pseudomonas species. Proc. Natl. Acad. Sci. U.S.A. 102, 11414-11419. doi: 10.1073/pnas.0502240102

Wang, X. H., Aliyari, R., Li, W. X., Li, H. W., Kim, K., Carthew, R., et al. (2006). RNA interference directs innate immunity against viruses in adult Drosophila. Science 312, 452-454. doi: 10.1126/science.1125694

Wang, Z., Hang, S., Purdy, A. E., and Watnick, P. I. (2013). Mutations in the IMD pathway and mustard counter Vibrio cholerae suppression of intestinal stem cell division in Drosophila. MBio 4:e00337-13. doi: 10.1128/mBio.00337-13

Waterfield, N., Sanchez-Contreras, M., Eleftherianos, I., Dowling, A., Yang, G., Wilkinson, P., et al. (2008). Rapid virulence annotation (RVA): identification of virulence factors using a bacterial genome library and multiple invertebrate hosts. Proc. Natl. Acad. Sci. U.S.A. 105, 15967-15972. doi: $10.1073 /$ pnas.0711114105

Wilkins, C., Dishongh, R., Moore, S. C., Whitt, M. A., Chow, M., and Machaca, K. (2005). RNA interference is an antiviral defence mechanism in Caenorhabditis elegans. Nature 436, 1044-1047. doi: 10.1038/nature03957

Yagi, R., Mayer, F., and Basler, K. (2010). Refined LexA transactivators and their use in combination with the Drosophila Gal4 system. Proc. Natl. Acad. Sci. U.S.A. 107, 16166-16171. doi: 10.1073/pnas.1005957107

Yang, H., Kronhamn, J., Ekstrom, J. O., Korkut, G. G., and Hultmark, D. (2015). JAK/STAT signaling in Drosophila muscles controls the cellular immune response against parasitoid infection. EMBO Rep. 16, 1664-1672. doi: 10.15252/embr.201540277

Yassine, H., Kamareddine, L., and Osta, M. A. (2012). The mosquito melanization response is implicated in defense against the entomopathogenic fungus Beauveria bassiana. PLoS Pathog. 8:e1003029. doi: 10.1371/journal.ppat.1003029

Zambon, R. A., Nandakumar, M., Vakharia, V. N., and Wu, L. P. (2005). The Toll pathway is important for an antiviral response in Drosophila. Proc. Natl. Acad. Sci. U.S.A. 102, 7257-7262. doi: 10.1073/pnas.0409181102

Zambon, R. A., Vakharia, V. N., and Wu, L. P. (2006). RNAi is an antiviral immune response against a dsRNA virus in Drosophila melanogaster. Cell Microbiol. 8, 880-889. doi: 10.1111/j.1462-5822.2006.00688.x

Zhao, P., Li, J., Wang, Y., and Jiang, H. (2007). Broad-spectrum antimicrobial activity of the reactive compounds generated in vitro by Manduca sexta phenoloxidase. Insect Biochem. Mol. Biol. 37, 952-959. doi: 10.1016/j.ibmb.2007.05.001

Conflict of Interest: The authors declare that the research was conducted in the absence of any commercial or financial relationships that could be construed as a potential conflict of interest.

Copyright (c) 2020 Younes, Al-Sulaiti, Nasser, Najjar and Kamareddine. This is an open-access article distributed under the terms of the Creative Commons Attribution License (CC BY). The use, distribution or reproduction in other forums is permitted, provided the original author(s) and the copyright owner(s) are credited and that the original publication in this journal is cited, in accordance with accepted academic practice. No use, distribution or reproduction is permitted which does not comply with these terms. 


\section{OPEN ACCESS}

Edited by:

Gheyath Khaled Nasrallah, Qatar University, Qatar

Reviewed by:

Valeria Rachela Villella, Italian Institute of Technology (IIT), Italy

Don Thushara Galbadage, Biola University, United States

*Correspondence:

Laurent Kremer laurent.kremer@irim.cnrs.fr

Specialty section:

This article was submitted to

Bacteria and Host

a section of the journal

Frontiers in Cellular and Infection

Microbiology

Received: 27 April 2020

Accepted: 10 June 2020

Published: 17 July 2020

Citation:

Johansen MD and Kremer L (2020)

CFTR Depletion Confers

Hypersusceptibility to Mycobacterium fortuitum in a Zebrafish Model.

Front. Cell. Infect. Microbiol. 10:357.

doi: 10.3389/fcimb.2020.00357

\section{CFTR Depletion Confers Hypersusceptibility to Mycobacterium fortuitum in a Zebrafish Model}

\author{
Matt D. Johansen ${ }^{1}$ and Laurent Kremer ${ }^{1,2 *}$ \\ ${ }^{1}$ Institut de Recherche en Infectiologie de Montpellier, Centre National de la Recherche Scientifique UMR 9004, Université de \\ Montpellier, Montpellier, France, ${ }^{2}$ INSERM, Institut de Recherche en Infectiologie de Montpellier, Montpellier, France
}

The Mycobacterium fortuitum complex comprises several closely related species, causing pulmonary and extra-pulmonary infections. However, there is very limited knowledge about the disease pathogenesis involved in $M$. fortuitum infections, particularly due to the lack of suitable animal models. Using the zebrafish model, we show that embryos are susceptible to $M$. fortuitum infection in a dose-dependent manner. Furthermore, zebrafish embryos form granulomas from as early as 2 days post-infection, recapitulating critical aspects of mycobacterial pathogenesis observed in other pathogenic species. The formation of extracellular cords in infected embryos highlights a previously unknown pathogenic feature of $M$. fortuitum. The formation of large corded structures occurs also during in vitro growth, suggesting that this is not a host-adapted stress mechanism deployed during infection. Moreover, transient macrophage depletion led to rapid embryo death with increased extracellular cords, indicating that macrophages are essential determinants of $M$. fortuitum infection control. Importantly, morpholino depletion of the cystic fibrosis transmembrane conductance regulator (cftr) significantly increased embryo death, bacterial burden, bacterial cords and abscesses. There was a noticeable decrease in the number of cftr-deficient infected embryos with granulomas as compared to infected controls, suggesting that loss of CFTR leads to impaired host immune responses and confers hypersusceptiblity to M. fortuitum infection. Overall, these findings highlight the application of the zebrafish embryo to study $M$. fortuitum and emphasizes previously unexplored aspects of disease pathogenesis of this significant mycobacterial species.

\footnotetext{
Keywords: Mycobacterium fortuitum, granuloma, infection, cording, cystic fibrosis, CFTR, pathogenesis, zebrafish
}

\section{INTRODUCTION}

The incidence of non-tuberculous mycobacterial (NTM) infections is increasing globally, surpassing the infection rate of tuberculosis in many developed countries (Johansen et al., 2020). NTM are increasingly acknowledged pathogens capable of infecting a vast array of tissues and inducing a wide spectrum of clinical symptoms in humans, with pulmonary infections being the most common clinical manifestations (Ratnatunga et al., 2020). NTM infections are particularly 
prevalent in immunocompromised individuals and those with underlying genetic and structural defects. They have emerged in recent years as prevalent pathogens in cystic fibrosis (CF) patients, with several global CF centers reporting the isolation of NTM in the respiratory tract of CF patients (Roux et al., 2009; Skolnik et al., 2016). NTM prevalence in CF patients varies between 5 and 20\%, with the Mycobacterium avium complex and the Mycobacterium abscessus complex being the most significant species (Richards and Olivier, 2019). However, less frequently isolated species in CF patients include other NTM, such as Mycobacterium gordonae, Mycobacterium kansasii or M. fortuitum (Sermet-Gaudelus et al., 2003; Cândido et al., 2014; Martiniano et al., 2016; Richards and Olivier, 2019).

The $M$. fortuitum complex includes $M$. fortuitum, Mycobacterium peregrinum, Mycobacterium porcinum, Mycobacterium septicum, Mycobacterium conceptionense, Mycobacterium boenickei, Mycobacterium houstonense, Mycobacterium neworleansense, Mycobacterium brisbanense, Mycobacterium farcinogenes, Mycobacterium senegalense, and Mycobacterium setense (Brown-Elliott and Wallace, 2002; Brown-Elliott and Philley, 2017; Tortoli et al., 2017). $M$. fortuitum is a rapid-growing and frequently identified NTM species, causing localized skin and soft tissue infections (Wallace et al., 1983). Several clinical cases have also reported catheter infections, post-surgical infections, peritonitis, eye infections, and pulmonary infections (Brown-Elliott et al., 2012; Brown-Elliott and Philley, 2017). Unfortunately, our understanding surrounding the pathogenicity of $M$. fortuitum has been hampered by the lack of genetic tools and by the restricted panel of cellular and animal models available. Among the few animal models reported, a murine infection model was characterized by a sustained persistent infection of M. fortuitum in the kidneys (Parti et al., 2005, 2008). However, while the bacilli proliferated freely inside murine macrophages they did not invade a murine kidney cell line (Parti et al., 2005). A murine neutropenic model, in which neutropenia is induced by intraperitoneal doses of cyclophosphamide, has also been exploited to demonstrate the in vivo efficacy of drugs against M. fortuitum and M. abscessus (Das et al., 2019). In addition, a recent study highlighted the suitability of the Galleria mellonella moth larvae as a cheap, efficient and rapid in vivo model for the screening of antibiotic combinations and novel treatments against NTM, including $M$. fortuitum (Entwistle and Coote, 2018; García-Coca et al., 2019).

Infection of mice with $M$. fortuitum are also accompanied by visible symptoms, such as spinning disease (Saito and Tasaka, 1969; Parti et al., 2005), often associated with neurological disorders. Interestingly, spinning disease due to invasive abscess formation within the central nervous system has been described both in mice as well as in zebrafish embryos infected with M. abscessus (Saito and Tasaka, 1969; Bernut et al., 2014a), suggesting that both $M$. abscessus and M. fortuitum display common pathogenic traits in these animals. Interestingly, M. fortuitum, formerly Mycobacterium ranae, was originally recovered from frogs in 1905 and considered a pathogen for animals and humans since its first isolation from a human abscess in 1938. Together with Mycobacterium marinum, it is commonly associated with fish tuberculosis, a systemic and chronic disease characterized by the presence of granulomatous reactions. Supporting the view that $M$. fortuitum infects amphibians and fish, inoculation in adult goldfish led to the development of a characteristic chronic granulomatous response similar to that associated with natural mycobacterial infection (Talaat et al., 1999). Overall, these observations suggest that zebrafish may represent a valuable model to study pathogenesis of this understudied mycobacterial species.

Zebrafish have recently gained favor as a useful and amenable model to study host-bacterial interactions (Davis et al., 2002; van der Sar et al., 2004; Clay et al., 2007; Prajsnar et al., 2008, 2013; Vergunst et al., 2010; Alibaud et al., 2011; Cambier et al., 2014; Gomes and Mostowy, 2020). Due to genetic tractability and optical transparency, zebrafish embryos represent an exquisite model to study important aspects of infectious diseases. Whilst the adult zebrafish possess a complex immune system similar to that of humans, comprising both the innate and adaptive arms of immunity, the early embryonic stages solely harbor innate immunity (Davis et al., 2002). Infection of embryos, thus allows a more in-depth focus on the role of innate immunity during the very early stages of infection (Davis et al., 2002; Torraca and Mostowy, 2018). Zebrafish infection with the fish pathogen $M$. marinum has led to remarkable insights into the understanding of human tuberculosis (Davis et al., 2002; Berg and Ramakrishnan, 2012), the role of macrophages in pathogen dissemination (Davis and Ramakrishnan, 2009), infection-induced antibiotic tolerance (Adams et al., 2011), the contribution of ESX secretion system in granuloma formation (Volkman et al., 2004) and induced granuloma-associated angiogenesis which promotes mycobacterial growth and facilitates the spread of infection to distant tissue sites (Oehlers et al., 2015). However, a noticeably important breakthrough came from the $M$. abscessus model of infection in zebrafish, which unraveled the importance of cording as mechanism of immune evasion (Bernut et al., 2014a) and the role of TNF signaling in controlling infection (Bernut et al., 2016a). Because many conserved virulence mechanisms and host susceptibility determinants identified during zebrafish infection have been validated in humans (Tobin et al., 2010; Bernut et al., 2019), we reasoned that zebrafish may also represent a useful experimental model to decipher the virulence and immunopathology of $M$. fortuitum infections.

Herein, we have exploited the zebrafish embryo as an amenable model for the study of systemic $M$. fortuitum infections. We describe the aggressive and lethal infections caused by $M$. fortuitum, which develops in the absence of either functional innate immunity or CFTR.

\section{MATERIALS AND METHODS}

\section{Mycobacterial Strains and Culture Conditions}

Mycobacterium abscessus CIP104536 ${ }^{\mathrm{T}}$, M. fortuitum subsp. fortuitum (ATCC 6841) and Mycobacterium smegmatis $\mathrm{mc}^{2} 155$ were routinely grown and maintained at $37^{\circ} \mathrm{C}$ in Middlebrook 
7H9 broth (BD Difco) supplemented with $10 \%$ oleic acid, albumin, dextrose, catalase (OADC; BD Difco) and $0.025 \%$ Tyloxapol (Sigma-Aldrich) $\left(7 \mathrm{H} 9^{\mathrm{OADC} / \mathrm{T}}\right)$ or on Middlebrook $7 \mathrm{H} 10$ supplemented with $10 \%$ OADC enrichment ( $\left.7 \mathrm{H} 10^{\mathrm{OADC}}\right)$ and in the presence of antibiotics if required. To observe in vitro liquid growth phenotypes, bacteria were grown in Cationadjusted Mueller-Hinton Broth (CaMHB; Sigma-Aldrich).

\section{Creation of Fluorescent $\boldsymbol{M}$. fortuitum}

Fluorescent M. fortuitum was generated using the pVV16eGFP replicative vector (Vilchèze et al., 2014). Electrocompetent $M$. fortuitum were generated as previously described (Viljoen et al., 2018). M. fortuitum was placed on ice for $2 \mathrm{~h}$ prior to pelleting by centrifugation $(3,000 \times \mathrm{g}$ at $4^{\circ} \mathrm{C}$ for $15 \mathrm{~min}$ ). Following centrifugation, bacteria were resuspended in decreasing volumes of wash buffer $(10 \%$ glycerol $(\mathrm{v} / \mathrm{v})$ and $0.025 \%$ Tyloxapol in distilled water) and pelleted by centrifugation for a total of 4 washes. For electroporation transformation, $1 \mu \mathrm{g}$ of plasmid DNA was added to $200 \mu \mathrm{L}$ of electrocompetent bacteria and transferred to a chilled $0.2 \mathrm{~cm}$ electrode gap GenePulser electroporation cuvette (Bio-rad) and transformed using a GenePulser Cxell electroporator (Bio-rad) $(2.5 \mathrm{kV}, 1,000 \Omega$ and $25 \mu \mathrm{F})$. Bacteria were recovered in $800 \mu \mathrm{L}$ of $7 \mathrm{H} 9^{\mathrm{OADC} / \mathrm{T}}$ and placed at $37^{\circ} \mathrm{C}$ overnight. For selection of green fluorescent colonies, M. fortuitum was plated on $7 \mathrm{H} 10^{\mathrm{OADC}}$ supplemented $50 \mu \mathrm{g} / \mathrm{mL}$ kanamycin (Euromedex). Positive colonies were selected based on fluorescence and maintained in $7 \mathrm{H} 9^{\mathrm{OADC} / \mathrm{T}}$ supplemented with $50 \mu \mathrm{g} / \mathrm{mL}$ kanamycin. Fluorescent $M$. abscessus has been previously described (Bernut et al., 2014a).

\section{Preparation of Single Cell M. fortuitum}

Single cell preparations of fluorescent $M$. fortuitum were created, as previously described (Bernut et al., 2015). Exponential-phase M. fortuitum were pelleted at $3000 \times \mathrm{g}$ for $10 \mathrm{~min}$ at room temperature and resuspended in $1 \mathrm{~mL}$ of $7 \mathrm{H} 9^{\mathrm{OADC}} / \mathrm{T}$. Bacteria were passed through a 26 gauge needle 15 times, followed by two rounds of $10 \mathrm{~s}$ sonication, made up to $50 \mathrm{~mL}$ with $7 \mathrm{H} 9$ OADC/T and centrifuged at $3000 \times \mathrm{g}$ for $10 \mathrm{~min}$ at room temperature. Pelleted bacteria were resuspended in up to $1 \mathrm{~mL}$ of $7 \mathrm{H} 9$ OADC/T and $10 \mu \mathrm{L}$ aliquots of single cell $M$. fortuitum prepared and placed at $-80^{\circ} \mathrm{C}$ until required.

\section{Zebrafish Maintenance}

Zebrafish experiments were completed in accordance with the Comité d'Ethique pour l'Expérimentation Animale de la Région Languedoc Roussillon under the reference CEEALR36-1145. All experiments in the current study were performed using the golden mutant and macrophage reporter $\operatorname{Tg}(m p e g 1: m$ Cherry) lines as previously described (Bernut et al., 2014a, 2015). Zebrafish embryos were obtained and maintained as previously described (Bernut et al., 2015).

\section{Morpholino Injection and cftr Knockdown}

Morpholinos were designed and purchased from GeneTools. A splice-blocking morpholino specifically targeting zebrafish gene $c f t r$ (ZEBRAFISHIN, ZDB-GENE-050517-20) (5'GACACATTTTGGACACTCACACCAA-3') was injected into zebrafish embryos at the 1-4 cell stage ( $1 \mathrm{mM}, 2 \mathrm{~nL})$. Furthermore, details of the morpholino efficacy and specificity and cftr knockdown was previously validated as described (Bernut et al., 2019). Briefly, this splice blocking morpholino was designed against the exon 3-intron 3 junction within the $c f t r$ gene. Sequencing analysis indicated that the $c f t r$ morpholino blocks normal splicing, resulting in a 54 bp deletion in exon 3, and leading to the knockdown of CFTR expression (Bernut et al., 2019).

\section{Zebrafish Microinjection and Infection}

At $24 \mathrm{~h}$ post-fertilization, embryos were dechorionated using Pronase $(10 \mathrm{mg} / \mathrm{mL}$; Sigma-Aldrich) for up to $5 \mathrm{~min}$ at room temperature, followed by extensive washing in zebrafish water. Following dechorionation, embryos were injected with either liposomal clodronate or PBS-filled liposomes (Liposoma) (2 $\mathrm{nL}$ ) via caudal vein injection, as previously described (Bernut et al., 2014a, 2015). At $30 \mathrm{~h}$ post-fertilization, embryos were anesthetized in $0.02 \%$ tricaine solution and infected with fluorescent $M$. fortuitum via caudal vein injection (3 nL containing $\approx 100$ bacteria/nL). Bacterial inoculum was checked a posteriori by injection of $3 \mathrm{~nL}$ into sterile $\mathrm{PBS}$ and plating onto $7 \mathrm{H} 10^{\mathrm{OADC}}$. Following infection, embryos were transferred to 24 -well plates ( 2 embryos/well) and incubated at $28.5^{\circ} \mathrm{C}$ for the duration of the experiment. Embryo age is expressed as days post-infection (dpi).

\section{Zebrafish Monitoring and Live Imaging}

Embryo survival was monitored daily based on the presence or absence of a heartbeat. Survival curves were determined by counting dead larvae for up to 12 days, or until uninfected embryos begin to die. At designated key time points postinfection, embryos were anesthetized in $0.02 \%$ tricaine solution and mounted on $3 \%(\mathrm{w} / \mathrm{v})$ methylcellulose solution for live imaging. Images were taken using a Zeiss Axio Zoom.V16 coupled with an Axiocam 503 monochrome camera (Zeiss). Fluorescent Pixel Count (FPC) measurements were determined using the "Analyse particles" function in ImageJ. Granulomas were identified based on the co-localization of fluorescent macrophages and fluorescent $M$. fortuitum. All experiments were completed at least two times independently.

\section{Statistical Analysis}

Survival curve analysis was completed using the log-rank (Mantel-Cox) statistical test. Abscess, cord, granuloma and bacterial burden (FPC) analyses were completed using unpaired Student's t-test. All statistical tests were completed using Graphpad Prism (Version 8.0.1).

\section{RESULTS}

\section{M. fortuitum Forms Large Corded Aggregates in vitro}

Zebrafish naturally require lower ambient temperatures of $\sim 28^{\circ} \mathrm{C}$ for normal growth and development. In establishing 
A
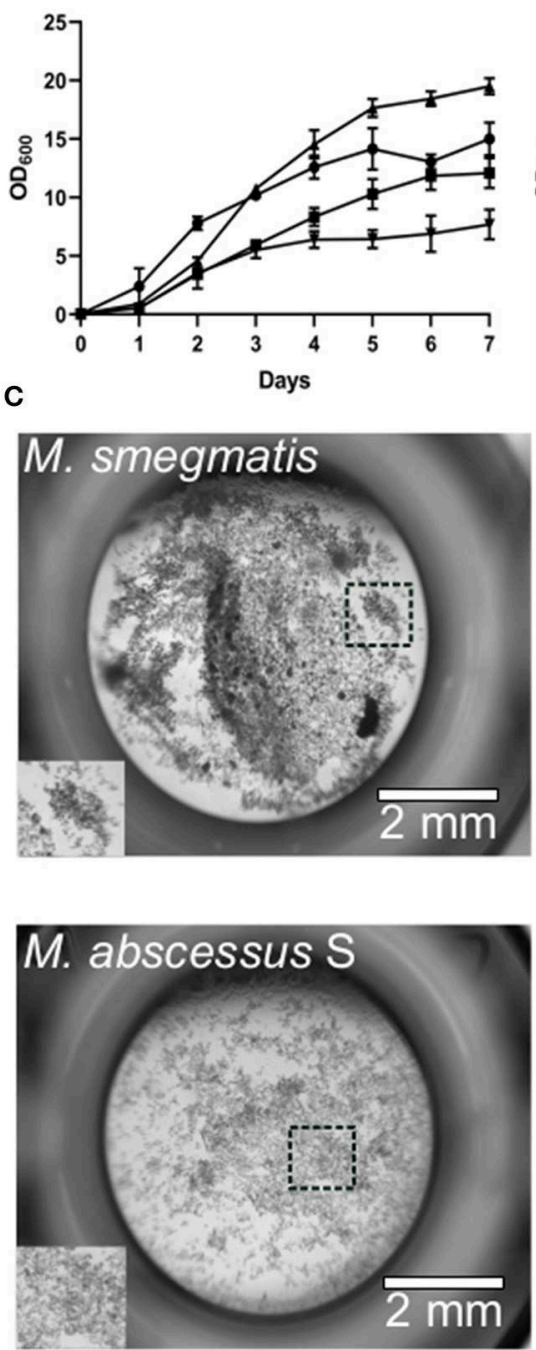

D

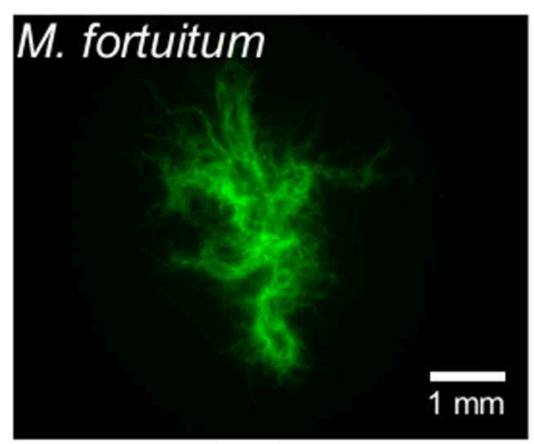

B
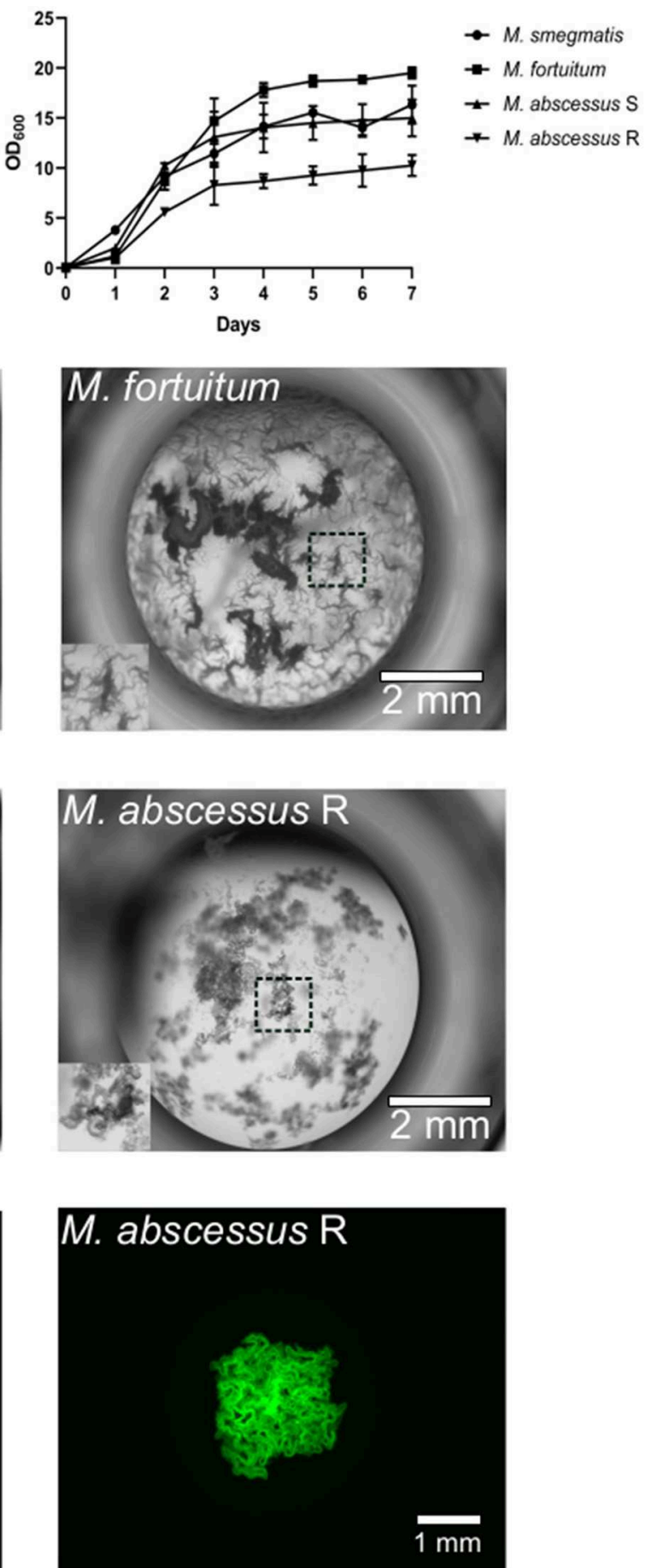

FIGURE 1 | Mycobacterium fortuitum forms large corded bacterial aggregates in vitro. (A) M. abscessus CIP104536 ${ }^{\top}$ smooth (S) and rough (R) variants, $M$. fortuitum subsp. fortuitum (ATCC 6841) and $M$. smegmatis $\mathrm{mc}^{2} 155$ were inoculated in $7 \mathrm{H} 9^{\mathrm{OADC} / \mathrm{T}}$ at an optical density of $0.05\left(\mathrm{OD}_{620}\right)$ and incubated at $30^{\circ} \mathrm{C}$ and $(\mathbf{B}) 37^{\circ} \mathrm{C}$ under shaking at $100 \mathrm{rpm}$. Growth measurements were taken daily over a 7-day period until cultures reached stationary phase. Data shown is the merge of two independent experiments. Error bars represent standard deviation. (C) In vitro liquid growth properties of the different NTM species in CaMHB medium following 3-4 days static culture at $30^{\circ} \mathrm{C}$ in a 96 -well plate. The boxed area depicts the zoomed section of liquid growth in the bottom left hand corner of each well. Scale bars represent $2 \mathrm{~mm}$. (D) In vitro solid agar growth properties of $M$. fortuitum and $M$. abscessus R variant following 3 days growth at $30^{\circ} \mathrm{C}$. Scale bars represent $1 \mathrm{~mm}$. 
A

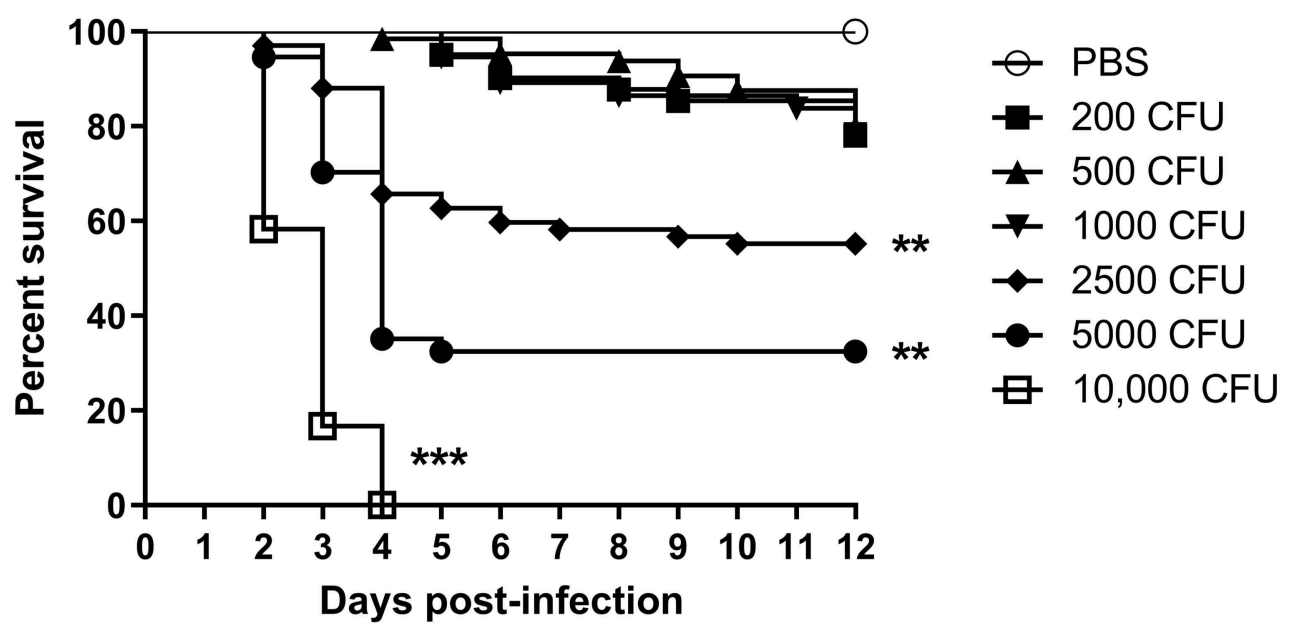

B
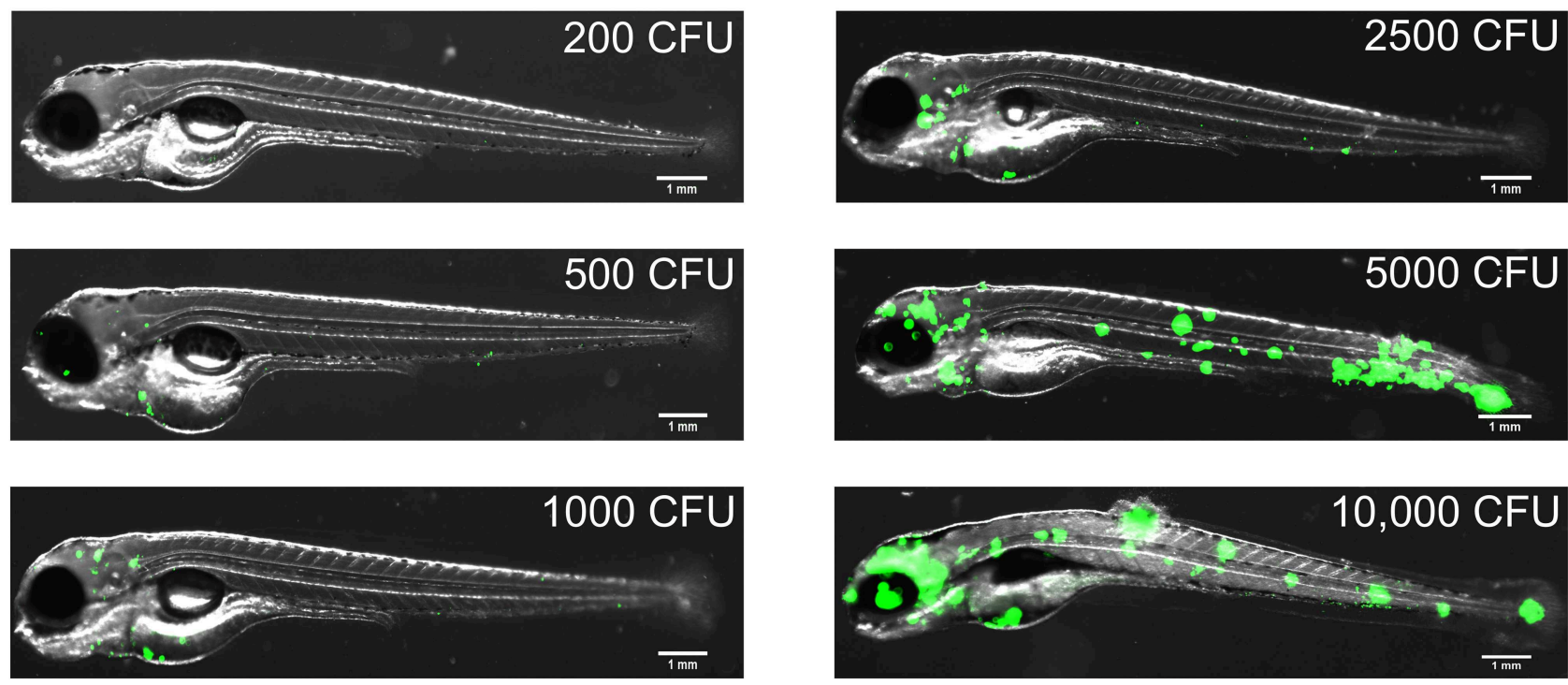

FIGURE 2 | Zebrafish embryos are susceptible to Mycobacterium fortuitum infection in a dose-dependent manner. (A) Zebrafish embryos were injected with varying dosages of GFP-expressing M. fortuitum at $30 \mathrm{~h}$ post-fertilization via caudal vein injection. Embryo survival was monitored over a 12 day period. $\mathrm{N}=20-25$ embryos/group. Statistical analysis was completed using the log-rank (Mantel-Cox) statistical test for survival curves. Data shown is the merge of two independent experiments. (B) Representative images of zebrafish embryos infected with varying dosages of GFP-expressing $M$. fortuitum at 2 days post-infection. Green represents $M$. fortuitum. Scale bars represent $1 \mathrm{~mm}$. ${ }^{\star \star} P \leq 0.01,{ }^{* \star *} P \leq 0.001$.

a new model to study the pathogenesis of a disease-causing agent, it is critical that the replication of the pathogen is not affected by these temperatures. As has been reported for other NTM such as M. abscessus (Bernut et al., 2014a) and M. kansasii (Johansen and Kremer, 2020), these species are able to replicate at lower ambient temperatures, albeit at a slower rate, highlighting their adaptability to the zebrafish platform. We firstly wanted to determine whether $M$. fortuitum is able to grow at lower temperatures compared to traditional $37^{\circ} \mathrm{C}$ incubation. When grown in $7 \mathrm{H} 9^{\mathrm{OADC} / \mathrm{T}}$ at $30^{\circ} \mathrm{C}$, we observed that $M$. fortuitum was able to reach early stationary phase around Day six, which was comparable to other rapid growers that are known to thrive at lower temperatures, such as M. abscessus and M. smegmatis (Figure 1A). Comparatively, all species grew at a much faster rate and reached stationary phase earlier when grown at $37^{\circ} \mathrm{C}$, which was to be expected and has been reported for other NTM species (Johansen and Kremer, 2020) (Figure 1B).

It has been well-described that specific mycobacteria are able to grow in large cord-like aggregates; a trait that has 
A

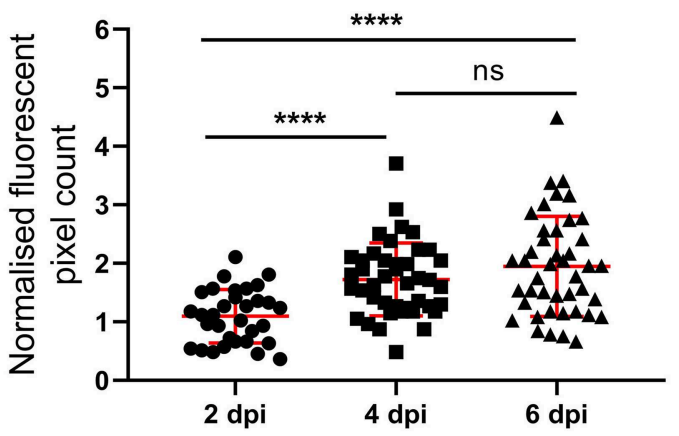

B

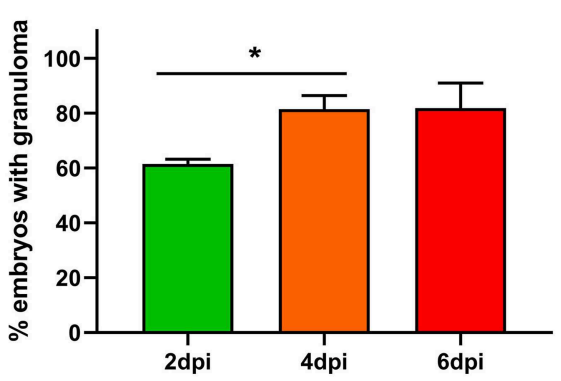

D

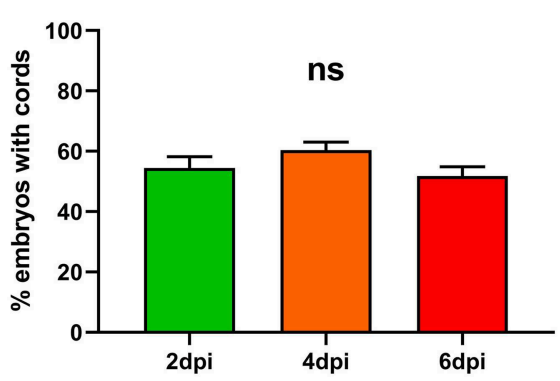

$\mathbf{F}$

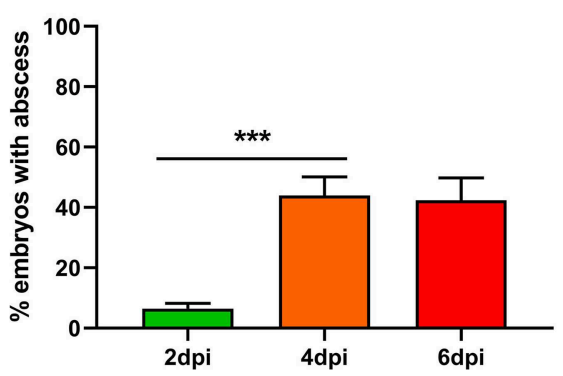

C

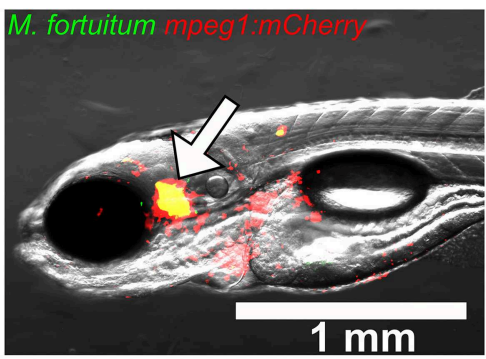

E

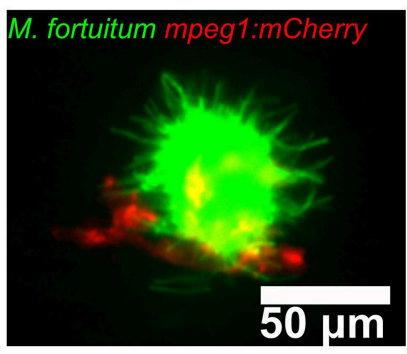

G

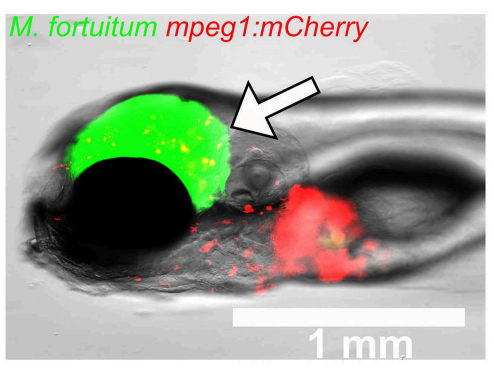

FIGURE 3 | Zebrafish infected with Mycobacterium fortuitum recapitulate the hallmark features of other pathogenic mycobacteria. (A) Following infection mpeg1::mCherry zebrafish embryo at $30 \mathrm{~h}$ post-fertilization via caudal vein injection with $300 \mathrm{CFU}$ of GFP-expressing M. fortuitum, bacterial burden was quantified using fluorescent pixel count (FPC determination using ImageJ software) at 2, 4, and 6 days post-infection. For each experiment, all groups were normalized against the 2 days post-infection group. Each datapoint represents an individual embryo. Error bars represent standard deviation. Statistical significance was determined by Student's $t$-test. Plots represent a pool of 2 independent experiments containing approximately 20 embryos per group. (B) The granuloma formation kinetic in embryos following infection with M. fortuitum. Error bars represent standard deviation. Data shown is the merge of two independent experiments. Statistical significance was determined by Student's $t$-test. (C) Representative image of a granuloma at 4 days post-infection. The white arrow highlights the granuloma. Scale bar represents $1 \mathrm{~mm}$. (D) The kinetic of bacterial cord formation in zebrafish embryos following M. fortuitum infection. Error bars represent standard deviation. Data shown is the merge of two independent experiments. Statistical significance was determined by Student's $t$-test. (E) Representative image of a bacterial corded M. fortuitum aggregate surrounded by recruited macrophages at 4 days post-infection. Note the sheer size of the bacterial aggregate in comparison to the smaller macrophages. Green represents M. fortuitum, while red represents macrophages. Scale bar represents $50 \mu \mathrm{m}$. (F) The kinetic of abscess formation in zebrafish embryos infected with $M$. fortuitum. Error bars represent standard deviation. Data shown is the merge of two independent experiments. Statistical significance was determined by Student's $t$-test. (G) Representative image of an $M$. fortuitum abscess at 4 days post-infection. The white arrow highlights the abscess. Green represents M. fortuitum, while red represents macrophages. Scale bar represents $1 \mathrm{~mm} .{ }^{\star} P \leq 0.05,{ }^{\star \star \star *} P \leq 0.001,{ }^{\star \star \star \star} P \leq 0.0001$. 
A

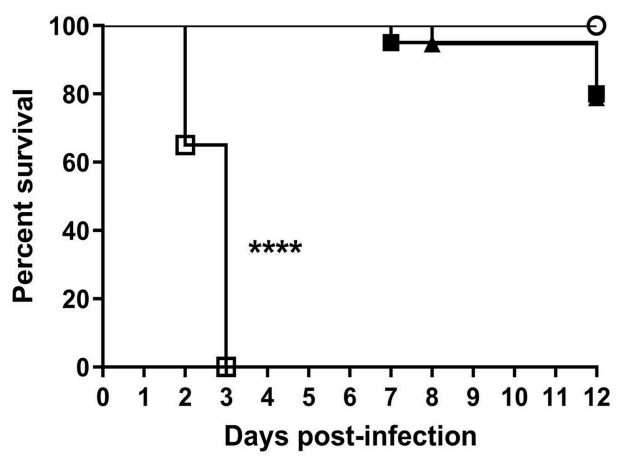

C

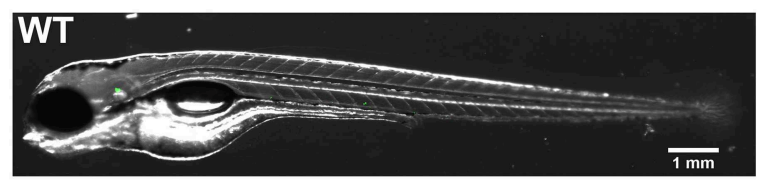

D

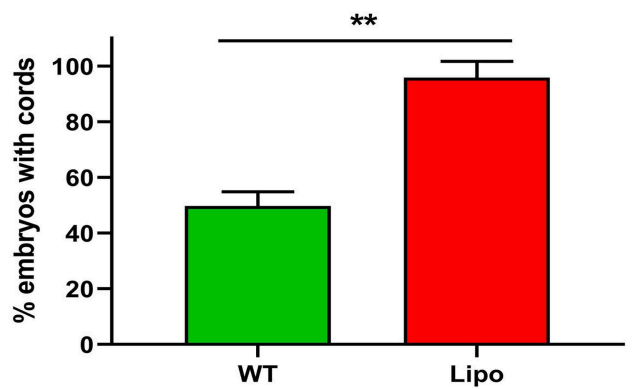

E

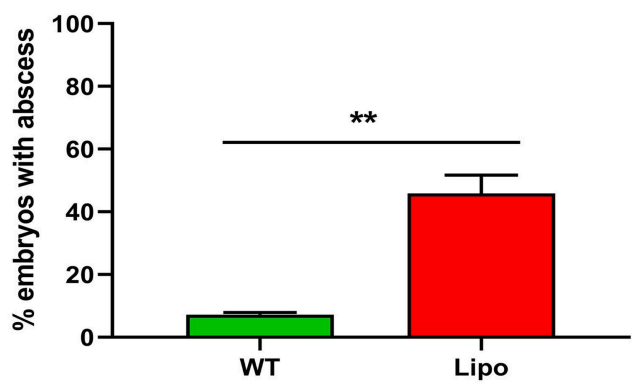

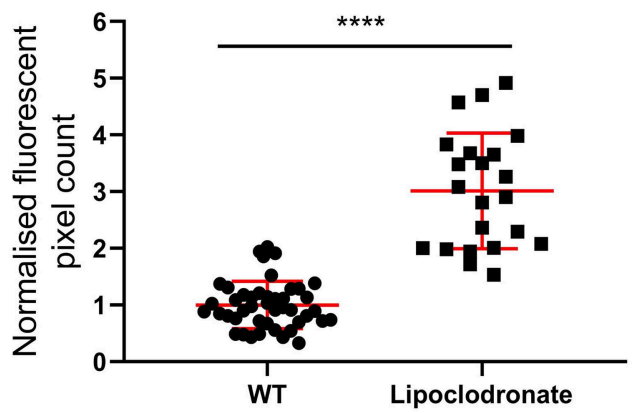
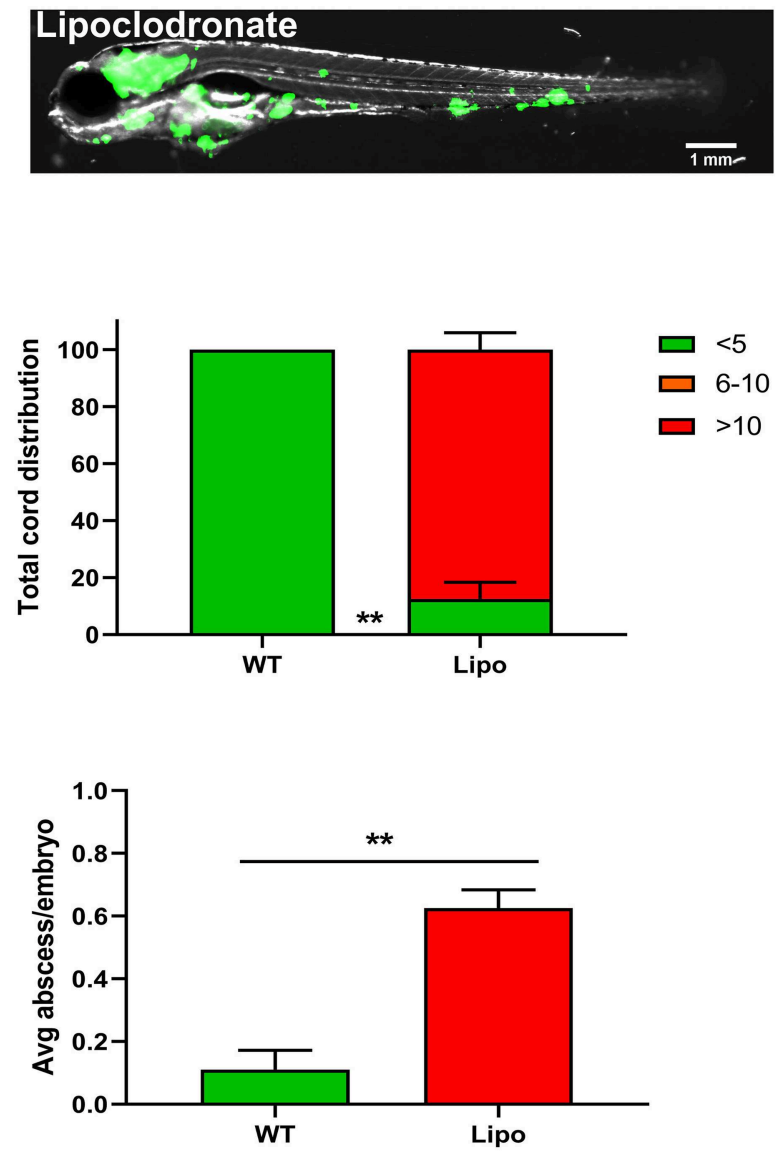

FIGURE 4 | Lipoclodronate macrophage depletion results in lethal M. fortuitum infection. (A) At $24 \mathrm{~h}$ post-fertilization, embryos were treated with liposomal clodronate via caudal vein injection to transiently deplete macrophages. At $30 \mathrm{~h}$ post-fertilization, embryos were injected intravenously with $300 \mathrm{CFU}$ of GFP-expressing M. fortuitum with embryo survival monitored over a 12 day period. $n=20-25$ embryos/group. Statistical analysis was completed using the log-rank (Mantel-Cox) statistical test for survival curves. Data shown is the merge of two independent experiments. (B) Bacterial burden at 2 days post-infection was calculated using fluorescent pixel count (FPC) determination with ImageJ software. Lipoclodronate-treated embryos were normalized against corresponding controls in each experiment. Each datapoint represents an individual embryo. Error bars represent standard deviation. Statistical significance was determined by Student's $t$-test. Plots represent a pool of 2 independent experiments containing approximately 20 embryos per group. (C) Representative images of wild-type (WT) and Lipoclodronate-treated embryos infected with 300 CFU of GFP-expressing M. fortuitum at 2 days post-infection. Scale bars represent 1 mm. (D) The proportion of embryos with bacterial cords and the total distribution of cords categorized as low ( $<5$ cords/embryo), moderate (6-10 cords/embryo) and high ( $>10$ cords/embryo) in $M$. fortuitum-infected embryo at 2 days post-infection. Error bars represent standard deviation. Data shown is the merge of two independent experiments. Statistical significance was determined by Student's t-test. (E) The proportion of embryos with abscesses and the average number of abscesses per infected embryo at 2 days post-infection. Error bars represent standard deviation. Data shown is the merge of two independent experiments. Statistical significance was determined by Student's $t$-test. ${ }^{* \star} P \leq 0.01,{ }^{\star \star \star *} P \leq 0.0001$. 
A

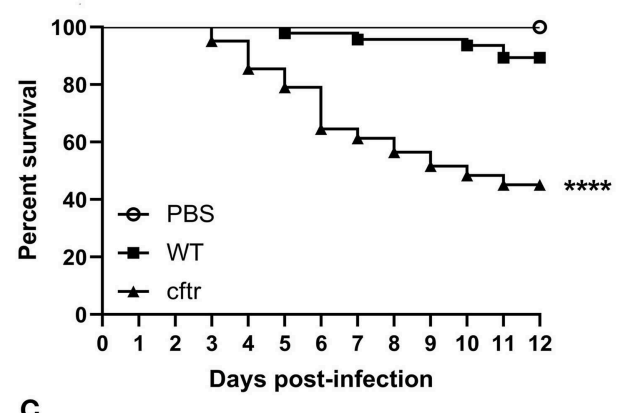

C

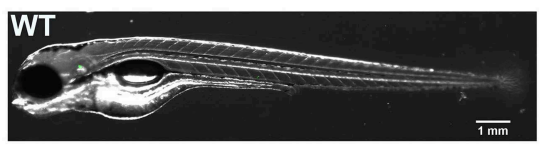

D
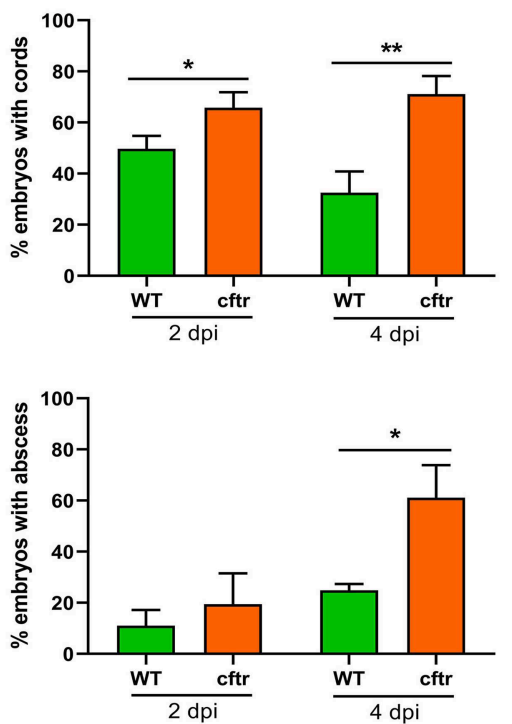

$\mathbf{F}$

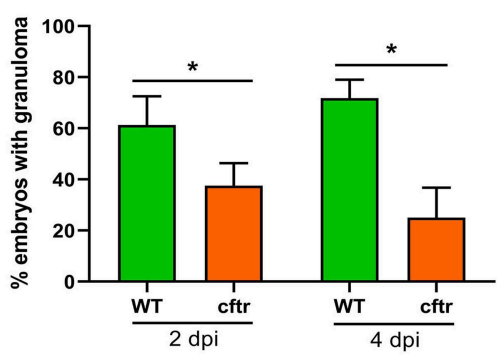

B
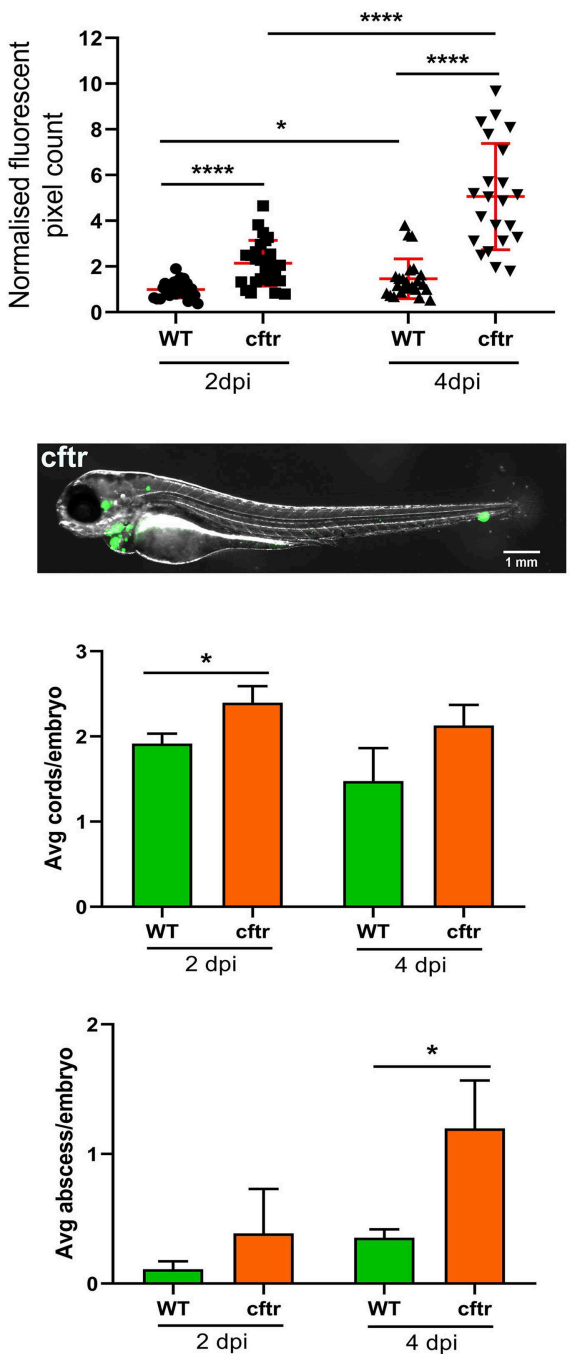

ns

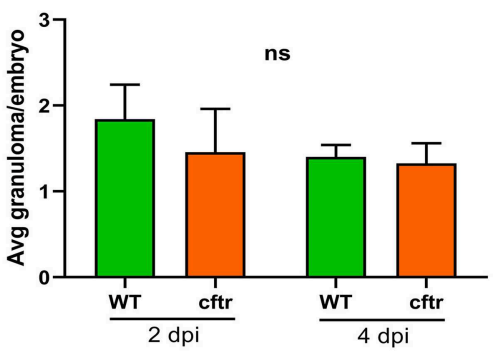

FIGURE 5 | CFTR ablation leads to rapid larval death and uncontrolled bacterial expansion. (A) Cftr mrophants at $30 \mathrm{~h}$ post-fertilization were infected with $300 \mathrm{CFU}$ of GFP-expressing M. fortuitum via caudal vein injection and embryo survival was monitored over a 12 day period. $N=20-25$ embryos/group. Statistical analysis was completed using the log-rank (Mantel-Cox) statistical test for survival curves. Data shown is the merge of three independent experiments. (B) Bacterial burden was quantified by fluorescent pixel count (FPC) determination using Image J software, with each data point representing an individual embryo. Each group was normalized against the wild-type cohort at 2 days post-infection. Error bars represent standard deviation. Statistical significance was determined by Student's $t$-test. Plots represent a pool of three independent experiments containing 20-25 embryos per group. (C) Representative images of wild-type (WT) and CFTR depleted (cftr) embryos infected with 300 CFU of GFP-expressing M. fortuitum at 2 days post-infection. Scale bars represent $1 \mathrm{~mm}$. (D) The proportion of embryos with bacterial cords and the average number of cords per infected embryo at 2 and 4-days post-infection. Error bars represent standard deviation. Data shown is the merge of three independent experiments. Statistical significance was determined by Student's $t$-test. (E) The proportion of embryos with abscesses and the average number of abscesses per infected embryo at 2 and 4-days post-infection. Error bars represent standard deviation. Data shown is the merge of three independent experiments. Statistical significance was determined by Student's $t$-test. $(\mathbf{F})$ The proportion of embryos with granulomas and the average number of granulomas per infected embryo at 2 and 4-days post-infection. Error bars represent standard deviation. Data shown is the merge of three independent experiments. Statistical significance was determined by Student's $t$-test. ${ }^{\star} P \leq 0.05,{ }^{\star *} P \leq 0.01,{ }^{* \star \star *} P \leq 0.0001$. 


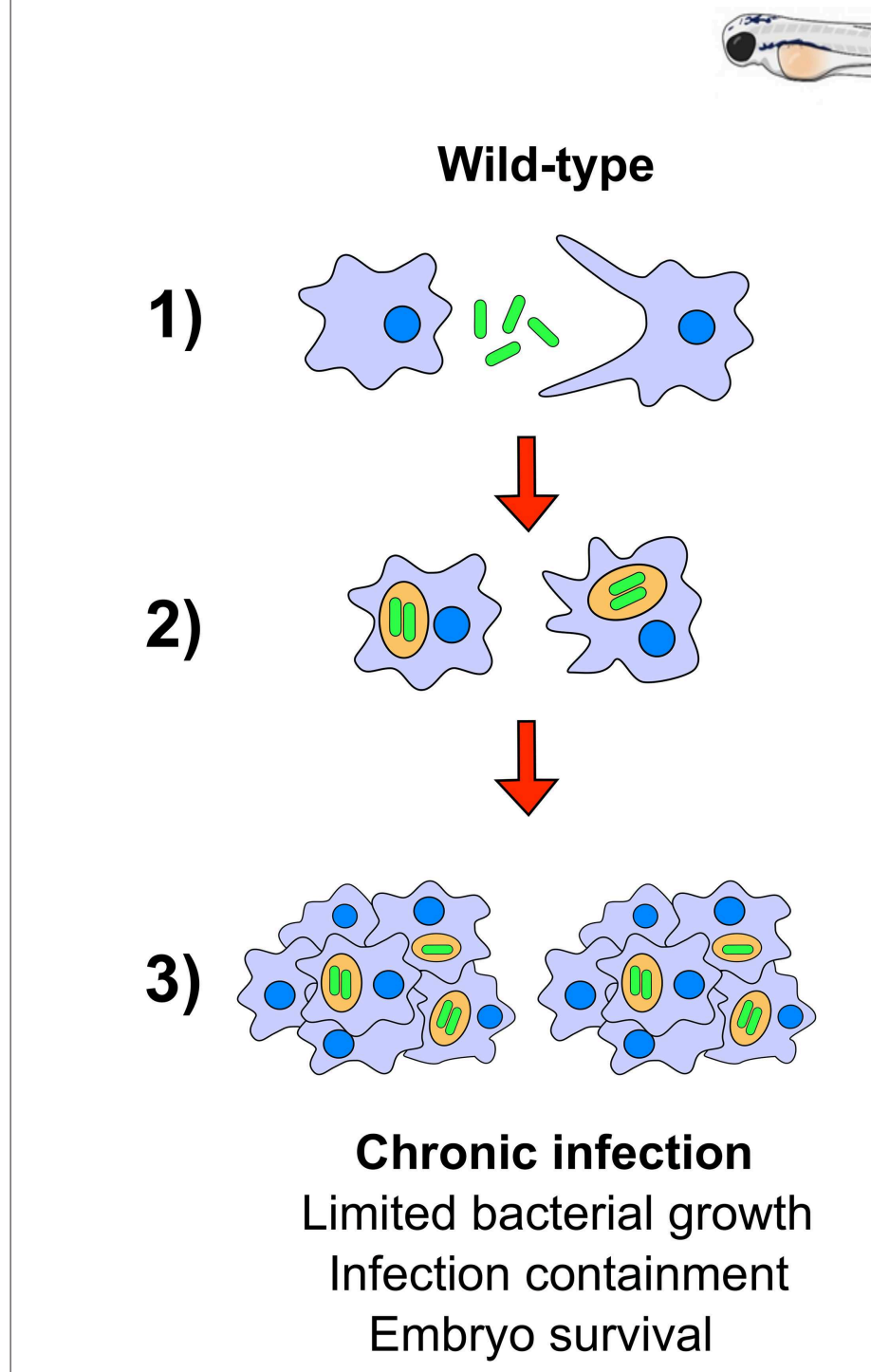

CFTR depletion
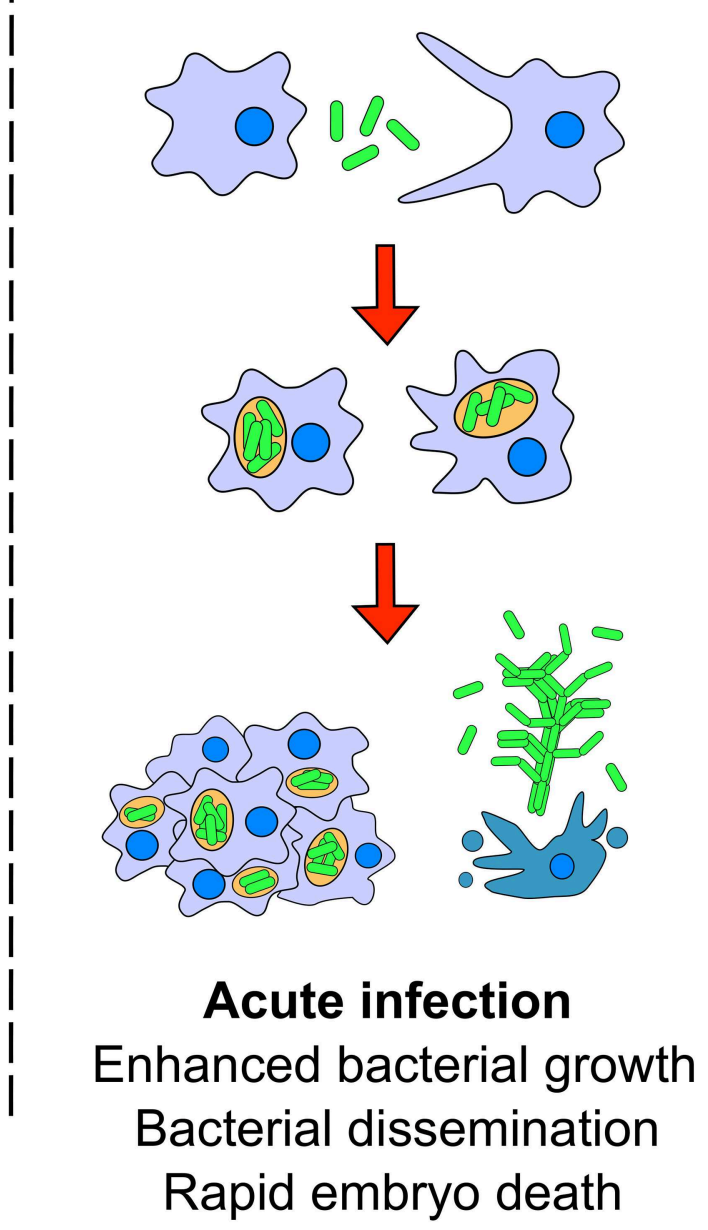

FIGURE 6 | A hypothetical schematic summarizing the pathogenesis of Mycobacterium fortuitum in both wild-type and CFTR backgrounds. (1) Following intravenous injection of $M$. fortuitum into zebrafish embryos, macrophages are rapidly recruited to the site of infection and phagocytose bacilli; (2) Once inside the phagosome, $M$. fortuitum is able to initially replicate, however in a wild-type scenario, host defenses are able to slow the bacterial expansion until further macrophages are recruited. Comparatively, following CFTR depletion, bacteria are able to rapidly expand within the phagosome, presumably due to defective host oxidative defense mechanisms. (3) Additional macrophages are recruited to the infection foci, forming the granuloma which acts to contain infection dissemination in wild-type embryos. In a proportion of wild-type embryos, we propose that macrophage apoptosis triggers the escape of bacilli to the extracellular space which facilitates the growth of bacilli into large corded aggregates, promoting zebrafish embryo death. In CFTR-depleted embryos, there are fewer granulomas and a greater proportion of cords which leads to more rapid embryonic death and a greater proportion of cords and abscesses.

been often regarded for the most pathogenic mycobacteria such as Mycobacterium tuberculosis, M. marinum, M. abscessus and recently M. kansasii (Glickman et al., 2000; Staropoli and Branda, 2008; Bernut et al., 2014a, 2016b; Johansen and Kremer, 2020). As such, we wanted to determine whether $M$. fortuitum also possessed similar traits, which may explain why it is regarded as an opportunistic pathogen and frequently isolated in pulmonary and extra-pulmonary sites (Wallace et al., 1983; Brown-Elliott and Philley, 2017). Remarkably, when grown at both 30 and $37^{\circ} \mathrm{C}$ in $\mathrm{CaMHB}$, a liquid medium commonly used for testing of antimicrobial susceptibility, we observed the formation of large cord-like aggregates for $M$. fortuitum (Figure 1C). These cords were different in size and appearance when compared to rough $M$. abscessus; an NTM species wellknown for its large extracellular cord formation both in vitro and in vivo (Bernut et al., 2014a, 2016a,b). When we observed bacterial growth on $7 \mathrm{H} 10^{\mathrm{OADC}}$ solid agar, we also identified the presence of large cord-like structures for $M$. fortuitum, which were noticeably different in appearance to rough $M$. abscessus (Figure 1D). 


\section{M. fortuitum Is Pathogenic in a Zebrafish Model in a Dose-Dependent Manner}

We firstly wanted to determine whether $M$. fortuitum was able to promote embryonic death in a dose-dependent manner. At $30 \mathrm{~h}$ post-fertilization, bacterial suspensions of $M$. fortuitum were injected in the caudal vein of zebrafish embryos. We observed that embryos infected with 200, 500 or 1,000 CFU of $M$. fortuitum induced $\sim 20 \%$ embryonic death in a 12 day period (Figure 2A). This is similar to what has been observed for other NTM, such as the smooth variant of M. abscessus (Bernut et al., 2014a) or M. kansasii (Johansen and Kremer, 2020). Moreover, infection with higher doses, corresponding to 2,500 or $5,000 \mathrm{CFU}$, led to 40 and $70 \%$ of embryo death, respectively, within a 12-day period. Interestingly, larval killing occurred rapidly (within 4 days post-infection) after which the percentage of survival remained stable. Embryos infected with 10,000 CFU all succumbed within a 4-day period. Whole embryo imaging demonstrates that larval killing was directly correlated to the bacterial burden and that bacteria were disseminated throughout the entire embryo, suggestive of a systemic infection (Figure 2B). Collectively, these findings demonstrate the feasibility of the zebrafish model to study acute infections with $M$. fortuitum.

\section{The Zebrafish/M. fortuitum Model Recapitulates Important Mycobacterial Pathophysiological Features}

We next monitored the kinetic of the bacterial burden upon intravenous infection of embryos with $300 \mathrm{CFU}$ of $M$. fortuitum expressing GFP by determination of fluorescent pixel counts (FPC). There was a significant increase in the bacterial loads between 2 and $4 \mathrm{dpi}$, after which bacterial loads remained stable between 4 and $6 \mathrm{dpi}$, suggesting that the bacterial expansion is controlled at later time points (Figure 3A).

A major feature of mycobacterial infection is the formation of the granuloma (Pagán and Ramakrishnan, 2018). Importantly, zebrafish embryos do not possess adaptive immunity and so granuloma identification is strictly based on the presence of macrophages at the infection foci (Davis et al., 2002). This host immune aggregate acts to restrict pathogen spread, however mycobacteria have developed unique mechanisms allowing their prolonged survival within these host structures (Volkman et al., 2004; Davis and Ramakrishnan, 2009; Bernut et al., 2014a; Johansen and Kremer, 2020). Comparatively, extracellular cord formation within the zebrafish embryo is often representative of acute infection and generally is indicative of mycobacterial escape from the phagosome and macrophage destruction (Bernut et al., 2014a, 2015, 2016a). Abscess formation often represents loss of infection control and typically occurs following extracellular cord formation and expansion (Bernut et al., 2014a, 2015, 2016a). Abscesses represent a marker of disease severity and are often associated with cellular debris, tissue destruction, and acute infection in zebrafish (Bernut et al., 2014a). As such, we wanted to determine whether zebrafish embryos infected with M. fortuitum are able to recapitulate critical determinants of mycobacterial infection. To visualize and monitor the presence of granulomas, infections with $M$. fortuitum expressing GFP were carried out in the caudal vein of the mpeg1:mCherry zebrafish reporter line harboring red fluorescent macrophages. We observed the presence of granulomas in approximately $60 \%$ of infected embryos at $2 \mathrm{dpi}$, which increased to $80 \%$ of embryos by $4 \mathrm{dpi}$ and then remained constant between 4 and 6 dpi (Figures 3B,C). This coincides with the arrest of bacterial proliferation between 4 and $6 \mathrm{dpi}$ as seen in Figure 3A. When we counted the number of embryos with cords, $\sim 50 \%$ of embryos displayed these extracellular bacterial structures, which was consistent throughout the experiment until 6 dpi (Figures 3D,E). We observed a significant increase in the number of embryos with abscesses from 5 to $40 \%$ between 2 and 4 dpi (Figures 3F,G). These findings demonstrate that the zebrafish model effectively recapitulates critical aspects of mycobacterial pathophysiology following $M$. fortuitum infection and highlights the applicability of this model for the study of $M$. fortuitum pathogenesis. These results also suggest that acute infection is typified by rapid bacterial expansion and that cord formation occurs very rapidly within $2 \mathrm{dpi}$, while the bacterial growth is controlled once granulomas are fully formed at 4 dpi.

\section{Macrophages Are Essential Host Determinants of $M$. fortuitum Infection Control}

Macrophages are the first responders to infection and one of the major cell populations within the mycobacterial granuloma (Volkman et al., 2004; Bernut et al., 2016a; Pagán and Ramakrishnan, 2018). It is well-known that macrophage depletion has a detrimental outcome for mycobacterial infection in zebrafish embryos, leading to premature larval death within several days post-infection (Clay et al., 2007; Bernut et al., 2014a). Currently, it is not known whether macrophages play such a crucial role in M. fortuitum infection as compared to other mycobacterial species. As such, we aimed to elucidate whether macrophages are essential determinants of $M$. fortuitum infection control. Liposomal clodronate is highly effective in depleting macrophages within the zebrafish embryo for up to 6 days as previously reported (Bernut et al., 2014a, 2015). Using Lipoclodronate macrophage depletion, $100 \%$ killing was achieved in animals infected with $\approx 300 \mathrm{CFU}$ at $3 \mathrm{dpi}$ (Figure 4A). This was associated with a massive increase in bacterial loads in macrophage-depleted embryos as compared to wild-type fish receiving PBS clodronate at $2 \mathrm{dpi}$, thus highlighting the critical role of macrophages in controlling $M$. fortuitum infection (Figure 4B). Further, embryo imaging clearly highlighted the very high bacterial loads in the macrophage-depleted embryos as compared to the wild-type fish (Figure 4C), consistent with bacterial burden quantification. Importantly, we observed a greater proportion of embryos with cords and an increase in the number of cords per embryo in Lipoclodronate-treated embryos as compared to PBS-injected animals, emphasizing the need of macrophages to restrict intracellular growth and preventing extracellular cord formation (Figure 4D). There 
was also a greater proportion of Lipoclodronate treated embryos with abscesses and a higher number of abscesses per embryo when compared to PBS groups at 2 dpi (Figure 4E). Together, these results demonstrate that the hypersusceptibility of Lipoclodronate-treated embryos to $M$. fortuitum stems from their lack of macrophages.

\section{CFTR Depletion Leads to Increased Susceptibility to $M$. fortuitum Infection}

It is estimated that $5-20 \%$ of CF patients will develop NTM infection (Richards and Olivier, 2019), indicating that these patients are at increased risk of infection due to genetic susceptibility. Although less frequently encountered than $M$. avium or $M$. abscessus, several studies have reported the presence of $M$. fortuitum in CF patients (Sermet-Gaudelus et al., 2003; Cândido et al., 2014; Martiniano et al., 2016; Richards and Olivier, 2019). Recent work has shown that CFTR is important for fine-tuning host oxidative stress and restricting intracellular growth of $M$. abscessus in a zebrafish model (Bernut et al., 2019). These embryos also possessed fewer granulomas and granulomas displayed a disorganized structure, suggesting that CFTR is also an important determinant of infection containment within the granuloma microenvironment. We thus inquired whether CFTR depletion also conferred increased susceptibility to $M$. fortuitum infection. Using a morpholino knockdown strategy previously validated to target the zebrafish cftr gene (Bernut et al., 2019), we observed a massive increase in embryo mortality (almost 60\% mortality at $12 \mathrm{dpi}$ ) following $M$. fortuitum infection in cftr morphants when compared to wild-type infected embryos (Figure 5A). In addition, mortality started earlier in $c f t r$ morphants than in wild-type fish. When we further examined bacterial burden, we observed an increase in CFTR-depleted embryos at 2 dpi when compared to wild-type infection, which was greatly increased by 4 dpi (Figures 5B,C). Strikingly, in CFTR-depleted embryos, there was an enhanced proportion of embryos with cords at both 2 and 4 dpi when compared to wild-type infection. Moreover, there was an increase in the number of cords per embryo in CFTR-depleted embryos at 2 dpi when compared to wildtype (Figure 5D). There was also a significantly greater number of embryos with abscesses and a greater number of abscesses per embryo at $4 \mathrm{dpi}$ in the CFTR-depleted group as compared to wild-type infections (Figure 5E). In contrast, infected cftr morphants produced less granulomas when compared to wild-type infections, however the number of granulomas per embryo remained constant over time (Figure 5F), similarly to what was previous observed in M. abscessus infected cftr morphants (Bernut et al., 2019). In contrast to wild-type embryos where bacterial proliferation is constrained when granulomas are formed (Figure 3), the opposite scenario occurs in cftr morphants whereby bacterial proliferation progresses whilst the number of granulomas remains unchanged. These findings suggest that depletion of CFTR impairs host immune responses resulting in granuloma formation.

Overall, these findings emphasize the importance of CFTR as a host factor in controlling $M$. fortuitum pathogenesis through granuloma formation and preventing extracellular cord formation, indicating that CFTR dysfunction results in hypersusceptibility to $M$. fortuitum infection.

\section{DISCUSSION}

Mycobacterium fortuitum is increasingly recognized as an opportunistic nosocomial pathogen responsible for a wide panel of clinical infections, including lung diseases. However, our understanding of the pathogenic mechanisms of this important NTM species remains obscured by the lack of relevant animal models. Herein, we exploited the zebrafish model to directly and non-invasively dissect critical steps in the pathogenesis of M. fortuitum. Genetic manipulation of the host, combined with a fluorescent reporter bacterial strain were used to decipher the interplay between $M$. fortuitum and host macrophages. We provide evidence that zebrafish embryos, displaying only innate immunity, represent a suitable and permissive host that is susceptible to high doses of $M$. fortuitum. Chemical depletion of macrophages led to a rapid expansion of $M$. fortuitum, establishing a systemic and acute infection leading to rapid larval killing. Most importantly, morpholino depletion of CFTR led to hypersusceptibility of zebrafish embryos to M. fortuitum infection, providing the first insight into the significance of CFTR in $M$. fortuitum pathogenesis. The pathophysiological events of $M$. fortuitum infection are reminiscent to those reported earlier for M. abscessus, a well-acknowledged CF pathogen and can be summarized in a sequential manner (Figure 6): (1) Following intravenous injection, $M$. fortuitum is rapidly phagocytosed by macrophages; (2) Once inside the phagosome, M. fortuitum is initially able to replicate until the recruitment of additional macrophages to the site of infection; (3) Infection is contained with the formation of the mycobacterial granuloma and leads to a chronic infection. However, in a subset of wild-type embryos and to a much greater extent in CFTR embryos, it is very likely that the escape or release of $M$. fortuitum from infected macrophages is favoring extracellular bacterial multiplication in the form or serpentine cords (highly stimulated in macrophagedepleted embryos). Bacterial cords can withstand phagocytosis by macrophages which further exacerbates uncontrolled bacterial multiplication and ultimately results in abscess formation, tissue damage and larval death. This is supported by recent findings indicating that caspase-mediated apoptosis of catfish macrophages occurs rapidly after infection with $M$. fortuitum (Datta et al., 2018). Thus, one can envisage a similar scenario where apoptosis of zebrafish macrophages releases $M$. fortuitum into the extracellular milieu, promoting the production of extracellular cords.

Multiple hypotheses may explain why high doses of $M$. fortuitum are required to induce efficient and rapid killing of zebrafish larvae, as compared to M. marinum (Volkman et al., 2004; Davis and Ramakrishnan, 2009). One plausible explanation could be attributed to the fact that $M$. fortuitum remains phagolysosomal, presumably because of the lack of ESAT-6 secretion (Houben et al., 2012), although pan-genomic analyses seem to indicate that the $M$. fortuitum genome possesses an 
ESX-1 cluster (Dumas et al., 2016). Indeed, a clear link between translocation and mycobacterial virulence has been proposed, relying on a functional ESX-1 system and ESAT-6 secretion, which facilitates translocation of members of the $M$. tuberculosis complex and $M$. marinum from the phagolysosome to the cytosol (Houben et al., 2012). However, M. abscessus which escapes the phagosome lacks ESX-1, but it has been postulated that communication with the cytosolic compartment is favored by the presence of a functional ESX-4 secretion system (Roux et al., 2016; Laencina et al., 2018; Johansen et al., 2020). Future work is therefore required to investigate the contribution of the different ESX systems in the pathogenicity of M. fortuitum.

Moreover, we have harnessed the CFTR zebrafish model of infection as an innovative vertebrate recapitulating aspects of CF immuno-pathogenesis (Bernut et al., 2019). M. fortuituminfected CFTR-depleted zebrafish rapidly succumbed to infection, reflecting a hypersusceptibility phenotype to this mycobacterial species in CF and providing a first glance into the vulnerability of CF patients to $M$. fortuitum infection. Previous work indicated CFTR participates in neutrophil chemotaxis to the infected sites and the adjustment of oxidative host defenses, conditioning efficient phagocyte-mediated bacterial killing, together generating a protective granulomatous response (Bernut et al., 2019). However, the role of neutrophils in the defense against $M$. fortuitum remains to be investigated in future studies. CFTR depletion is also associated with a deficiency in radical oxygen species production altering phagocyte-mediated killing. It is, therefore, very likely that this low oxidative response in $c f t r$ morphants results in increased intracellular loads of M. fortuitum and premature cell death. Thus, the reduced number of protective granulomas together with the uncontrolled extracellular mycobacterial spread leads to acute infection and larval death in $\mathrm{ctr}$ morphants. Our study indicates that CFTR is a regulator of host immunity to $M$. fortuitum, as suggested previously for $M$. abscessus. Importantly, CFTR depletion has been shown to have no impact on the control of host immunity to the non-pathogenic $M$. smegmatis nor to the pathogenic M. marinum (Bernut et al., 2019). These findings imply that species-specific restriction mechanisms occur for various NTM,

\section{REFERENCES}

Adams, K. N., Takaki, K., Connolly, L. E., Wiedenhoft, H., Winglee, K., Humbert, O., et al. (2011). Drug tolerance in replicating mycobacteria mediated by a macrophage-induced efflux mechanism. Cell 145, 39-53. doi: 10.1016/j.cell.2011.02.022

Alibaud, L., Rombouts, Y., Trivelli, X., Burguière, A., Cirillo, S. L. G., Cirillo, J. D., et al. (2011). A Mycobacterium marinum TesA mutant defective for major cell wall-associated lipids is highly attenuated in Dictyostelium discoideum and zebrafish embryos. Mol. Microbiol. 80, 919-934. doi: 10.1111/j.1365-2958.2011.07618.x

Berg, R. D., and Ramakrishnan, L. (2012). Insights into tuberculosis from the zebrafish model. Trends Mol. Med. 18, 689-690. doi: 10.1016/j.molmed.2012.10.002

Bernut, A., Dupont, C., Ogryzko, N. V., Neyret, A., Herrmann, J.-L., Floto, R. A., et al. (2019). CFTR protects against Mycobacterium abscessus an interesting observation that will require further investigation. Overall, this new animal model allows us to propose that $c f t r$ represents a gene of susceptibility to $M$. fortuitum infection.

This study also paves the way to new translational possibilities in the fight against $M$. fortuitum infections. The zebrafish model described here, particularly conducive to spatiotemporal imaging of $M$. fortuitum infections, may also be exploited to test the in vivo efficacy of known antibiotics or new drug treatments. It may represent a unique biological system allowing non-invasive observations to evaluate, in real time, the efficacy of compounds in a living vertebrate, as shown previously for $M$. marinum (Takaki et al., 2012) and M. abscessus (Bernut et al., 2014b; Dubée et al., 2015; Dupont et al., 2017; Raynaud et al., 2019) and applied to high-throughput in vivo testing of drug efficacy (Carvalho et al., 2011).

\section{DATA AVAILABILITY STATEMENT}

The raw data supporting the conclusions of this article will be made available by the authors, without undue reservation, to any qualified researcher.

\section{ETHICS STATEMENT}

The animal study was reviewed and approved the Comite d'Ethique pour l'Expérimentation Animale de la Région Languedoc Roussillon under the reference CEEALR36-1145.

\section{AUTHOR CONTRIBUTIONS}

MJ conducted experiments, analyzed the data, and wrote the paper. LK conceived the idea of the project, analyzed the data, and wrote the paper. All authors contributed to the article and approved the submitted version.

\section{FUNDING}

MJ received a post-doctoral fellowship granted by Labex EpiGenMed, an Investissements d'avenir program (ANR-10LABX-12-01). doi: 10.1016/j.celrep.2019.01.071

Bernut, A., Dupont, C., Sahuquet, A., Herrmann, J.-L., Lutfalla, G., and Kremer, L. (2015). Deciphering and imaging pathogenesis and cording of Mycobacterium abscessus in zebrafish embryos. J. Vis. Exp. 103:e53130. doi: 10.3791/ 53130

Bernut, A., Herrmann, J.-L., Kissa, K., Dubremetz, J.-F., Gaillard, J.-L., Lutfalla, G., et al. (2014a). Mycobacterium abscessus cording prevents phagocytosis and promotes abscess formation. Proc. Natl. Acad. Sci. U.S.A. 111, E943-E952. doi: $10.1073 /$ pnas.1321390111

Bernut, A., Le Moigne, V., Lesne, T., Lutfalla, G., Herrmann, J.-L., and Kremer, L. (2014b). In vivo assessment of drug efficacy against Mycobacterium abscessus using the embryonic zebrafish test system. Antimicrob. Agents Chemother. 58, 4054-4063. doi: 10.1128/AAC.00142-14

Bernut, A., Nguyen-Chi, M., Halloum, I., Herrmann, J.-L., Lutfalla, G., and Kremer, L. (2016a). Mycobacterium abscessus-induced granuloma formation is 
strictly dependent on TNF signaling and neutrophil trafficking. PLoS Pathog. 12:e1005986. doi: 10.1371/journal.ppat.1005986

Bernut, A., Viljoen, A., Dupont, C., Sapriel, G., Blaise, M., Bouchier, C., et al. (2016b). Insights into the smooth-to-rough transitioning in Mycobacterium bolletii unravels a functional Tyr residue conserved in all mycobacterial MmpL family members. Mol. Microbiol. 99, 866-883. doi: 10.1111/mmi.13283

Brown-Elliott, B. A., Mann, L. B., Hail, D., Whitney, C., and Wallace, R. J. (2012). Antimicrobial susceptibility of nontuberculous mycobacteria from eye infections. Cornea 31, 900-906. doi: 10.1097/ICO.0b013e31823f8bb9

Brown-Elliott, B. A., and Philley, J. V. (2017). Rapidly growing mycobacteria. Microbiol. Spectr. 5, 1-19. doi: 10.1128/microbiolspec.TNMI7-0027-2016

Brown-Elliott, B. A., and Wallace, R. J. (2002). Clinical and taxonomic status of pathogenic nonpigmented or late-pigmenting rapidly growing mycobacteria. Clin. Microbiol. Rev. 15, 716-746. doi: 10.1128/CMR.15.4.716-746.2002

Cândido, P. H. C., Nunes, Lde S., Marques, E. A., Folescu, T. W., Coelho, F. S., de Moura, V. C. N., et al. (2014). Multidrug-resistant nontuberculous mycobacteria isolated from cystic fibrosis patients. J. Clin. Microbiol. 52, 2990-2997. doi: 10.1128/JCM.00549-14

Cambier, C. J., Takaki, K. K., Larson, R. P., Hernandez, R. E., Tobin, D. M., Urdahl, K. B., et al. (2014). Mycobacteria manipulate macrophage recruitment through coordinated use of membrane lipids. Nature 505, 218-222. doi: 10.1038/nature12799

Carvalho, R., de Sonneville, J., Stockhammer, O. W., Savage, N. D. L., Veneman, W. J., Ottenhoff, T. H. M., et al. (2011). A high-throughput screen for tuberculosis progression. PLOS ONE 6:e16779. doi: 10.1371/journal.pone. 0016779

Clay, H., Davis, J. M., Beery, D., Huttenlocher, A., Lyons, S. E., and Ramakrishnan, L. (2007). Dichotomous role of the macrophage in early Mycobacterium marinum infection of the zebrafish. Cell Host Microbe 2, 29-39. doi: 10.1016/j.chom.2007.06.004

Das, S., Garg, T., Chopra, S., and Dasgupta, A. (2019). Repurposing disulfiram to target infections caused by non-tuberculous mycobacteria. J. Antimicrob. Chemother. 74, 1317-1322. doi: 10.1093/jac/dkz018

Datta, D., Khatri, P., Singh, A., Saha, D. R., Verma, G., Raman, R., et al. (2018). Mycobacterium fortuitum-induced ER-mitochondrial calcium dynamics promotes calpain/caspase-12/caspase- 9 mediated apoptosis in fish macrophages. Cell Death Discov. 4:30. doi: 10.1038/s41420-018-0034-9

Davis, J. M., Clay, H., Lewis, J. L., Ghori, N., Herbomel, P., and Ramakrishnan, L. (2002). Real-time visualization of mycobacterium-macrophage interactions leading to initiation of granuloma formation in zebrafish embryos. Immunity 17, 693-702. doi: 10.1016/S1074-7613(02)00475-2

Davis, J. M., and Ramakrishnan, L. (2009). The role of the granuloma in expansion and dissemination of early tuberculous infection. Cell 136, 37-49. doi: 10.1016/j.cell.2008.11.014

Dubée, V., Bernut, A., Cortes, M., Lesne, T., Dorchene, D., Lefebvre, A.-L., et al. (2015). $\beta$-Lactamase inhibition by avibactam in Mycobacterium abscessus. J. Antimicrob. Chemother. 70, 1051-1058. doi: 10.1093/jac/dku510

Dumas, E., Christina Boritsch, E., Vandenbogaert, M., Rodríguez de la Vega, R. C., Thiberge, J.-M., Caro, V., et al. (2016). Mycobacterial pan-genome analysis suggests important role of plasmids in the radiation of type VII secretion systems. Genome Biol. Evol. 8, 387-402. doi: 10.1093/gbe/evw001

Dupont, C., Viljoen, A., Thomas, S., Roquet-Banères, F., Herrmann, J.-L., Pethe, K., et al. (2017). Bedaquiline inhibits the ATP synthase in Mycobacterium abscessus and is effective in infected zebrafish. Antimicrob. Agents Chemother. 61, e01225-17. doi: 10.1128/AAC.01225-17

Entwistle, F. M., and Coote, P. J. (2018). Evaluation of greater wax moth larvae, Galleria mellonella, as a novel in vivo model for non-tuberculosis mycobacteria infections and antibiotic treatments. J. Med. Microbiol. 67, 585-597. doi: $10.1099 /$ jmm.0.000696

García-Coca, M., Aguilera-Correa, J. J., Ibáñez-Apesteguía, A., Rodríguez-Sevilla, G., Romera-García, D., Mahíllo-Fernández, I., et al. (2019). Non-pigmented rapidly growing mycobacteria smooth and rough colony phenotypes pathogenicity evaluated using in vitro and experimental models. Pathog. Dis. 77:ftz051. doi: 10.1093/femspd/ftz051

Glickman, M. S., Cox, J. S., and Jacobs, W. R. (2000). A novel mycolic acid cyclopropane synthetase is required for cording, persistence, and virulence of Mycobacterium tuberculosis. Mol. Cell 5, 717-727. doi: $10.1016 /$ S1097-2765(00)80250-6
Gomes, M. C., and Mostowy, S. (2020). The case for modeling human infection in zebrafish. Trends Microbiol. 28, 10-18. doi: 10.1016/j.tim.2019.08.005

Houben, D., Demangel, C., van Ingen, J., Perez, J., Baldeón, L., Abdallah, A. M., et al. (2012). ESX-1-mediated translocation to the cytosol controls virulence of mycobacteria. Cell. Microbiol. 14, 1287-1298. doi: 10.1111/j.1462-5822.2012.01799.x

Johansen, M. D., Herrmann, J.-L., and Kremer, L. (2020). Non-tuberculous mycobacteria and the rise of Mycobacterium abscessus. Nat. Rev. Microbiol. 18, 392-407. doi: 10.1038/s41579-020-0331-1

Johansen, M. D., and Kremer, L. (2020). A zebrafish model of Mycobacterium kansasii infection reveals large extracellular cord formation. J. Infect. Dis. doi: 10.1093/infdis/jiaa187. [Epub ahead of print].

Laencina, L., Dubois, V., Le Moigne, V., Viljoen, A., Majlessi, L., Pritchard, J., et al. (2018). Identification of genes required for Mycobacterium abscessus growth in vivo with a prominent role of the ESX-4 locus. Proc. Natl. Acad. Sci. U.S.A. 115, E1002-E1011. doi: 10.1073/pnas.1713195115

Martiniano, S. L., Nick, J. A., and Daley, C. L. (2016). Nontuberculous mycobacterial infections in cystic fibrosis. Clin. Chest Med. 37, 83-96. doi: $10.1016 /$ j.ccm.2015.11.001

Oehlers, S. H., Cronan, M. R., Scott, N. R., Thomas, M. I., Okuda, K. S., Walton, E. M., et al. (2015). Interception of host angiogenic signalling limits mycobacterial growth. Nature 517, 612-615. doi: 10.1038/nature13967

Pagán, A. J., and Ramakrishnan, L. (2018). The formation and function of granulomas. Annu. Rev. Immunol. 36, 639-665. doi: 10.1146/annurev-immunol-032712-100022

Parti, R. P. S., Shrivastava, R., Srivastava, S., Subramanian, A. R., Roy, R., Srivastava, B. S., et al. (2008). A transposon insertion mutant of Mycobacterium fortuitum attenuated in virulence and persistence in a murine infection model that is complemented by Rv3291c of Mycobacterium tuberculosis. Microb. Pathog. 45, 370-376. doi: 10.1016/j.micpath.2008.08.008

Parti, R. P. S., Srivastava, S., Gachhui, R., Srivastava, K. K., and Srivastava, R. (2005). Murine infection model for Mycobacterium fortuitum. Microbes Infect. 7, 349-355. doi: 10.1016/j.micinf.2004.11.006

Prajsnar, T. K., Cunliffe, V. T., Foster, S. J., and Renshaw, S. A. (2008). A novel vertebrate model of Staphylococcus aureus infection reveals phagocyte-dependent resistance of zebrafish to non-host specialized pathogens. Cell. Microbiol. 10, 2312-2325. doi: 10.1111/j.1462-5822.2008. 01213.x

Prajsnar, T. K., Renshaw, S. A., Ogryzko, N. V., Foster, S. J., Serror, P., and Mesnage, S. (2013). Zebrafish as a novel vertebrate model to dissect enterococcal pathogenesis. Infect. Immun. 81, 4271-4279. doi: 10.1128/IAI.00976-13

Ratnatunga, C. N., Lutzky, V. P., Kupz, A., Doolan, D. L., Reid, D. W., Field, M., et al. (2020). The rise of non-tuberculosis mycobacterial lung disease. Front. Immunol. 11:303. doi: 10.3389/fimmu.2020.00303

Raynaud, C., Daher, W., Johansen, M., Roquet-Baneres, F., Blaise, M., Onajole, O. K., et al. (2019). Active benzimidazole derivatives targeting the MmpL3 transporter in Mycobacterium abscessus. ACS Infect. Dis. 6, 324-337. doi: 10.1021/acsinfecdis.9b00389

Richards, C. J., and Olivier, K. N. (2019). Nontuberculous mycobacteria in cystic fibrosis. Semin. Respir. Crit. Care Med. 40, 737-750. doi: 10.1055/s-0039-1693706

Roux, A.-L., Catherinot, E., Ripoll, F., Soismier, N., Macheras, E., Ravilly, S., et al. (2009). Multicenter study of prevalence of nontuberculous mycobacteria in patients with cystic fibrosis in france. J. Clin. Microbiol. 47, 4124-4128. doi: 10.1128/JCM.01257-09

Roux, A.-L., Viljoen, A., Bah, A., Simeone, R., Bernut, A., Laencina, L., et al. (2016). The distinct fate of smooth and rough Mycobacterium abscessus variants inside macrophages. Open Biol. 6:160185. doi: 10.1098/rsob.160185

Saito, H., and Tasaka, H. (1969). Comparison of the pathogenicity for mice of Mycobacterium fortuitum and Mycobacterium abscessus. J. Bacteriol. 99, 851-855. doi: 10.1128/JB.99.3.851-855.1969

Sermet-Gaudelus, I., Le Bourgeois, M., Pierre-Audigier, C., Offredo, C., Guillemot, D., Halley, S., et al. (2003). Mycobacterium abscessus and children with cystic fibrosis. Emerging Infect. Dis. 9, 1587-1591. doi: 10.3201/eid0912. 020774

Skolnik, K., Kirkpatrick, G., and Quon, B. S. (2016). Nontuberculous mycobacteria in cystic fibrosis. Curr. Treat Options Infect. Dis. 8, 259-274. doi: $10.1007 /$ s40506-016-0092-6 
Staropoli, J. F., and Branda, J. A. (2008). Cord formation in a clinical isolate of Mycobacterium marinum. J. Clin. Microbiol. 46, 2814-2816. doi: 10.1128/JCM.00197-08

Takaki, K., Cosma, C. L., Troll, M. A., and Ramakrishnan, L. (2012). An in vivo platform for rapid high-throughput antitubercular drug discovery. Cell Rep. 2, 175-184. doi: 10.1016/j.celrep.2012.06.008

Talaat, A. M., Trucksis, M., Kane, A. S., and Reimschuessel, R. (1999). Pathogenicity of Mycobacterium fortuitum and Mycobacterium smegmatis to goldfish, Carassius auratus. Vet. Microbiol. 66, 151-164. doi: 10.1016/S0378-1135(99)00002-4

Tobin, D. M., Vary, J. C., Ray, J. P., Walsh, G. S., Dunstan, S. J., Bang, N. D., et al. (2010). The lta4h locus modulates susceptibility to mycobacterial infection in zebrafish and humans. Cell 140, 717-730. doi: 10.1016/j.cell.2010.02.013

Torraca, V., and Mostowy, S. (2018). Zebrafish infection: from pathogenesis to cell biology. Trends Cell Biol. 28, 143-156. doi: 10.1016/j.tcb.2017.10.002

Tortoli, E., Fedrizzi, T., Meehan, C. J., Trovato, A., Grottola, A., Giacobazzi, E., et al. (2017). The new phylogeny of the genus Mycobacterium: the old and the news. Infect. Genet. Evol. 56, 19-25. doi: 10.1016/j.meegid.2017.10.013

van der Sar, A. M., Appelmelk, B. J., Vandenbroucke-Grauls, C. M. J. E., and Bitter, W. (2004). A star with stripes: zebrafish as an infection model. Trends Microbiol. 12, 451-457. doi: 10.1016/j.tim.2004.08.001

Vergunst, A. C., Meijer, A. H., Renshaw, S. A., and O'Callaghan, D. (2010). Burkholderia cenocepacia creates an intramacrophage replication niche in zebrafish embryos, followed by bacterial dissemination and establishment of systemic infection. Infect. Immun. 78, 1495-1508. doi: 10.1128/IAI. 00743-09
Vilchèze, C., Molle, V., Carrère-Kremer, S., Leiba, J., Mourey, L., Shenai, S., et al. (2014). Phosphorylation of KasB regulates virulence and acid-fastness in Mycobacterium tuberculosis. PLoS Pathog. 10:e1004115. doi: 10.1371/journal.ppat.1004115

Viljoen, A., Gutiérrez, Ana, V.ictoria, Dupont, C., Ghigo, E., and Kremer, L. (2018). A simple and rapid gene disruption strategy in Mycobacterium abscessus: on the design and application of glycopeptidolipid mutants. Front. Cell. Infect. Microbial. 8:69. doi: 10.3389/fcimb.2018.00069

Volkman, H. E., Clay, H., Beery, D., Chang, J. C. W., Sherman, D. R., and Ramakrishnan, L. (2004). Tuberculous granuloma formation is enhanced by a mycobacterium virulence determinant. PLoS Biol. 2:e367. doi: 10.1371/journal.pbio.0020367

Wallace, R. J., Swenson, J. M., Silcox, V. A., Good, R. C., Tschen, J. A., and Stone, M. S. (1983). Spectrum of disease due to rapidly growing mycobacteria. Rev. Infect. Dis. 5, 657-679. doi: 10.1093/clinids/5.4.657

Conflict of Interest: The authors declare that the research was conducted in the absence of any commercial or financial relationships that could be construed as a potential conflict of interest.

Copyright (c) 2020 Johansen and Kremer. This is an open-access article distributed under the terms of the Creative Commons Attribution License (CC BY). The use, distribution or reproduction in other forums is permitted, provided the original author(s) and the copyright owner(s) are credited and that the original publication in this journal is cited, in accordance with accepted academic practice. No use, distribution or reproduction is permitted which does not comply with these terms. 


\section{OPEN ACCESS}

Edited by:

Gheyath Khaled Nasrallah,

Qatar University, Qatar

Reviewed by:

Michael Povelones,

University of Pennsylvania,

United States

Mohammad Farhan,

Hamad bin Khalifa University, Qatar

${ }^{*}$ Correspondence:

Kristin Michel

kmichel@ksu.edu

${ }^{\dagger}$ Present address:

Xin Zhang,

Department of Pharmacodynamics, University of Florida, Gainesville,

FL, United States

Specialty section:

This article was submitted to

Parasite and Host

a section of the journal

Frontiers in Cellular and

Infection Microbiology

Received: 22 July 2020 Accepted: 30 November 2020 Published: 15 January 2021

Citation:

Zhang X, Li M, El Moussawi L, Saab S,

Zhang S, Osta MA and Michel $K$ (2021) CLIPB10 is a Terminal Protease

in the Regulatory Network That

Controls Melanization in the African Malaria Mosquito Anopheles gambiae.

Front. Cell. Infect. Microbiol. 10:585986.

doi: 10.3389/fcimb.2020.585986

\section{CLIPB10 is a Terminal Protease in the Regulatory Network That Controls Melanization in the African Malaria Mosquito Anopheles gambiae}

\author{
Xin Zhang ${ }^{1 \dagger}$, Miao Li $^{1}$, Layla El Moussawi ${ }^{2}$, Sally Saab ${ }^{2}$, Shasha Zhang ${ }^{1,3}$, Mike A. Osta ${ }^{2}$ \\ and Kristin Michel ${ }^{1 *}$ \\ ${ }^{1}$ Division of Biology, Kansas State University, Manhattan, KS, United States, ${ }^{2}$ Department of Biology, American University of \\ Beirut, Beirut, Lebanon, ${ }^{3}$ Department of Entomology, China Agricultural University, Beijing, China
}

Humoral immune responses in animals are often tightly controlled by regulated proteolysis. This proteolysis is exerted by extracellular protease cascades, whose activation culminates in the proteolytic cleavage of key immune proteins and enzymes. A model for such immune system regulation is the melanization reaction in insects, where the activation of prophenoxidase (proPO) leads to the rapid formation of eumelanin on the surface of foreign entities such as parasites, bacteria and fungi. ProPO activation is tightly regulated by a network of so-called clip domain serine proteases, their proteolytically inactive homologs, and their serpin inhibitors. In Anopheles gambiae, the major malaria vector in sub-Saharan Africa, manipulation of this protease network affects resistance to a wide range of microorganisms, as well as host survival. However, thus far, our understanding of the molecular make-up and regulation of the protease network in mosquitoes is limited. Here, we report the function of the clip domain serine protease CLIPB10 in this network, using a combination of genetic and biochemical assays. CLIPB10 knockdown partially reversed melanotic tumor formation induced by Serpin 2 silencing in the absence of infection. CLIPB10 was also partially required for the melanization of ookinete stages of the rodent malaria parasite Plasmodium berghei in a refractory mosquito genetic background. Recombinant serpin 2 protein, a key inhibitor of the proPO activation cascade in An. gambiae, formed a SDS-stable protein complex with activated recombinant CLIPB10, and efficiently inhibited CLIPB10 activity in vitro at a stoichiometry of 1.89:1. Recombinant activated CLIPB10 increased PO activity in Manduca sexta hemolymph ex vivo, and directly activated purified $M$. sexta proPO in vitro. Taken together, these data identify CLIPB10 as the second protease with prophenoloxidase-activating function in An. gambiae, in addition to the previously described CLIPB9, suggesting functional redundancy in the protease network that 
controls melanization. In addition, our data suggest that tissue melanization and humoral melanization of parasites are at least partially mediated by the same proteases.

Keywords: innate immunity, melanization, serine protease, serpin (serine proteinase inhibitor), phenoloxidase

\section{INTRODUCTION}

Melanization, the biochemical formation and deposition of melanin fulfills diverse biological functions in living organisms (Cerenius and Soderhall, 2004; Vavricka et al., 2010; Sugumaran and Barek, 2016; Pavan et al., 2020). In arthropods, including mosquitoes the production of eumelanin is a broad-spectrum immune response against viruses (Rodriguez-Andres et al., 2012), bacteria (Hillyer et al., 2003; Yassine et al., 2014), fungi (Yassine et al., 2012a), chromista (Brey et al., 1988), and other eukaryotic parasites (Christensen, 1981; Michel et al., 2005; Habtewold et al., 2008). In mosquitoes, melanization initially received much attention as a selectable phenotype that confers refractoriness to parasites of public health importance including, malaria parasites and filarial worms (Collins et al., 1986; Chen and Laurence, 1987; Hurd et al., 2005). Subsequent genetic studies identified single genes whose knockdown $(\mathrm{kd})$ triggers a potent melanotic response against Plasmodium ookinetes (Osta et al., 2004; Michel et al., 2005; Frolet et al., 2006; Nakhleh et al., 2017a), drawing considerable attention to the potential application of this response in controlling vector competence. Indeed the non-vector mosquito, Anopheles quadriannulatus, was shown to trigger a potent immune response to Plasmodium ookinetes characterized by a significant melanization of $P$. berghei ookinetes and occasionally of $P$. falciparum (Habtewold et al., 2008).

The infection-induced melanization in An. gambiae is tightly regulated by the complement-like pathway, specifically the thioester-containing protein 1 (TEP1), which upon activation is deposited on the surface of entities that are recognized as damaged or foreign (Blandin et al., 2004; Yassine et al., 2012a; Povelones et al., 2013). TEP1 binding to microbial surfaces triggers, in a yet unknown mechanism, the activation of a complex downstream network of clip domain serine proteases (CLIPs) constituted of both catalytic proteases (cSPs) and their non-catalytic homologs ( $\mathrm{cSPHs}$ ). A terminal protease in the cascade ultimately cleaves and activates the zymogen prophenoloxidase (proPO) into active phenoloxidase (PO), the rate-limiting enzyme in melanogenesis (Nakhleh et al., 2017b; Rhodes and Michel, 2017; Bartholomay and Michel, 2018). Melanin formation on surfaces of microbes is thought to hinder their intake of nutrients, while toxic intermediates, such as reactive oxygen and reactive nitrogen species, may also cause cellular damage (Nappi et al., 2009).

There are 110 cSPs and cSPHs currently annotated in the $A n$. gambiae genome, which are divided into five sub-groups called CLIPA-E, based on phylogeny, clip-domain structure, and domain arrangement (Christophides et al., 2002; An et al., 2011; Cao et al., 2017). Groups B-D largely contain cSPs, with one or more clip domains at the amino terminus and a chymotrypsin-like protease domain at the carboxyl terminus. In contrast, all members of the CLIPA family are cSPHs and contain one to five clip domains at the $\mathrm{N}$-terminus and a protease homolog domain at the carboxyl terminus, while CLIPEs include several members containing one catalytic domain plus one or more protease homolog domains in the same molecule. CLIPBs are core components of CLIP cascades that are secreted into the hemolymph as zymogens and are activated sequentially by specific cleavage at the linker region between the clip and protease domains by an upstream protease (An et al., 2011; Zhang et al., 2016). Studies in other model insects have shown that CLIPCs act upstream of CLIPBs in these cascades and that the terminal conversion of proPO to active $\mathrm{PO}$ is mediated by prophenoloxidase activating proteins (PAPs), which in all insects examined so far, always belong to the CLIPB family [reviewed in (Kanost and Jiang, 2015)].

ProPO activation cascades have to be strictly regulated to avoid excessive production of harmful byproducts, which could cause systematic damage. Serpins are a superfamily of serine protease inhibitors, which form covalent inhibitory complexes with target proteases (Gettins, 2002; Law et al., 2006; Whisstock and Bottomley, 2006; Meekins et al., 2016). Suppression of proPO activation in insects is mainly achieved through the inhibition of cSPs by a highly conserved serpin, called serpin-3 in M. sexta (Zhu et al., 2003), spn27A in Drosophila melanogaster (De Gregorio et al., 2002; Ligoxygakis, 2002; Tang et al., 2006), and SRPN2 in An. gambiae (Michel et al., 2005; An et al., 2011).

Two principal models of melanization observed in mosquitoes, tissue melanization and humoral melanization of microbes have been used in An. gambiae to identify putative CLIPA and B family members of the proPO activation cascade. Tissue melanization, induced by the depletion of SRPN2, the principal serpin that regulates proPO activation in An. gambiae, results in the formation of melanotic pseudo-tumors in the hemocoel in the absence of foreign objects or microbial infection (Michel et al., 2005; Michel et al., 2006; An et al., 2011). Humoral melanization, characterized by the deposition of melanin on foreign surfaces, can be induced through implantation of Sephadex beads or the injection of bacteria and fungal spores into the thorax of adult An. gambiae (Chun et al., 1995; Schnitger et al., 2007; Yassine et al., 2012b). In addition, humoral melanization of ookinete stages of rodent malaria parasites is induced through knockdown of the c-type lectins CTL4 and CTLMA2 in An. gambiae strains that are otherwise susceptible to these parasites (Osta et al., 2004). Targeted RNAi screens in these two models of melanization coupled with biochemical studies revealed a complex CLIP network in An. gambiae, which not only entails proteolytic activation among CLIPBs (An et al., 2011; Zhang et al., 2016), but also hierarchical interactions between CLIPAs (Yassine et al., 
2014; Nakhleh et al., 2017a; El Moussawi et al., 2019). In addition, the list of CLIPs involved in humoral melanization is dependent on the genetic background as well as the target of melanization (Paskewitz et al., 2006; Volz et al., 2006). Studies in the yellow fever mosquito Aedes aegypti have also suggested that humoral and tissue melanization are regulated by distinct proPO activation pathways (Zou et al., 2010). Further complexity of the system can arise through the existence of several PAPs that may act in parallel, as suggested through biochemical analysis of the proPO activation cascades in the model lepidopteran Manduca sexta (Jiang et al., 1998; Jiang et al., 2003a; Jiang et al., 2003b; Zhu et al., 2003). Whether parallel proPO activation cascades exist and are invoked separately in tissue and humoral melanization in An. gambiae is currently unknown.

To address these open questions, this study focused on the analysis of An. gambiae CLIPB10 (Vectorbase Id in AGAMP4.4: AGAP029770, NCBI protein ID: XM_312744.4). CLIPB10 is the closest paralog to CLIPB9, the only bona fide PAP known in mosquitoes (An et al., 2011). CLIPB9 and B10, together with CLIPB8 are physically clustered in a $10 \mathrm{~Kb}$ region on chromosome $2 \mathrm{R}$, and are the products of two consecutive duplications of their ancestral gene (Waterhouse et al., 2007; An et al., 2011). In addition, CLIPB10 was shown previously to contribute to humoral melanization of Sephadex beads (Paskewitz et al., 2006). Here, we investigate whether CLIPB10 also contributes to the humoral melanization of rodent malaria parasites as well as tissue melanization. In addition, we explore its enzymatic interactions with its closest CLIPB paralogs and the regulatory effector SRPN2, thus pinpointing CLIPB10 location in the proPO activation cascade.

\section{MATERIALS AND METHODS}

\section{Mosquito Strain and Maintenance}

The An. gambiae G3 strain (MRA-112) was obtained through the MR4 Anopheles Program at the CDC in 2007, and has since then be maintained in the Michel laboratory as described previously (An et al., 2011). Heparinized horse blood (Plasvacc, Templeton, CA, USA) was provided through an artificial membrane feeding system.

\section{RNAi Experiments Performed in Adult Female Mosquitoes}

DsRNA were synthesized as described previously using primers listed in Table S1 (Michel et al., 2005). One to three day old female were injected with $138 \mathrm{nl}$ of $1.5 \mu \mathrm{g} / \mu \mathrm{l}$ for each dsRNA. For single knockdown (kd) and double kd controls, dsGFP was added to keep the total dsRNA dose constant at $207 \mathrm{ng} /$ mosquito between treatment and controls, as described previously (Zhang et al., 2016).

\section{Ethics Statement and Plasmodium berghei Parasite Infections}

This study was carried out in accordance with the recommendations in the Guide for the Care and Use of Laboratory Animals of the National Institutes of Health (Bethesda, USA). Animal protocol was approved by the
Institutional Animal Care and Use committee IACUC of the American University of Beirut (permit number 17-10-451). The IACUC functions in compliance with the Public Health Service Policy on the Humane Care and Use of Laboratory Animals (USA), and adopts the Guide for the Care and Use of Laboratory Animals of the National Institutes of Health. P. berghei (strain PbGFPCON) constitutively expressing GFP was propagated in BALB/c mice (Franke-Fayard et al., 2004). Mosquitoes were allowed to feed on 5- to 6-week-old anesthetized mice containing a blood parasitemia of $4 \%-6 \%$ for $20 \mathrm{~min}$ at $20^{\circ} \mathrm{C}$. Mosquitoes were then maintained on $10 \%$ sucrose solution at $20^{\circ} \mathrm{C}$ with a 12 -h day-night cycle. Dissection and fixation of mosquito midguts was performed 7 days post blood feeding, followed by counting of fluorescent oocysts and melanized ookinetes with Zeiss fluorescence microscope as previously described (Volz et al., 2006). Five independent biological replicates with at least 42 mosquitoes each were examined.

\section{Melanotic Tumor Phenotype Assessment in Adult Mosquitoes}

DsRNA-injected mosquitoes were generated as described in 2.2 above, and maintained on sugar water for 21 days. To quantify melanotic pseudotumors, abdominal wall of each mosquito was dissected and examined under 40× magnification with Axio Imager A1 microscope (Zeiss) equipped with AxioCamMR5 (Zeiss). Image $\mathrm{J}$ was used to quantify the melanized area per abdomen. Two independent biological replicates with 40 mosquitoes each were used.

\section{Reverse Transcription Quantitative PCR}

Efficiency of gene $\mathrm{kd}$ was measured by reverse transcription quantitative PCR (RT-qPCR). Briefly, total RNA was isolated from mosquitoes at day 4 post dsRNA injection using TRIzol reagent (Invitrogen). Total RNA (100 ng) was used as the template to synthesize cDNA using iScript cDNA synthesis kit (Bio-Rad) according to the manufacturer's instructions. RTqPCR was set up by mixing $0.5 \mu \mathrm{l}$ cDNA, $0.8 \mu \mathrm{M}$ primers, and iQ SYBR Green Supermix, followed by amplification on ABI StepOnePlus system (Applied Biosystems). Relative expression of genes of interest was calculated by $\Delta \Delta \mathrm{Ct}$ method using AgRPS7 as internal reference gene. Three technical replicates were measured for each sample and primer pair.

\section{Recombinant Serine Protease Expression and Purification}

The coding region of proCLIPB10 was amplified by PCR from An. gambiae adult cDNA using primers listed in Table S1. The forward primer contains a NotI restriction site, and the reverse primer includes codons for six histidines followed by a stop codon and a HindIII restriction site. The PCR product was digested with NotI and HindIII and cloned into the same sites of the expression vector pFastBacI (Invitrogen). The resulting expression vector was used as the template to produce the mutant proCLIPB $10_{\mathrm{Xa}}$ expression plasmid following the instructions of QuikChange Multi SiteDirected Mutagenesis Kit (Agilent). CLIPB10 activation site LADR was replaced by IEGR to allow the cleavage and activation 
by Factor Xa (New England Biolabs). Recombinant constructs were transfected to Sf9 cells using Bac-to-Bac system (Invitrogen), followed by generation of recombinant baculovirus. To express proCLIPB10 ${ }_{\mathrm{Xa}}, 800 \mathrm{ml}$ of $\mathrm{Sf} 9$ cells $\left(2 \times 10^{6}\right.$ cells $\left./ \mathrm{ml}\right)$ were infected with the recombinant baculovirus at a multiplicity of infection of 1 and cultured at $27^{\circ} \mathrm{C}$ with shaking at $140 \mathrm{rpm}$ for 4 days. The medium containing secreted proCLIPB10 ${ }_{\mathrm{Xa}}$ was harvested by centrifugation at $4^{\circ} \mathrm{C}, 500 \times \mathrm{g}$ for $20 \mathrm{~min}$. $\mathrm{Na}_{2} \mathrm{HPO}_{4}$ was added to the $800 \mathrm{ml}$ of cell-free medium to a final concentration of $10 \mathrm{mM}$, followed by dialysis using $40 \mathrm{~mm}$-wide regenerated cellulose dialysis tubing (Fisher Scientific) against $4 \mathrm{~L}$ of $20 \mathrm{mM} \mathrm{Na}_{2} \mathrm{HPO}_{4}, \mathrm{pH} 8.0$ thrice for $12 \mathrm{~h}$ each at $4^{\circ} \mathrm{C}$. The dialyzed medium was supplemented with $50 \mathrm{mM} \mathrm{Na}_{2} \mathrm{HPO}_{4}, 300 \mathrm{mM} \mathrm{NaCl}$, and $10 \mathrm{mM}$ imidazole, followed by Ni-NTA chromatography (Qiagen) according to manufacturer's instruction. All $10 \mathrm{ml}$ elution fractions that contained proCLIPB10 ${ }_{\mathrm{Xa}}$ were pooled and dialyzed using $40 \mathrm{~mm}$ wide regenerated cellulose dialysis tubing against $2 \mathrm{~L}$ of $20 \mathrm{mM}$ Tris, $20 \mathrm{mM} \mathrm{NaCl}, \mathrm{pH} 8.0$ twice at $4^{\circ} \mathrm{C}$. Further purification was performed with Q Sepharose column according to manufacturer's protocol (GE Healthcare). Fractions containing proCLIPB10 ${ }_{\mathrm{Xa}}$ were stored at $-80^{\circ} \mathrm{C}$ for future use.

Recombinant proCLIPB8, proCLIPB9, proCLIPB8 $\mathrm{Xa}$, proCLIPB9 $_{\mathrm{Xa}}$ and SRPN2 were expressed and purified as described previously (An et al., 2011; Zhang et al., 2016).

\section{Activation of Recombinant Zymogens}

To activate recombinant CLIPB9 ${ }_{\mathrm{Xa}}$ and CLIPB10 $\mathrm{Xa}, 2.5 \mu \mathrm{g}$ of each purified zymogen was incubated with $1 \mu \mathrm{g}$ of bovine Factor $\mathrm{Xa}$ (New England Biolabs) in a total volume of $50 \mu \mathrm{l}$ in reaction buffer (20 mM Tris, $100 \mathrm{mM} \mathrm{NaCl}, 2 \mathrm{mM} \mathrm{CaCl}_{2}, \mathrm{pH} \mathrm{8.0)}$ at $37^{\circ} \mathrm{C}$ overnight. Two negative controls were set up in parallel, in which either Factor Xa or the zymogen was replaced with same volume of buffer. Cleavage of the zymogen was examined by loading $8 \mu \mathrm{l}$ of the activation reaction to $10 \%$ SDS-PAGE followed by Coomassie blue staining for visualization.

\section{Substrate Screening of Active CLIPB10}

To identify a suitable commercial substrate to measure CLIPB10 Xa amidase activity, the following chromogenic peptides were tested: N-benzoyl-Ile-Glu-Ala-Arg- $p$-nitroanilide (IEARpNa), N-benzoylPhe-Val-Arg- $p$-nitroanilide (FVR $p \mathrm{Na}$ ), N-benzoyl-Pro-Phe-Arg- $p$ nitroanilide (PFR $p$ Na), N-benzoyl-Asn-Asn-Asp-Arg- $p$ nitroanilide (NNDR $p$ Na), N-benzoyl-Ile-Glu-Gly-Arg$p$-nitroanilide (IEGR $p \mathrm{Na}$ ), N-benzoyl-Ala-Ala-Pro-Phe- $p$ nitroanilide (AAPF $p \mathrm{Na}$ ), N-benzoyl-Ile-Ala-Gln-Arg$p$-nitroanilide (IAQR $p \mathrm{Na}$ ), N-benzoyl-Val-Gly-Asn-Lys- $p$ nitroanilide (VGNKpNa). CLIPB10 Xa was activated as described in Reverse Transcription Quantitative PCR, and $3 \mu \mathrm{l}$ of the activation reaction was added to $200 \mu \mathrm{l}$ of assay buffer $(0.1 \mathrm{M}$ Tris, $0.1 \mathrm{M} \mathrm{NaCl}$, $5 \mathrm{mM} \mathrm{CaCl}_{2}, \mathrm{pH}$ 8.0) containing $500 \mu \mathrm{M}$ synthetic substrate. Amidase activity was measured by the change of absorbance at $405 \mathrm{~nm}$ over $20 \mathrm{~min}$ at room temperature. One unit was defined as $\Delta \mathrm{A}_{405}=0.001 / \mathrm{min}$. A baseline control was set up by measuring the amidase activity of Factor Xa in the absence of proCLIPB $10_{\mathrm{Xa}}$ with different substrates. Activity of CLIPB10 ${ }_{X a}$ was calculated by subtracting Factor Xa activity from activated CLIPB10 ${ }_{\mathrm{Xa}}$ in the presence of Factor Xa. All measurements were performed in two independent replicates.

\section{Protease-Serpin Complex Formation and MALDI-TOF MS Analysis}

Recombinant proCLIPB10 ${ }_{\mathrm{Xa}}$ was activated by Factor $\mathrm{Xa}$ as described above in 2.5 , and $20 \mu \mathrm{l}$ of the activation reaction was incubated with $2 \mu \mathrm{l}$ of $5 \mu \mathrm{g} / \mu \mathrm{l}$ purified recombinant SRPN2 (rSRPN2) at room temperature for $1.5 \mathrm{~h}$. Formation of the protease-serpin complex was visualized by $10 \%$ SDS-PAGE stained with Coomassie blue. Two bands at $\sim 72$ and $\sim 55 \mathrm{kDa}$ were excised from the gel and subjected to in-gel trypsin digestion and Electrospray ionization mass spectrometry analysis (Bruker Daltonics HCT Ultra) at the Biotechnology/Proteomics Core Facility, Kansas State University. Mass spectra were analyzed using Scaffold (4.10.0).

\section{Inhibition of CLIPB10 xa by rSRPN2}

To explore the stoichiometry of SRPN2 inhibition of CLIPB10, purified rSRPN2 was incubated with $0.1 \mu \mathrm{g}$ of in vitro activated CLIPB10 $_{\mathrm{Xa}}$ at molar ratios of $0,0.125,0.25,0.75$, and 1.25 , respectively, in the presence of $1 \mu \mathrm{lBSA}(2 \mu \mathrm{g} / \mu \mathrm{l})$ at room temperature for $20 \mathrm{~min}$ in $20 \mathrm{mM}$ Tris, $100 \mathrm{mM} \mathrm{NaCl}, \mathrm{pH} 8.0$. Amidase activity was determined in the presence of $500 \mu \mathrm{M}$ IEAR $p \mathrm{Na}$ as described in 2.5 above. Amidase activity at 0:1 (rSRPN2: CLIPB10 ${ }_{\mathrm{Xa}}$ ) was defined as $100 \%$. All assays were performed in triplicate.

\section{Assessment of CLIPB8 and B9 Cleavage by Recombinant CLIPB10}

CLIPB10 $\mathrm{Xa}$ was activated by incubating $2.5 \mu \mathrm{g}$ of purified zymogen with $2.0 \mu \mathrm{g}$ of bovine Factor Xa (New England Biolabs) in a total volume of $50 \mu \mathrm{l}$ in reaction buffer $(20 \mathrm{mM}$ Tris, $100 \mathrm{mM} \mathrm{NaCl}, 2 \mathrm{mM} \mathrm{CaCl}_{2}, \mathrm{pH} 8.0$ ) at $37^{\circ} \mathrm{C}$ overnight. Two negative controls were set up in parallel, in which either Factor Xa or the zymogen was replaced with same volume of buffer. Two microliters of the activation reaction was then incubated with $0.8 \mathrm{ul}$ of $126 \mathrm{ng} / \mathrm{ul}$ of recombinant purified proCLIPB8 and $0.6 \mathrm{ul}$ of $168 \mathrm{ng} / \mathrm{ul}$ of recombinant purified proCLIPB9 in $20 \mathrm{mM}$ Tris, $150 \mathrm{mM} \mathrm{NaCl}, \mathrm{pH} 8.0$, respectively, at room temperature for $10 \mathrm{~min}$. Potential cleavage of the CLIPB8 and CLIPB9 zymogens by activated CLIPB10 ${ }_{\mathrm{Xa}}$, respectively was examined by Western blot. The reactions were treated with $6 \times$ SDS loading buffer (supplemented with $\beta$ mercaptoethanol), and heated at $95^{\circ} \mathrm{C}$ for $5 \mathrm{~min}$. Proteins were separated on $12 \%$ SDS-PAGE, and transferred onto a nitrocellulose membrane. Membranes were blocked with 5\% milk, and incubated with mouse anti-His antibody $(1: 2,000)$ as primary antibody and 1:2,000 diluted goat anti-mouse IgG APconjugated secondary antibody (Promega). Alternatively, membranes were incubated with rabbit anti-CLIPB8 antibody [(Zhang et al., 2016), 1:500] and rabbit anti-CLIPB9 antibody [(An et al., 2011), 1:500] as primary antibody, respectively, and 1:5,000 diluted goat anti-rabbit IgG AP-conjugated secondary antibody (Promega). All Western blots were visualized by AP conjugate substrate kit (Bio-Rad). 


\section{Activation of proPO in Plasma}

CLIPB9 $_{\mathrm{Xa}}$ or CLIPB10 $\mathrm{Xa}$ were activated by Factor Xa as described above in Reverse Transcription Quantitative PCR. $8 \mu$ l of the activation mixture was incubated with $2 \mu \mathrm{l}$ of 1:10 diluted plasma collected from day-2 fifth instar M. sexta larvae at $37^{\circ} \mathrm{C}$ for $30 \mathrm{~min}$. Samples were treated with $6 \times$ SDS loading buffer (supplemented with $\beta$-mercaptoethanol), heated at $95^{\circ} \mathrm{C}$ for $5 \mathrm{~min}$. Proteins were separated on $10 \%$ SDS-PAGE, and transferred onto a PVDF membrane. The membrane was blocked with $5 \%$ milk, and incubated with rabbit anti- $M$. sexta PPO $(1: 2,000)$ as primary antibody and 1:20,000 diluted goat anti-rabbit IgG AP-conjugated secondary antibody (Promega). The Western blot was visualized by AP conjugate substrate kit (Bio-Rad). An $8-\mu$ l volume of the activation mixture was also used to measure PO activity by adding dopamine to a $2 \mathrm{mM}$ final concentration in $50 \mathrm{mM}$ sodium phosphate, $\mathrm{pH}$ 6.5. One unit of $\mathrm{PO}$ activity was defined as $\Delta \mathrm{A}_{470}=0.001 / \mathrm{min}$. Three technical replicates were performed.

\section{Cleavage of Purified proPO In Vitro}

CLIPB9 ${ }_{\mathrm{Xa}}$ or CLIPB10 ${ }_{\mathrm{Xa}}$ were activated by Factor Xa as described above in Reverse Transcription Quantitative PCR, and $5 \mu \mathrm{l}$ of activation mixture was incubated with $0.6 \mu \mathrm{l}$ of $100 \mathrm{ng} / \mu \mathrm{l}$ purified $M$. sexta proPO (kindly provided by Maureen Gorman, Kansas State University) at $37^{\circ} \mathrm{C}$ for $30 \mathrm{~min}$. Samples were then subjected to immunoblotting as described above in 2.10 .

\section{Statistical Analyses}

Statistical analyses were executed using GraphPad Prism 6.07 Software (GrapPad Software Inc.). Melanotic tumor formation data were evaluated for normality of distribution using ShapiroWilk normality test; data were analyzed using (i) Mann Whitney Utest, if comparing two treatment groups, or (ii) Kruskal Wallis test for multiple treatment groups, with Dunn's Multiple Comparison post-test $(P<0.05)$. Parasite infection data were analyzed by evaluating potential differences in prevalence and infection intensity separately for (i) live oocyst numbers and (ii) melanized ookinetes. Potential differences in prevalence were analyzed using the $\chi^{2}$ test, by comparing each treatment group to the CTL4/B10 treatment, followed by Bonferroni correction $(P<0.05)$. Live oocyst and melanized ookinete infection intensity data, respectively were evaluated for normality of distribution using Shapiro-Wilk normality test. Potential differences in infection intensity were analyzed using Kruskal Wallis test for multiple treatment groups, with Dunn's Multiple Comparison post-test $(P<0.05)$. All enzymatic activity data were evaluated using One-Way ANOVA, with Newman-Keuls post-test $(P<0.05)$.

\section{RESULTS}

\section{CLIPB10 Is Required for Tissue and Plasmodium Melanization}

To test the involvement of CLIPB10 in An. gambiae tissue melanization, we synthesized dsRNA to perform single and double $\mathrm{kd}$ of SRPN2 and CLIPB10. The corresponding dsRNA was injected into 1-2 day female adults, and RT-qPCR was used to test the knockdown efficiency 4 days post injection (Figure S1). Transcription level of CLIPB10 was reduced by over $95 \%$ after dsCLIPB10 or $d s S R P N 2 / d s C L I P B 10$ injection. SRPN2 expression level was reduced by $50 \%$, which is similar to previously reported values (Michel et al., 2005; An et al., 2011; Zhang et al., 2016).

The kd of SRPN2 caused severe melanotic tumors on the abdomen of mosquitoes, which were partially reversed by CLIPB10 kd in SRPN2-depleted mosquitoes (Figure 1A). Quantification of melanotic areas per abdomen demonstrated a significant reduction in melanization in dsSRPN2/dsCLIPB10 as compared to dsSRPN2-treated mosquitoes (Figure 1B). Neither dsGFP nor dsCLIPB10 injection alone caused recognizable melanotic tumors in mosquitoes.

To test whether CLIPB10 is also required for melanization of malaria parasites, adult female mosquitoes were injected with dsCTL4 and dsCLIPB10 to induce gene silencing, and subsequently given an infectious blood meal containing GFPexpressing P. berghei. Live oocysts with green fluorescence and dead melanized ookinetes were scored in the midguts of dissected mosquitoes at day 7 after blood feeding (Table S2). Silencing of CLIPB10 did not alter parasite development, as the median numbers of live oocysts and melanized ookinetes per gut in dsCLIPB10 mosquitoes was similar to the numbers observed in the dsLacZ-injected control group (Figure 1C and Table S3). However, in dsCTL4 mosquitoes, silencing CLIPB10 tripled the median number of live oocysts, and quartered the median number of melanized ookinetes (Figure 1C and Table S3). To further investigate the impact of CLIPB10 $\mathrm{kd}$ on parasite development, we determined the prevalence of parasite infection by determining the percentage of mosquitoes whose guts carried live oocysts. In addition, we also analyzed the percentage of mosquitoes whose midguts contained melanized ookinetes (Table S4 and Figure 1C). Co-silencing CLIPB10 and CTL4 did not change the prevalence of live oocysts as compared to CTL4-silenced mosquitoes ( $\chi^{2}$ test, $P=0.2905$, Table S4), suggesting that CLIPB10 is probably not involved in parasite killing. In contrast, co-silencing CLIPB10 and CTL4 significantly reduced the percentage of mosquitoes bearing melanized ookinetes ( $\chi^{2}$ test, $P=0.0003$, Table S4), further supporting the role of CLIPB10 in the humoral melanization of parasites.

\section{Active Recombinant proCLIPB10 Xa Exhibits Amidase Activity}

The reverse genetic analysis revealed that CLIPB10 is required for humoral and tissue melanization. To determine whether CLIPB10 regulates melanization by promoting the activation of proPO, we characterized the molecular functions of CLIPB10 using biochemical approaches. The annotated full-length coding sequence of CLIPB10 encodes a 362 amino acid long protein with a predicted 19 amino acid long signal peptide at the $\mathrm{N}$ terminus. The mature protein contains a single, canonical type 2 clip domain signature of $\mathrm{C}-\mathrm{X}_{9}-\mathrm{C}-\mathrm{X}_{5}-\mathrm{C}-\mathrm{X}_{26}-\mathrm{C}-\mathrm{X}_{7}-\mathrm{CC}$ at the $\mathrm{N}$ terminus, and a S1A protease domain with the conserved $\mathrm{H}-\mathrm{D}-\mathrm{S}$ catalytic triad at the C-terminus. These two domains are separated by a linker region, which contains the LADR 

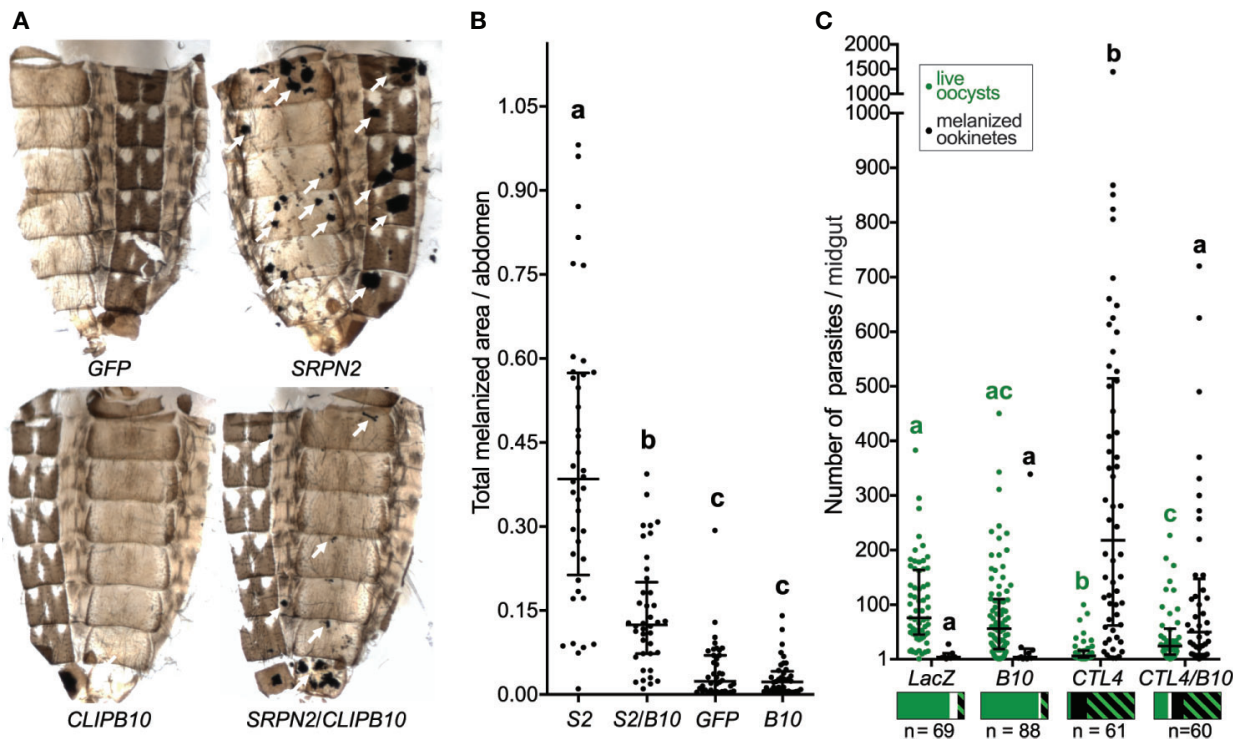

FIGURE 1 | CLIPB10 knockdown partially reverts dsSRPN2- and dsCTL4-induced phenotypes. (A) Abdominal images were collected 21 days post dsRNA injection. Melanotic tumors are indicated by white arrows. (B) Total melanotic areas per abdomen (arbitrary unit) were quantified by Image J. Median and interquartile range are marked $(n=40)$. Kruskal-Wallis test followed by Dunn's multiple comparison test were performed to calculate statistical significance. Medians with a common letter are not significantly different $(P>0.05)$. (C) Live GFP-expressing oocysts (green circles) and melanized ookinetes (black circles) per gut were scored. Median and interquartile range are marked ( $n$, number of guts examined per treatment group). Both oocyst and melanized ookinete infection intensities, respectively were statistically significantly different between all treatment groups (Kruskal-Wallis test $P<0.0001$ ). Medians with a common letter are not significantly different (Dunn's multiple comparison test, $P>0.05$ ), with letters in green indicating live oocyst comparisons, and letters in black indicating melanized ookinetes (see Table S3 for complete summary statistics). The bar charts below each treatment group show prevalence as the fraction of guts containing live oocysts only (green), melanized ookinetes only (black), both live oocysts and melanized ookinetes (green/black hatch), and no parasites (white). The statistical analyses of the prevalence data are summarized in Table S4

putative activation cleavage site. Since CLIPB10 is expressed as a zymogen, and its endogenous activating protease is currently unknown, we expressed a recombinant, mutated version of the proCLIPB10 that enables its cleavage in vitro by commercially available bovine Factor Xa. SDS-PAGE analysis of purified recombinant proCLIPB10 $\mathrm{Xa}_{\text {a }}$ reveals a mass of approximately 43 $\mathrm{kDa}$ (Figure S2), which is slightly higher than the predicted molecular weight of $39.1 \mathrm{kDa}$. This difference is likely due to glycosylation, as the protein sequence contains two predicted Nlinked glycosylation sites. Addition of Factor Xa to purified recombinant proCLIPB10 $\mathrm{Xa}$ resulted in the appearance of a band around $38 \mathrm{kDa}$, which matches the predicted size of the catalytic domain of CLIPB10 ${ }_{\mathrm{Xa}}$, indicating that factor $\mathrm{Xa}$ efficiently cleaves proCLIPB $10_{\mathrm{Xa}}$. To identify the optimal artificial substrate for measuring the amidase activity of CLIPB10, we screened eight short chromogenic peptides with activated CLIPB10Xa. Activated CLIPB10 Xa showed amidase activity against peptide substrates with arginine at the P1 site, including IEAR $p$ NA (Figure S3).

\section{Active CLIPB10xa Is Directly Inhibited by Recombinant SRPN2}

Inhibition of proteases by serpins requires the formation of a SDS-stable complex of the serpin and its cognate protease. To determine if CLIPB10 can be inhibited by SRPN2, we first tested whether these two proteins form such complexes in vitro.
Activated CLIPB10 ${ }_{\mathrm{Xa}}$ was incubated with purified recombinant SRPN2 and complex formation was analyzed by SDS-PAGE. When SRPN2 was added to activate CLIPB10 ${ }_{\mathrm{Xa}}$ the $38 \mathrm{kDa}$ band corresponding to the protease domain of CLIPB10 was not detected, and instead, a higher molecular weight band of around $72 \mathrm{kDa}$ was observed, which matched the predicted molecular weight of a CLIPB10 ${ }_{\mathrm{Xa}}: \mathrm{rSRPN} 2$ inhibitory complex (Figure 2A). Additionally, a second higher molecular weight band of $55 \mathrm{kDa}$ appeared, which likely constitutes a partially degraded form of the inhibitory complex (Figure 2A). Analysis of tryptic peptides from both the $72-$ and $55-\mathrm{kDa}$ bands by ESIMS identified both SRPN2 and CLIPB10 in both bands, confirming the formation of covalent complexes between CLIPB10 $\mathrm{Xa}$ and rSRPN2 (Figure S4). To confirm that this complex formation indeed leads to inhibition of CLIPB10, we tested the ability of SRPN2 to inhibit the IEARase activity of activated CLIPB $10_{\mathrm{Xa}}$ in vitro. CLIPB10 activity decreased linearly with increasing concentrations of SRPN2 (Figure 2B). The stoichiometry of inhibition was 1.89 , indicating that, under the used experimental conditions, SRPN2 acts as an inhibitor and not as substrate for CLIPB10.

\section{CLIPB10 $x a$ Promotes proPO Cleavage and Activation in Manduca sexta Plasma}

The biochemical analysis of proPO activation in mosquitoes is hindered by the limited amount of hemolymph that can be 

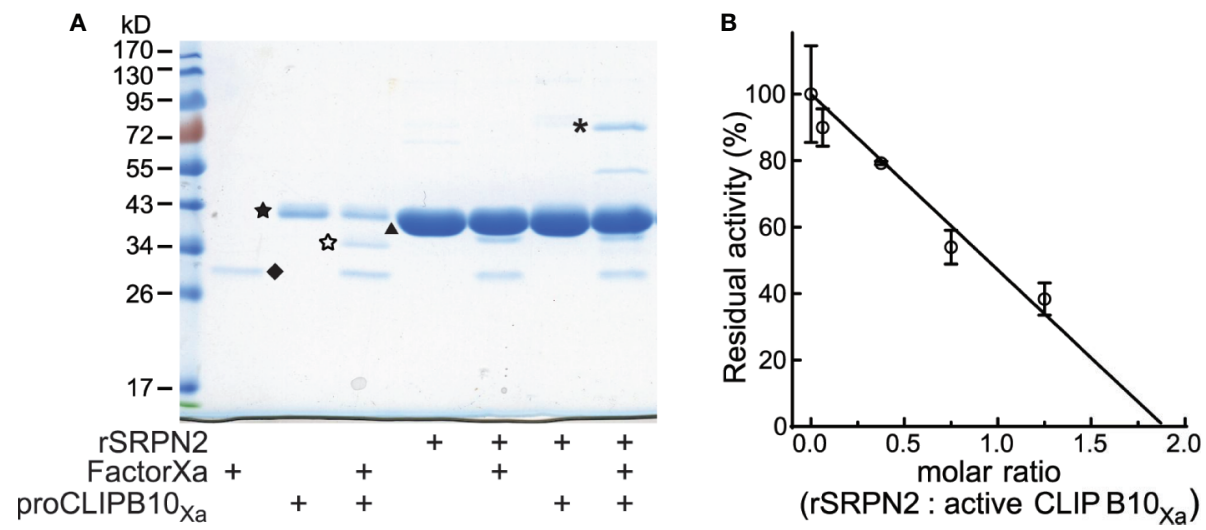

FIGURE 2 | Active CLIPB10xa is inhibited by rSRPN2. (A) SDS-PAGE and Coomassie blue staining shows the presence of a covalent complex formed by rCLIPB10 $x_{a}$ and rSRPN2, indicated by asterisk. CLIPB10 $x_{a}$ zymogen is indicated by a filled star, catalytic domain is indicated by an open star, factor Xa is indicated by diamond, and rSRPN2 is indicated by triangle. (B) IEARpNa was used as the substrate to measure the residual amidase activity of active CLIPB10xa, which was inhibited by rSRPN2 at increasing molar ratios. Data are shown as means \pm S.D. $(n=3)$. The stoichiometry of inhibition is 1.89 .

extracted. This shortage can be overcome by using the $M$. sexta model system as a source of proPO (Michel et al., 2006; An et al., 2011; Zhang et al., 2016). We used plasma from M. sexta larvae to explore the impact of CLIPB10 on the proPO activation cascade. $M$. sexta plasma samples were pre-screened as previously described (Tong and Kanost, 2005). Activated CLIPB10 Xa was incubated with $M$. sexta plasma, followed by western blot analysis using anti-M. sexta PO antibody. A doublet band around $80 \mathrm{kDa}$ in $M$. sexta plasma represents heterodimeric proPO consisting of $79-\mathrm{kDa}$ proPO1 and $80-$ $\mathrm{kDa}$ proPO2 (Jiang et al., 1997). Addition of activated CLIPB10 Xa to $M$. sexta plasma resulted in the appearance of a $70-\mathrm{kDa}$ doublet band corresponding to $M$. sexta active PO (Figure 3A), and an additional band around $55 \mathrm{kD}$ of unknown identity. The same doublet band was observed in the plasma supplemented with activated CLIPB9 Xa, which we identified previously as a functional PAP in An. gambiae (An et al., 2011). PO activity of plasma increased in the presence of active CLIPB9 ${ }_{\mathrm{Xa}}$, and increased even more in the presence of active CLIPB10 $\mathrm{Xa}$ (Figure 3B). These results confirm that the proteolytic activity of CLIPB10 promotes proPO cleavage and PO activity.

\section{CLIPB10 $x$ Functions as a Prophenoloxidase Activating Protein}

To determine the placement of CLIPB10 in the proPO activation cascade, we used a targeted approach to identify nascent protein substrates of CLIPB10 using recombinant proteins. We first tested whether CLIPB10 promotes PO activity by activating proCLIPB9, the only known terminal protease of the proPO activation cascade in An. gambiae. To test whether CLIPB10 can

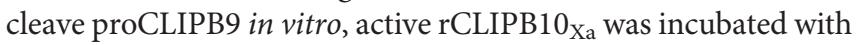
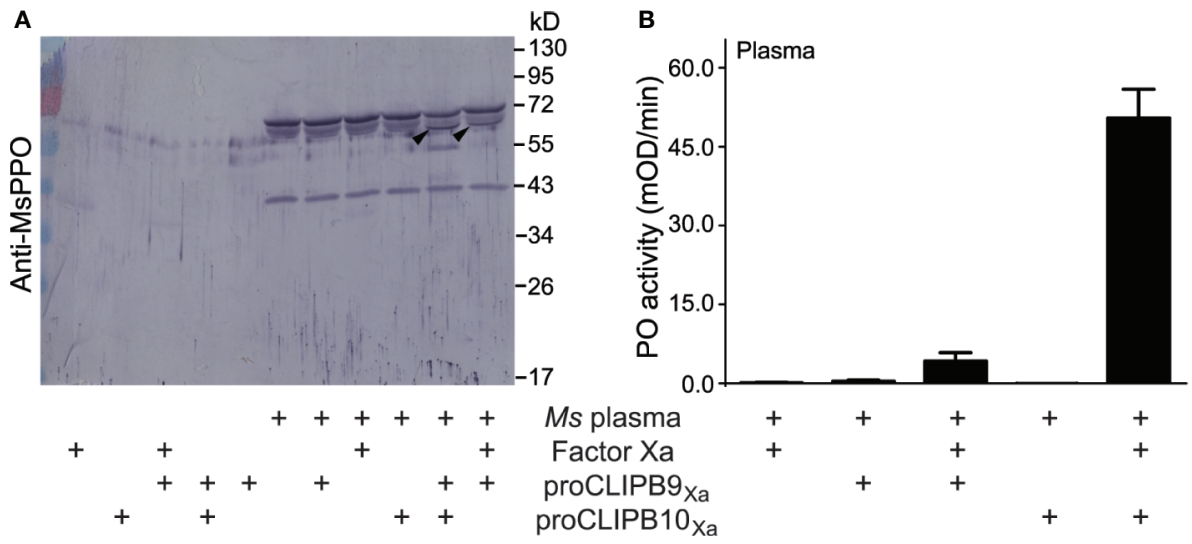

FIGURE 3 | Plasma proPO is cleaved and activated by active rCLIPB10 $x_{a}$. (A) Purified recombinant proCLIPB9xa or proCLIPB10xa were activated by Factor Xa, and was added individually to $M$. sexta plasma. The Western blot of the reactions was probed with anti-M. sexta PPO antibody. Black arrows indicate the band that correspond to $M$. sexta PO. (B) PO activity was observed when plasma was supplemented with activated CLIPB9xa or CLIPB10 $x_{x}$. Data are shown as means \pm S.D $(n=6)$. 
recombinant proCLIPB9, followed by western blot analysis using either anti-His antibody or anti-CLIPB9 antibody. Addition of active CLIPB10 ${ }_{\mathrm{Xa}}$ did not result in the cleavage of proCLIPB9 (Figure 4A), suggesting that CLIPB10 is not directly upstream of CLIPB9. Addition of active CLIPB $8_{\mathrm{Xa}}$ also did not lead to cleavage of proCLIPB9, as reported previously (Zhang et al., 2016).

We next tested whether CLIPB10 promotes PO activity by activating proCLIPB8, a protease required for tissue melanization, which is upstream of CLIPB9 in the proPO activation cascade in An. gambiae. To test whether CLIPB10 can cleave proCLIPB8 in vitro, active rCLIPB10 ${ }_{\mathrm{Xa}}$ was incubated with recombinant proCLIPB8, followed by western blot analysis using either anti-His antibody or anti-CLIPB9 antibody. Addition of active CLIPB10 $0_{\mathrm{Xa}}$ did not result in the cleavage of proCLIPB9 (Figure 4B), suggesting that CLIPB10 is not directly upstream of CLIPB9.

To explore whether CLIPB10 directly catalyzes proPO activation, active CLIPB10 Xa was incubated with purified $M$. sexta proPO, followed by western blot analysis using anti- $M$. sexta $\mathrm{PO}$ antibody. Heterodimeric $M$. sexta proPO remained intact after incubation with Factor Xa, proCLIPB $10_{\mathrm{Xa}}$, and proCLIPB9 ${ }_{\mathrm{Xa}}$, respectively. In contrast, both active CLIPB10 and CLIPB9 ${ }_{\text {Хa }}$ were able to cleave $M$. sexta proPO in vitro, as demonstrated by the appearance of a doublet band corresponding to $M$. sexta PO (Figure 4C). These data demonstrate that CLIPB10, similar to CLIPB9, can function as a PAP in insect hemolymph.

\section{DISCUSSION}

To investigate the complexity of immune regulation in $A n$. gambiae, this study examined the molecular make-up of the protease cascades that control melanization via proteolytic proPO activation. Specifically, this study set out to answer the following two questions: Firstly, do parallel proPO activation cascades exist in An. gambiae, as they do in other insect species, including M. sexta (Jiang et al., 1998; Jiang et al., 2003a; Jiang et al., 2003b). Secondly, are separate proPO activation cascades involved in tissue and humoral melanization as suggested in Ae. aegypti (Zou et al., 2010). To address these questions, we focused our analysis on CLIPB10, based on the results of previous targeted reverse genetic screens and phylogenetic analyses of CLIPBs performed by us and others (Paskewitz et al., 2006; An et al., 2011; Cao et al., 2017).

To answer the first question, we examined the molecular function of CLIPB10 by biochemical means. The hallmark of parallel proPO activation cascades is the existence of two or more PAPs that independently cleave and activate proPO, and crosstalk between cascades is observed at levels upstream of the terminal PAP (e.g., An et al., 2009; Wang Y. et al., 2020). We posited a role of CLIPB10 as a PAP based on the following existing data. Previously, we had identified the first PAP, CLIPB9 (AGAP029769) and its inhibitor, SRPN2 (AGAP006911) in An. gambiae (An et al., 2011). Our analysis of the phylogenetic relationships among An. gambiae CLIPB proteases clustered CLIPB9 and CLIPB10 (AGAP029770) with M. sexta PAP1 and D. melanogaster MP2, to the exclusion of other An. gambiae, M. sexta and D. melanogaster sequences. Given that CLIPB9, PAP1 and MP2 are known terminal proteases in proPO activation cascades, CLIPB10 was therefore an excellent candidate for PAP function in An. gambiae (Jiang et al., 1998; Tang et al., 2006; An et al., 2013). Using recombinant protein expressed with the baculovirus expression system, we obtained active CLIPB10 protein in vitro. Activated CLIPB10 added to $M$. sexta plasma results in significant activation cleavage of proPO and $\mathrm{PO}$ activity. Activated CLIPB10 also resulted in activation cleavage of purified $M$. sexta proPO. Therefore, CLIPB10 can function as the terminal protease in the proPO activation cascade. Given that seven out of eight proPOs of An. gambiae share the same activation cleavage site with $M$. sexta proPO (Jiang et al., 2003a; Michel et al., 2005), the data presented here strongly suggest that CLIPB10 functions as a PAP in the hemolymph of An. gambiae mosquitoes.

We previously showed that SPRN2 functions as a master regulator of melanization in mosquitoes, by inhibiting the PAP function of CLIPB9 (An et al., 2011). In this current study, we show that SRPN2 also inhibits activated CLIPB10 in vitro, as demonstrated by the formation of inhibitory protease-serpin complexes, and the reduction of CLIPB10's amidase activity with increasing molar ratios of SRPN2 to CLIPB10 in vitro. This interaction of CLIPB10 and SRPN2 also occurs in vivo. The total area of melanized tumors in the abdomen of SRPN2 kd mosquitoes was decreased by $65 \%$ when CLIPB10 kd was also invoked. Together, these results demonstrate the existence of two PAPs in An. gambiae, and suggests that two proPO activation cascades exist in this mosquito species. This parallels the findings in $M$. sexta, where two separate proPO activation cascades result in the activation of PAP1 and PAP2/ PAP3, respectively (Jiang et al., 1998; Jiang et al., 2003a; Jiang et al., 2003b). The activity of terminal PAPs in both proPO activation cascades in $M$. sexta are inhibited by the same serpin, Serpin-3 (Zhu et al., 2003; Christen et al., 2012). We find the same to be true for the two putative proPO activation cascades in An. gambiae. SRPN2, the ortholog of M. sexta Serpin-3 inhibits both CLIPB9 and CLIPB10 with similar stoichiometry of inhibition (1.3 for SRPN2:CLIPB9, and 1.7 for SRPN2: CLIPB10), indicating both PAPs are subjected to efficient SRPN2 sequestration. Beyond being inhibited by the same serpin, we thus far have found no evidence for additional interactions between the CLIPB9 and B10 PAPs, as recombinant activated CLIPB10 did not activate CLIPB9 in vitro. Preliminary triple knockdown analyses suggests additive effects between the two PAPs, as double kd of CLIPB9 and B10 further reduced melanotic tumour formation due to SRPN2 depletion (Zhang and Michel, unpublished). However, epistasis analyses using RNAi are limited by the incomplete knockdown of CLIPB9, and thus will be explored in future analyses beyond the scope of this current study.

To address the second question of whether separate proPO activation cascades lead to tissue and humoral melanization in 

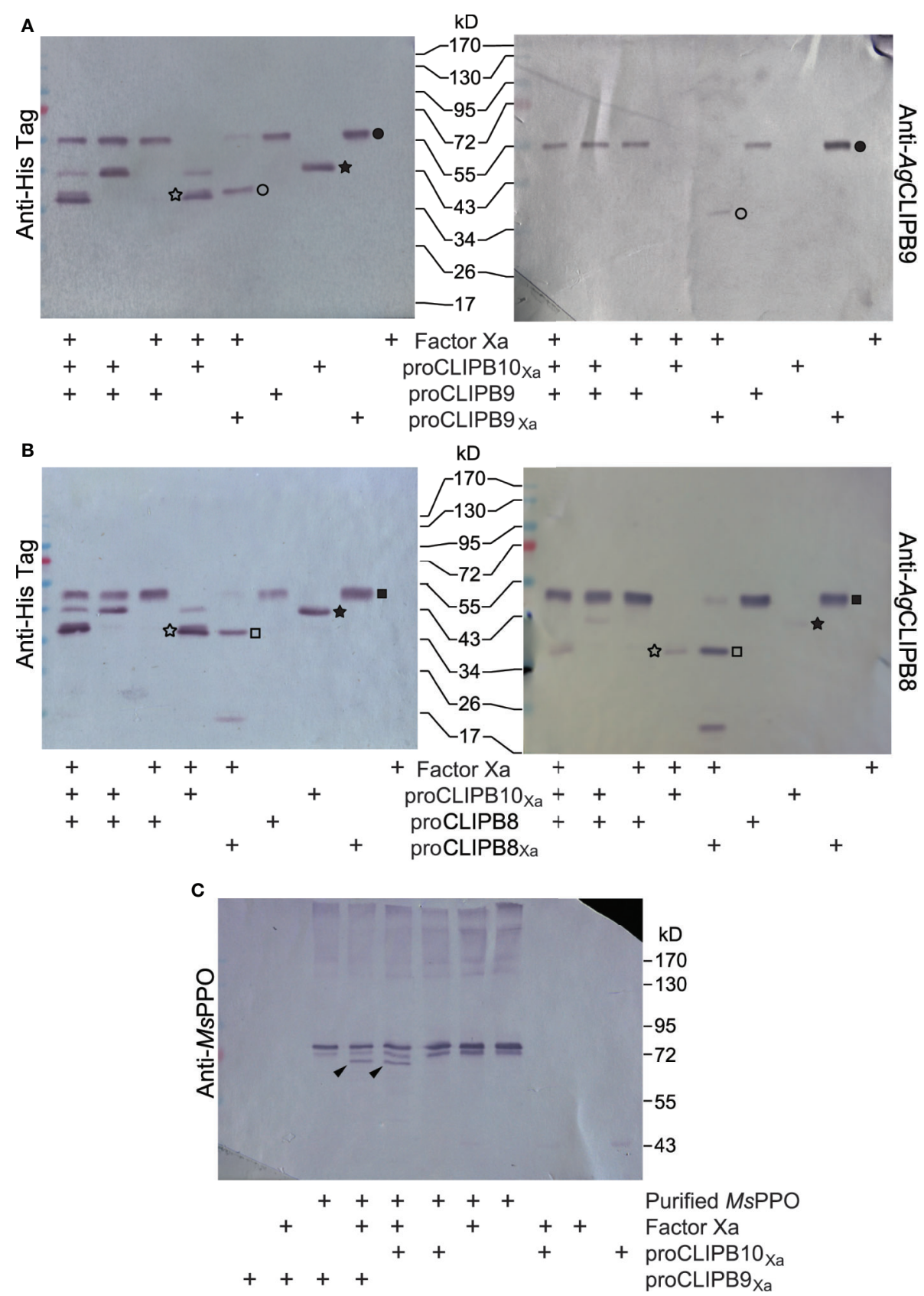

FIGURE 4 | Placement of CLIPB10 in the proPO activation cascade. (A) Neither activated rCLIPB10xa, nor commercial Factor Xa cleave recombinant wild-type proCLIPB9. (B) Neither activated rCLIPB10 xa, nor commercial Factor Xa cleave recombinant wild-type proCLIPB8. All recombinant proteins contain a His-tag at their C-terminus, which is detected by anti-His antibody (left panels), and are also recognized by their specific antibodies. Filled star, proCLIPB10xa; open star, protease domain of rCLIPB10 $x_{a}$; filled circle, zymogen form of the recombinant proCLIPB9 and proCLIPB9 $x_{a}$, open circle, protease domain of rCLIPB9xa; filled square, zymogen form of the recombinant proCLIPB8 and proCLIPB8 xa; open square, protease domain of rCLIPB8 $x a$. (C) Activated rCLIPB10xa directly cleaves M. sexta proPO. Black arrows indicate a smaller PO band on Western blots probed with anti-M. sexta PPO antibody.

An. gambiae, we examined the function of CLIPB10 in two distinct models of melanization in An. gambiae. The study by Paskewitz et al. had implicated CLIPB10 in the humoral melanization of Sephadex beads in the hemocoel of adult female An. gambiae (Paskewitz et al., 2006), as CLIPB10 kd resulted in a small but significant reduction in the number of Sephadex beads that in control mosquitoes were $100 \%$ melanized after overnight incubation. Our analysis of the role of CLIPB10 in CTL4 kd-mediated melanization of rodent $P$. berghei parasites further supports the role of CLIPB10 in 
humoral melanization. CLIPB10/CTL4 double-kd reduced the average number of melanized ookinetes per midgut by $77 \%$ as compared to CTL4 $\mathrm{kd}$ mosquitoes. While CLIPB10 kd quadrupled the number of live oocysts as compared to CTL4 $\mathrm{kd}$, live oocyst numbers remained one third of those observed in control mosquitoes. Thus, CLIPB10 is largely required for melanin deposition on the parasite surface and to a lesser degree impacts the parasite killing observed in CTL4 $\mathrm{kd}$ mosquitoes. Taken together, these data clearly demonstrate that CLIPB10 is required for humoral melanization of parasites and to a lesser extent Sephadex beads, as well as tissue melanization. This is in contrast to findings in Ae. aegypti, were two distinct regulatory modules of tissue melanization and hemolymph proPO activation were described (Zou et al., 2010). Tissue melanization in Ae. aegypti requires two cSPs, TMP, the ortholog of An. gambiae CLIPB8, and IMP-1, the ortholog of An. gambiae CLIPB9, and is inhibited by serpin-2, the ortholog of An. gambiae SRPN2. In contrast, humoral melanization, which the authors analyze through the cleavage of proPO in the hemolymph, is inhibited by serpin-1 and serpin-3, and requires the action of IMP-1 as well as IMP-2. In contrast, our results suggest that in An. gambiae, proPO activation cleavage is mediated by both CLIPB9 and CLIPB10, and inhibited directly by SRPN2. Our data strongly suggest that the difference between humoral and tissue melanization in An. gambiae is not due to the action of different proPO activation cascades. Instead, tissue melanization is the consequence of dysregulated melanogenesis due to constitutively active proPO activation cascades induced by SRPN2 kd. This dysregulated melanogenesis is also exemplified by the dramatic activation of key regulators of humoral melanization, such as CLIPA8, CLIPA28, and CLIPA14, in the hemolymph of naive SRPN2 kd mosquitoes (El Moussawi et al., 2019). Melanogenic reactions must occur highly localized on the surface of target entities to prevent adverse impact on selfentities, as unregulated melanogenesis produces harmful byproducts and intermediates that can disperse through hemolymph circulation and result in systemic damage (Nappi and Christensen, 2005). In SRPN2 kd mosquitoes, this systemic damage is not only visualized by tissue melanization, but can also be measured by concomitant reduced lifespan (Michel et al., 2005; Volz et al., 2005; Volz et al., 2006; An et al., 2011; Zhang et al., 2016). While the localization of melanization on microbial surfaces is achieved by the opsonizing function of TEP1 (Blandin et al., 2004; Yassine et al., 2012a; Povelones et al., 2013), future studies will have to determine whether TEP1 and/ or other components of the complement pathway are also required for tissue melanization.

Both CLIPB9 and CLIPB10 are secreted as zymogens into the hemolymph of An. gambiae, and require activation cleavage. However, their nascent activating proteases are currently unknown. Studies in other model insects have shown that PAP activation cleavage is mediated by cSPs belonging to the CLIPC family, which in turn are proteolytically activated by an upstream modular serine protease (ModSP). Examples of such proPO activation cascades have been described in several model organisms, including M. sexta (Gorman et al., 2007; Wang and Jiang, 2007), Tenebrio molitor (Kan et al., 2008; Jiang et al., 2009), and most recently Helicoverpa armigera (Wang Q. et al., 2020). While currently no data exist that show activation cleavage of either CLIPB9 or CLIPB10 by an endogenous CLIPC, recent genetic evidence suggests that CLIPC9 is required for humoral melanization of parasites as well as tissue melanization (Sousa et al., 2020). Concomitant knockdown of CTL4 and CLIPC9 reversed ookinete melanization without rescuing parasite killing, thus phenocopying the CTL4/CLIPB10 double-kd. CLIPC9 is thus an excellent candidate for a PAP-activating protease in $A n$. gambiae, and a potential activator of CLIPB10 during infectioninduced melanization. The data provided by Sousa and coauthors also suggest that CLIPC9 undergoes localized activation cleavage, binding to microbial surfaces in a cleaved form (Sousa et al., 2020). The cleavage of CLIPC9 depends on components of the complement-like pathway, but the nascent protease mediating CLIPC9 cleavage awaits identification. A ModSP, which potentially could function as a CLIPC9activating protease is SP217. SP217 has a similar domain structure to $M$. sexta HP14, the only known ModSP to function in proPO activation upstream of PAP1/PAP2 (Wang and Jiang, 2007). Addition of recombinant SP217 to M. sexta plasma increased PO activity, suggesting that SP217 may substitute HP14 function (Wang Y. et al., 2020). This notion is further supported by our finding that SP217 kd significantly reduced SRPN2 kd-mediated melanotic tumor formation (Zhang and Michel, unpublished). Future studies will have to determine whether SP217, CLIPC9, and CLIPB9 or B10 indeed constitute proPO activation cascades in An. gambiae.

In summary, our study demonstrates that CLIPB10 is the second prophenoloxidase-activating enzyme identified in $A n$. gambiae, in addition to the previously identified CLIPB9, suggesting functional redundancy in the cSP network that controls melanization. In addition, our data suggest that tissue melanization and humoral melanization are at least partially mediated by the same CLIPB proteases. Studies are currently underway to determine whether additional PAPs exist, and in how far these parallel proPO activation cascades provide true functional redundancy or are evoked non-redundantly dependent on the immune challenge.

\section{DATA AVAILABILITY STATEMENT}

The original contributions presented in the study are included in the article/Supplementary Materials. Further inquiries can be directed to the corresponding author.

\section{ETHICS STATEMENT}

The animal study was reviewed and approved by the Institutional Animal Care and Use committee IACUC of the American University of Beirut. 


\section{AUTHOR CONTRIBUTIONS}

XZ, LM, SS, and SZ performed the experiments and analyzed the data. KM and ML drafted the manuscript. XZ, MO, and KM designed the experiments. All authors contributed to the article and approved the submitted version.

\section{FUNDING}

This study was supported by funding from the National Institutes of Health grant numbers R01AI095842 and R01AI140760, and the USDA National Institute of Food and Agriculture, Hatch project 1021223 to KM. This is contribution no. 21-011-J from the Kansas Agricultural Experiment Station. Its contents are solely the responsibility of the authors and do not necessarily represent the official views of the funding agencies.

\section{REFERENCES}

An, C., Budd, A., Kanost, M. R., and Michel, K. (2011). Characterization of a regulatory unit that controls melanization and affects longevity of mosquitoes. Cell. Mol. Life Sci. 68, 1929-1939. doi: 10.1007/s00018-010-0543-Z

An, C., Ishibashi, J., Ragan, E. J., Jiang, H., and Kanost, M. R. (2009). Functions of Manduca sexta hemolymph proteinases HP6 and HP8 in two innate immune pathways. J. Biol. Chem. 284, 19716-19726.

An, C., Zhang, M., Chu, Y., and Zhao, Z. (2013). Serine protease MP2 activates prophenoloxidase in the melanization immune response of Drosophila melanogaster. PloS One 8, e79533. doi: 10.1371/journal.pone.0079533

Bartholomay, L. C., and Michel, K. (2018). Mosquito Immunobiology: The Intersection of Vector Health and Vector Competence. Annu. Rev. Entomol. 63, 145-167. doi: 10.1146/annurev-ento-010715-023530

Blandin, S., Shiao, S. H., Moita, L. F., Janse, C. J., Waters, A. P., Kafatos, F. C., et al. (2004). Complement-like protein TEP1 is a determinant of vectorial capacity in the malaria vector Anopheles gambiae. Cell 116, 661-670. doi: 10.1016/ S0092-8674(04)00173-4

Brey, P. T., Lebrun, R. A., Papierok, B., Ohayon, H., Vennavalli, S., and Hafez, J. (1988). Defense reactions by larvae of Aedes aegypti during infection by the aquatic fungus Lagenidium giganteum (Oomycete). Cell Tissue Res. 253, 245250. doi: $10.1007 / \mathrm{BF} 00221760$

Cao, X., Gulati, M., and Jiang, H. (2017). Serine protease-related proteins in the malaria mosquito, Anopheles gambiae. Insect Biochem. Mol. Biol. 88, 48-62. doi: 10.1016/J.IBMB.2017.07.008

Cerenius, L., and Soderhall, K. (2004). The prophenoloxidase-activating system in invertebrates. Immunol. Rev. 198, 116-126. doi: 10.1111/j.0105-2896.2004.00116.x

Chen, C. C., and Laurence, B. R. (1987). Selection of a Strain of AnophelesQuadrimaculatus with High Filaria Encapsulation Rate. J. Parasitol. 73, 418-419.

Christen, J. M., Hiromasa, Y., An, C., and Kanost, M. R. (2012). Identification of plasma proteinase complexes with serpin-3 in Manduca sexta. Insect Biochem. Mol. Biol. 42, 946-955. doi: 10.1016/j.ibmb.2012.09.008

Christensen, B. M. (1981). Observations on the immune response of Aedes trivittatus against Dirofilaria immitis. Trans. R. Soc. Trop. Med. Hyg. 75, 439-443. doi: 10.1016/0035-9203(81)90116-4

Christophides, G. K., Zdobnov, E., Barillas-Mury, C., Birney, E., Blandin, S., Blass, C., et al. (2002). Immunity-related genes and gene families in Anopheles gambiae. Science 298, 159-165. doi: 10.1126/science.1077136

Chun, J., Riehle, M., and Paskewitz, S. M. (1995). Effect of mosquito age and reproductive status on melanization of sephadex beads in Plasmodiumrefractory and -susceptible strains of Anopheles gambiae. J. Invertebr. Pathol. 66, 11-17. doi: 10.1006/jipa.1995.1054

Collins, F. H., Sakai, R. K., Vernick, K. D., Paskewitz, S., Seeley, D. C., Miller, L. H., et al. (1986). Genetic selection of a Plasmodium-refractory strain of the malaria vector Anopheles gambiae. Science 234, 607-610. doi: 10.1126/science.3532325

\section{ACKNOWLEDGMENTS}

We thank Maureen Gorman (Kansas State University) for purified M. sexta proPO, Connor Hartpence for the cloning of CLIPB10, all members of the Michel laboratory for mosquito rearing, and J. Tomich at the Kansas State Biotechnology/Proteomics Core Lab for the ESI-MS analyses. In addition, we thank the Kamal A. Shair Central Research Science Laboratory at AUB for providing free access to its equipment.

\section{SUPPLEMENTARY MATERIAL}

The Supplementary Material for this article can be found online at: https://www.frontiersin.org/articles/10.3389/fcimb.2020.585986/ full\#supplementary-material

De Gregorio, E., Han, S.-J., Lee, W.-J., Baek, M.-J., Osaki, T., Kawabata, S.-I., et al. (2002). An immune-responsive Serpin regulates the melanization cascade in Drosophila. Dev. Cell 3, 581-592. doi: 10.1016/s1534-5807(02)00267-8

El Moussawi, L., Nakhleh, J., Kamareddine, L., and Osta, M. A. (2019). The mosquito melanization response requires hierarchical activation of noncatalytic clip domain serine protease homologs. PloS Pathog. 15, e1008194. doi: 10.1371 /journal.ppat.1008194

Franke-Fayard, B., Trueman, H., Ramesar, J., Mendoza, J., van der Keur, M., van der Linden, R., et al. (2004). A Plasmodium berghei reference line that constitutively expresses GFP at a high level throughout the complete life cycle. Mol. Biochem. Parasitol. 137, 23-33. doi: 10.1016/j.molbiopara. 2004.04.007

Frolet, C., Thoma, M., Blandin, S., Hoffmann, J. A., and Levashina, E. (2006). Boosting NF- $\mathrm{BB}$-dependent basal immunity of Anopheles gambiae aborts development of Plasmodium berghei. Immunity 25, 677-685. doi: 10.1016/ j.immuni.2006.08.019

Gettins, P. G. W. (2002). Serpin structure, mechanism, and function. Chem. Rev. 102, 4751-4804. doi: 10.1021/cr010170+

Gorman, M. J., Wang, Y., Jiang, H., and Kanost, M. R. (2007). Manduca sexta hemolymph proteinase 21 activates prophenoloxidase-activating proteinase 3 in an insect innate immune response proteinase cascade. J. Biol. Chem. 282, 11742-11749. doi: 10.1074/jbc.M611243200

Habtewold, T., Povelones, M., Blagborough, A. M., and Christophides, G. K. (2008). Transmission Blocking Immunity in the Malaria Non-Vector Mosquito Anopheles quadriannulatus Species A. PloS Pathog. 4, e1000070. doi: 10.1371/journal.ppat.1000070

Hillyer, J. F., Schmidt, S. L., and Christensen, B. M. (2003). Hemocyte-mediated phagocytosis and melanization in the mosquito Armigeres subalbatus following immune challenge by bacteria. Cell Tissue Res. 313, 117-127. doi: 10.1007/s00441-003-0744-y

Hurd, H., Taylor, P. J., Adams, D., Underhill, A., and Eggleston, P. (2005). Evaluating the costs of mosquito resistance to malaria parasites. Evol. Int. J Organic Evol. 59, 2560-2572. doi: 10.1554/05-211.1

Jiang, H., Wang, Y., Ma, C., and Kanost, M. R. (1997). Subunit Composition of Pro-phenol Oxidase from Manduca sexta: Molecular Cloning of Subunit ProPO-p1. Insect Biochem. Mol. Biol. 27, 835-850. doi: 10.1016/s0965-1748 (97)00066-0

Jiang, H., Wang, Y., and Kanost, M. R. (1998). Pro-phenol oxidase activating proteinase from an insect, Manduca sexta: a bacteria-inducible protein similar to Drosophila easter. Proc. Natl. Acad. Sci. U. S. A. 95, 12220-12225. doi: 10.1073/pnas.95.21.12220

Jiang, H., Wang, Y., Yu, X. Q., and Kanost, M. R. (2003a). Prophenoloxidaseactivating proteinase-2 from hemolymph of Manduca sexta. A bacteriainducible serine proteinase containing two clip domains. J. Biol. Chem. 278, 3552-3561. doi: 10.1074/jbc.M205743200 
Jiang, H., Wang, Y., Yu, X. Q., Zhu, Y., and Kanost, M. (2003b). Prophenoloxidase-activating proteinase-3 (PAP-3) from Manduca sexta hemolymph: a clip-domain serine proteinase regulated by serpin-1J and serine proteinase homologs. Insect Biochem. Mol. Biol. 33, 1049-1060. doi: 10.1016/S0965-1748(03)00123-1

Jiang, R., Kim, E. H., Gong, J. H., Kwon, H. M., Kim, C. H., Ryu, K. H., et al. (2009). Three pairs of protease-serpin complexes cooperatively regulate the insect innate immune responses. J. Biol. Chem. 284, 35652. doi: 10.1074/ jbc.M109.071001

Kan, H., Kim, C.-H. H., Kwon, H.-M. M., Park, J.-W. W., Roh, K.-B. B., Lee, H., et al. (2008). Molecular control of phenoloxidase-induced melanin synthesis in an insect. J. Biol. Chem. 283, 25316. doi: 10.1074/jbc.M804364200

Kanost, M. R., and Jiang, H. (2015). Clip-domain serine proteases as immune factors in insect hemolymph. Curr. Opin. Insect Sci. 11, 47-55. doi: 10.1016/ j.cois.2015.09.003

Law, R. H., Zhang, Q., McGowan, S., Buckle, A. M., Silverman, G. A., Wong, W., et al. (2006). An overview of the serpin superfamily. Genome Biol. 7, 216. doi: 10.1186/gb-2006-7-5-216

Ligoxygakis, P. (2002). A serpin mutant links Toll activation to melanization in the host defence of Drosophila. EMBO J. 21, 6330-6337. doi: 10.1093/emboj/ cdf661

Meekins, D. A., Kanost, M. R., and Michel, K. (2016). Serpins in Arthropod Biology. Semin. Cell Dev. Biol. 62, 105-119. doi: 10.1016/j.semcdb.2016.09.001

Michel, K., Budd, A., Pinto, S., Gibson, T. J., and Kafatos, F. C. (2005). Anopheles gambiae SRPN2 facilitates midgut invasion by the malaria parasite Plasmodium berghei. EMBO Rep. 6, 891-897. doi: 10.1038/ sj.embor. 7400478

Michel, K., Suwanchaichinda, C., Morlais, I., Lambrechts, L., Cohuet, A., AwonoAmbene, P. H., et al. (2006). Increased melanizing activity in Anopheles gambiale does not affect development of Plasmodium falciparum. Proc. Natl. Acad. Sci. U. States America 103, 16858-16863. doi: 10.1073/pnas.0608033103

Nakhleh, J., Christophides, G. K., and Osta, M. A. (2017a). The serine protease homolog CLIPA14 modulates the intensity of the immune response in the mosquito Anopheles gambiae. J. Biol. Chem. 292, 18217-18226. doi: 10.1074/ jbc.M117.797787

Nakhleh, J., El Moussawi, L., and Osta, M. A. (2017b). "The Melanization Response in Insect Immunity," in Advances in Insect Physiology. Ed. P. Ligoxygakis (Cambridge, MA: Academic Press), 83-109. doi: 10.1016/bs.aiip. 2016.11.002

Nappi, A., Poirié, M., Carton, Y., Poirie, M., and Carton, Y. (2009). The role of melanization and cytotoxic by-products in the cellular immune responses of Drosophila against parasitic wasps. Adv. Parasitol. 70, 99-121. doi: 10.1016/ S0065-308X(09)70004-1

Nappi, A. J., and Christensen, B. M. (2005). Melanogenesis and associated cytotoxic reactions: Applications to insect innate immunity. Insect Biochem. Mol. Biol. 35, 443-459. doi: 10.1016/j.ibmb.2005.01.014

Osta, M. A., Christophides, G. K., and Kafatos, F. C. (2004). Effects of mosquito genes on Plasmodium development. Sci. (New York N.Y.) 303, 2030-2032. doi: 10.1126/science.1091789

Paskewitz, S. M., Andreev, O., and Shi, L. (2006). Gene silencing of serine proteases affects melanization of Sephadex beads in Anopheles gambiae. Insect Biochem. Mol. Biol. 36, 701-711. doi: 10.1016/j.ibmb.2006.06.001

Pavan, M. E., López, N.II, and Pettinari, M. J. (2020). Melanin biosynthesis in bacteria, regulation and production perspectives. Appl. Microbiol. Biotechnol. 104, 1357-1370. doi: 10.1007/s00253-019-10245-y

Povelones, M., Bhagavatula, L., Yassine, H., Tan, L. A., Upton, L. M., Osta, M. A., et al. (2013). The CLIP-Domain Serine Protease Homolog SPCLIP1 Regulates Complement Recruitment to Microbial Surfaces in the Malaria Mosquito Anopheles gambiae. PloS Pathog. 9, e1003623. doi: 10.1371/journal.ppat. 1003623

Rhodes, V. L. M., and Michel, K. (2017). "Modulation of Mosquito Immune Defenses as a Control Strategy," in Arthropod Vector: Controller of Disease Transmission. Eds. S. K. Wikel, S. Aksoy and G. Dimopoulos (Cambridge, MA: Academic Press) vol. 1., 59-89. doi: 10.1016/B978-0-12-805350-8.00004-0

Rodriguez-Andres, J., Rani, S., Varjak, M., Chase-Topping, M. E., Beck, M. H., Ferguson, M. C., et al. (2012). Phenoloxidase activity acts as a mosquito innate immune response against infection with Semliki Forest virus. PloS Pathog. 8, e1002977. doi: 10.1371/journal.ppat.1002977
Schnitger, A. K. D., Kafatos, F. C., and Osta, M. (2007). The melanization reaction is not required for survival of Anopheles gambiae mosquitoes after bacterial infections. J. Biol. Chem. 282, 21884-21888. doi: 10.1074/jbc.M701635200

Sousa, G. L., Bishnoi, R., Baxter, R. H. G., and Povelones, M. (2020). The CLIPdomain serine protease CLIPC9 regulates melanization downstream of SPCLIP1, CLIPA8, and CLIPA28 in the malaria vector Anopheles gambiae. PloS Pathog. 16, e1008985. doi: 10.1371/journal.ppat.1008985

Sugumaran, M., and Barek, H. (2016). Critical Analysis of the Melanogenic Pathway in Insects and Higher Animals. Int. J. Mol. Sci. 17, 1753. doi: $10.3390 /$ ijms 17101753

Tang, H., Kambris, Z., Lemaitre, B., and Hashimoto, C. (2006). Two Proteases Defining a Melanization Cascade in the Immune System of Drosophila. J. Biol. Chem. 281, 28097-28104. doi: 10.1074/jbc.M601642200

Tong, Y., and Kanost, M. R. (2005). Manduca sexta serpin-4 and serpin-5 inhibit the prophenol oxidase activation pathway: cDNA cloning, protein expression, and characterization. J. Biol. Chem. 280, 14923-14931. doi: 10.1074/jbc. M500531200

Vavricka, C. J., Christensen, B. M., and Li, J. (2010). Melanization in living organisms: a perspective of species evolution. Protein Cell 1, 830-841. doi: 10.1007/s13238-010-0109-8

Volz, J., Osta, M. A., Kafatos, F. C., and Müller, H.-M. (2005). The Roles of Two Clip Domain Serine Proteases in Innate Immune Responses of the Malaria Vector Anopheles gambiae. J. Biol. Chem. 280, 40161-40168. doi: 10.1074/ jbc.M506191200

Volz, J., Muller, H.-M., Zdanowicz, A., Kafatos, F. C., and Osta, M. A. (2006). A genetic module regulates the melanization response of Anopheles to Plasmodium. Cell Microbiol. 8, 1392-1405. doi: 10.1111/j.1462-5822.2006. 00718.x

Wang, Q., Yin, M., Yuan, C., Liu, X., Hu, Z., Zou, Z., et al. (2020). Identification of a Conserved Prophenoloxidase Activation Pathway in Cotton Bollworm Helicoverpa armigera. Front. Immunol. 11, 785. doi: 10.3389/fimmu. 2020.00785

Wang, Y., Yang, F., Cao, X., Huang, R., Paskewitz, S., Hartson, S. D., et al. (2020). Inhibition of immune pathway-initiating hemolymph protease-14 by Manduca sexta serpin-12, a conserved mechanism for the regulation of melanization and Toll activation in insects. Insect Biochem. Mol. Biol. 116, 103261. doi: 10.1016/ j.ibmb.2019.103261

Wang, Y., and Jiang, H. (2007). Reconstitution of a branch of the Manduca sexta prophenoloxidase activation cascade in vitro: Snake-like hemolymph proteinase 21 (HP21) cleaved by HP14 activates prophenoloxidase-activating proteinase-2 precursor. Insect Biochem. Mol. Biol. 37, 1015-1025. doi: 10.1016/ j.ibmb.2007.05.013

Waterhouse, R. M., Kriventseva, E. V., Meister, S., Xi, Z., Alvarez, K. S., Bartholomay, L. C., et al. (2007). Evolutionary Dynamics of Immune-Related Genes and Pathways in Disease-Vector Mosquitoes. Science 316, 1738-1743. doi: $10.1126 /$ science. 1139862

Whisstock, J. C., and Bottomley, S. P. (2006). Molecular gymnastics: serpin structure, folding and misfolding. Curr. Opin. Struct. Biol. 16, 761-768. doi: 10.1016/j.sbi.2006.10.005

Yassine, H., Kamareddine, L., and Osta, M. A. (2012a). The Mosquito Melanization Response Is Implicated in Defense against the Entomopathogenic Fungus Beauveria bassiana. PloS Pathog. 8, e1003029. doi: 10.1371/journal.ppat. 1003029

Yassine, H., Kamareddine, L., and Osta, M. A. (2012b). The mosquito melanization response is implicated in defense against the entomopathogenic fungus Beauveria bassiana. PloS Pathog. 8, e1003029. doi: 10.1371/journal. ppat.1003029

Yassine, H., Kamareddine, L., Chamat, S., Christophides, G. K., and Osta, M. A. (2014). A Serine Protease Homolog Negatively Regulates TEP1 Consumption in Systemic Infections of the Malaria Vector Anopheles gambiae. J. Innate Immun. 6, 806-818. doi: 10.1159/000363296

Zhang, X., An, C., Sprigg, K., and Michel, K. (2016). CLIPB8 is part of the prophenoloxidase activation system in Anopheles gambiae mosquitoes. Insect Biochem. Mol. Biol. 71, 106-115. doi: 10.1016/j.ibmb.2016.02.008

Zhu, Y., Wang, Y., Gorman, M. J., Jiang, H., and Kanost, M. R. (2003). Manduca sexta Serpin-3 Regulates Prophenoloxidase Activation in Response to Infection by Inhibiting Prophenoloxidase-activating Proteinases. J. Biol. Chem. 278, 46556-46564. doi: 10.1074/jbc.M309682200 
Zou, Z., Shin, S. W., Alvarez, K. S., Kokoza, V., and Raikhel, A. S. (2010). Distinct Melanization Pathways in the Mosquito Aedes aegypti. Immunity 32, 41-53. doi: 10.1016/j.immuni.2009.11.011

Conflict of Interest: The authors declare that the research was conducted in the absence of any commercial or financial relationships that could be construed as a potential conflict of interest.
Copyright ( 2021 Zhang, Li, El Moussawi, Saab, Zhang, Osta and Michel. This is an open-access article distributed under the terms of the Creative Commons Attribution License (CC BY). The use, distribution or reproduction in other forums is permitted, provided the original author(s) and the copyright owner(s) are credited and that the original publication in this journal is cited, in accordance with accepted academic practice. No use, distribution or reproduction is permitted which does not comply with these terms. 
OPEN ACCESS

Edited by:

Layla Kamareddine,

Qatar University, Qatar

Reviewed by:

Malgorzata Cytrynska,

Maria Curie-Skłodowska

University, Poland

Arun K. Bhunia,

Purdue University, United States

Olivier Disson,

Institut Pasteur, France

*Correspondence:

Pierre-Yves Donnio

pierre-yves.donnio@univ-rennes1.fr

${ }^{\dagger}$ Deceased author

Specialty section:

This article was submitted to

Bacteria and Host,

a section of the journal

Frontiers in Cellular and

Infection Microbiology

Received: 20 November 2020 Accepted: 26 March 2021

Published: 19 April 2021

Citation:

Ménard G, Rouillon A, Ghukasyan G,

Emily $M$, Felden $B$ and Donnio P-Y

(2021) Galleria mellonella Larvae as an

Infection Model to Investigate

sRNA-Mediated Pathogenesis in

Staphylococcus aureus.

Front. Cell. Infect. Microbiol. 11:631710.

doi: 10.3389/fcimb.2021.631710

\section{Galleria mellonella Larvae as an Infection Model to Investigate sRNA- Mediated Pathogenesis in Staphylococcus aureus}

\author{
Guillaume Ménard ${ }^{1}$, Astrid Rouillon ${ }^{2}$, Gevorg Ghukasyan ${ }^{3}$, Mathieu Emily ${ }^{4}$, \\ Brice Felden ${ }^{2 \dagger}$ and Pierre-Yves Donnio ${ }^{1 *}$
}

1 Univ Rennes, CHU Rennes, INSERM, BRM [Bacterial Regulatory RNAs and Medicine], SB2H (service de Bactériologie Hygiène-Hospitalière), UMR_S 1230, F-35000, Rennes, France, 2 Univ Rennes, INSERM, BRM (Bacterial Regulatory RNAs and Medicine), UMR_S 1230, F-35000, Rennes, France, ${ }^{3}$ Univ Rennes, CNRS, INSERM, BIOSIT (Biologie, Santé, Innovation Technologique de Rennes), UMS 3480, US_S018, F-35000, Rennes, France, ${ }^{4}$ Institut Agro, CNRS, Univ Rennes, IRMAR (Institut de recherche Mathématique de Rennes), UMR 6625, F-35000, Rennes, France

Small regulatory RNAs (sRNAs) are key players in bacterial regulatory networks. Monitoring their expression inside living colonized or infected organisms is essential for identifying sRNA functions, but few studies have looked at sRNA expression during host infection with bacterial pathogens. Insufficient in vivo studies monitoring sRNA expression attest to the difficulties in collecting such data, we therefore developed a non-mammalian infection model using larval Galleria mellonella to analyze the roles of Staphylococcus aureus sRNAs during larval infection and to quickly determine possible sRNA involvement in staphylococcal virulence before proceeding to more complicated animal testing. We began by using the model to test infected larvae for immunohistochemical evidence of infection as well as host inflammatory responses over time. To monitor sRNA expression during infection, total RNAs were extracted from the larvae and invading bacteria at different time points. The expression profiles of the tested sRNAs were distinct and they fluctuated over time, with expression of both sprD and sprC increased during infection and associated with mortality, while rnalll expression remained barely detectable over time. A strong correlation was observed between sprD expression and the mortality. To confirm these results, we used sRNA-knockout mutants to investigate sRNA involvement in Staphylococcus aureus pathogenesis, finding that the decrease in death rates is delayed when either sprD or sprC was lacking. These results demonstrate the relevance of this G. mellonella model for investigating the role of sRNAs as transcriptional regulators involved in staphylococcal virulence. This insect model provides a fast and easy method for monitoring sRNA (and mRNA) participation in S. aureus pathogenesis, and can also be used for other human bacterial pathogens. 


\section{INTRODUCTION}

The Gram-positive Staphylococcus aureus bacterium is a major human and animal pathogen associated with mortality and morbidity worldwide. In humans, S. aureus is responsible for both community-acquired and healthcare-related infections ranging from superficial to very serious or fatal diseases, including osteomyelitis, bacteremia, and endocarditis (Tong et al., 2015). S. aureus is also a mucosal and skin mucous commensal bacterium, colonizing about $30 \%$ of healthy individuals (van Belkum et al., 2009). The nose is the main ecological niche, and nasal carriage is a key determinant for colonization at other sites (Wertheim et al., 2005), with asymptomatic nasal carriage a major risk factor for subsequent infection (von Eiff et al., 2001).

As both a commensal and a pathogen, S. aureus possesses a large number of factors involved in immunomodulation and/or virulence, including adhesins, toxins, and immunomodulatory proteins that it expresses only when necessary and in a coordinated manner. The bacteria quickly adapt to various environments that change due to stresses from host immune systems or nutrient deprivation, therefore $S$. aureus survives and grows through selective modulation of its metabolism and fitness. This implies a reprogramming of its intricate network of gene regulation, triggering the expression of genes that are essential for survival in the immediate environment, and extinguishing unnecessary ones. In addition to sRNAs, twocomponent systems (TCS) and DNA-binding proteins regulate metabolism and virulence factor expression (Jenul and Horswill, 2018), and the agr quorum-sensing system is the most significant TCS in S. aureus (Novick, 2003). RNAIII is the effector of this agr system (Bronesky et al., 2016) and was also one of the first sRNAs reported in S. aureus (Novick et al., 1993). RNAIII is a multifunctional RNA that codes for $\delta$-hemolysin, and it directly regulates the expression of at least $12 \mathrm{mRNA}$ targets (Raina et al., 2018).

In the SRD Staphylococcal regulatory RNAs database, Sassi et al. listed all sRNAs identified as being expressed by various strains of S. aureus and other Staphylococcaceae (Sassi et al., 2015). The $S$. aureus sRNA targets have only been identified for a few sRNAs, mostly by MS2 tagging (Lalaouna and Massé, 2015). Among these, at least seven are involved in virulence regulation: RNAIII (Srn_3910), SprC (Srn_3610), SprD (Srn_3800), SprX (Srn_3820), RsaA (Srn_1510), Teg49 (Srn_1550), and SSR42 (Srn_4470) (Novick, 2003; Chabelskaya et al., 2010; Morrison et al., 2012; Romilly et al., 2014; Le Pabic et al., 2015; Kathirvel et al., 2016; Manna et al., 2018). Most of these pioneering studies have compared sRNA-deleted and isogenic strains with respect to animal mortality, bacterial load in infected organs, or biofilm formation. Only a few studies have monitored in situ expression of sRNAs (Song et al., 2012; Szafranska et al., 2014), which differ from the levels encountered in growth media. Recent publications on $S$. aureus transcriptional adaptation in vitro and during infection or colonization (Chaffin et al., 2012; Szafranska et al., 2014; Jenkins et al., 2015; Chaves-Moreno et al., 2016; Deng et al., 2019; Ibberson and Whiteley, 2019) have highlighted major differences between in vitro and in vivo transcriptional gene regulation. This suggests that in situ exploration of the regulatory networks involving sRNAs in human pathogens is essential for understanding their roles.

The purpose of this study was to gain further insights into $S$. aureus sRNA functions during infection by assessing their expression levels in situ in infected organisms. To do this, we used a non-mammalian Galleria mellonella model to investigate riboregulations at the transcriptomic level. We provide evidence that the sRNA expression patterns in infected animals differs sharply from the levels in vitro, with a progressive accumulation of the sRNAs SprC and SprD in the larvae up to 4 days after infection. Moreover, SprC and SprD probably act as virulence factors during larval infection, since their presence and expression lead to fewer survivors. This G. mellonella infection model is thus shown to be a reliable tool for investigating the riboregulations involved in bacterial virulence.

\section{MATERIALS AND METHODS}

\section{Strains, Media, and Genetics}

All strains used are listed in Supplementary Table 1. For the development of the G. mellonella infection model, we used the $S$. aureus S75 strain obtained from a bacteremia patient. This strain is a multilocus sequence type 8 (ST8) and has a t190 spa type. The S75 genome was sequenced and deposited in the DDBJ/ EMBL/GeneBank (accession number PRJNA273632). It has $2,728,924$ base pairs, and is close to the NCTC 8325 reference strain, with 2,327 orthologous genes in common. For knock-out experiments, we used strains HG003- $\Delta$ sprC, HG001- $\Delta$ sprX, HG001- $\Delta$ sprD, and HG003-4rnaIII. These were constructed in our lab in HG001 and HG003 backgrounds, and both are derivatives of NCTC 8325 (Herbert et al., 2010). For rnaIII overexpression, we used a QIAprep spin miniprep kit (Quiagen) on the S. aureus Newman strain to extract the vector and transfer it to HG003 to create pRMC3-rnaIII. All strains were grown in Luria-Bertani broth (LB) at $150 \mathrm{rpm}$ at $37^{\circ} \mathrm{C}$, and in the presence of $10 \mu \mathrm{g} / \mathrm{ml}$ chloramphenicol for HG003-pRMC3-rnaIII. For larval infection, overnight cultures were centrifuged at 3,000 rpm for 10 minutes then washed twice with sterile PBS. The inocula were prepared in sterile PBS by OD adjustment, verified by plating on tryptic soy agar after serial dilutions, then incubated for 24 hours at $37^{\circ} \mathrm{C}$.

\section{Galleria mellonella Infection Model}

The G. mellonella larvae were purchased from Sud-Est Appats (Queige, France). They were stored in the dark at $4^{\circ} \mathrm{C}$, and used within 7 days of delivery. To standardize the experiments, we selected larvae weighting about 250-300 mg and without color alteration. They were incubated at room temperature for 2 hours before injection, then disinfected externally with $70 \%$ ethanol. The infection was carried out using a KDS 100 automated syringe pump (KD Scientific) and a $300 \mu$ Hamilton syringe. Haemocoel was injected with $10 \mu \mathrm{l}$ of the S75 strain at $10^{6}, 10^{7}$, $10^{8}$, or $10^{9} \mathrm{CFU} / \mathrm{ml}$. The infected larvae were placed on Petri dishes and incubated at $37^{\circ} \mathrm{C}$. Mortality was monitored daily for 6 days, and larvae were considered dead when they were 
immobile, no longer responding to stimuli, and melanized. For each condition, 3 independent experiments were performed using 10 infected larvae. The controls were uninjected larvae and larvae injected with $10 \mu \mathrm{l}$ of sterile PBS. For the experiments using sRNA-deleted isogenic strains, either HG003 or HG001 was used as the control. For rnaIII overexpression experiments, pRMC3-rnaIII stability was assessed by randomly plating selected colonies grown from fat-body homogenates on agar containing $10 \mu \mathrm{g} / \mathrm{ml}$ of chloramphenicol at different time points after infection.

\section{Bacterial Growth in the Larvae}

One hundred larvae were infected with $10^{6}$ CFU S. aureus S75. At different times post-infection, 3 living larvae were randomly selected, disinfected with $70 \%$ ethanol, dried, and ice-chilled for 10 minutes. The hemolymph and fat bodies were separated from each larva and pooled. Briefly, after an incision with a scalpel, the larvae were squeezed to collect hemolymph, and pooled into a microcentrifuge tube collection. On average, 15 to $40 \mu \mathrm{l}$ of hemolymph per larva were collected. The remaining fat bodies were mechanically homogenized with sterile distilled water at $4^{\circ} \mathrm{C}$. These collections were diluted with sterile distilled water, plated on Baird-Parker agar, and incubated for 48 hours at $37^{\circ} \mathrm{C}$. The bacterial loads were estimated by counting the colonyforming units in each larva at each time point. The in vivo growth curve was monitored every day for 6 days. To investigate early growth, the same experiment was performed but this time just observing the fat bodies over 24 hours, plating every 4 hours. For each condition, 3 independent experiments were performed.

\section{Immunohistochemistry}

We challenged 50 larvae with $10^{6} \mathrm{CFU}$ S75, and 2 living larvae were randomly selected at various times post-infection. Infected and uninfected larvae were fixed for 2 weeks in $4 \%$ neutral buffered formalin. For efficient embedding, the larvae were sectioned longitudinally before being placed into paraffin and processed in a Shandon Excelsior ES (Thermo Scientific). The paraffin-embedded tissues were cut into $4-\mu \mathrm{m}$ sections using a Microm HM340E microtome with section transfer system (Thermo Fisher). These sections were mounted on positively charged SuperFrost Plus slides (VWR) then dried for 60 minutes at $58^{\circ} \mathrm{C}$. Immunohistochemical staining was performed using a Ventana purple-detection kit and their DISCOVERY ULTRA automated stainer. Following dewaxing with EZ prep solution (Ventana) at $75^{\circ} \mathrm{C}$ for 8 minutes, endogenous peroxidase was blocked for 12 minutes at $37^{\circ} \mathrm{C}$ with DISCOVERY Inhibitor (Ventana). After rinsing, the slides were incubated for 60 minutes at $37^{\circ} \mathrm{C}$ with Bio-Rad rabbit polyclonal anti-S. aureus antibodies (0300-0084). Signal enhancement and detection were performed using the DISCOVERY purple kit. Slides were counterstained for 16 minutes with Ventana hematoxylin II (790-2208) and for 4 minutes with Ventana bluing reagent (760-2037). They were then rinsed, manually dehydrated, and cover-slipped. Image acquisition was done with NanoZoomer (Hamamastu Photonics) and analyzed using the accompanying NDP.view2 software.

\section{Bacterial Isolation and RNA Extraction}

The protocol for differential lysis was adapted from Robbe-Saule et al. (2017), Two living larvae were randomly selected, externally disinfected with $70 \%$ ethanol, dried, then placed into Petri dishes. Using sterile forceps holding the head of the larvae, the larval contents were extracted by applying pressure using a $10 \mathrm{ml}$ syringe plunger. This step allowed the cuticle and the larval contents to be correctly separated. After removing their cuticles, larval contents were mechanically homogenized in $5 \mathrm{ml}$ of sterile PBS and centrifuged at 2,500 rpm for 5 minutes. The supernatant was recovered then combined with $50 \mathrm{mM}$ calcium chloride and $1 \mathrm{ml}$ Proteinase $\mathrm{K}$ solution $(12 \mathrm{mg} / \mathrm{ml})$ supplemented with $1 \mathrm{M}$ tris (hydroxylmethyl)aminomethane hydrochloride ( $\mathrm{pH} 8)$. This mix was incubated for 30 minutes at $50^{\circ} \mathrm{C}$, then centrifuged at 13,000 rpm for 20 minutes at $4^{\circ} \mathrm{C}$. For total bacteria RNA extractions, pellets were suspended in $500 \mu \mathrm{l}$ of lysis buffer $(20 \mathrm{mM}$ sodium acetate, $1 \mathrm{mM}$ EDTA, $0.5 \%$ SDS $\mathrm{pH}$ 5.5) and transferred into $1.5 \mathrm{ml}$ RNase-free centrifuge tubes containing $500 \mu \mathrm{l}$ of zirconium beads and $500 \mu \mathrm{l}$ of phenol ( $\mathrm{pH} 4)$. Cells were broken up in a FastPrep FP120 cell disruptor (MP Biomedicals). RNA was extracted by the phenol-chloroform method. The top clear layer was removed and RNA was precipitated overnight at $-80^{\circ} \mathrm{C}$ in isopropanol supplemented with $0.3 \mathrm{M}$ sodium acetate and glycogen. Finally, RNAs were pelleted by centrifugation at 13,000 rpm for 30 minutes at $4^{\circ} \mathrm{C}$. The pellets were then cleaned with $70 \%$ ethanol, dried, and dissolved in RNase-free water. To eliminate DNA contamination, samples were processed with the TURBO DNA-free kit (Thermo Fisher) as per the manufacturer's recommendations. A second precipitation was performed to remove putative contamination, with RNAs treated with $100 \%$ ethanol supplemented with $0.3 \mathrm{M}$ sodium acetate and glycogen. RNA quality and quantity were assessed using a NanoDrop spectrophotometer (Thermo Fisher) and agarose gel electrophoresis.

We used proteinase $\mathrm{K}$ to create a differential lysis method that would preferentially degrade larval tissue, and we verified its efficiency through method development. Briefly, we first controlled that proteinase $\mathrm{K}$ did not significantly damage bacterial cells by comparing numbers of CFU of $S$. aureus before and after incubation with proteinase $\mathrm{K}$ in vitro. Then, we applied this method to RNA extraction, and we assessed the integrity of RNAs. Finally, we tested increasing concentrations of proteinase $\mathrm{K}$ on infected larvae.

Bacterial RNA extraction was also performed from in vitro cultures with a Proteinase $\mathrm{K}$ solution at both the midexponential and stationary phases in LB broth. At designated time in vitro bacteria cultures were incubated for 30 minutes at $50^{\circ} \mathrm{C}$ with a proteinase $\mathrm{K}$ solution, then centrifuged at 13,000 rpm for 10 minutes at $4^{\circ} \mathrm{C}$. From the pellet, total RNA was extracted according to the protocol described above.

\section{Monitoring RNA Expression Levels}

We challenged 100 larvae with $10^{6} \mathrm{CFU}$ S75, and quantitative reverse transcription PCR (qRT-PCR) was used to monitor sRNA expression during larval infection. We analyzed RNAIII, SprC, SprD, SprX, and RsaA, sRNAs present and expressed in the S75 strain (Bordeau et al., 2016), as well as the mRNA 
controls GyrB and SigA. All primers used are listed in Supplementary Table 2. RNA samples were reversetranscribed and amplified using a high-capacity cDNA reverse transcription kit and the PowerUp SYBR green master mix (Thermo Fisher) as recommended by the manufacturer. Reverse transcription was performed using $0.3 \mu \mathrm{g}$ of RNA in $10 \mu \mathrm{l}$ of nuclease free water, and $10 \mu \mathrm{l}$ of the $2 \mathrm{X}$ RT master mix. The reverse transcription run followed the manufacturer's recommendations. Resulting cDNA were stored at $-20^{\circ} \mathrm{C}$ until qPCR. For qPCR, the reaction volume was $20 \mu \mathrm{l}$ comprising $10 \mu \mathrm{l}$ SYBR Green 10X master mix, $5 \mu \mathrm{l}$ cDNA diluted 1/100, forward and reverse primers at a final concentration of $500 \mathrm{nM}$, completed with nuclease free water. The reaction was performed on the Applied Biosystems 7500 Real-Time PCR System using the standard mode.

Two different methods were used for data interpretation: comparison against the in vitro RNA expression levels of S75 at the exponential and stationary growth phases; and comparison of sRNA transcript levels in the larvae at an early (12-hour) postinfection step against the levels at various post-infection times. sRNA expression was normalized against housekeeping mRNA transcripts, and their relative expression levels were inferred using the $\Delta \Delta \mathrm{Ct}$ method. All experiments were performed in triplicate.

\section{Statistical Analysis}

G. mellonella survival profiles were determined using the logrank test and Kaplan-Meier survival plots. For daily mortality comparisons, a Student's t-test was used to compare the expressions in sRNA-deleted and isogenic strains. Sample $t$ tests were used for comparing the sRNA profiles between experiments, for instance in vivo versus in vitro expression during the exponential or stationary growth phases. To monitor the trend in larval sRNA expressions at each selected post-infection time point, we analyzed the variance and did multiple comparisons of means using Tukey contrasts. A twosample test was used to analyze sRNA expression in the larvae during the first 24 hours after infection, then up to 96 hours later. Larval sRNA expression and risk-adjusted mortality rates were compared using Spearman's correlation coefficient. The riskadjusted mortality corresponded to the ratio of the number of dead larvae to the number of larvae still alive at the time of the experiment. Results were considered statistically significant when $p<0.05$.

\section{RESULTS}

\section{Setting up a G. mellonella Infection Model}

To determine larval susceptibility to infection with S. aureus S75, we injected them with different inocula and monitored mortality daily. We observed that survival rates in G. mellonella were dependent on the S75 dosage (Figure 1A). No deaths or melanization were detected either in the control group or after injecting $10^{4} \mathrm{CFU}$ of S75 (Figure 1B). However, all larvae died within 48 hours after injection with $10^{7} \mathrm{CFU}$. At day 6, mortality was $40 \%$ and $80 \%$ after injection with $10^{5}$ or $10^{6} \mathrm{CFU}$, respectively. Dead larvae were immobile as well as black due to melanization (Figure 1C). For our model, we selected a dose of $10^{6} \mathrm{CFU}$, as a gradual reduction in larval survival rates occurred over the 6-day experiments with that amount.

To determine S75 growth within the larvae, they were infected by $10^{6} \mathrm{CFU}$, then 3 were sacrificed at different times postinfection to count the $S$. aureus bacteria present in the hemolymph and fat bodies. In the fat body, bacterial growth was biphasic, with a fast increase from $10^{6}$ to $10^{9} \mathrm{CFU} /$ larva up to 24 hours post-infection, and a steady load of about $10^{9} \mathrm{CFU} /$ larva from day 1 to day 6 post-infection (Figure 1D). Growth was different in the hemolymph, with bacterial loads increasing up to 72 hours but not exceeding $6.10^{6} \mathrm{CFU} /$ larva (Supplementary Figure 1).

\section{Monitoring S. aureus Infection of Larvae With Immunohistochemical Staining}

We used immunohistochemical staining and analysis to monitor infection with $S$. aureus S75 as well as its impact on various larval tissues up to 4 days after infection (Figure 2). In the control larvae, the hemocyte immune cells were subcuticular, scattered in the fat body and around the digestive tract (Figure 2A). We observed an immune response in the infected larvae as early as 1 day post-infection, with hemocyte recruitment around the bacteria, which are clustered into 'grape-like' shapes (Figure 2B). Bacteria often co-localize with host immune-cells and form nodules with each other (Figure 2C). Infection foci spread progressively, affecting the entire larval body, including its nervous system (Supplementary Figure 2). During infection, the immune response increased, with a predominance of pigmented nodules reflecting melanization (Figure 2D). Nodules were composed of bacteria, melanin, as well as numerous hemocytes, and we could visualize morphologically distinct immune cells (Figures 2E, F).

\section{Monitoring S. aureus sRNA Expression During Infection}

The challenge was to extract enough intact bacterial RNA for qRT-PCR assays of their expression levels. To create a differential lysis method which would only degrade larval tissues, we used Proteinase $\mathrm{K}$. We began by showing that this serine protease does not damage bacterial cells, since the $S$. aureus CFU counts were not statistically different with or without Proteinase $\mathrm{K}$ (Supplementary Figure 3A). We also saw that Proteinase $\mathrm{K}$ did not influence the quality of the bacterial RNA (Supplementary Figure 3B). We were thus able to extract intact bacterial RNA from the mixture of eukaryotic and prokaryotic cells, and as expected, we observed an increased ratio of bacterial to larval RNA (Supplementary Figure 3C). Therefore, increasing concentrations of Proteinase K improved detection of $S$. aureus targets, and this was shown by the lowered cycle thresholds for the housekeeping genes gyrB and sigA (Supplementary Table 3). Finally, we extracted bacterial RNA from the whole infected larvae, at time points between 12 and 96 hours after infection. 
A

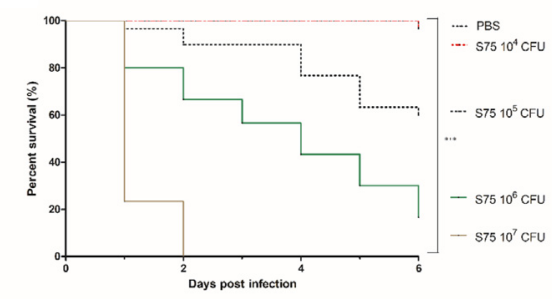

C

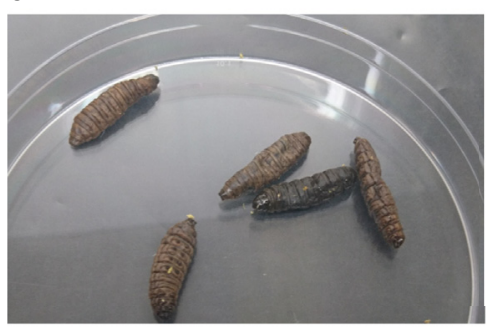

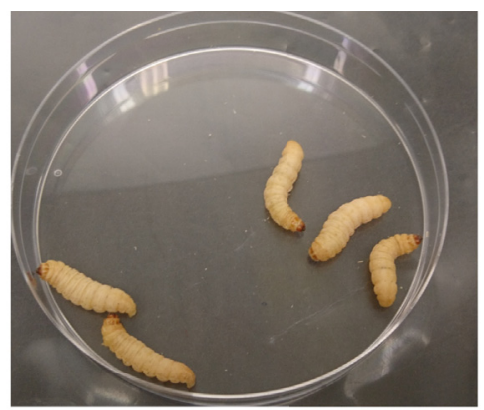

D

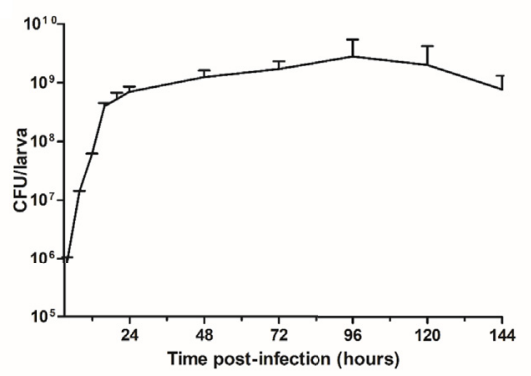

FIGURE 1 | Kaplan-Meier survival plots and bacterial levels in infected Galleria mellonella larvae infected with Staphylococcus aureus. (A) Survival of G. mellonella larvae after inoculation with $10^{4}$ to $10^{7} \mathrm{CFU}$ of the $\mathrm{S}$. aureus S75 strain. Plots show an average of 3 independent experiments with 10 larvae per group ( $\mathrm{N}=150$ ), with mortality monitored daily for 6 days. PBS-injected larvae were the negative control. Significant differences were defined as ${ }^{\star \star \star} p<0.001$. (B) $G$. mellonella larvae on day 6 after being challenged with $10 \mu$ of PBS. (C) Dead G. mellonella larvae at day 6 after infection with $10^{6}$ CFU of S. aureus S75. (D) Time evolution of bacterial burden in homogenized cuticle-free larvae infected by $10^{6} \mathrm{CFU}$ of $\mathrm{S}$. aureus S75. Data are the sum of 3 independent experiments, with 100 larvae per experiment $(\mathrm{N}=300)$. Error bars represent the standard deviations $(\mathrm{SD})$.

We assessed $S$. aureus sRNA transcript levels in situ at various times post-infection and compared these to those seen at the exponential and stationary levels in culture media (Figures 3A, B). We found that rnaIII and $r s a A$ expression levels were relatively stable across the 4-day infection of the larvae. Interestingly, those of $s p r C, s p r D$, and $s p r X$ progressively increased during infection, but the expression levels of these sRNAs in the larvae were dependent upon the in vitro 'comparator' set and the phase examined. Indeed, ratios ranged from 2-6 when stationary phase cultures were used as a control, and from 10-40 when selecting the exponential phase cultures. In addition, whereas the in vivo gene expressions of $\operatorname{sprD}, \operatorname{sprC}$, and $\operatorname{spr} X$ were higher than both control conditions (respectively $p<0.0001,0.0001$, and 0.001 when compared with the in vitro exponential phase, and $p=$ $0.0005,0.003$ and 0.001 when compared with the stationary phase), rsaA was more expressed in vivo than in the in vitro exponential phase $(p=0.001)$, but less expressed in vivo than the in vitro stationary phase $(p=0.0001)$. Such differences highlight the difficulty of choosing a good in vitro comparator. It is noteworthy that no significant difference was observed with rnaIII.

Since it was difficult to compare the in vitro and in vivo sRNA expression levels, we compared in vivo sRNA expression to the first qRT-PCR value obtained at the beginning of infection, 12 hours after injection (Figure 3C). We saw a trend for $\operatorname{sprC}$ and $s p r D$, which were both upregulated and had peaks 96 hour after injection. While $\operatorname{sprD}$ was significantly upregulated 16-96 hours post-infection $(p=0.043)$, the increase observed for $\operatorname{spr} C$ was not statistically significant. $s p r X$ was increased early, with a peak 48 hours after infection, and its expression was significantly higher at that point than at 16 hours $(p=0.029)$. rnaIII was weakly expressed at all-time points, and none of the observed variations were significant. No significant variations were observed for $r s a A$.

sRNA expression levels were examined in the periods before (16-20 hours) and after (48-96 hours) the 24-hour post-infection point (Figure 3D). Higher levels of $s p r C, s p r D$, and $s p r X$ were found after the first day of infection $(p=0.0001, p<0.0001$, and $p=0.0007$, respectively), while rnaIII and $r s a A$ expression levels stayed relatively even.

\section{sRNAs Are Involved in G. mellonella Virulence}

Larvae mortality started 1 day after infection, when the bacterial load had reached about $10^{9}$ bacteria per larva, and death increased continuously thereafter (Figure 4A). Since time postinfection and mortality are linked, to minimize interpretation bias, we looked at how sRNA levels corresponded to the riskadjusted mortality. In fact, $s p r D$ expression levels are strongly correlated to the risk-adjusted mortality $(r=0.87, p<0.0001)$, implying that the more SprD is expressed, the more virulent the strain (Figure 4B). 


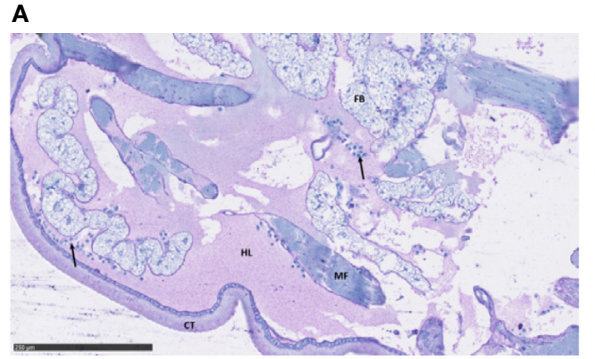

C

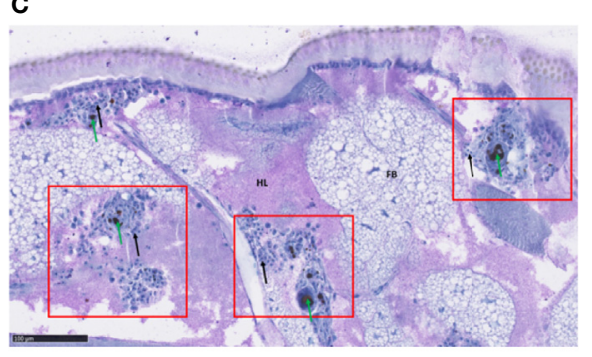

E

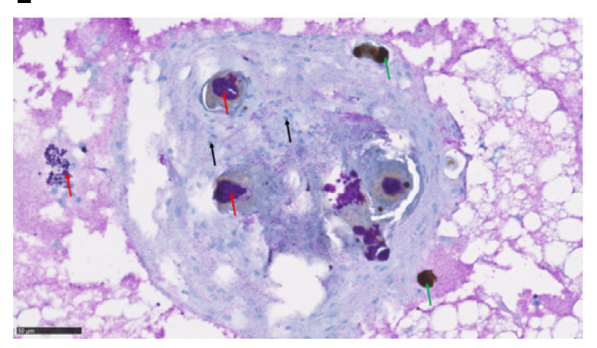

B

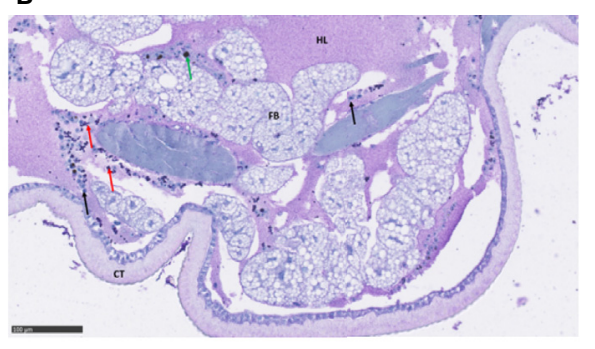

D

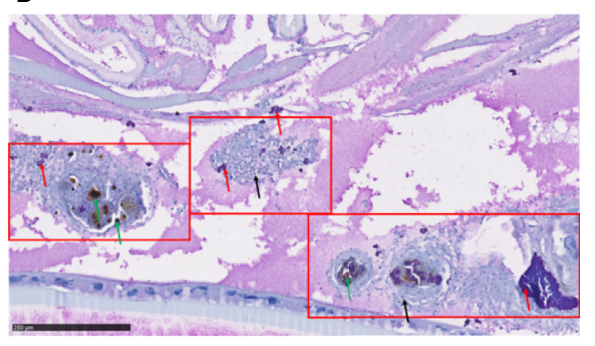

$\mathbf{F}$

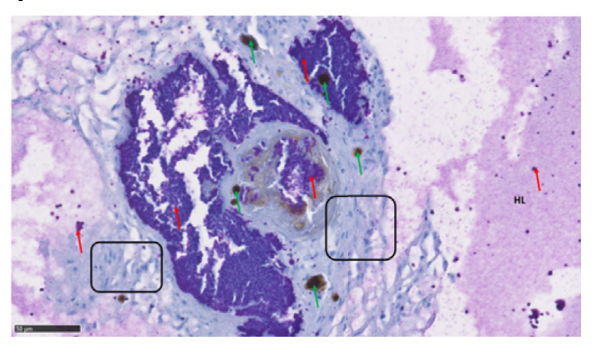

FIGURE 2 | Immunohistochemical analysis of Galleria mellonella larva sections. Immunohistochemical analysis and hematoxylin staining were performed to visualize bacteria within the infected host and to examine histological sections. G. mellonella larvae were challenged with $10^{6} \mathrm{CFU}$ S. aureus S75, then collected at fixed times and examined. Shown are: (A) non-infected larvae; (B, C) infected larvae 24 hours after infection, showing evidence of immune activation; (D) infected larvae at 72 hours, with numerous nodules in the presence of hemocytes and bacteria; and (E, F) higher-magnification views of pigmented nodules at 96 hours after infection. $S$. aureus bacteria appeared in purple and are indicated with red arrows, hemocytes were stained in blue, with a deep blue nucleus and are indicated by black arrows, melanization (black spots) by green arrows, nodule clusters are outlined in red squares, and hemocytes of different morphology framed in black boxes. Scale bars are indicated on each picture. Key: CT, cuticle; HL, hemolymph; FB, fat body; MF, muscle fibers.

To look for connections between sRNA expression and virulence, we compared the mortality induced by sRNAdeletion strains to that of isogenic controls. We examined S75 and two another strains, HG001 or HG003, both of which are derived from the NCTC 8325 reference strain that is phylogenetically related to S75. We did not observe any differences in larval mortality in these three ST8 strains (Supplementary Figure 4).

At all tested times, larval mortality was significantly reduced $(p=0.03)$ in comparison with the control after infection with HG003- $\Delta \operatorname{sprC}$ (Figure 5A). However, only late mortality (at day $6)$, was significantly reduced $(p=0.01)$ in HG001- $\Delta$ sprD infection as compared to the isogenic strain (Figure 5B). In contrast, no significant differences $(p=0.70$ and $p=0.64$, respectively) were seen after infection with HG001- $\Delta$ sprX or HG003-ArnaIII (Figures 5C, D).

Since rnaIII expression in the larvae was very low, we wondered whether overexpressing rnaIII would affect virulence. We found that rnaIII overexpression significantly increased mortality $(p=0.007)$ (Figure 5E), with enhanced larval necrosis and a complete destruction of larval tissue (Supplementary Figure 5). High rnaIII expression thus seems to be detrimental during larval infection.

\section{DISCUSSION}

Here, we present a G. mellonella model set up to study the impacts of $S$. aureus riboregulations during infection. Indeed, accumulating evidence has shown the importance of numerous sRNAs in bacterial pathogenicity (Novick, 2003; Chabelskaya et al., 2010; Morrison et al., 2012; Romilly et al., 2014; Le Pabic et al., 2015; Kathirvel et al., 2016; Manna et al., 2018). The larvae of this greater wax moth have been extensively used to study the virulence of many microorganisms (Tsai et al., 2016) and for testing the efficacy of antimicrobial compounds (Cutuli et al., 2019) 
A

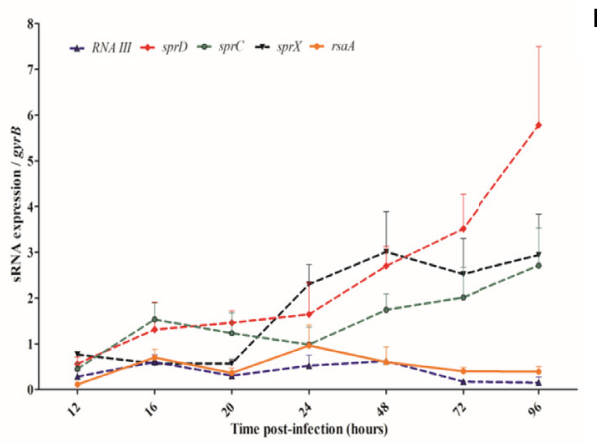

C

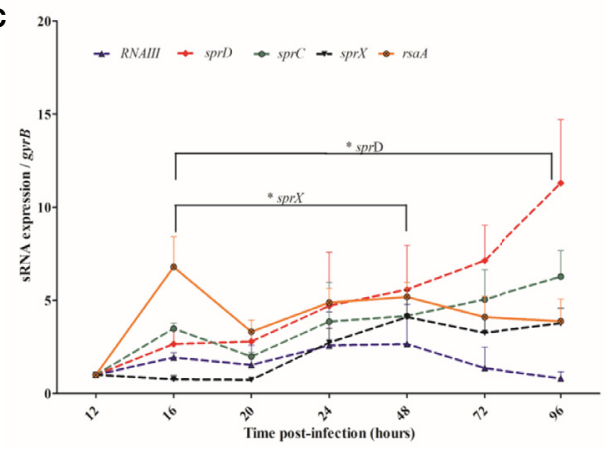

$B$

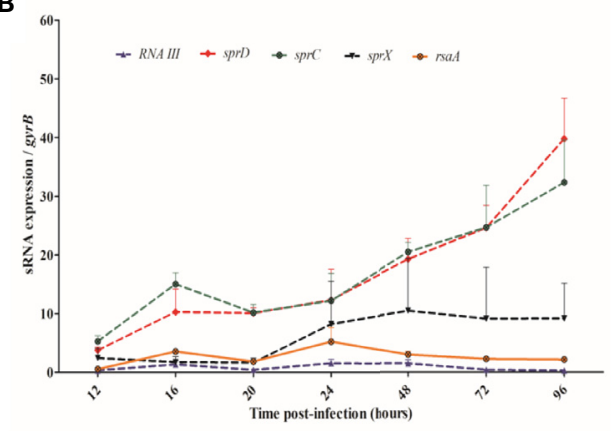

D

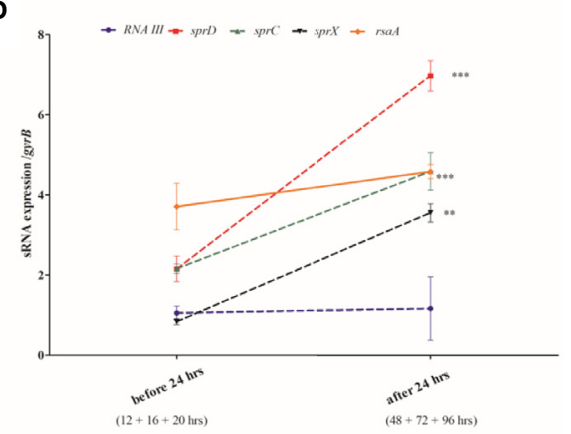

FIGURE 3 | Monitoring sRNA expression from 12 to 96 hours after Staphylococcus aureus infection of larva (A, B) sRNA expression levels in the Galleria mellonella larvae compared with the levels found at the stationary (A) and exponential (B) growth phases in liquid media. (C) sRNA expression levels in the larvae at various time points are compared to their values 12 hours after infection. (D) Comparison of pooled sRNA expression in the larvae before and after the 24-hour postinfection point. sRNA expression monitored by qRT-PCR was normalized against GyrB mRNA, and 3 independent experiments were realized for each condition, with 100 larvae per experiment $(\mathrm{N}=300)$. Error bars represent the SDs. Significant differences were defined as ${ }^{\star} p<0.05 ;{ }^{\star \star} p<0.01 ;$ and ${ }^{* \star *} p<0.001$.

for several reasons: costs are low; no specific training or equipment is required; and many larvae can be infected simultaneously. In addition, invertebrates do not possess nociceptors and are thus not sensitive to pain, so there are no restrictive ethical rules as exist for vertebrate use (Bismuth et al., 2019). G. mellonella lacks adaptive immunity, but the organism develops an innate immune response that is similar to that of vertebrates, including cellular and humoral responses which are responsible for nodulation and phagocytosis (Pereira et al., 2018). Unlike Caenorhabditis elegans and Drosophila melanogaster, infections can be performed at $37^{\circ} \mathrm{C}$ in $\mathrm{G}$. mellonella, which is an advantage for studying the bacterial virulence of S. aureus or other human pathogens. G. mellonella infections can be caused either via ingestion or by intrahemocoelic injection, and the latter method allows for close control of the inoculum (Ramarao et al., 2012). In most studies, the model has been used to examine the antimicrobial activity of drugs or to screen for S. aureus virulence factors (Quiblier et al., 2013; Ferro et al., 2016; Silva et al., 2017). A previous work showed that G. mellonella could also be used for other purposes, especially for examining $S$. aureus interaction with the larval immune response and for how the bacteria induces the expression of immune-related peptides (Sheehan et al., 2019). We therefore used this model to investigate riboregulations in staphylococcal virulence. As proof of concept, we applied it to examine sRNAs already established as having major impacts on virulence.

Consistent with a recent study (Sheehan et al., 2019), we saw an early bacterial multiplication that triggered an immune response in the form of hemocyte recruitment. Majority of hemocytes are phagocytic cells similar to vertebrate neutrophils which are involved in abscess formation (Kobayashi et al., 2015). During S. aureus infection, we visualized nodules which could correspond to abscesses in vertebrate tissues. The number of nodules increased during infection as well as being spread throughout the entire larva, implying systemic infection. During human infection, circulating bacteria are scarce (Opota et al., 2015), as they are rapidly killed by phagocytes. The surviving bacteria migrate, accumulating in host organs and causing tissue damages and/or abscesses. Similarly, in infected larvae, the bacterial load is higher than in the fat body compared to the hemolymph which is the larval equivalent to the bloodstream.

We investigated how staphylococcal sRNAs were expressed during infection. The 'proof of concept' was achieved after we examined a subset of five sRNAs: RsaA and RNAIII which are both expressed from the core genome; and SprC, SprD, and SprX from pathogenicity islands. Indeed, with the exception of 
A

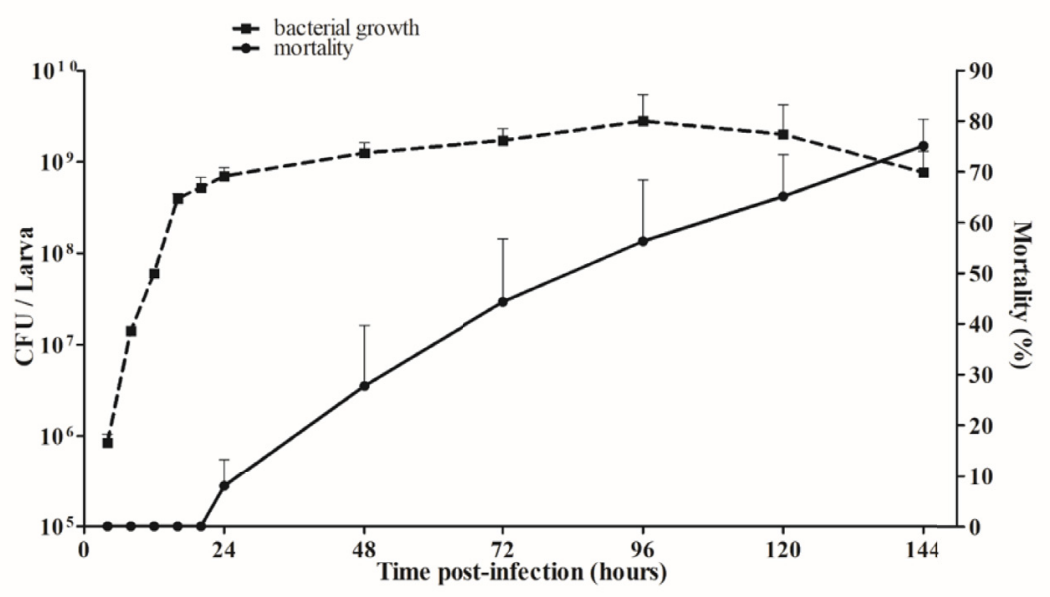

B

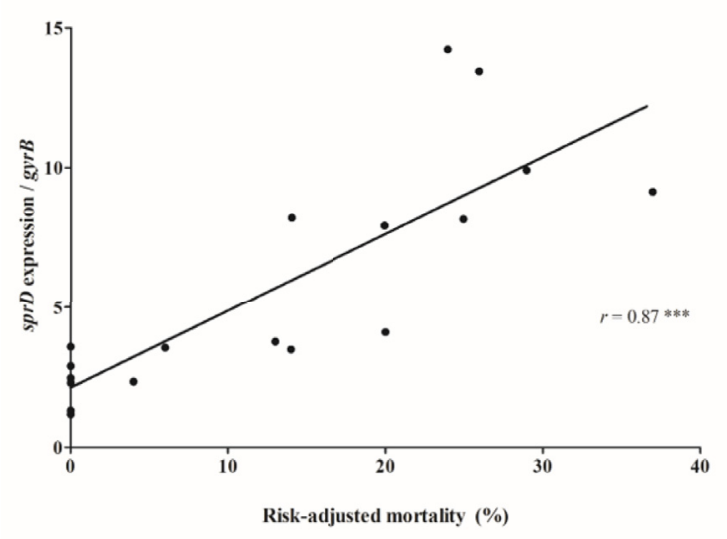

FIGURE 4 | Relationship between mortality and SRNA expression levels. (A) Correlation between growth of Staphylococcus aureus (CFU/larva) and the mortality of infected Galleria mellonella larvae. (B) Correlation between sprD expression and risk-adjusted mortality. The in vivo transcript levels of sprD were calculated to those for gyrB mRNA and compared to the expression obtained 12 hours after infection. Each point represents relative sprD expression during infection associated with the risk-adjusted mortality at the same time point, and corresponds to the sum of 3 independent experiments, with 100 larvae per experiment ( $N=300)$. Key: $r$, Sperman's coefficient; ${ }^{* *} p<0.001$.

RNAIII, the expression levels of each of these sRNAs in the larvae differed from what can be observed in liquid cultures during the exponential or stationary growth phases. Therefore, in vitro sRNA expression does not mimic that of in vivo conditions, a difference already observed for other staphylococcal sRNAs isolated from nasal carriers, abscesses, cystic fibrosis patients, and mouse-model bone infections (Song et al., 2012; Szafranska et al., 2014). Frequently, the in vivo expression of these sRNAs has been arbitrarily compared to in vitro exponential or stationary growth phases, and it is therefore difficult to determine the precise evolution of staphylococcal sRNAs during disease (Song et al., 2012; Szafranska et al., 2014). Our observations agree with this, proving the importance of the calibrator used for analyzing the results. To compare bacterial colonization at different stages of infection, the in vivo gene expression of $S$. aureus was previously monitored (without an in vitro calibrator) in mice and cotton rats presenting with nasal colonization, bacteremia, or heart lesions (Jenkins et al., 2015).
We therefore decided to apply this method to the examination of in vivo sRNA expression. In our model, we measured sRNA expression 12 to 96 hours after infection, setting the initial 12hour value as our reference. We found that the infection stage influences sRNA expression. Variations in the expression levels of $\operatorname{sprD}$ and $s p r X$, as well as their accumulation in bacterial cells throughout the infection, suggest that these sRNAs both contribute to infection. It also indicates that sRNAs are not expressed continuously, and that they are tightly regulated at the different stages of infection.

Larval mortality began at 24 hours after infection, and increased progressively. There is no link between bacterial growth and mortality, as the bacterial load remained stable between days 1 and 6. In vitro, the agr quorum-sensing (QS) system senses population density, promoting the expression of secreted proteins and inhibiting adhesins via RNAIII regulation (Novick, 2003). This system does not seem to be activated during larval infection, since the post-infection expression of rnaIII 
A

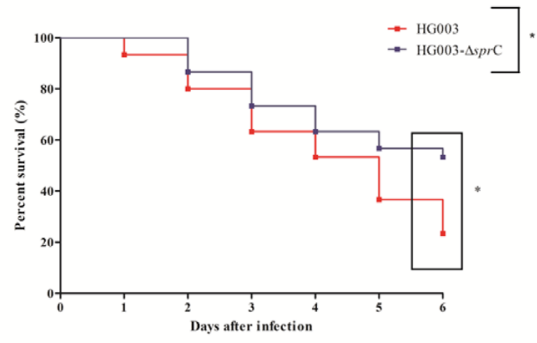

C

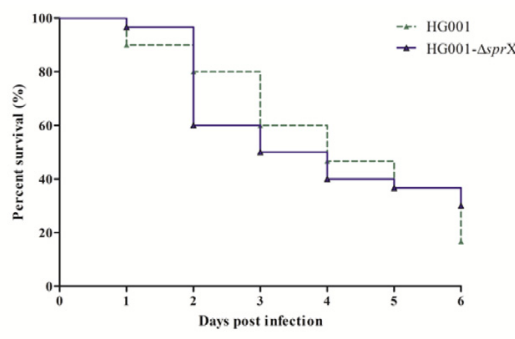

E

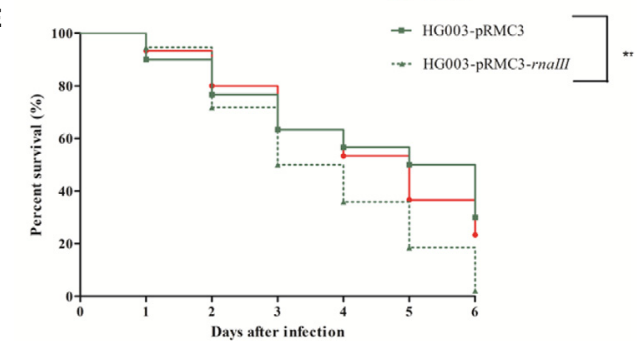

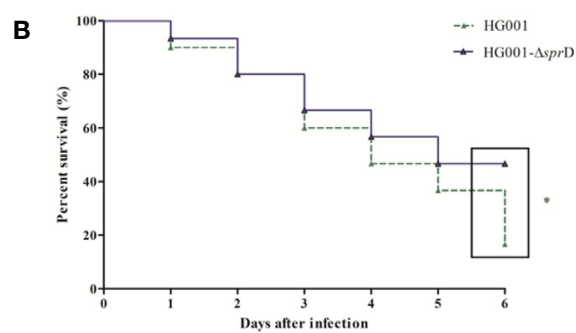

D

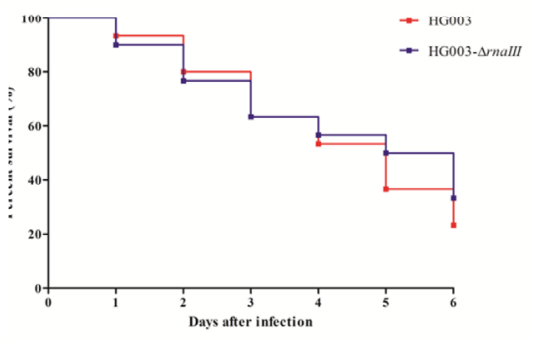

FIGURE 5 | Kaplan-Meier survival plots of Galleria mellonella larvae infected with various forms of Staphyllococcus aureus. Larvae were injected with $10^{6}$ CFU of wild-type HG001 or HG003, three types of isogenic sRNA mutants, or with a strain overexpressing rnalll. PBS-injected larvae were used as negative controls. Shown are the survival rates over 6 days for the following: (A) HG003 and its HG003- $\Delta$ sprC isogenic mutant; (B) HG001 and HG001- $\Delta$ sprD; (C) HG001 and HG001- $\Delta$ sprX; (D) HG003 and HG003- $\Delta$ rnalll; and (E) HG003 compared to both HG003-pRMC3 and HG003-pRMC3-rnalll. Significant differences were defined as * $p$ < 0.05; ${ }^{\star \star} p<0.01$. Data are shown from 3 independent experiments using 10 larvae per group for each condition.

remains low. These results were further supported by the lack of significant differences we observed in larval mortality between HG003 and the HG003- $\Delta$ rnaIII mutant. RNAIII does not affect S. aureus survival on fruit flies (Garcia-Lara et al., 2005), suggesting that RNAIII might not play a key role in virulence in invertebrates. However, we showed that increasing rnaIII expression actually promotes mortality, with efficient larval necrosis. Furthermore, we found low rnaIII expression levels in the infected larvae, just as low amounts were detected in a mouse model of osteomyelitis, in human abscesses, during murine vaginal colonization, and in the lungs of cystic fibrosis patients (Song et al., 2012; Szafranska et al., 2014; Deng et al., 2019; Ibberson et al., 2019). Nevertheless, this sRNA is still involved in virulence since disruption of the agr QS system inhibits the upregulation of many toxins and proteases and a $\triangle a g r B$ strain protects mice from mortality (Date et al., 2014). A $\Delta$ rnaIII strain was also shown to attenuate virulence in a murine intracranial abscess model (Gong et al., 2014). Numerous studies have reported agr downregulation in multiple human host niches responding to different environmental cues (Dastgheyb and
Otto, 2015). Indeed, environmental cues modify the behavior of $S$. aureus and counter its agr system, which is sensitive to many stresses or host-derived factors such as the reactive oxygen species that are produced in infected larvae by activated hemocytes (Tsai et al., 2016; Jenul and Horswill, 2018). This could prevent agr activation, explaining the low expression of rnaIII, and thereby counterbalance $S$. aureus virulence. In contrast, rnaIII overexpression probably overwhelms the host immune response, which would explain the resulting virulence enhancement. Further studies are needed to clarify the central role of RNAIII in virulence regulation.

Staphylococcal virulence is also affected by others sRNAs: SprC (Le Pabic et al., 2015), SprD (Chabelskaya et al., 2010), and SprX (Kathirvel et al., 2016). The expression levels of $\operatorname{sprC}$ and $\operatorname{sprD}$ are increased when the bacteria are in contact with human serum (Carroll et al., 2016). However, little monitoring of their expression levels during animal infection has been done, so this was one objective of our study. For both SprC and SprD, expression increased progressively in infected larvae, and we observed decreased larval mortality with the $\Delta s p r C$ strain. Since 
SprC was previously shown to attenuate staphylococcal virulence in a mouse infection model (Le Pabic et al., 2015), our data are somewhat surprising, indicating that results might vary depending on the animal model and strains used. Results of Le Pabic et al. were obtained using a Newman strain which among other changes differs with a mutation in saeS (Herbert et al., 2010). This mutation induces a particular exoprotein profile by comparison with HG003. Similarly, the survival profile in a sepsis mouse model was not strictly superposable between these two strains (Herbert et al., 2010). These differences may explain these inconsistent results. A strong positive correlation between $\operatorname{sprD}$ expression and risk-adjusted mortality was also observed. These preliminary results need to be confirmed in a mammalian model and in humans. S. aureus bacteremia is a leading cause of morbidity and mortality (Yilmaz et al., 2016), yet not enough relevant biomarkers have been identified for reliable prediction of clinical outcomes. The monitoring of $s p r D$ expression alone could be a potential biomarker of severity, and our observations have confirmed that SprD plays a major role in bacterial virulence in two different animal models of infection (Chabelskaya et al., 2010). SprD has one identified target, Sbi, which is an immune-evasion molecule acting at the interface between the innate and adaptive immune systems (Smith et al., 2011), and which is downregulated by SprD (Chabelskaya et al., 2010). There is no specific adaptive system in the greater wax moth, nevertheless, the staphylococcal Sbi was shown to be important in the Galleria model since the mortality was attenuated with a $\Delta s b i$ USA300 strain (Zhao et al., 2020). So it is likely that SprD acts on other molecular targets to influence staphylococcal virulence.

Our study confirms the importance of sRNAs in S. aureus infection in animals, and the handy Galleria mellonella infection model can easily be extended to the investigation of all 50 bona fide sRNAs known to be expressed by S. aureus (Liu et al., 2018). sRNAs belong to complex gene regulatory networks (Fechter et al., 2014), and testing their involvement in virulence requires an understanding of their connections and interactions within the entire regulon. Our next step will be to perform a transcriptomic analysis to compare sRNA-deletion and isogenic strains in order to obtain a more comprehensive view of their participation in virulence.

\section{DATA AVAILABILITY STATEMENT}

The datasets presented in this study can be found in online repositories. The names of the repository/repositories and accession number(s) can be found in the article/ Supplementary Material.

\section{AUTHOR CONTRIBUTIONS}

Conceptualization: GM and P-YD. Experiments: GM and AR. Microscopy: GG and GM. Statistics: ME and GM. Writing original draft: GM. Writing - review and editing: GM, AR, BF, and P-YD. All authors contributed to the article and approved the submitted version.

\section{ACKNOWLEDGMENTS}

We are most grateful to Yoann Augagneur and Svetlana Chabelskaya for providing $S$. aureus mutant strains and to Marc Hallier for the pRMC3 vector. We also thank Mohamed Sassi for phylogenetic data and annotation of the S75 strain genome. Thanks to Alain Fautrel for his help in exploring microscopy techniques and Juliana Berland for critical reading of this manuscript.

\section{SUPPLEMENTARY MATERIAL}

The Supplementary Material for this article can be found online at: https://www.frontiersin.org/articles/10.3389/fcimb.2021. 631710/full\#supplementary-material

Supplementary Figure 1 | Post-infection bacterial burden in G. mellonella. Graph showing S. aureus S75 bacterial burden over time in the hemolymph of larvae infected by $10^{6} \mathrm{CFU}$. Data are the result of 3 independent experiments, each using 100 larvae per group. Error bars represent the SDs.

Supplementary Figure 2 | Cross-sectional analysis for monitoring infection in the larvae. (A) PBS-injected larvae, the negative controls. (B) Larvae were injected with $10^{6} \mathrm{CFU}$ of S. aureus S75, and are shown here 96 hours later. Each black ellipse represents a nodule showing melanization, and the nodules are scattered around the entire larvae, including within the nervous system (NS).

Supplementary Figure 3 | Effect of Proteinase K on total RNA extractions. (A) Bacteria in the stationary phase were treated with $3 \mathrm{mg} / \mathrm{ml}$ Proteinase $\mathrm{K}$ (proK) or PBS for 30 minutes at $50^{\circ} \mathrm{C}$. Results are the means of 3 independent experiments and are expressed in CFU/ml. (B) Total bacterial RNA counts after agarose gel electrophoresis and ethidium bromide exposure with (+) or without (-) a $3 \mathrm{mg} / \mathrm{ml}$ Proteinase $\mathrm{K}$ solution. Bacterial RNA was extracted during the stationary phase after overnight culture. (C) Total RNA extraction of G. mellonella infected with $10^{8} \mathrm{CFU}$ of S. aureus S75. For each condition, 5 larvae were infected, and 2 were randomly chosen for RNA extraction $(\mathrm{N}=6)$. RNA extraction was performed 30 minutes after injection, and several concentrations of Proteinase $\mathrm{K}$ were applied. For each condition, after DNAse process, total RNA quality and quantity were assessed using a NanoDrop spectrophotometer and 0.5\% agarose gel electrophoresis.

Supplementary Figure 4 | Comparison of Kaplan-Meier survival plots of larvae infected with ST8 strains. Survival of G. mellonella larvae infected with $10^{6} \mathrm{CFU}$ of the $S$. aureus strains S75, HG003, and HG001. Mortality was monitored for 6 days. The plot is the result of 3 independent experiments using 10 larvae per group, and PBS-injected larvae were used as a negative control.

Supplementary Figure 5 | RNAll overexpression and macroscopic effects on infected G. mellonella larvae. Larvae were inoculated with $10^{6} \mathrm{CFU}$ and mortality monitored for 6 days, at which point dead larvae were observed. (A) Dead and melanized larvae after HG003-pRMC3 infection. (B) Larval necrosis was increased after infection with HG003-pRMC3-rnalll.

Supplementary Table 1 | Strains and vectors used for G. mellonella infection.

Supplementary Table 2 | Primers used for qRTPCR.

Supplementary Table 3 | Proteinase K Effects on the cycle thresholds of $S$. aureus housekeeping genes observed in qRTPCR. 


\section{REFERENCES}

Bismuth, H., Aussel, L., and Ezraty, B. (2019). La teigne Galleria mellonella pour les études hôte-pathogène. Med. Sci. (Paris) 35, 346-351. doi: 10.1051/medsci/2019071

Bordeau, V., Cady, A., Revest, M., Rostan, O., Sassi, M., Tattevin, P., et al. (2016). Staphylococcus aureus Regulatory RNAs as Potential Biomarkers for Bloodstream Infections. Emerg. Infect. Dis. 22, 1570-1578. doi: 10.3201/ eid2209.151801

Bronesky, D., Wu, Z., Marzi, S., Walter, P., Geissmann, T., Moreau, K., et al. (2016). Staphylococcus aureus RNAIII and Its Regulon Link Quorum Sensing, Stress Responses, Metabolic Adaptation, and Regulation of Virulence Gene Expression. Annu. Rev. Microbiol. 70, 299-316. doi: 10.1146/annurev-micro102215-095708

Carroll, R. K., Weiss, A., Broach, W. H., Wiemels, R. E., Mogen, A. B., Rice, K. C., et al. (2016). Genome-wide Annotation, Identification, and Global Transcriptomic Analysis of Regulatory or Small RNA Gene Expression in Staphylococcus aureus. mBio 7, e01990-15. doi: 10.1128/mBio.01990-15

Chabelskaya, S., Gaillot, O., and Felden, B. (2010). A Staphylococcus aureus small RNA is required for bacterial virulence and regulates the expression of an immune-evasion molecule. PloS Pathog. 6, e1000927. doi: 10.1371/ journal.ppat.1000927

Chaffin, D. O., Taylor, D., Skerrett, S. J., and Rubens, C. E. (2012). Changes in the Staphylococcus aureus transcriptome during early adaptation to the lung. PloS One 7, e41329. doi: 10.1371/journal.pone.0041329

Chaves-Moreno, D., Wos-Oxley, M. L., Jáuregui, R., Medina, E., Oxley, A. P., and Pieper, D. H. (2016). Exploring the transcriptome of Staphylococcus aureus in its natural niche. Sci. Rep. 6, 33174. doi: 10.1038/srep33174

Cutuli, M. A., Petronio Petronio, G., Vergalito, F., Magnifico, I., Pietrangelo, L., Venditti, N., et al. (2019). Galleria mellonella as a consolidated in vivo model hosts: New developments in antibacterial strategies and novel drug testing. Virulence 10, 527-541. doi: 10.1080/21505594.2019.1621649

Dastgheyb, S. S., and Otto, M. (2015). Staphylococcal adaptation to diverse physiologic niches: an overview of transcriptomic and phenotypic changes in different biological environments. Future Microbiol. 10, 1981-1995. doi: $10.2217 / \mathrm{fmb} .15 .116$

Date, S. V., Modrusan, Z., Lawrence, M., Morisaki, J. H., Toy, K., Shah, I. M., et al. (2014). Global gene expression of methicillin-resistant Staphylococcus aureus USA300 during human and mouse infection. J. Infect. Dis. 209, 1542-1550. doi: $10.1093 /$ infdis/jit668

Deng, L., Schilcher, K., Burcham, L. R., Kwiecinski, J. M., Johnson, P. M., Head, S. R., et al. (2019). Identification of Key Determinants of Staphylococcus aureus Vaginal Colonization. mBio 10, e02321-19. doi: 10.1128/mBio.02321-19

Fechter, P., Caldelari, I., Lioliou, E., and Romby, P. (2014). Novel aspects of RNA regulation in Staphylococcus aureus. FEBS Lett. 588, 2523-2529. doi: 10.1016/ j.febslet.2014.05.037

Ferro, T. A. F., Araújo, J. M. M., dos Santos Pinto, B. L., dos Santos, J. S., Souza, E. B., da Silva, B. L. R., et al. (2016). Cinnamaldehyde Inhibits Staphylococcus aureus Virulence Factors and Protects against Infection in a Galleria mellonella Model. Front. Microbiol. 7, 2052. doi: 10.3389/fmicb.2016.02052

García-Lara, J., Needham, A. J., and Foster, S. J. (2005). Invertebrates as animal models for Staphylococcus aureus pathogenesis: a window into host-pathogen interaction. FEMS Immunol. Med. Microbiol. 43, 311-323. doi: 10.1016/ j.femsim.2004.11.003

Gong, J., Li, D., Yan, J., Liu, Y., Li, D., Dong, J., et al. (2014). The accessory gene regulator (agr) controls Staphylococcus aureus virulence in a murine intracranial abscesses model. Braz. J. Infect. Dis. 18, 501-506. doi: 10.1016/ j.bjid.2014.03.005

Herbert, S., Ziebandt, A.-K., Ohlsen, K., Schäfer, T., Hecker, M., Albrecht, D., et al. (2010). Repair of Global Regulators in Staphylococcus aureus 8325 and Comparative Analysis with Other Clinical Isolates. Infect. Immun. 78, 2877. doi: 10.1128/IAI.00088-10

Ibberson, C. B., and Whiteley, M. (2019). The Staphylococcus aureus Transcriptome during Cystic Fibrosis Lung Infection. mBio 10, de02774-19. doi: $10.1128 / \mathrm{mBio} .02774-19$

Jenkins, A., Diep, B. A., Mai, T. T., Vo, N. H., Warrener, P., Suzich, J., et al. (2015). Differential Expression and Roles of Staphylococcus aureus Virulence Determinants during Colonization and Disease. mBio 6, e02272-14. doi: $10.1128 / \mathrm{mBio} .02272-14$
Jenul, C., and Horswill, A. R. (2018). Regulation of Staphylococcus aureus virulence. Microbiol. Spectr. 6, 669-686. doi: 10.1128/microbiolspec.GPP3-0031-2018

Kathirvel, M., Buchad, H., and Nair, M. (2016). Enhancement of the pathogenicity of Staphylococcus aureus strain Newman by a small noncoding RNA SprX1. Med. Microbiol. Immunol. 205, 563-574. doi: 10.1007/s00430-016-0467-9

Kobayashi, S. D., Malachowa, N., and DeLeo, F. R. (2015). Pathogenesis of Staphylococcus aureus Abscesses. Am. J. Pathol. 185, 1518-1527. doi: 10.1016/j.ajpath.2014.11.030

Lalaouna, D., and Massé, E. (2015). Identification of sRNA interacting with a transcript of interest using MS2-affinity purification coupled with RNA sequencing (MAPS) technology. Genom. Data 5, 136-138. doi: 10.1016/j.gdata.2015.05.033

Le Pabic, H., Germain-Amiot, N., Bordeau, V., and Felden, B. (2015). A bacterial regulatory RNA attenuates virulence, spread and human host cell phagocytosis. Nucleic Acids Res. 43, 9232-9248. doi: 10.1093/nar/gkv783

Liu, W., Rochat, T., Toffano-Nioche, C., Le Lam, T. N., Bouloc, P., and Morvan, C. (2018). Assessment of Bona Fide sRNAs in Staphylococcus aureus. Front. Microbiol. 9, 228. doi: 10.3389/fmicb.2018.00228

Manna, A. C., Kim, S., Cengher, L., Corvaglia, A., Leo, S., Francois, P., et al. (2018). Small RNA teg49 Is Derived from a sarA Transcript and Regulates Virulence Genes Independent of SarA in Staphylococcus aureus. Infect. Immun. 86, e00635-17. doi: 10.1128/IAI.00635-17

Morrison, J. M., Miller, E. W., Benson, M. A., Alonzo, F., Yoong, P., Torres, V. J., et al. (2012). Characterization of SSR42, a Novel Virulence Factor Regulatory RNA That Contributes to the Pathogenesis of a Staphylococcus aureus USA300 Representative. J. Bacteriol. 194, 2924-2938. doi: 10.1128/JB.06708-11

Novick, R. P., Ross, H. F., Projan, S. J., Kornblum, J., Kreiswirth, B., and Moghazeh, S. (1993). Synthesis of staphylococcal virulence factors is controlled by a regulatory RNA molecule. ЕMBO J. 12, 3967-3975. doi: 10.1002/j.1460-2075.1993.tb06074.x

Novick, R. P. (2003). Autoinduction and signal transduction in the regulation of staphylococcal virulence. Mol. Microbiol. 48, 1429-1449. doi: 10.1046/j.13652958.2003.03526.x

Pereira, T. C., de Barros, P. P., Fugisaki, L. R. de O., Rossoni, R. D., Ribeiro, F. de. C., de Menezes, R. T., et al. (2018). Recent Advances in the Use of Galleria mellonella Model to Study Immune Responses against Human Pathogens. J. Fungi 4, 128. doi: $10.3390 /$ jof 4040128

Opota, O., Jaton, K., and Greub, G. (2015). Microbial diagnosis of bloodstream infection: toward molecular diagnosis directly from blood. Clin. Microbiol. Infect. 21, 323-331. doi: 10.1016/j.cmi.2015.02.005

Quiblier, C., Seidl, K., Roschitzki, B., Zinkernagel, A. S., Berger-Bächi, B., and Senn, M. M. (2013). Secretome Analysis Defines the Major Role of SecDF in Staphylococcus aureus Virulence. PloS One 8, e63513. doi: 10.1371/journal.pone.0063513

Raina, M., King, A., Bianco, C., and Vanderpool, C. K. (2018). Dual-function RNAs. Microbiol. Spectr. 6, 5. doi: 10.1128/microbiolspec.RWR-0032-2018

Ramarao, N., Nielsen-Leroux, C., and Lereclus, D. (2012). The Insect Galleria mellonella as a Powerful Infection Model to Investigate Bacterial Pathogenesis. J. Vis. Exp. e4392. doi: 10.3791/4392

Robbe-Saule, M., Babonneau, J., Sismeiro, O., Marsollier, L., and Marion, E. (2017). An Optimized Method for Extracting Bacterial RNA from Mouse Skin Tissue Colonized by Mycobacterium ulcerans. Front. Microbiol. 8, 512. doi: $10.3389 /$ fmicb.2017.00512

Romilly, C., Lays, C., Tomasini, A., Caldelari, I., Benito, Y., Hammann, P., et al. (2014). A Non-Coding RNA Promotes Bacterial Persistence and Decreases Virulence by Regulating a Regulator in Staphylococcus aureus. PloS Pathog. 10, e1003979. doi: 10.1371/journal.ppat.1003979

Sassi, M., Augagneur, Y., Mauro, T., Ivain, L., Chabelskaya, S., Hallier, M., et al. (2015). SRD: a Staphylococcus regulatory RNA database. RNA 21, 1005-1017. doi: $10.1261 /$ rna.049346.114

Sheehan, G., Dixon, A., and Kavanagh, K. (2019). Utilization of Galleria mellonella larvae to characterize the development of Staphylococcus aureus infection. Microbiology 165, 863-875. doi: 10.1099/mic.0.000813

Silva, L. N., Da Hora, G. C. A., Soares, T. A., Bojer, M. S., Ingmer, H., Macedo, A. J., et al. (2017). Myricetin protects Galleria mellonella against Staphylococcus aureus infection and inhibits multiple virulence factors. Sci. Rep. 7, 2823. doi: 10.1038/s41598-017-02712-1

Smith, E. J., Visai, L., Kerrigan, S. W., Speziale, P., and Foster, T. J. (2011). The Sbi Protein Is a Multifunctional Immune Evasion Factor of Staphylococcus aureus. Infect. Immun. 79, 3801-3809. doi: 10.1128/IAI.05075-11 
Song, J., Lays, C., Vandenesch, F., Benito, Y., Bes, M., Chu, Y., et al. (2012). The Expression of Small Regulatory RNAs in Clinical Samples Reflects the Different Life Styles of Staphylococcus aureus in Colonization vs. Infection. PloS One 7, e37294. doi: 10.1371/journal.pone.0037294

Szafranska, A. K., Oxley, A. P. A., Chaves-Moreno, D., Horst, S. A., Roßlenbroich, S., Peters, G., et al. (2014). High-resolution transcriptomic analysis of the adaptive response of Staphylococcus aureus during acute and chronic phases of osteomyelitis. mBio 5, e01775-14. doi: 10.1128/mBio.01775-14

Tong, S. Y. C., Davis, J. S., Eichenberger, E., Holland, T. L., and Fowler, V. G. (2015). Staphylococcus aureus infections: epidemiology, pathophysiology, clinical manifestations, and management. Clin. Microbiol. Rev. 28, 603-661. doi: 10.1128/CMR.00134-14

Tsai, C. J.-Y., Loh, J. M. S., and Proft, T. (2016). Galleria mellonella infection models for the study of bacterial diseases and for antimicrobial drug testing. Virulence 7, 214-229. doi: 10.1080/21505594.2015.1135289

van Belkum, A., Verkaik, N. J., de Vogel, C. P., Boelens, H. A., Verveer, J., Nouwen, J. L., et al. (2009). Reclassification of Staphylococcus aureus nasal carriage types. J. Infect. Dis. 199, 1820-1826. doi: 10.1086/599119

von Eiff, C., Becker, K., Machka, K., Stammer, H., and Peters, G. (2001). Nasal carriage as a source of Staphylococcus aureus bacteremia. Study Group. N. Engl. J. Med. 344, 11-16. doi: 10.1056/NEJM200101043440102

Wertheim, H. F. L., Melles, D. C., Vos, M. C., van Leeuwen, W., van Belkum, A., Verbrugh, H. A., et al. (2005). The role of nasal carriage in Staphylococcus aureus infections. Lancet Infect. Dis. 5, 751-762. doi: 10.1016/S1473-3099(05) 70295-4

Yilmaz, M., Elaldi, N., Balkan, İ. İ, Arslan, F., Batirel, A. A., Bakici, M. Z., et al. (2016). Mortality predictors of Staphylococcus aureus bacteremia: a prospective multicenter study. Ann. Clin. Microbiol. Antimicrob. 15, 7. doi: 10.1186/s12941-016-0122-8

Zhao, X., Chlebowicz-Flissikowska, M. A., Wang, M., Murguia, E. V., Jong, A., Becher, D., et al. (2020). Exoproteomic profiling uncovers critical determinants for virulence of livestock-associated and human-originated Staphylococcus aureus ST398 strains. Virulence 11, 947-963. doi: 10.1080/ 21505594.2020.1793525

Conflict of Interest: The authors declare that the research was conducted in the absence of any commercial or financial relationships that could be constructed as a potential conflict of interest.

Copyright (C) 2021 Ménard, Rouillon, Ghukasyan, Emily, Felden and Donnio. This is an open-access article distributed under the terms of the Creative Commons Attribution License (CC BY). The use, distribution or reproduction in other forums is permitted, provided the original author(s) and the copyright owner(s) are credited and that the original publication in this journal is cited, in accordance with accepted academic practice. No use, distribution or reproduction is permitted which does not comply with these terms. 
OPEN ACCESS

Edited by:

Gheyath Khaled Nasrallah,

Qatar University, Qatar

Reviewed by:

Chang-Shi Chen,

National Cheng Kung University,

Taiwan

Antoni Hendrickx,

National Institute for Public Health and

the Environment, Netherlands

*Correspondence:

Natalia Kirienko

kirienko@rice.edu

Specialty section: This article was submitted to

Bacteria and Host,

a section of the journal

Frontiers in Cellular and

Infection Microbiology

Received: 12 February 2021

Accepted: 13 April 2021

Published: 29 April 2021

Citation:

Revtovich AV, Tjahjono E,

Singh KV, Hanson BM,

Murray BE and Kirienko NV (2021) Development and Characterization

of High-Throughput Caenorhabditis

elegans - Enterococcus faecium

Infection Model.

Front. Cell. Infect. Microbiol. 11:667327.

doi: 10.3389/fcimb.2021.667327

\section{Development and Characterization of High-Throughput Caenorhabditis elegans - Enterococcus faecium Infection Model}

\author{
Alexey V. Revtovich ${ }^{1}$, Elissa Tjahjono ${ }^{1}$, Kavindra V. Singh ${ }^{2}$, Blake M. Hanson ${ }^{2,3}$, \\ Barbara E. Murray ${ }^{2,4}$ and Natalia V. Kirienko ${ }^{1 *}$ \\ ${ }^{1}$ Department of BioSciences, Rice University, Houston, TX, United States, ${ }^{2}$ Division of Infectious Diseases, McGovern \\ Medical School, University of Texas Health Science Center, Houston, TX. United States, ${ }^{3}$ Center for Infectious Diseases, \\ School of Public Health, University of Texas Health Science Center, Houston, TX, United States, ${ }^{4}$ Department of \\ Microbiology and Molecular Genetics, McGovern Medical School, University of Texas Health Science Center, Houston, \\ TX, United States
}

The genus Enterococcus includes two Gram-positive pathogens of particular clinical relevance: E. faecalis and E. faecium. Infections with each of these pathogens are becoming more frequent, particularly in the case of hospital-acquired infections. Like most other bacterial species of clinical importance, antimicrobial resistance (and, specifically, multidrug resistance) is an increasing threat, with both species considered to be of particular importance by the World Health Organization and the US Centers for Disease Control. The threat of antimicrobial resistance is exacerbated by the staggering difference in the speeds of development for the discovery and development of the antimicrobials versus resistance mechanisms. In the search for alternative strategies, modulation of host-pathogen interactions in general, and virulence inhibition in particular, have drawn substantial attention. Unfortunately, these approaches require a fairly comprehensive understanding of virulence determinants. This requirement is complicated by the fact that enterococcal infection models generally require vertebrates, making them slow, expensive, and ethically problematic, particularly when considering the thousands of animals that would be needed for the early stages of experimentation. To address this problem, we developed the first high-throughput C. elegans-E. faecium infection model involving host death. Importantly, this model recapitulates many key aspects of murine peritonitis models, including utilizing similar virulence determinants. Additionally, host death is independent of peroxide production, unlike other $E$. faecium-C. elegans virulence models, which allows the assessment of other virulence factors. Using this system, we analyzed a panel of lab strains with deletions of targeted virulence factors. Although removal of certain virulence factors (e.g., $\Delta f m s 15)$ was sufficient to affect virulence, multiple deletions were generally required to affect pathogenesis, suggesting that host-pathogen interactions are multifactorial. These data were corroborated 
by genomic analysis of selected isolates with high and low levels of virulence. We anticipate that this platform will be useful for identifying new treatments for $E$. faecium infection.

Keywords: C. elegans, E. faecium, high-throughput screen, virulence, host-pathogen interaction

\section{INTRODUCTION}

Enterococci, particularly Enterococcus faecalis and E. faecium, are categorized as serious threats by the Centers for Disease Control and Prevention and since the 1970s have risen to be some of the most commonly isolated Gram-positive organisms responsible for nosocomial infections. In 2017 alone, there were approximately 55,000 infections with enterococcal species in the United states, resulting in 5,400 deaths (CDC, 2019). Infections commonly take the form of endocarditis, urinary tract infections (UTI), peritonitis, or meningitis, amongst others (Goh et al., 2017). Healthcare costs to treat enterococcal infection reached $\$ 540$ million (CDC, 2019). Frequently, infections are acquired in healthcare settings, leading to endangerment of immunocompromised patients, including those who are infected with SARS-CoV-2 (Ramos-Martinez et al., 2020; Kampmeier et al., 2020; Bonazzetti et al., 2021). Enterococci are increasingly developing resistance to multiple antimicrobial drugs and disinfectants, including vancomycin, the drug of last resort (Kafil and Asgharzadeh, 2014; Levitus et al., 2020). In fact, about $30 \%$ of healthcare-associated enterococcal infections are resistant to vancomycin (CDC, 2019) and vancomycin-resistant enterococci (VRE) (the vast majority of which are E. faecium) often develop resistance to other antibiotics, leading to the requirement for intensive and invasive treatments. Resistance prolongs hospitalization and increases the likelihood for patients to contract secondary infections.

E. faecalis and E. faecium, both of which are commonly found as intestinal commensals (Huycke et al., 1998), are the two most commonly isolated enterococcal species in clinical samples. Although E. faecalis historically was better known as a human pathogen, E. faecium has risen to comprise over one-third of cases (Iwen et al., 1997; Treitman et al., 2005; Goh et al., 2017). This rise has led to suggestions that it may have recently acquired new virulence determinants (Kafil and Asgharzadeh, 2014; Davis et al., 2020) in addition to its frequent resistance to vancomycin, ampicillin, and other antimicrobials (Murray, 2000; Mundy et al., 2000).

Although it has been studied for several decades, relatively little is known about the molecular mechanisms involved in infection and pathogenesis in E. faecium (Freitas et al., 2018; Gao et al., 2018). Like most Gram-positive bacteria, it possesses a polysaccharide capsule and teichoic acid derivatives in its cell wall. It also includes several secreted factors, a secreted adhesion factor known as SagA, and a broad array of other attachment factors, including the $e b p A B C_{f m}$ pilus operon (Hendrickx et al., 2008; Sillanpää et al., 2010; Montealegre et al., 2016), collagen adhesins ( $\mathrm{acm}, \mathrm{scm})$ (Nallapareddy et al., 2006; Nallapareddy et al., 2008; Sillanpää et al., 2008), and surface proteins (fms, esp) (Willems et al., 2001; Hendrickx et al., 2007; Sillanpää et al., 2008). Most of these virulence factors have been demonstrated to contribute to E. faecium virulence in murine endocarditis and/or
UTI models. However, it is quite likely that many other virulence determinants remain undiscovered.

Unfortunately, the model organisms available to study $E$. faecium virulence are predominantly mammals. Although effective, these models are strongly constrained in terms of throughput, are expensive, and are fraught with ethical concerns. This makes them inappropriate for large scale screens to identify novel virulence factor or therapeutic compounds (Garsin et al., 2014; Goh et al., 2017). In contrast, Caenorhabditis elegans infection models are cheap, fast, and share a surprising amount of infection biology with human beings. A C. elegans - Enterococcus pathosystem had previously been developed (Garsin et al., 2001), however only $E$. faecalis was able to cause persistent infection in $C$. elegans gut and kill adult worms. Unfortunately, although C. elegans has generally proven susceptible to infection with E. faecalis, it has been difficult to develop an infection system that is lethal, uses relevant pathogenic determinants, and is amenable to highthroughput techniques for E. faecium. For example, an agar-based system was developed that involved anaerobic growth of E. faecium (Moy et al., 2004), but killing depended upon hydrogen peroxide production. The prolific capacity of mammalian cells to produce catalase makes this virulence determinant less relevant in human infection. Another study indicated that $C$. elegans could be killed with E. faecium provided that one of several innate immune pathways were compromised (Yuen and Ausubel, 2018), but the virulence determinants involved were not identified and using worms with signaling defects may result in spurious outcomes.

In this study, we developed a liquid-based C. elegans-E. faecium pathogenesis assay. This assay was based on our conventional $P$. aeruginosa Liquid Killing assay (Kirienko et al., 2013; Anderson et al., 2018), which was modified for Enterococcus growth and to automatically quantify death, enabling unbiased and high-throughput studies. Using this assay, we tested available E. faecium lab strains containing mutations in several known virulence factors. We found that, although C. elegans death was not correlated with bacterial growth, highly virulent E. faecium strains possessed greater host colonization ability. We also tested the pathogenesis of $\sim 120$ E. faecium isolates, originating from hospital, commensal, microbiota, and animals, and observed that none of the clades had dramatically increased or reduced virulence. This marks an important step towards understanding the pathogenicity of $E$. faecium and will aid in the search of new therapeutic procedures.

\section{MATERIALS AND METHODS}

\section{Strains}

C. elegans strains SS104 [glp-4(bn2)], N2 wild type, and pmk-1 ( $k m 25)$ were maintained on standard nematode growth medium 
(NGM) (Stiernagle, 2006) seeded with Escherichia coli strain OP50 as a food source at $15^{\circ} \mathrm{C}$, unless otherwise noted (Stiernagle, 2006). C. elegans - E. faecium experiments were performed at $25^{\circ} \mathrm{C}$ to induce sterility of temperature-sensitive bn2 allele, similarly to other $g l p-4(b n 2)$-based assays (Moy et al., 2009; Kirienko et al., 2014; Kim et al., 2020). For experiment with $\mathrm{N} 2$ and $p m k-1(\mathrm{~km} 25)$, sterility was induced by feeding worms with $c d c-25.1(R N A i)$-expressing E. coli. Absence of progeny facilitates analysis of assay outcomes.

E. faecium strains used in this study were collected over the past 30 years. The goal of our study was to compare the virulence of E. faecium belonging to different clades in C. elegans model. Therefore the strains representing clinical strains (mostly clade A1), commensal strains from healthy volunteers (mostly clade B), and isolates from animal sources or published clade A2 strains were selected. These isolates included 75 health carederived isolates (mostly clade A1) from USA and Colombia (Arias et al., 2009; Sillanpää et al., 2009; Tran et al., 2013; Diaz et al., 2014), 21 human commensal strains (mostly clade B) (Sillanpää et al., 2009), and 21 isolates derived from animals, animal feed or obtained from published clade A2 strains studies (Sillanpää et al., 2009; Lebreton et al., 2013). Source information for these isolates is provided in Table S1. Genotype of strains with deletions is in Table $\mathbf{1}$ below.

\section{E. faecium Gene Deletions}

Gene deletion mutants of previously described cell wall anchored (CWA) surface proteins with Ig-like folds (Sillanpää et al., 2008) were given $\mathrm{fms}$ designations and were generated in two clinical $E$. faecium strains (TX0082 and TX2154) using previously published methods (Panesso et al., 2011; Somarajan et al., 2014). Deletions included fms18 (ecbA of E. faecium microbial surface component recognizing adhesive matrix molecules (MSCRAMM) (Sillanpää et al., 2008; Hendrickx et al., 2009); fms11 (predicted pilusassociated) (Sillanpää et al., 2008) and scm (second collagen adhesin of E. faecium) (Sillanpää et al., 2008). Two substitutions (Y176A and F192A) were introduced in $\mathrm{acm}$, a previously described MSCRAMM gene (Nallapareddy et al., 2003; Nallapareddy et al., 2008) using published methods (Liu et al., 2007). These strains were designated as TX6111 (E1162 acm $\left.{ }^{\mathrm{Y} 176 \mathrm{~A}, \mathrm{~F} 192 \mathrm{~A}} \Delta \mathrm{fms} 18 \Delta \mathrm{scm}\right)$,

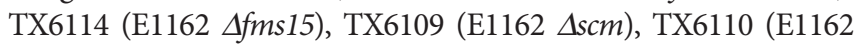
$\mathrm{acm}^{\mathrm{Y} 176 \mathrm{~A}, \mathrm{~F} 192 \mathrm{~A}} \Delta \mathrm{scm}$ ), TX6094 (TX0082 acm ${ }^{\mathrm{Y} 176 \mathrm{~A}, \mathrm{~F} 192 \mathrm{~A}} \Delta \mathrm{fms} 11$ $\Delta s c m$ ), and TX 6097 (TX0082 $\Delta f m s 11 \Delta s c m$ ) and have been listed in strain list in Table $\mathbf{1}$.

\section{C. elegans - Enterococcus Pathogenesis Assays}

For the high-throughput, liquid-based pathogenesis assay, 25 synchronized young adult worms were sorted into 384-well plate. E. faecium strains were grown in deep-well plates at $700 \mathrm{rpm}$ for 16 h. S Basal medium was mixed with brain-heart infusion (BHI) medium (10\%) and Enterococcus faecalis or E. faecium (final OD600: 0.03) were then added into each well. Plates were incubated at $25^{\circ} \mathrm{C}$ until assay completion. At various time points, bacterial OD was measured with a Cytation5 (BioTek Instruments) plate reader and then plates were washed three times. Sytox Orange nucleic acid stain was added into each well and incubated for $12-16 \mathrm{~h}$ to stain dead worms. Plates were then washed three times, worms were transferred into a new 384-well plate, washed three more times, and imaged with Cytation5 automated microscope. Dead worms were quantified with CellProfiler software, similar to previously established pipelines (Conery et al., 2014).

For treatment with catalase, catalase $(7.5 \mathrm{mg} / \mathrm{mL})$ was supplemented in S Basal, BHI, Enterococcus mixture that was then distributed into each well of the 384-well plates.

TABLE 1 | List of $E$. faecium mutants used for this study.

\begin{tabular}{|c|c|c|}
\hline Strain & Description & Reference \\
\hline TX1330 & WT & (Coque et al., 1995) \\
\hline TX6067 & TX1330 Ag/s33-glsB::erm Agls20-glsB1, EryR & (Choudhury et al., 2011) \\
\hline TX2518 & WT & (Kim et al., 2010) \\
\hline TX6089 & TX2158 $\Delta f m s 21-20$ & This study \\
\hline TX2154 & E1162, WT & (Heikens et al., 2007) \\
\hline TX6109 & $\mathrm{E} 1162 \Delta \mathrm{scm}$ & This study \\
\hline TX6110 & $\mathrm{E} 1162 \mathrm{acm}^{\mathrm{Y} 176 \mathrm{~A}, \mathrm{~F} 192 \mathrm{~A}} \Delta \mathrm{scm}$ & This study \\
\hline TX0082 & WT & (Nallapareddy et al., 2006) \\
\hline TX6115 & TX0082 $\Delta w \times / C$ & (Galloway-Peña et al., 2015) \\
\hline TX6094 & TX0082 acm ${ }^{\text {Y176A,F192A }} \Delta f m s 11 \Delta s c m$ & This study \\
\hline TX6108 & TX0082 $\Delta w / c B$ & \\
\hline TX6135 & TX0082 $\Delta W \times I A$ & \\
\hline TX6097 & TX0082 $\Delta f m s 11 \Delta \mathrm{scm}$ & This study \\
\hline
\end{tabular}

Wild-type strains are indicated in bold. 
For killing on agar, 50 young adult worms were transferred onto Enterococcus-BHI plates and incubated at $25^{\circ} \mathrm{C}$. Worms were scored every day for survival; dead worms were removed from assay plates.

\section{Murine Peritonitis Model}

E. faecium strains, TX0016 (clade A) and a commensal isolate, TX1330 (clade B) were tested following our previously published method (Singh et al., 1998). In brief, mice were injected intraperitoneally with appropriate dilutions of bacteria grown in BHI broth, premixed with sterile rat fecal extract (SRFE) and were observed for 5 days for survival. Data (h) were used to compare animal survival/mortality using six mice per group. Comparison of the survival curves was performed using a logrank test with GraphPad Prism 4 for Windows ${ }^{\circledR}$. A $p<0.05$ was considered significant. All experiments were approved by the Animal Welfare committee, University of Texas Health Science Center at Houston.

\section{Colony Forming Unit (CFU) Assay}

75 worms from liquid-based assay were collected into microcentrifuge tubes. Worms were washed three times to remove residual bacteria. After the final wash excess media was aspirated down to $100 \mu \mathrm{L}$. Equal volume of carbide beads was then added into the tubes and vortexed vigorously for $1 \mathrm{~min}$ to break worms. Supernatant was serially diluted and plated on BHI plates.

\section{Genomic Analysis}

Genomic analysis was performed in the usegalaxy.org platform. FASTQ files generated from NGS were submitted for SNPs analysis to snippy (Galaxy version 4.5.0), a bioinformatic tools for rapid bacterial variant calling. Meanwhile, FASTA files were processed with prokka (Galaxy version 1.14.5) for genome annotation, roary (Galaxy version 3.13.0) for genomic alignment, and $R A x M L$ (Galaxy version 1.0.0) to construct a maximum-likelihood phylogenetic tree. Figure 8A was generated with $\mathrm{R}$ packages treeio (version 1.14.3) and ggtree (version 2.4.1).

\section{Microscopy}

Upon 48 h of infection with E. faecium E007 in the liquid-based assay, worms were washed three times to remove residual bacteria, incubated for one hour with or without $20 \mu \mathrm{g} / \mathrm{mL}$ gentamicin, washed three times, and stained with $40 \mu \mathrm{M}$ acridine orange for 4 hours. Worms were then washed three more times to remove unbound dye. Worms were imaged using a Zeiss ApoTome.2 Imager.M2 fluorescent microscope (Carl Zeiss, Germany) with a 20x objective magnification.

\section{RNA Interference Protocol}

RNAi-expressing E. coli HT115 were cultured and seeded onto NGM plates supplemented with $25 \mu \mathrm{g} / \mathrm{mL}$ carbenicillin and 1 mM IPTG. 8,000 synchronized L1 larvae were transferred onto RNAi plates and grown at $25^{\circ} \mathrm{C}$ for 64 hours prior to exposure to pathogens.
RNAi experiments in this study were conducted by using RNAi-competent HT115 obtained from the Ahringer RNAi library (Kamath et al., 2003) and were sequenced prior to use.

\section{Statistical Analysis}

With the exception of the mouse infection model, all experiments were performed in at least three independent biological replicates. The number of worms used per biological replicates is listed in the corresponding legends.

Student's $t$-test analysis was performed to calculate the $p$-values when comparing two groups in an experimental setting. $p$-values were indicated in graphs as follows: NS not significant, ${ }^{*} p<0.05$, ${ }^{* *} p<0.01$, and ${ }^{* * *} p<0.001$.

Figure 2 was generated with $\mathrm{R}$ package ggplot2 (version 3.3.3). Correlation coefficient and $p$-value between $C$. elegans survival and E. faecium OD600 were calculated and indicated on the figure.

Figure 5 was generated with $\mathrm{R}$ package ggplot2 (version 3.3.3). K-means clustering was performed with the 'kmeans' function in R. p-values between Clade A1 and Clade B were indicated on graphs.

\section{RESULTS}

\section{E. faecium E007 Is Pathogenic to C. elegans}

To study E. faecium virulence determinants, we developed the first high-throughput infection model for E. faecium that uses C. elegans as a host. In brief (see Figure 1A), synchronized, young adult $C$. elegans were sorted into 384-well plates. E. faecium or E. faecalis grown on $\mathrm{BHI}$ agar plates were then diluted into $10 \% \mathrm{BHI}$ medium and added to worms at predetermined concentrations. Worms were infected at $25^{\circ} \mathrm{C}$ until assay completion and then were washed several times before staining with a cell-impermeant stain to quantify death. The entire assay can be performed within $\sim 1$ week, and $\sim 90$ different strains can be tested in parallel on a single 384-well plate. Unlike in previously published agar-based systems where only E. faecalis killed the worms (Garsin et al., 2001), our liquid assay showed killing within a week by both $E$. faecalis and $E$. faecium (Figures 1B, C). Importantly, we saw no significant correlation between bacterial growth (as determined by OD) and host death either when we used a pilot set of $\sim 30$ isolates or with the full set of $\sim 120$ isolates (Figure 2). This suggests that host death is a specific phenomenon related to an interaction between the host and the pathogen, and does not merely result from bacterial growth.

As noted above, E. faecalis-mediated killing of C. elegans in the previously described assay depended on hydrogen peroxide production (Moy et al., 2004). Although our assay does not involve anaerobic growth (which was required for hydrogen peroxide production), we tested whether the addition of catalase to our assay would influence rates of host death. In general, we saw no decrease in killing after the addition of catalase, consistent with catalase-independent pathogenesis (Figure S1). 
A

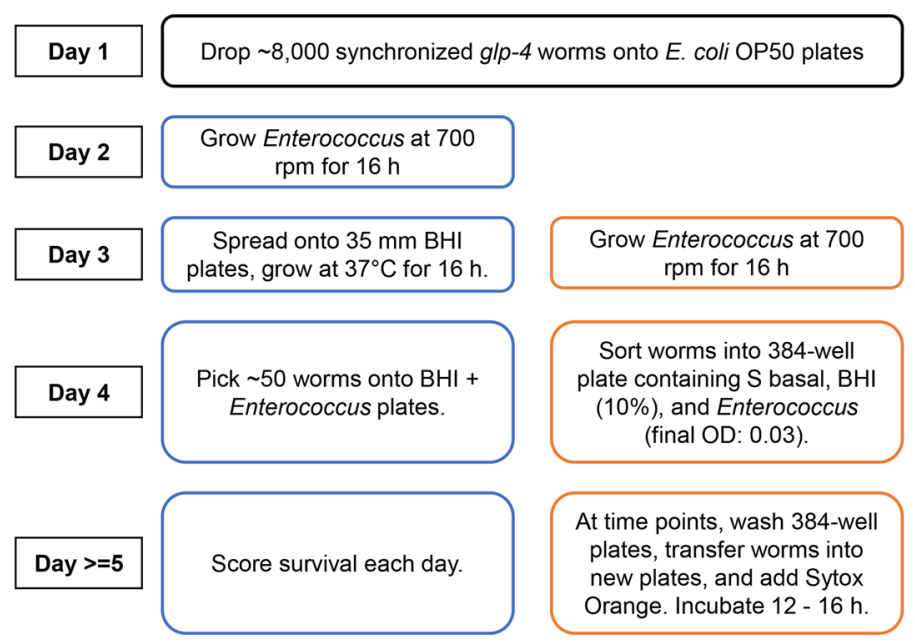

B

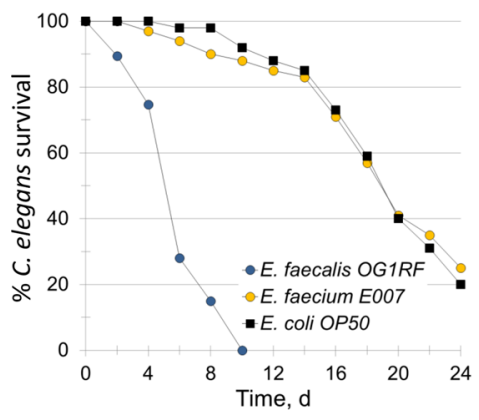

C

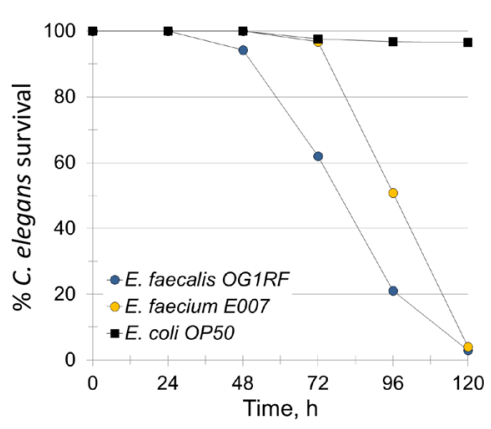

FIGURE 1 | E. faecium E007 is pathogenic to C. elegans in the Enterococcus liquid-killing assay. (A) High-throughput, liquid-based C. elegans - E. faecium killing assay pipeline (right, compared to the conventional agar-based assay (left)). (B, C) Survival curves of $C$. elegans that were exposed to E. faecalis OG1RF, E. faecium E007, or non-pathogenic E. coli OP50 either in the (B) agar-based assay or (C) under liquid conditions. Three biological replicates were performed and analyzed; representative replicates are shown for $\mathbf{( B ,} \mathbf{C})$.

A

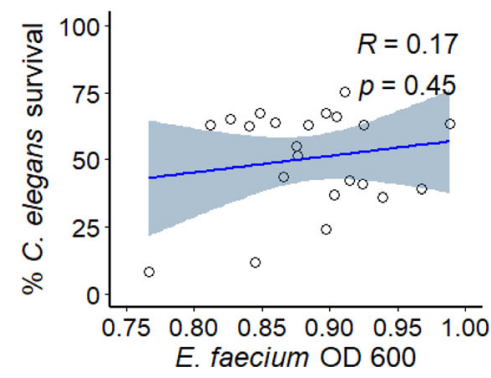

B

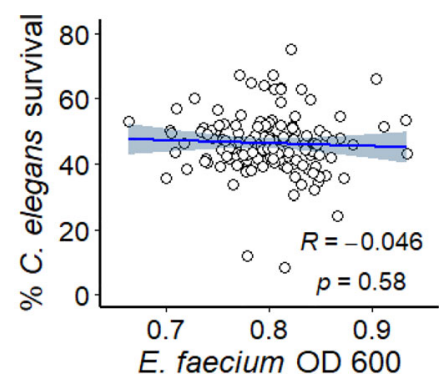

FIGURE 2 | E. faecium virulence in Enterococcus liquid killing assay does not depend upon bacterial growth. (A) Correlation plot of a subset of $\sim 30$ E. faecium strains' growth (OD 600, x-axis) against their virulence (based on C. elegans' survival, $y$-axis). (B) Correlation plot of $\sim 120$ of E. faecium strains' growth (OD 600, $x$ axis) against their virulence (based on $C$. elegans' survival, $y$-axis). Three biological replicates with $\sim 80$ worms/replicate were analyzed. The correlation plot was generated with R package ggplot2 (version 3.3.3). 


\section{PMK-1/p38 MAPK, but Not MDT-15/ MED15, Is Dispensable for C. elegans Resistance to Liquid-Based $E$. faecalis Pathogenesis}

The PMK-1/p38 MAPK pathway is arguably the most important innate immune pathway for responding to bacterial infection in $C$. elegans and has been implicated in resistance in most agar-based pathogenesis assays (Kim et al., 2002; Bolz et al., 2010; Shivers et al., 2010; Pukkila-Worley et al., 2012). In addition, pmk-1 mutation has been shown to increase sensitivity to E. faecium (Yuen and Ausubel, 2018). In contrast, the PMK-1/MAPK pathway is detrimental for survival in a liquid-based C. elegans-P. aeruginosa pathogenesis assay (Kirienko et al., 2015; Tjahjono and Kirienko, 2017). We used RNAi to knock down pmk-1 and then tested young adult worms in the liquid E. faecium assay for increased host death. As with $P$. aeruginosa, loss of PMK-1 function did not increase $E$. faecium-mediated killing in the liquid-based assay (Figures $\mathbf{3 A}, \mathbf{B})$. In contrast, disrupting PMK-1 function in the agarbased assay reduced survival (Figure 3C, S2A). These results were confirmed using a known loss-of-function mutation, pmk$1(k m 25)$ (Figure S2B).
Recently, we showed that the Mediator subunit MDT-15/ MED15 has a role in host resistance against E. faecalis (Hummell et al., 2021). To probe whether this function is retained in this liquid-based assay, we also used RNAi to knock down $m d t-15$ expression. Unlike pmk-1(RNAi), $m d t-15(R N A i)$ significantly increased host mortality in both liquid- and agar-based assays (Figure 3, S2A), suggesting that MDT-15 serves an innate immune function in these assays as well.

\section{E. faecium Strains From All Clades Showed Wide Range of Virulence}

Genome analysis of E. faecium strains indicates that they belong to at least two different phylogenetic clades: A (comprised of hospitalacquired strains and strains mostly derived from animal origins), and B (mostly human commensal strains) (Palmer et al., 2012; Galloway-Peña et al., 2012; Lebreton et al., 2013). Although earlier phylogenetic evidence suggests that clade A may be broken into two subgroups (Palmer et al., 2012; Galloway-Peña et al., 2012; Lebreton et al., 2013), a more recent, larger analysis called this conclusion into question (Raven et al., 2016). Pathogenicity of isolates from clade A1 (Figure 4A), clade A2 (Figure 4B), and clade B (Figure 4C) varied, ranging from $45-70 \%$ host mortality. Although we tested a larger

A

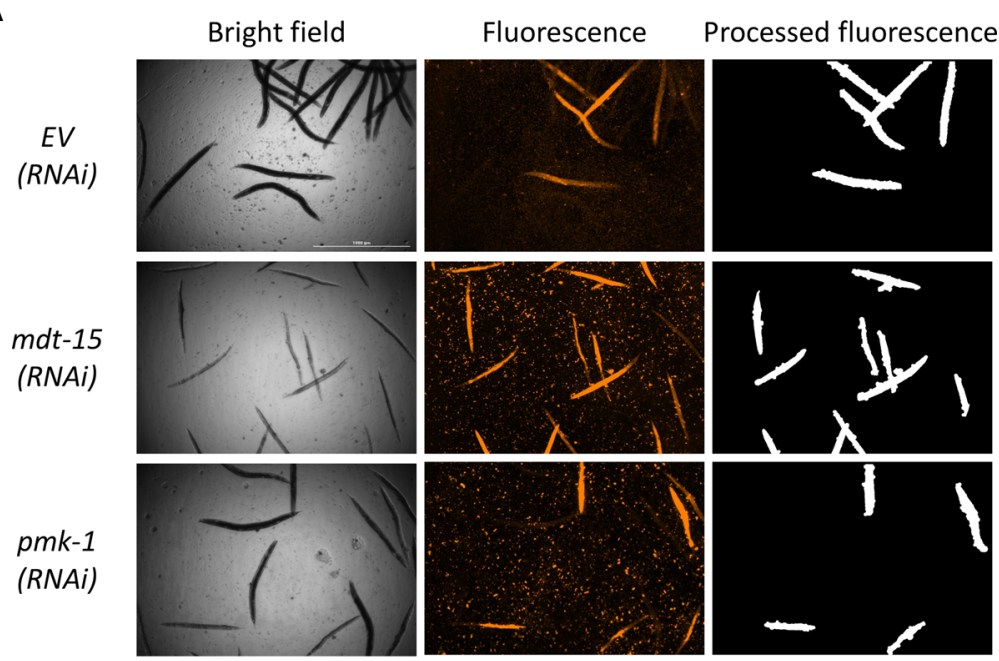

B
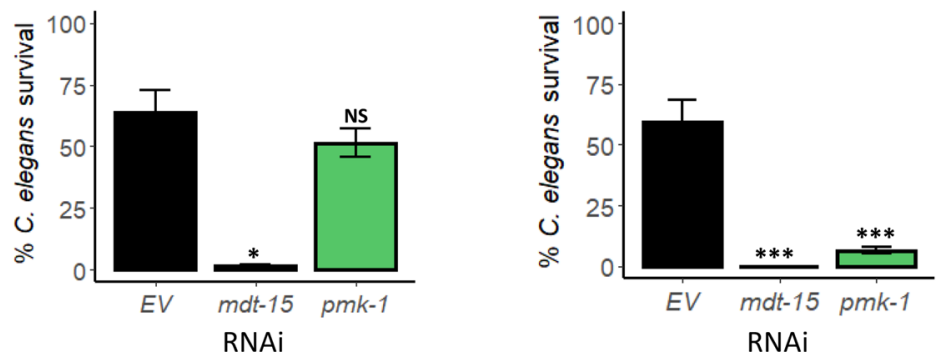

FIGURE 3 | PMK-1 did not confer resistance towards E. faecium in Enterococcus liquid-killing assay. (A) Fluorescence images and (B) quantification of C. elegans survival after exposure to $E$. faecium E007 in liquid. Worms were reared on $E$. coli expressing empty vector (EV) as control or matt-15(RNAi) or pmk-1(RNAi). (C) Quantification of $C$. elegans survival at $120 \mathrm{~h}$ exposure to E. faecium E007 in agar. Three biological replicates with $\sim 400 \mathrm{worms} /$ replicate for liquid-based assay or $\sim 180 \mathrm{worms} / \mathrm{replicate}$ for agarbased assay were analyzed. Error bars represent SEM. $p$ values were determined from Student's $t$-test. NS not significant, ${ }^{*} p<0.05,{ }^{* \star *} p<0.001$. 
A

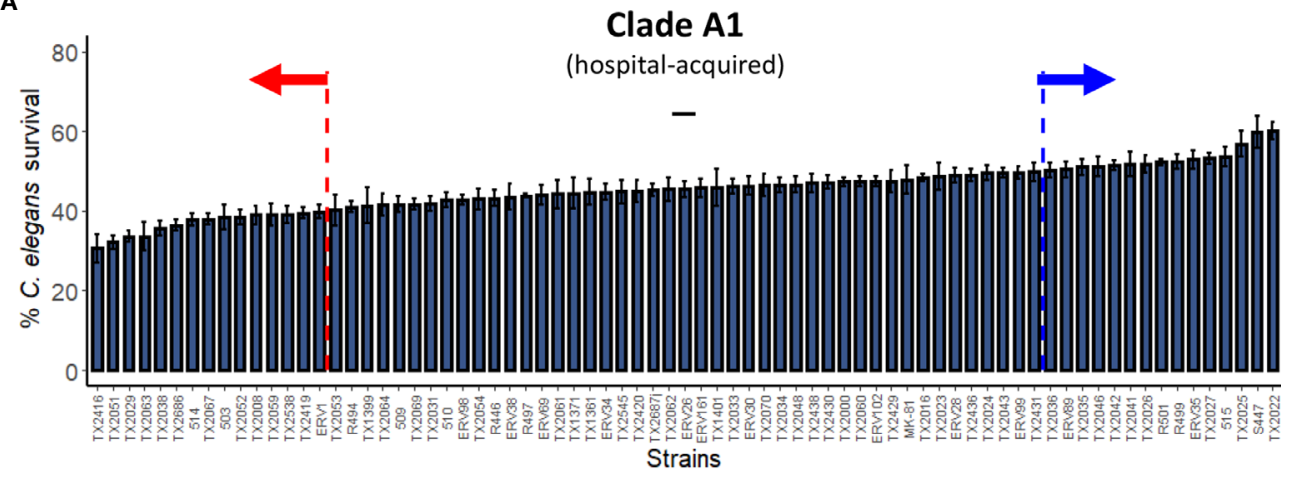

More virulent than median isolates $(p<0.05)$

B

\section{Clade A2}

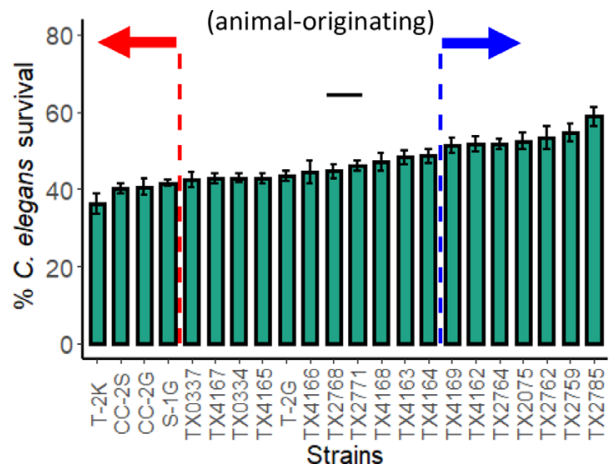

Less virulent than

median isolates $(p<0.05)$

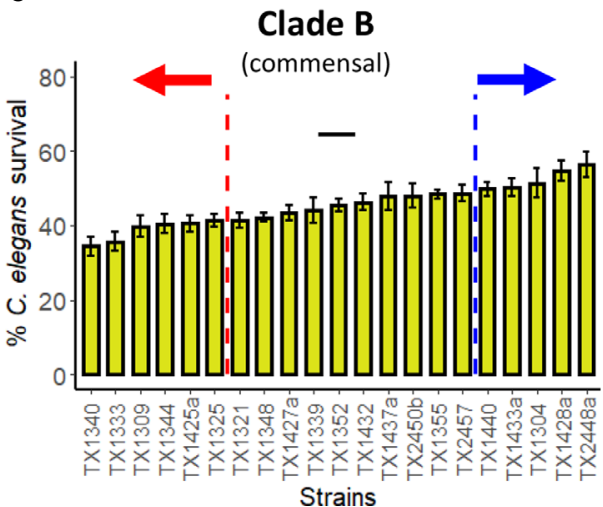

FIGURE 4 | Pathogenicity distribution of E. faecium strains belonging to Clade A1, A2, or B C. elegans were exposed to E. faecium strains belonging to (A) Clade A1, (B) Clade A2, or (C) Clade B in the liquid-killing assay. At least three biological replicates with $~ 80$ worms/replicate were analyzed. Error bars represent SEM. $p$ values were determined from Student's $t$-test against the median isolates (indicated with short black lines in the graphs). Arrows indicate all isolates that are more (red) or less (blue) virulent than the median isolates $(p<0.05)$.

number of strains from clade A1, all three clades showed similar patterns of variability in virulence. We determined median mortality for each clade and identified strains that were significantly more or less pathogenic for further analysis.

An initial analysis of the pathogenicity of $\sim 30$ isolates in our liquid-based model was performed. K-means clustering was then used to group the strains based on virulence, resulting in strong preferential grouping of $\mathrm{A} 1$ and $\mathrm{A} 2$ clades in high virulence and $\mathrm{B}$ strains in low virulence (Figure 5A). However, a more robust analysis, using a total of $\sim 100$ strains, abolished this difference, with each clade being approximately equally represented in the high- and low-virulence groups (Figure 5B).

\section{Strains With Higher Virulence Had Higher Colonization Ability in the Liquid-Based Assay}

Colonization is the first step in any infection, including bacterial infections of C. elegans with E. faecalis or E. faecium (Tan et al.,
1999; Aballay et al., 2000; Garsin et al., 2001; Kurz et al., 2003; Goh et al., 2017). In contrast, our previously reported liquid-based $P$. aeruginosa assay, which is based on intoxication rather than infection, showed relatively few bacteria in the intestine, more consistent with transit of undigested bacteria, rather than intestinal colonization (Kirienko et al., 2013). However, in liquidbased C. elegans - Candida albicans model, host colonization was observed (Rosiana et al., 2021). To test whether colonization is related to virulence in C. elegans- E. faecium liquid assay, we selected six strains from Clade A1, including the three with the highest (TX2029, TX2051, and TX2416) and the lowest (S447, TX2022, and TX2025) virulence, and infected C. elegans with them in the liquid assay (Figure 6A). After 48 hours of infection, some of the worms were harvested, washed thoroughly to remove extracorporeal bacteria, and then physically disrupted to release intestinal bacteria. Lysates were serially diluted and plated on BHI media to determine bacterial titer via CFU counting, allowing colonization to be directly measured. We found that the high-virulence strains as a 
A

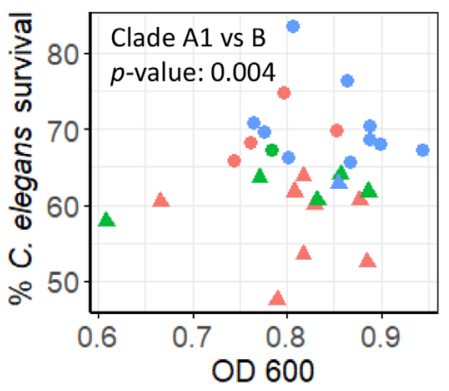

k-means cluster
B

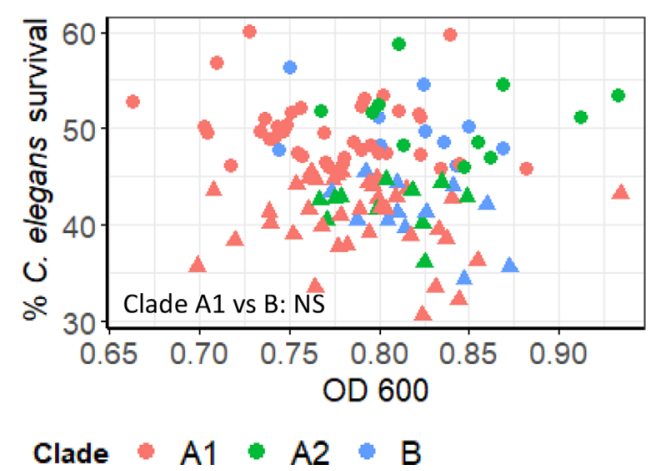

FIGURE 5 | Pathogenicity of E. faecium from distinct clades does not differ significantly. (A, B) Pathogenesis of (A) preliminary $(\sim 30)$ or $\mathbf{( B )}$ all available ( 120) E. faecium strains belonging to Clade $\mathrm{A} 1, \mathrm{~A} 2$, or $\mathrm{B}$ In (A, B), C. elegans survival (y-axis) were plotted against E. faecium OD 600 (x-axis). Clustering was performed with 'kmeans' function (R v4.0.3). All panels show pooled results from three biological replicates. $p$-values were determined from Student's $t$-test and are indicated on graphs.

group had a better ability to colonize $C$. elegans than low-virulence strains (Figure 6B and Figure S3, $p<0.001$, pooled low- and highvirulence groups). To ensure that only bacteria from the worm alimentary track, but not those that adhere to the cuticle, contributed to CFU counts, we performed the same assay except that worms were treated with gentamicin for $1 \mathrm{~h}$ after the first three
A

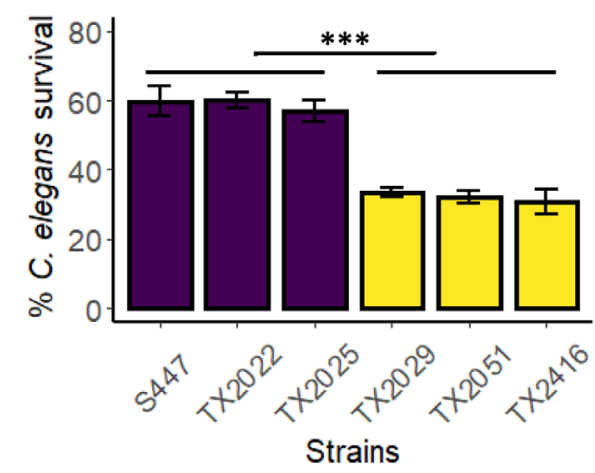

C

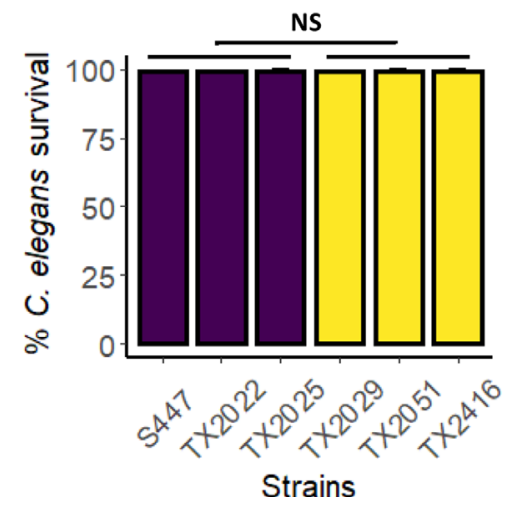

B

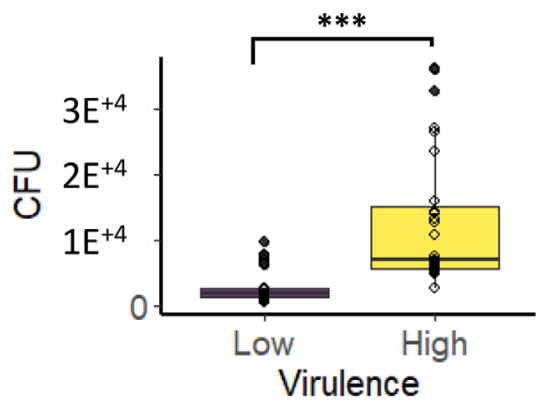

Virulence $\square$ Low $\square$ High

FIGURE 6 | Highly virulent E. faecium strains showed higher colonization, compared to low virulence strains, in the Enterococcus liquid-killing assay. Six E. faecium strains (three with the highest virulence in the liquid-based assay and three with the lowest virulence) were exposed to $C$. elegans in liquid-based assay. Panel (A) shows strain pathogenesis (C. elegans survival), while panel (B) shows pooled average of CFU from C. elegans gut after colonization. CFU counts for individual strains are in Figure S3. (C) The killing ability of bacteria-free filtrates (from isolates in panel A) was examined after $100 \mathrm{~h}$ incubation with $C$. elegans. Three biological replicates with $\sim 60$ worms/replicate for CFU assay or $\sim 400$ worms/replicate for pathogenesis assay were analyzed. Error bars represent SEM. $p$-values were determined from Student's $t$-test between pooled low- and high virulence groups. NS not significant, ${ }^{\star \star \star} p<0.001$. 
washes (Figure S4). Worms were then incubated with $40 \mu \mathrm{M}$ acridine orange, a dye commonly used to stain bacteria (Neeraja et al., 2017). Fluorescent imaging confirmed that bacteria were only detected in the worms' gastrointestinal tract, both for gentamicintreated and untreated sub-populations, but not on the cuticles (Figures S4A, B). These results suggested that the ability to persist in C. elegans gut may be an important contributor to $E$. faecium virulence in this liquid assay.

In a previously-published liquid-based C. elegans pathosystem, we showed that toxin secretion, particularly of the siderophore pyoverdine, was very important for host killing by $P$. aeruginosa (Kirienko et al., 2015; Kang et al., 2018). In this case, spent media or purified pyoverdine were capable of killing worms even in the absence of live bacteria (Figure S5). To test the ability of secreted factors to affect host survival during E. faecium infection, the same panel of six $E$. faecium isolates was grown under assay conditions, but in the absence of worms, for four days. Then bacteria-free filtrates were produced and C. elegans were exposed to them for $\sim 100 \mathrm{~h}$ (Figure 6C). Virtually no host death was observed, suggesting that live bacteria and host-pathogen interactions are the driving force of pathogenesis in this assay.

\section{Some fms genes Are Important for Pathogenesis in the C. elegans - E. faecium Liquid Killing Assay}

E. faecium infection is a multifactorial process that includes attachment, colonization, and biofilm formation. To determine which virulence factors are important for killing in liquid, we measured the pathogenicity of available E. faecium strains harboring deletions of targeted virulence factors (Table 1), including genes from the $g l s$ (Choudhury et al., 2011), fms (Sillanpää et al., 2008; Sillanpää et al., 2009; Sillanpää et al., 2010), wxl (Galloway-Peña et al., 2015), and ccpA (Somarajan et al., 2014) genes and gene families.

Deletion of genes encoding general stress proteins (Gls) gls33$g l s B$ alone or doubled with $g l s 20-g l s B 1$ did not appear to affect host survival (Figure S6A). This also seemed to be the case with mutants harboring deletions of genes in the WxL loci (GallowayPeña et al., 2015). The WxL loci, are a series of three loci that each encode between 3 and 6 genes, including peptidoglycanbinding proteins (Brinster et al., 2007). Three strains with a single mutation each and one strain with triple mutations of the WxL loci did not significantly alter worms' survival as compared to the parental strain TX0082 (Figure S6B).

On the other hand, the E. faecium surface proteins (Fms) of the MSCRAMM (microbial surface component recognizing adhesive matrix molecules) family were more likely to be involved in pathogenesis in this E. faecium - C. elegans killing assay. fms genes are tightly associated with E. faecium clinical isolates (Sillanpää et al., 2008). We tested multiple mutants with one (scm, acm, or fms15), two $\left(\Delta f m s 21-20, a m^{\mathrm{Y} 176 \mathrm{~A}, \mathrm{~F} 192 \mathrm{~A}} \Delta \mathrm{scm}\right.$, and $\Delta f m s 11 \Delta s c m)$, or three genes mutated $\left(a^{\mathrm{cm}}{ }^{\mathrm{Y} 176 \mathrm{~A}, \mathrm{~F} 192 \mathrm{~A}}\right.$ $\Delta f m s 18 \Delta s c m, \Delta e b p A B C f m$, and $\left.\mathrm{acm}^{\mathrm{Y} 176 \mathrm{~A}, \mathrm{~F} 192 \mathrm{~A}} \Delta \mathrm{fms} 11 \Delta \mathrm{scm}\right)$. Among the strains tested, the single deletion of $f m s 15$, an adhesin gene, significantly reduced E. faecium virulence (Figure 7A). Moreover, triple mutation of $\mathrm{acm}^{\mathrm{Y} 176 \mathrm{~A}, \mathrm{~F} 192 \mathrm{~A}} \Delta \mathrm{fms} 11 \Delta \mathrm{scm}$ and deletion of pilus subunit proteins encoded by the three-gene locus ebpABCfm (fms1-5-9) reduced pathogenicity as well (Figure 7B). As the MSCRAMMs play a role in host-pathogen adherence, these results corroborated our finding that colonization and persistence in $C$. elegans gut is important for virulence.

Interestingly, deletion of $c c p A$, a regulator of carbon catabolite repression that affects $E$. faecium growth and is important for virulence (Somarajan et al., 2014), increased pathogenicity (Figure 7B). This result was unexpected as $\operatorname{ccp} A$ deletion mutant showed reduced biofilm formation, growth, and attenuated infection in endocarditis model (Somarajan et al., 2014).

\section{Genome Analysis of Clade A1 Strains}

To further understand E. faecium virulence mechanisms, we obtained whole genome sequencing data for eight strains in the
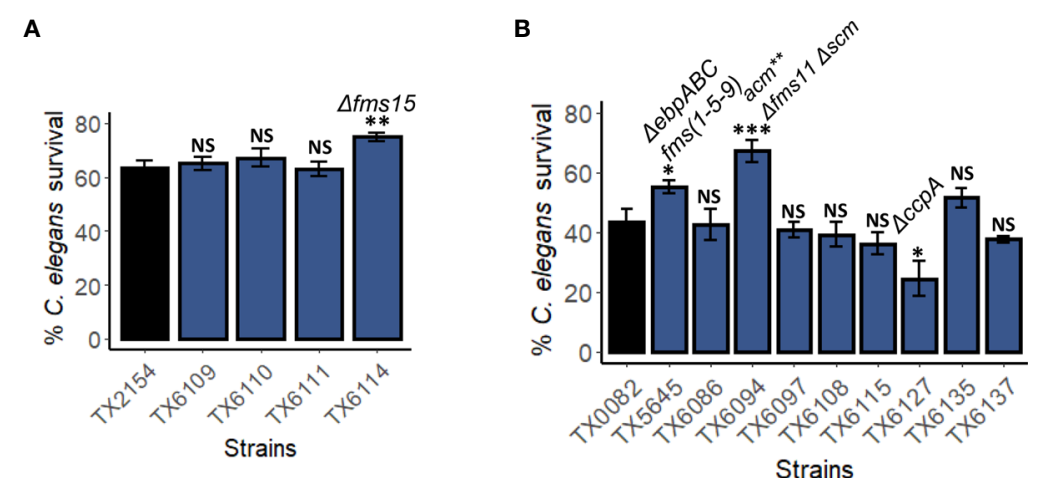

FIGURE 7 | Genes encoding surface proteins and pili are important for pathogenesis in the C. elegans - E. faecium liquid-killing assay. Pathogenicity of several strains harboring mutations in (A) fms10 (scm), acm, fms18, or fms15, or (B) fms, scm, wxl, or ccpA were assayed in the C. elegans - E. faecium liquid-based pathosystem. Three biological replicates with $\sim 400$ worms/replicate were analyzed. Error bars represent SEM. $p$ values were determined from Student's $t$-test. NS not significant, ${ }^{\star} p<0.05,{ }^{\star \star} p<0.01,{ }^{\star \star \star} p<0.001$. 
higher virulence and six strains in the lower virulence groups. Strains from Clade A1 were selected for this analysis as they are most commonly associated with human infection. Phylogenetic tree construction showed close relationships among the fourteen strains, except for TX2046 (lower virulence) and TX2051 (higher virulence) (Figure 8A).

Genomic analysis showed that TX2046 contained two frameshift mutations: a one nucleotide insertion in $\operatorname{sorB}$ (a PTS system sorbose-specific EIIB component) and another in mazE (the gene encoding the MazE Type II antitoxin). TX2051 acquired a frameshift in araQ ( $L$-arabinose transport system permease protein) and a stop codon in wecA (UDP-Nacetylgalactosamine-undecaprenyl-phosphate $\mathrm{N}$ acetylgalactosamine phosphotransferase).

Toxin-antitoxin pairs are commonly found in bacterial pathogens, and mutation of the antitoxin mazE would result in greatly diminished bacterial survival. We performed growth analysis of the OD 600 for the two strains TX2046 and TX2051. We observed differences in growth rates, with TX2051 growing faster than TX2046 (Figure 8B). Thus, TX2051 might be more virulent due to faster growth and colonization rate. This result also showed that, at least in selected cases, pathogenicity may depend on bacterial growth. However, these two parameters did not correlate in a larger group (Figure 8B and Figure S3).

\section{The Liquid-Based E. faecium Model Recapitulates Virulence Observed in a Murine Peritonitis Model}

Most importantly, our C. elegans - E. faecium pathogenesis model recapitulated virulence in a murine peritonitis model. Two strains that differ in origin, TX0016 [or DO, an endocarditis isolate with one of the first E. faecium genomes sequenced (Qin et al., 2012)] and TX1330 [a community-derived isolate (Qin et al., 2012)] were used to infect $C$. elegans or mice. Pathogenesis was noticeably reduced in TX0016 strain compared to TX1330 in both C. elegans (Figure 9A) and mice (Figure 9B).

\section{DISCUSSION}

In this study, we developed a C. elegans - E. faecium liquid-based pathogenesis assay that includes a lethal infection. Previous tests of E. faecium-infected C. elegans showed that the bacterium could effectively colonize the intestine of C. elegans, and even

A

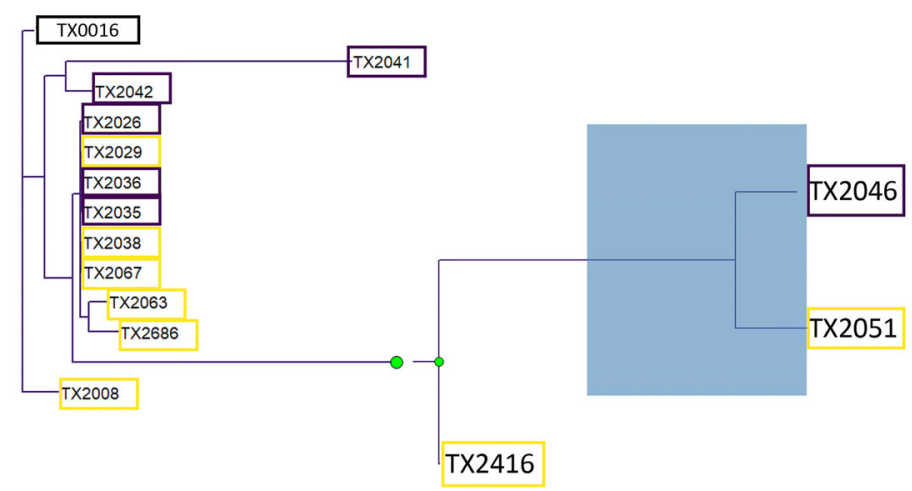

B

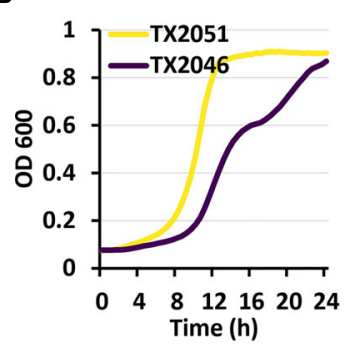

C

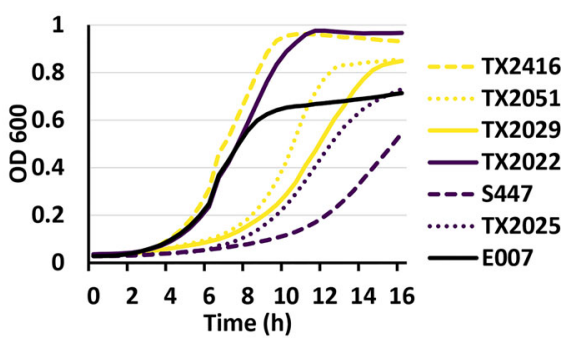

Virulence $\square$ Low $\square$ High

FIGURE 8 | Genomic analysis showed a relationship between high- and low virulence strains. (A) A maximum-likelihood phylogenetic tree of eight strains belonging to the highest virulence group (yellow), six strains belonging to the lowest virulence group (purple), and a reference strain (TX0016, black). The phylogenetic tree was created by performing roary (Galaxy version 3.13.0), followed by RAxML (Galaxy version 1.0.0), and visualized by using treeio (v1.14.3) and ggtree (v2.4.1) packages (RStudio, R version 4.0.3). (B) Kinetic growth measurement or optical densities (OD 600) of TX2046 and TX2051 in BHI media. (C) Kinetic growth measurement or optical densities (OD 600) of three high- (TX2416, TX2051, TX2029) and three low-virulence isolates (TX2022, S447, TX2025), and reference strain E007 in BHI media. For $(\mathbf{B}, \mathbf{C})$, three biological replicates were performed and analyzed; a representative replicate is shown. 


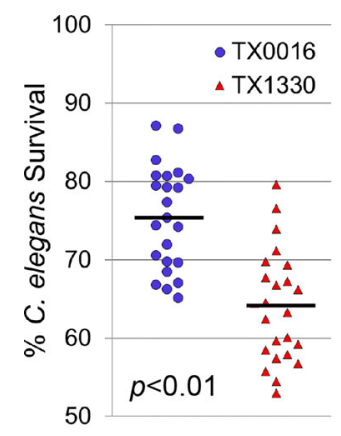

B

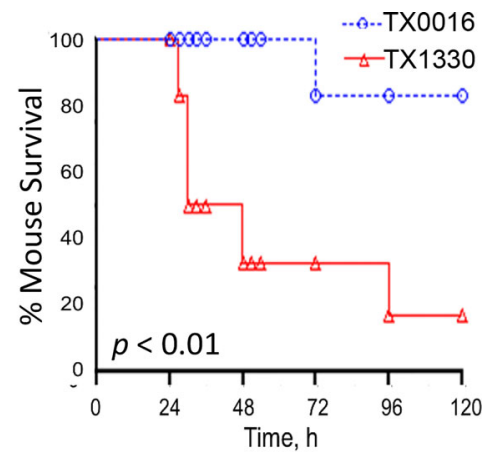

FIGURE 9 | The C. elegans - E. faecium pathogenesis model recapitulates E. faecium virulence. (A, B) Survival curves of (A) C. elegans or (B) mice that were exposed to two E. faecium strains TX0016 and TX1330. All panels show results from pooled biological replicates (each dot in $\mathbf{A}$ represents a group of 20 worms). $p$ values were determined from Student's $t$-test and are indicated on graphs.

trigger an immune response (Garsin et al., 2001; Yuen and Ausubel, 2018), but the worms retained nearly normal life spans. Disrupting host immune effectors caused worms to succumb, indicating that this defense is productive. Interestingly, the death that we observed suggests that either a different pathogenic mechanism is used in this assay or that the normal defense mechanism has been compromised. This conclusion is bolstered by our observation that PMK-1/p38 MAPK innate immune pathway activity is not involved in survival.

This E. faecium screen was developed from our previously published C. elegans - P. aeruginosa liquid-killing assay (Kirienko et al., 2013) and median survival was typically 4 days post infection. This model allows high-throughput screens to be conducted for studying E. faecium virulence, an advantage other current model systems do not offer (Garsin et al., 2014; Goh et al., 2017). This enables identification of virulence factors by screening for increased host survival compared to wildtype bacteria.

Most importantly, our C. elegans model recapitulated features of virulence observed in a murine-E. faecium model. Despite their evolutionary distance, $C$. elegans retains many important features of the innate immune system (e.g., p38 MAPK, Nrf2, FOXO pathways), host responses (e.g., lysozymes, lectins, other effectors production), and metabolic adjustments seen in mammals (Irazoqui et al., 2010; Pukkila-Worley and Ausubel, 2012; Battino et al., 2018). Admittedly, we saw considerable differences in sensitivity in C. elegans vs mammalian models when using known attenuated mutants. However, the rapid generation time and low cost will allow this assay to be further refined to more accurately reflect mammalian infections. Meanwhile, this host-pathogen interaction model is capable of probing host and pathogen genetic factors that influence virulence. In addition, the small size of $C$. elegans allows this assay to be performed in 384-well plates, making it amenable for high-throughput screening and medicinal chemistry approaches.

Once initial studies with our screen are complete, a potential model system to validate the results of these screens is an organoid culture system. This culture system is currently thriving and many types, including intestinal and lung organoids, have been successfully established (Dutta and Clevers, 2017). Organoid systems can provide additional insights into the interplay between E. faecium and human bodies, particularly the inflammatory responses. The idea of finding therapeutics that may mitigate pathogenesis or stimulate hosts' immune systems and potentially shift this pathogen back toward a commensal state is a tantalizing prospect. Since the rise and spread of resistance to treatment with these effects is likely to be slower than for conventional antimicrobials, this might also boost our ability to treat these infections moving forward.

Interestingly, we found no correlation between the initial bacterial titer of E. faecium and pathogenesis towards C. elegans in this system. Although some strains, like TX2051 and TX2046 (the latter is missing the MazE antitoxin, and shows slower bacterial growth), showed a correlation between faster growth and higher virulence, there was no overall trend. This is consistent with our observation that secreted virulence factors do not seem responsible and that colonization is required, as shown by diminished pathogenesis by the fms 15 mutant.

This finding, and the broader context of efficient intestinal colonization with E. faecium in this assay, was unexpected, given our previous results with a $C$. elegans $-P$. aeruginosa assay that has been utilized by our lab for a number of years. In the $P$. aeruginosa liquid pathogenesis assay, the living bacteria present in the intestine appear to be transient and have escaped the digestive process, but are not establishing a significant colonization. Consequently, isolates or mutants that would be attenuated on agar may have full virulence in liquid, and vice versa. In the case of $P$. aeruginosa, growth on agar (or in the host) leads to high bacterial density and key involvement of quorumsensing pathways (Feinbaum et al., 2012). In liquid, growth is limited, quorum sensing has no role, and production of the siderophore pyoverdine drives a lethal host intoxication (Kirienko et al., 2015; Kang et al., 2018; Kang and Kirienko, 2020). 
A recently published analysis of the role of Candida albicans adhesins in virulence using a liquid-based $C$. elegans model (Rosiana et al., 2021) reveals a third possibility. In this model, like the $P$. aeruginosa and E. faecium models, greater colonization is observed in an agar-based assay than a liquid assay, although colonization still occurs in liquid, like with $E$. faecium. Unlike either previous model however, pathogenesis and colonization are inversely correlated here. From comparing these systems, it is obvious that host-pathogen interactions are dynamic, depending upon virulence factors regulated by both participants, and that careful study is needed in each case.

Host defenses are also clearly context- and pathogendependent, and PMK-1/p38 MAPK can range from essential ( $P$. aeruginosa infection on agar, E. faecium on agar) to dispensable (E. faecium infection in liquid) to detrimental ( $P$. aeruginosa intoxication in liquid). Involvement of the Mediator complex subunit MDT-15/Med15 was recently shown to be required for increased survival of $C$. elegans exposed to both Gram-negative and Gram-positive bacteria (e.g., P. aeruginosa and $E$. faecalis) in liquid when treated with immune-stimulatory compounds (Hummell et al., 2021). Here mdt-15/Med15 knockdown resulted in dramatic susceptibility of C. elegans to E. faecium infection in liquid. This indicates that the involvement of Mediator complex into innate immunity may be a widespread phenomenon.

In this study, we tested $\sim 120$ E. faecium strains isolated from various clinical and environmental samples from many different geographical locations. Most of the strains belong to Clade A1, a group that includes members that are tightly associated with nosocomial infections and are notorious for their hypermutability, acquisition of assorted pathogenic determinants, and acquisition of resistance mechanisms (Lebreton et al., 2013; Freitas et al., 2018). Clade A and Clade B (commensal) have been estimated to have diverged approximately 3000 years ago (Lebreton et al., 2013; Raven et al., 2016), with the former carrying more genetic elements encoding $\mathrm{ABC}$ transporters for antibiotic transport and phosphotransferase systems for utilization of carbohydrates with a non-dietary origin. These features are predicted to make Clade A more likely to be resistant to antimicrobials and more pathogenic (Lebreton et al., 2013). To our surprise, however, each clade showed similar distribution of virulence, suggesting that each group has potential for threatening public health. Indeed, recent evidence showed that infection with Clade B isolates is increasingly frequent (Lebreton et al., 2018), much as was observed for E. faecium in general over the last 50 years. Troublingly, antibiotic resistance is spreading rapidly within populations in Clade B, and hybrid strains of Clade A1 and B are increasingly common (Galloway-Peña et al., 2012; Lebreton et al., 2013).

Recent studies profiling putative virulence markers in various E. faecium isolates with diverse origins agreed with this finding. Uneven distributions of several of these virulence markers were observed within the three clades (Qin et al., 2012; Freitas et al., 2018). While Clade A1 isolates carry more putative virulence markers than Clade A2 or B, most virulence markers are present in all three clades, including $a c m, s c m, f m s 15, f m s 21, e b p B C$, and $\mathrm{WxL}$ genes, indicating their importance for host colonization (Freitas et al., 2018). Meanwhile, fms11, fms18, fms20, and ebpA are mostly found within Clade A1. The presence of complete pili gene clusters, e.g., $e b p A B C_{f m}$, however, is tightly associated with ampicillin-resistant clinical isolates (Qin et al., 2012; Freitas et al., 2018).

We validated our screen by confirming the above-mentioned adhesion-related virulence factors that play a role in E. faecium pathogenesis. Although most single mutations (except for $\Delta f m s 15)$ lacked a clear phenotype, this is likely due to genetic redundancy. Combining multiple mutations in a common pathway reduced pathogenesis. For example, combining mutations in $e b p A B C_{f m}$ (or empABC or fms1-5-9) and acmfms11-scm of E. faecium considerably limited lethality. These surface proteins are enriched in clinical isolates and aid in attachment to extracellular matrix proteins (Sillanpää et al., 2008; Hendrickx et al., 2008; Sillanpää et al., 2009; Sillanpää et al., 2010; Qin et al., 2012; Montealegre et al., 2016). The loss of ebpABCfm significantly attenuated biofilm formation and reduced virulence in a murine UTI model (Montealegre et al., 2016). This form of redundancy suggests that it will be especially critical to target regulatory mechanisms in E. faecium when developing future therapies, so that this redundancy does not compromise treatment effect.

\section{DATA AVAILABILITY STATEMENT}

The raw data supporting the conclusions of this article will be made available by the authors, without undue reservation.

\section{ETHICS STATEMENT}

The animal study was reviewed and approved by Animal Welfare committee, University of Texas Health Science Center at Houston.

\section{AUTHOR CONTRIBUTIONS}

AR performed a majority of the experiments. ET performed some of the experiments. AR and ET wrote the first draft of the manuscript. BM and KS contributed to conception of the study and provided E. faecium isolates and mouse data. KS participated in manuscript writing. $\mathrm{BH}$ performed sequencing experiments. NK performed overall design of the study, contributed to data analysis and manuscript writing and editing. All authors contributed to the article and approved the submitted version.

\section{FUNDING}

This work was supported by a John S. Dunn Foundation Award to NK and BM and NIH NIGMS Award R35GM129294 to NK. 
Funders had no role in study design, data collection or analysis, decision to publish or preparation of the manuscript.

\section{ACKNOWLEDGMENTS}

E. faecium E007 was a gift from Dr. Danielle Garsin. C. elegans strains were obtained from CGC which is funded by NIH Office of Research Infrastructure Programs (P40 OD010440). The

\section{REFERENCES}

Aballay, A., Yorgey, P., and Ausubel, F. (2000). Salmonella Typhimurium Proliferates and Establishes a Persistent Infection in the Intestine of Caenorhabditis Elegans. Curr. Biol. 10 (23), 1539-1542. doi: 10.1016/S09609822(00)00830-7

Anderson, Q. L., Revtovich, A. V., and Kirienko, N. V. (2018). A High-throughput, High-Content, Liquid-based C. Elegans Pathosystem. J. Vis. Exp. JoVE 137), 58068. doi: 10.3791/58068

Arias, C. A., Panesso, D., Singh, K. V., Rice, L. B., and Murray, B. E. (2009). Cotransfer of Antibiotic Resistance Genes and a hylEfm-containing Virulence Plasmid in Enterococcus Faecium. Antimicrob. Agents Chemother. 53 (10), 4240-4246. doi: 10.1128/AAC.00242-09

Battino, M., Giampieri, F., Pistollato, F., Sureda, A., de Oliveira, M. R., Pittala, V., et al. (2018). Nrf2 as Regulator of Innate Immunity: A Molecular Swiss Army Knife! Biotechnol. Adv. 36 (2), 358-370. doi: 10.1016/j.biotechadv.2017.12.012

Bolz, D. D., Tenor, J. L., and Aballay, A. (2010). A Conserved PMK-1/P38 MAPK is Required in Caenorhabditis Elegans Tissue-specific Immune Response to Yersinia Pestis Infection. J. Biol. Chem. 285 (14), 10832-10840. doi: 10.1074/ jbc.M109.091629

Bonazzetti, C., Morena, V., Giacomelli, A., Oreni, L., Casalini, G., Galimberti, L., et al. (2021). 'Unexpectedly High Frequency of Enterococcal Bloodstream Infections in Coronavirus Disease 2019 Patients Admitted to an Italian Icu: An Observational Study'. Crit. Care Med. 49 (1), e31-e40. doi: 10.1097/ CCM.0000000000004748

Brinster, S., Furlan, S., and Serror, P. (2007). 'C-Terminal WxL Domain Mediates Cell Wall Binding in Enterococcus Faecalis and Other Gram-Positive Bacteria'. J. Bacteriol. 189 (4), 1244-1253. doi: 10.1128/JB.00773-06

CDC (2019). Antibiotic Resistance Threats in the United States (Atlanta, GA: U.S. Department of Health and Human Services, CDC). Available at: http://dx.doi. org/10.15620/cdc:82532.

Choudhury, T., Singh, K. V., Sillanpaa, J., Nallapareddy, S. R., and Murray, B. E. (2011). Importance of Two Enterococcus Faecium Loci Encoding Gls-like Proteins for In Vitro Bile Salts Stress Response and Virulence. J. Infect. Dis. 203 (8), 1147-1154. doi: 10.1093/infdis/jiq160

Conery, A. L., Larkins-Ford, J., Ausubel, F. M., and Kirienko, N. V. (2014). HighThroughput Screening for Novel Anti-Infectives Using a C. Elegans Pathogenesis Model. Curr. Protoc. Chem. Biol. 6 (1), 25-37. doi: 10.1002/ 9780470559277.ch130160

Coque, T. M., Patterson, J. E., Steckelberg, J. M., and Murray, B. E. (1995). Incidence of Hemolysin, Gelatinase, and Aggregation Substance Among Enterococci Isolated From Patients With Endocarditis and Other Infections and From Feces of Hospitalized and Community-Based Persons. J. Infect. Dis. 171 (5), 1223-1229. doi: 10.1093/infdis/171.5.1223

Davis, E., Hicks, L., Ali, I., Salzman, E., Wang, J., Snitkin, E., et al. (2020). Epidemiology of Vancomycin-Resistant Enterococcus Faecium and Enterococcus Faecalis Colonization in Nursing Facilities. Open Forum Infect. Dis. 7 (1), ofz553. doi: 10.1093/ofid/ofz553

Diaz, L., Tran, T. T., Munita, J. M., Miller, W. R., Rincon, S., Carvajal, L. P., et al. (2014). Whole-Genome Analyses of Enterococcus Faecium Isolates With Diverse Daptomycin Mics. Antimicrob. Agents Chemother. 58 (8), 45274534. doi: 10.1128/AAC.02686-14

Dutta, D., and Clevers, H. (2017). Organoid Culture Systems to Study HostPathogen Interactions. Curr. Opin. Immunol. 48, 15-22. doi: 10.1016/ j.coi.2017.07.012 authors would like to thank the Kirienko lab members for productive discussions.

\section{SUPPLEMENTARY MATERIAL}

The Supplementary Material for this article can be found online at: https://www.frontiersin.org/articles/10.3389/fcimb.2021.667327/ full\#supplementary-material

Feinbaum, R. L., Urbach, J. M., Liberati, N. T., Djonovic, S., Adonizio, A., Carvunis, A. R., et al. (2012). Genome-Wide Identification of Pseudomonas Aeruginosa Virulence-Related Genes Using a Caenorhabditis Elegans Infection Model. PloS Pathog. 8 (7), e1002813. doi: 10.1371/journal.ppat.1002813

Freitas, A., Tedim, A., Novais, C., Coque, T., and Peixe, L. (2018). Distribution of Putative Virulence Markers in Enterococcus Faecium: Towards a Safety Profile Review. J. Antimicrob. Chemother. 73 (2), 306-319. doi: 10.1093/jac/ dkx387

Galloway-Peña, J., Liang, X., Singh, K., Yadav, P., Chang, C., La, R. S. L., et al. (2015). The Identification and Functional Characterization of WxL Proteins From Enterococcus Faecium Reveal Surface Proteins Involved in Extracellular Matrix Interactions. J. Bacteriol. 197 (5), 882-892. doi: 10.1128/JB.02288-14

Galloway-Peña, J., Roh, J., Latorre, M., Qin, X., and Murray, B. (2012). Genomic and SNP Analyses Demonstrate a Distant Separation of the Hospital and Community-Associated Clades of Enterococcus Faecium. PloS One 7 (1), e30187. doi: 10.1371/journal.pone.0030187

Gao, W., Howden, B. P., and Stinear, T. P. (2018). Evolution of Virulence in Enterococcus Faecium, a Hospital-Adapted Opportunistic Pathogen. Curr. Opin. Microbiol. 41, 76-82. doi: 10.1016/j.mib.2017.11.030

Garsin, D., Frank, K. L., Silanpää, J., Ausubel, F. M., Hartke, A., Shankar, N., et al. (2014). Pathogenesis and Models of Enterococcal Infection. In: Gilmore MS, Clewell DB, Ike Y, Shankar N, editors. Enterococci: From Commensals to Leading Causes of Drug Resistant Infection [Internet]. Boston: Massachusetts Eye and Ear Infirmary.

Garsin, D., Sifri, C., Mylonakis, E., Qin, X., Singh, K., Murray, B., et al. (2001). A Simple Model Host for Identifying Gram-positive Virulence Factors. Proc. Natl. Acad. Sci. United States America 98 (19), 10892-10897. doi: 10.1073/pnas.191378698

Goh, H., Yong, M., Chong, K., and Kline, K. (2017). Model Systems for the Study of Enterococcal Colonization and Infection. Virulence 8 (8), 1525-1562. doi: 10.1080/21505594.2017.1279766

Heikens, E., Bonten, M. J., and Willems, R. J. (2007). Enterococcal Surface Protein Esp is Important for Biofilm Formation of Enterococcus Faecium E1162. J. Bacteriol. 189 (22), 8233-8240. doi: 10.1128/JB.01205-07

Heimer, S. R., Yamada, A., Russell, H., and Gilmore, M. (2010). Response of Corneal Epithelial Cells to Staphylococcus Aureus. Virulence 1 (4), 223-235. doi: 10.4161/viru.1.4.11466

Hendrickx, A., Bonten, M., van Luit-Asbroek, M., Schapendonk, C., Kragten, A., and Willems, R. (2008). Expression of Two Distinct Types of Pili by a HospitalAcquired Enterococcus Faecium Isolate. Microbiol. (Reading England) 154 (Pt 10), 2112-3223. doi: 10.1099/mic.0.2008/020891-0

Hendrickx, A., van Luit-Asbroek, M., Schapendonk, C., van Wamel, W., Braat, J., Wijnands, L., et al. (2009). SgrA, a Nidogen-Binding LPXTG Surface Adhesin Implicated in Biofilm Formation, and EcbA, a Collagen Binding MSCRAMM, are Two Novel Adhesins of Hospital-Acquired Enterococcus Faecium. Infect. Immun. 77 (11), 5097-5106. doi: 10.1128/IAI.00275-09

Hendrickx, A., van Wamel, W., Posthuma, G., Bonten, M., and Willems, R. (2007). Five Genes Encoding Surface-Exposed LPXTG Proteins are Enriched in Hospital-Adapted Enterococcus Faecium Clonal Complex 17 Isolates. J. Bacteriol. 189 (22), 8321-8332. doi: 10.1128/JB.00664-07

Hummell, N., Revtovich, A., and Kirienko, N. (2021). Novel Immune Modulators Enhance Caenorhabditis Elegans Resistance to Multiple Pathogens. mSphere 6 (1), e00950-20. doi: 10.1128/mSphere.00950-20

Huycke, M., Sahm, D., and Gilmore, M. (1998). Multiple-Drug Resistant Enterococci: The Nature of the Problem and an Agenda for the Future. Emerg. Infect. Dis. 4 (2), e00950-e00920. doi: 10.3201/eid0402.980211 
Irazoqui, J. E., Urbach, J. M., and Ausubel, F. M. (2010). Evolution of Host Innate Defence: Insights From Caenorhabditis Elegans and Primitive Invertebrates. Nat. Rev. Immunol. 10 (1), 47-58. doi: 10.1038/nri2689

Iwen, P., Kelly, D., Linder, J., Hinrichs, S., Dominguez, E., Rupp, M., et al. (1997). Change in Prevalence and Antibiotic Resistance of Enterococcus Species Isolated From Blood Cultures Over an 8-Year Period. Antimicrob. Agents Chemother. 41 (2), 494-495. doi: 10.1128/AAC.41.2.494

Kafil, H., and Asgharzadeh, M. (2014). Vancomycin-Resistant Enteroccus Faecium and Enterococcus Faecalis Isolated From Education Hospital of Iran. Maedica 9 (4), 323-327.

Kamath, R. S., Fraser, A. G., Dong, Y., Poulin, G., Durbin, R., Gotta, M., et al. (2003). Systematic Functional Analysis of the Caenorhabditis Elegans Genome Using Rnai. Nature 421 (6920), 231-237. doi: 10.1038/nature01278

Kampmeier, S., Tönnies, H., Correa-Martinez, C., Mellmann, A., and Schwierzeck, V. (2020). A Nosocomial Cluster of Vancomycin Resistant Enterococci Among COVID-19 Patients in an Intensive Care Unit. Antimicrob. Resist. Infect. Control 9 (1), 154. doi: 10.1186/s13756-020-00820-8

Kang, D., and Kirienko, N. V. (2020). An In Vitro Cell Culture Model for Pyoverdine-Mediated Virulence. Pathogens 10 (1), 9. doi: 10.3390/ pathogens10010009

Kang, D., Kirienko, D. R., Webster, P., Fisher, A. L., and Kirienko, N. V. (2018). Pyoverdine, a Siderophore From Pseudomonas Aeruginosa, Translocates Into C. Elegans, Removes Iron, and Activates a Distinct Host Response. Virulence 9 (1), 804-817. doi: 10.1080/21505594.2018.1449508

Kim, D. H., Feinbaum, R., Alloing, G., Emerson, F. E., Garsin, D. A., Inoue, H., et al. (2002). A Conserved P38 MAP Kinase Pathway in Caenorhabditis Elegans Innate Immunity. Science 297 (5581), 623-626. doi: 10.1126/ science. 1073759

Kim, G. H., Rosiana, S., Kirienko, N. V., and Shapiro, R. S. (2020). A Simple Nematode Infection Model for Studying Candida Albicans Pathogenesis. Curr. Protoc. Microbiol. 59 (1), el14. doi: 10.1002/cpmc.114

Kim, D. S., Singh, K. V., Nallapareddy, S. R., Qin, X., Panesso, D., Arias, C. A., et al. (2010). The fms21 (pilA)-fms20 Locus Encoding One of Four Distinct Pili of Enterococcus Faecium is Harboured on a Large Transferable Plasmid Associated With Gut Colonization and Virulence. J. Med. Microbiol. 59 (Pt 4), 505-507. doi: 10.1099/jmm.0.016238-0

Kirienko, N. V., Ausubel, F. M., and Ruvkun, G. (2015). Mitophagy Confers Resistance to Siderophore-Mediated Killing by Pseudomonas Aeruginosa. Proc. Natl. Acad. Sci. U. S. A. 112 (6), 1821-1826. doi: 10.1073/pnas.1424954112

Kirienko, N. V., Cezairliyan, B. O., Ausubel, F. M., and Powell, J. R. (2014). Pseudomonas Aeruginosa PA14 Pathogenesis in Caenorhabditis Elegans. Methods Mol. Biol. 1149, 653-669. doi: 10.1007/978-1-4939-0473-0_50

Kirienko, N. V., Kirienko, D. R., Larkins-Ford, J., Wählby, C., Ruvkun, G., and Ausubel, F. M. (2013). Pseudomonas Aeruginosa Disrupts Caenorhabditis Elegans Iron Homeostasis, Causing a Hypoxic Response and Death. Cell Host Microbe 13 (4), 406-416. doi: 10.1016/j.chom.2013.03.003

Kurz, C., Chauvet, S., Andrès, E., Aurouze, M., Vallet, I., Michel, G., et al. (2003). Virulence Factors of the Human Opportunistic Pathogen Serratia Marcescens Identified by In Vivo Screening. EMBO J. 22 (7), 1451-1460. doi: 10.1093/ emboj/cdg159

Lebreton, F., Valentino, M., Schaufler, K., Earl, A., Cattoir, V., and Gilmore, M. (2018). Transferable Vancomycin Resistance in Clade B Commensal-Type Enterococcus Faecium. J. Antimicrob. Chemother. 73 (6), 1479-1486. doi: 10.1093/jac/dky039

Lebreton, F., van Schaik, W., McGuire, A., Godfrey, P., Griggs, A., Mazumdar, V., et al. (2013). Emergence of Epidemic Multidrug-Resistant Enterococcus Faecium From Animal and Commensal Strains. mBio 4 (4), e00534-e00513. doi: $10.1128 / \mathrm{mBio} .00534-13$

Levitus, M., Rewane, A., and Perera, T. B. (2020) Vancomycin-Resistant Enterococci: Statpearls. Available at: https://www.ncbi.nlm.nih.gov/books/ NBK513233/ (Accessed Accessed: 2020/07/21).

Liu, Q., Ponnuraj, K., Xu, Y., Ganesh, V. K., Sillanpaa, J., Murray, B. E., et al. (2007). The Enterococcus Faecalis MSCRAMM ACE Binds its Ligand by the Collagen Hug Model. J. Biol. Chem. 282 (27), 19629-19637. doi: 10.1074/ jbc.M611137200

Montealegre, M., Singh, K., Somarajan, S., Yadav, P., Chang, C., Spencer, R., et al. (2016). 'Role of the Emp Pilus Subunits of Enterococcus Faecium in Biofilm Formation, Adherence to Host Extracellular Matrix Components, and
Experimental Infection'. Infect. Immun. 84 (5), 1491-1500. doi: 10.1128/ IAI.01396-15

Moy, T. I., Conery, A. L., Larkins-Ford, J., Wu, G., Mazitschek, R., Casadei, G., et al. (2009). High-Throughput Screen for Novel Antimicrobials Using a Whole Animal Infection Model. ACS Chem. Biol. 4 (7), 527-533. doi: $10.1021 / \mathrm{cb} 900084 \mathrm{v}$

Moy, T., Mylonakis, E., Calderwood, S., and Ausubel, F. (2004). 'Cytotoxicity of Hydrogen Peroxide Produced by Enterococcus Faecium'. Infect. Immun. 72 (8), 4512-4520. doi: 10.1128/IAI.72.8.4512-4520.2004

Mundy, L., Sahm, D., and Gilmore, M. (2000). Relationships Between Enterococcal Virulence and Antimicrobial Resistance. Clin. Microbiol. Rev. 13 (4), 513-522. doi: 10.1128/CMR.13.4.513

Murray, B. (2000). 'Vancomycin-Resistant Enterococcal Infections. New Engl. J. Med. 342 (10), 710-721. doi: 10.1056/NEJM200003093421007

Nallapareddy, S. R., Singh, K. V., and Murray, B. E. (2006). Construction of Improved Temperature-Sensitive and Mobilizable Vectors and Their Use for Constructing Mutations in the Adhesin-Encoding Acm Gene of Poorly Transformable Clinical Enterococcus Faecium Strains. Appl. Environ. Microbiol. 72 (1), 334-345. doi: 10.1128/AEM.72.1.334-345.2006

Nallapareddy, S. R., Singh, K. V., and Murray, B. E. (2008). Contribution of the Collagen Adhesin Acm to Pathogenesis of Enterococcus Faecium in Experimental Endocarditis. Infect. Immun. 76 (9), 4120-4128. doi: 10.1128/IAI.00376-08

Nallapareddy, S. R., Weinstock, G. M., and Murray, B. E. (2003). Clinical Isolates of Enterococcus Faecium Exhibit Strain-Specific Collagen Binding Mediated by Acm, a New Member of the MSCRAMM Family. Mol. Microbiol. 47 (6), 17331747. doi: 10.1046/j.1365-2958.2003.03417.x

Neeraja, M., Lakshmi, V., Padmasri, C., and Padmaja, K. (2017). Utility of Acridine Orange Staining for Detection of Bacteria From Positive Blood Cultures. J. Microbiol. Methods 139, 215-217. doi: 10.1016/ j.mimet.2017.06.014

Palmer, K., Godfrey, P., Griggs, A., Kos, V., Zucker, J., Desjardins, C., et al. (2012). Comparative Genomics of Enterococci: Variation in Enterococcus Faecalis, Clade Structure in E. Faecium, and Defining Characteristics of E. Gallinarum and E. Casseliflavus. mBio 3 (1), e00318-e00311. doi: 10.1128/mBio.00318-11

Panesso, D., Montealegre, M. C., Rincon, S., Mojica, M. F., Rice, L. B., Singh, K. V., et al. (2011). The hylEfm Gene in pHylEfm of Enterococcus Faecium is Not Required in Pathogenesis of Murine Peritonitis. BMC Microbiol. 11 (1), 20. doi: 10.1186/1471-2180-11-20

Pukkila-Worley, R., and Ausubel, F. (2012). Immune Defense Mechanisms in the Caenorhabditis Elegans Intestinal Epithelium. Curr. Opin. Immunol. 24 (1), 3-9. doi: 10.1016/j.coi.2011.10.004

Pukkila-Worley, R., Feinbaum, R., Kirienko, N. V., Larkins-Ford, J., Conery, A. L., and Ausubel, F. M. (2012). Stimulation of Host Immune Defenses by a Small Molecule Protects C. Elegans From Bacterial Infection. PloS Genet. 8 (6), e1002733. doi: 10.1371/journal.pgen.1002733

Qin, X., Galloway-Peña, J., Sillanpaa, J., Roh, J., Nallapareddy, S., Chowdhury, S., et al. (2012). Complete Genome Sequence of Enterococcus Faecium Strain TX16 and Comparative Genomic Analysis of Enterococcus Faecium Genomes. BMC Microbiol. 12, 135. doi: 10.1186/1471-2180-12-135

Ramos-Martinez, A., Fernandez-Cruz, A., Dominguez, F., Forteza, A., Cobo, M., Sanchez-Romero, I., et al. (2020). Hospital-Acquired Infective Endocarditis During Covid-19 Pandemic. Infect. Prev. Pract. 2 (3), 100080. doi: 10.1016/ j.infpip.2020.100080

Raven, K., Reuter, S., Reynolds, R., Brodrick, H., Russell, J., Török, M., et al. (2016). A Decade of Genomic History for Healthcare-Associated Enterococcus Faecium in the United Kingdom and Ireland. Genome Res. 26 (10), 13881396. doi: $10.1101 /$ gr.204024.116

Rosiana, S., Zhang, L., Kim, G. H., Revtovich, A. V., Uthayakumar, D., Sukumaran, A., et al. (2021). Comprehensive Genetic Analysis of Adhesin Proteins and Their Role in Virulence of Candida Albicans. Genetics 217 (2), iyab003. doi: 10.1093/genetics/iyab003

Shivers, R. P., Pagano, D. J., Kooistra, T., Richardson, C. E., Reddy, K. C., Whitney, J. K., et al. (2010). Phosphorylation of the Conserved Transcription Factor ATF-7 by PMK-1 P38 MAPK Regulates Innate Immunity in Caenorhabditis Elegans. PloS Genet. 6 (4), e1000892. doi: 10.1371/journal.pgen.1000892

Sillanpää, J., Nallapareddy, S., Prakash, V., Qin, X., Höök, M., Weinstock, G., et al. (2008). Identification and Phenotypic Characterization of a Second Collagen Adhesin, Scm, and Genome-Based Identification and Analysis of 13 Other 
Predicted MSCRAMMs, Including Four Distinct Pilus Loci, in Enterococcus Faecium. Microbiol. (Reading England) 154 (Pt 10), 3199-3211. doi: 10.1099/ mic.0.2008/017319-0

Sillanpää, J., Nallapareddy, S., Singh, K., Prakash, V., Fothergill, T., Ton-That, H., et al. (2010). Characterization of the $E b p(F m)$ Pilus-Encoding Operon of Enterococcus Faecium and its Role in Biofilm Formation and Virulence in a Murine Model of Urinary Tract Infection. Virulence 1 (4), 236-246. doi: 10.4161/viru.1.4.11966

Sillanpää, J., Prakash, V., Nallapareddy, S., and Murray, B. (2009). Distribution of Genes Encoding MSCRAMMs and Pili in Clinical and Natural Populations of Enterococcus Faecium. J. Clin. Microbiol. 47 (4), 896-901. doi: 10.1128/JCM.02283-08

Singh, K. V., Qin, X., Weinstock, G. M., and Murray, B. E. (1998). Generation and Testing of Mutants of Enterococcus Faecalis in a Mouse Peritonitis Model. J. Infect. Dis. 178 (5), 1416-1420. doi: 10.1086/314453

Somarajan, S., Roh, J., Singh, K., Weinstock, G., and Murray, B. (2014). Ccpa is Important for Growth and Virulence of Enterococcus Faecium. Infect. Immun. 82 (9), 3580-3587. doi: 10.1128/IAI.01911-14

Stiernagle, T. (2006). Maintenance of C. Elegans'. Wormbook. The C. elegans Research Community. p.1-11. doi: 10.1895/wormbook.1.101.1

Tan, M. W., Mahajan-Miklos, S., and Ausubel, F. M. (1999). Killing of Caenorhabditis Elegans by Pseudomonas Aeruginosa Used to Model Mammalian Bacterial Pathogenesis. Proc. Natl. Acad. Sci. U. S. A. 96 (2), 715-720. doi: 10.1073/pnas.96.2.715

Tjahjono, E., and Kirienko, N. V. (2017). A Conserved Mitochondrial Surveillance Pathway is Required for Defense Against Pseudomonas Aeruginosa. PloS Genet. 13 (6), e1006876. doi: 10.1371/journal.pgen.1006876
Tran, T. T., Panesso, D., Gao, H., Roh, J. H., Munita, J. M., Reyes, J., et al. (2013) Whole-Genome Analysis of a Daptomycin-Susceptible Enterococcus Faecium Strain and its Daptomycin-Resistant Variant Arising During Therapy. Antimicrob. Agents Chemother. 57 (1), 261-268. doi: 10.1128/AAC.01454-12

Treitman, A., Yarnold, P., Warren, J., and Noskin, G. (2005). Emerging Incidence of Enterococcus Faecium Among Hospital Isolates, (1993 to 2002). J. Clin. Microbiol. 43 (1), 462-463. doi: 10.1128/JCM.43.1.462-463.2005

Willems, R., Homan, W., Top, J., van Santen-Verheuvel, M., Tribe, D., Manzioros, X., et al. (2001). Variant Esp Gene as a Marker of a Distinct Genetic Lineage of Vancomycin-Resistant Enterococcus Faecium Spreading in Hospitals. Lancet (London England) 357 (9259), 853-855. doi: 10.1016/S0140-6736(00)04205-7

Yuen, G., and Ausubel, F. (2018). Both Live and Dead Enterococci Activate Caenorhabditis Elegans Host Defense Via Immune and Stress Pathways. Virulence 9 (1), 683-699. doi: 10.1080/21505594.2018.1438025

Conflict of Interest: The authors declare that the research was conducted in the absence of any commercial or financial relationships that could be construed as a potential conflict of interest.

Copyright (C) 2021 Revtovich, Tjahjono, Singh, Hanson, Murray and Kirienko. This is an open-access article distributed under the terms of the Creative Commons Attribution License (CC BY). The use, distribution or reproduction in other forums is permitted, provided the original author(s) and the copyright owner(s) are credited and that the original publication in this journal is cited, in accordance with accepted academic practice. No use, distribution or reproduction is permitted which does not comply with these terms. 


\section{OPEN ACCESS}

Edited by:

Buket Baddal,

Boston Children's Hospital and

Harvard Medical School, United States

Reviewed by:

Rajagopal Kammara

Central Food Technological Research

Institute (CSIR), India

Shinsmon Jose

University of Cincinnati, United States

*Correspondence:

Lu-Quan L

liluquan123@163.com

Zheng-Li Wang

zhengli_wang@126.com

${ }^{\dagger}$ These authors have contributed equally to this work and

share first authorship

Specialty section:

This article was submitted to

Bacteria and Host,

a section of the journal

Frontiers in Cellular and

Infection Microbiology

Received: 13 April 2021

Accepted: 21 July 2021

Published: 05 August 2021

Citation:

Ji Y-C, Sun Q, Fu C-Y, She X, Liu X-C, He Y, Ai Q, Li L-Q and Wang Z-L (2021) Exogenous Autoinducer-2

Rescues Intestinal Dysbiosis and Intestinal Inflammation in a Neonatal Mouse Necrotizing Enterocolitis Model. Front. Cell. Infect. Microbiol. 11:694395.

doi: 10.3389/fcimb.2021.694395

\section{Exogenous Autoinducer-2 Rescues Intestinal Dysbiosis and Intestinal Inflammation in a Neonatal Mouse Necrotizing Enterocolitis Model}

\author{
Yan-Chun Ji ${ }^{1,2,3,4,5 \dagger}$, Qian Sun ${ }^{1,2,3,4,5 \dagger}$, Chun-Yan Fu ${ }^{1,2,3,4,5 \dagger}$, Xiang She $e^{1,2,3,4,5}$, \\ Xiao-Chen Liu ${ }^{1,2,3,4,5}$, Yu He $\mathrm{H}^{1,2,3,4,5}$, Qing Ai ${ }^{1,2,3,4,5}$, Lu-Quan $\mathrm{Li}^{1,2,3,4,5^{*}}$ \\ and Zheng-Li Wang 1,2,3,4,5*
}

\begin{abstract}
${ }^{1}$ Neonatal Diagnosis and Treatment Center of Children's Hospital of Chongqing Medical University, Chongqing, China, 2 National Clinical Research Center for Child Health and Disorders, Chongqing, China, ${ }^{3}$ Ministry of Education Key Laboratory of Child Development and Disorders, Chongaing, China, ${ }^{4}$ China International Science and Technology Cooperation Base of Child Development and Critical Disorders, Chongqing, China, ${ }^{5}$ Chongqing Key Laboratory of Pediatrics, Chongqing, China
\end{abstract}

Autoinducer-2 (Al-2) is believed to be a bacterial interspecies signaling molecule that plays an important role in the regulation of the physiological behaviors of bacteria. The effect of Al-2 on the process of necrotizing enterocolitis (NEC) is unknown, and the aim of this study was to study the effect of Al-2 in a mouse NEC model. C57BL/6 mouse pups were randomly divided into three groups: the control group, the NEC group, and the NEC+Al-2 (NA) group. Exogenous Al-2 (500 nM) was added to the formula milk of the NA group. The concentrations of fecal Al-2 and flora were tested. The expression of cytokines, TLR4 and $\mathrm{NF}-\kappa \mathrm{B}$ in intestinal tissue was detected. The Al-2 level was significantly decreased in the NEC group $(P<0.05)$. Compared with the NEC group, the intestinal injury scores, expression of TLR4, NF-kB, and proinflammatory factors (IL-1 $\beta, I L-6, I L-8$ and TNF- $\alpha$ ) were reduced, and expression of anti-inflammatory factor (IL-10) was increased in the NA group mice $(P<0.05)$. At the phylum level, the Proteobacteria abundance in the NA group was significantly increased, while the Bacteroidota abundance in the control group was significantly increased $(P<0.05)$. At the genus level, Helicobacter and Clostridium_sensu_stricto_1 exhibited significantly greater abundance in the NEC group than in the other two groups, while Lactobacillus had the opposite trend $(P<0.05)$. In addition, the abundances of Klebsiella, Rodentibacter and Enterococcus were significantly higher in the NA group than in the NEC and control groups $(P<0.05)$. Exogenous Al-2 partially reverses flora disorder and decreases inflammation in an NEC mouse model.

Keywords: necrotizing enterocolitis (NEC), autoinducer-2 (Al-2), intestinal flora, cytokines, intestinal immunity 


\section{INTRODUCTION}

Necrotizing enterocolitis (NEC) is a devastating intestinal disease that is a significant cause of death in neonatal intensive care units (Frost et al., 2017; Isani et al., 2018). Five to twelve percent of very low-birth-weight infants were affected by NEC, and surgical intervention was needed in $20-40 \%$ of cases (Shulhan et al., 2017). The pathogenesis of NEC is complicated and multifactorial (Niño et al., 2016; Bellodas Sanchez and Kadrofske, 2019), and abnormal microbial colonization and a strong immune response in the gut may be responsible (Neu and Walker, 2011; Raveh-Sadka et al., 2015). The intestinal flora can not only regulate an inflammatory reaction of the intestinal wall through toxic factors but also affect the proliferation, differentiation and gene expression of intestinal wall cells (Hinde and Lewis, 2015). Imbalance in the intestinal flora might disrupt the immune balance of the body, destroy intestinal barrier function, and cause excessive inflammation in intestinal wall tissue, resulting in local or diffuse necrosis (Egan et al., 2016; Shi et al., 2017; Baranowski and Claud, 2019).

Quorum sensing (QS), a microbial cell communication process, dynamically controls different metabolic and physiological activities (Wu et al., 2020). Autoinducer-2 (AI-2), a small molecule, is involved in the QS system, and its production and reaction have been observed throughout the bacterial kingdom (Pereira et al., 2013; Wu et al., 2020). AI-2 is synthesized by many bacteria via enzymatic steps. The AI-2 precursor (S)-4,5-dihydroxypentane-2,3-dione (DPD) can be synthesized via catalytic reactions and spontaneously cyclizes into AI-2 (De Keersmaecker et al., 2005). AI-2 signaling can promote interspecies communication and allow different bacterial species to alter behaviors such as biofilm formation, luminescence and virulence (Cuadra-Saenz et al., 2012; Thompson et al., 2015). AI-2 plays an important role in the colonization of the intestinal flora (Buck et al., 2009). Artificially increasing the level of AI-2 can attenuate antibiotic-induced intestinal dysbiosis, which plays an important role in the development of NEC (Sun et al., 2015). We have previously reported that the AI-2 concentration decreases significantly in the acute phase of NEC and increases gradually in the recovery phase of NEC (Fu et al., 2020). These findings illustrate that AI-2 may be an important target for the correction of intestinal flora imbalance.

Toll-like receptors (TLRs), the classic pattern recognition receptors (PRRs) of the human gut, are able to specifically recognize microbial-associated molecular patterns to induce innate and adaptive immune responses (Kawai and Akira, 2010). An imbalance of the flora may activate TLR4, a major lipopolysaccharide (LPS) receptor in TLRs, and then activate the downstream mediator nuclear factor kappa $(\mathrm{NF}-\kappa \mathrm{K})$, further inducing the transcription of various proinflammatory and anti-inflammatory cytokines (Park and Lee, 2013; Mihi and Good, 2019; Van Belkum et al., 2020). TLR4 signaling is an important inflammatory pathway related to bacteria in the development of NEC (Hackam and Sodhi, 2018).

On this background, we hypothesized that AI- 2 can regulate the composition of the intestinal flora, correct imbalance of the intestinal flora, and reduce the degree of inflammation in the context of NEC by inhibiting the TLR4/NF- $\mathrm{KB}$ signaling pathway. To test this hypothesis, we established an NEC animal model and gavaged the model animals with AI-2 to clarify the influence of AI-2 on the intestinal flora composition and the NEC disease process.

\section{MATERIALS AND METHODS}

\section{Induction of Neonatal NEC Mouse Model}

The NEC mouse model was constructed according to the methods in previous studies, with some adjustments (Garg et al., 2015; Xiao et al., 2018; Gopalakrishna et al., 2019). Seven-day-old C57BL/6 mouse pups were randomly divided into three groups. The mice in the control group were allowed to nurse their mothers freely; those in the experimental group were fed formula milk ( $2 \mathrm{~g}$ of Similac Advance in $10 \mathrm{ml}$ of 33\% Esbilac Puppy Milk Replacer); and those in the intervention group were fed AI-2-containing formula milk (2 $\mathrm{g}$ of Similac Advance with $1 \mathrm{ml}$ of $5 \mu \mathrm{M}$ AI-2 in $9 \mathrm{ml}$ of 33\% Esbilac Puppy Milk Replacer), with a final concentration of AI-2 in the formula milk of $500 \mathrm{nM}$, administered at $30 \mu \mathrm{l} / \mathrm{g}$ body weight via a silicone tube (1.9 Fr) every $4 \mathrm{~h}$ for 3 days. After the pups in the experimental group and the intervention group were fed the Esbilac formula, they were subjected to asphyxia (100\% N2 for $90 \mathrm{~s})$ and cold stress at $4^{\circ} \mathrm{C}(10 \mathrm{~min})$ three times a day for 3 days, while the control pups were not subjected to cold stress or asphyxia. The mice were put back to the cage for relaxation after each feeding or hypoxia-cold stimulation during the modeling period. Three-day NEC modeling was completed after the last feeding. The mice were left to relax for $12 \mathrm{~h}$ after feeding, and then intestinal tissues and contents were harvested as test samples. The animal study was reviewed and approved by the Institutional Animal Care and Use Committee at Chongqing Medical University.

\section{Histology}

Three groups of pups were decapitated after NEC induction was completed. The intestine was taken from the body of each mouse, and a $1-\mathrm{cm}$ distal part of the ileum was fixed in paraformaldehyde solution (4\%). Then, the sample was dehydrated and embedded in paraffin. Next, the sample was cut into $4-\mu \mathrm{m}$ slices. Subsequently, the $4-\mu \mathrm{m}$ tissue slices were stained with hematoxylin and eosin (HE). The degree of NEC was graded by a blinded evaluator using the following standard histological scoring system (Yu et al., 2009): 0, no damage; 1, epithelial cell lifting or separation; 2, moderate villus necrosis; 3 , necrosis of the entire villus; and 4 , transmural necrosis.

\section{Immunohistochemistry}

Paraffin sections were deparaffinized in xylene and rehydrated with ethanol. The sections were used for immunohistochemistry. The tissue sections were placed in a repair box filled with citric acid $(\mathrm{pH}$ 6.0) antigen retrieval buffer and heated for antigen retrieval in a microwave oven for $25 \mathrm{~min}$. Then, the sections were 
incubated in 3\% hydrogen peroxide at room temperature in the dark for 25 min and washed with PBS ( $\mathrm{pH} 7.4$ ). BSA (3\%) was added to the sections to evenly cover the tissue, and the tissues were sealed for $30 \mathrm{~min}$. The sealing solution was gently removed, the primary antibody prepared with PBS ( $\mathrm{pH} 7.4$ ) was added to the sections, and the sections were placed flat in a box and incubated overnight at $4^{\circ} \mathrm{C}$. The sections were then placed in PBS ( $\mathrm{pH}$ 7.4) and washed three times. The tissues were covered with an anti-rabbit antibody (HRP-labeled) and incubated at room temperature for $50 \mathrm{~min}$. A chromogenic reaction was performed with $\mathrm{DAB}$ color developing solution. The sections were counterstained with hematoxylin stain solution. Staining of the tissues was detected with an optical microscope. Nuclear staining with hematoxylin was blue, and positive staining for DAB was brownish yellow.

\section{Mouse Intestinal Content Acquisition and Al-2 Activity Measurement}

The methods have been previously described (Fu et al., 2020). The ileum and colon of each mouse were irrigated with $400 \mu \mathrm{l}$ of $2216 \mathrm{E}$ liquid medium, and the contents were gathered with sterile tubes, vortexed and centrifuged. The supernatants were filtered through a filter membrane (Millipore, USA), and the filtrates were collected as samples to be tested. The fecal sediment was frozen at $-80^{\circ} \mathrm{C}$ for detection of intestinal flora. A chemically synthesized AI-2 precursor DPD was purchased from Omm Scientific (Dallas, TX, USA). The AI-2 activity in the samples was detected using the Vibrio harveyi reporter strain BB170 (Raut et al., 2013). The BB170 strain was grown in 2216E (QDRS BIOTEC, China) liquid medium $\left(30^{\circ} \mathrm{C}\right.$ for $\left.18 \mathrm{~h}\right)$ and diluted 1:5,000 into fresh $2216 \mathrm{E}$ liquid medium. The samples were added to V. harveyi BB170 strain diluent for the AI-2 assay. Additionally, $20 \mu \mathrm{l}$ of $1 \mu \mathrm{M}$ AI-2 (Omm Scientific, USA) standard solution, fecal filtrate, and 2216E liquid medium (as a negative control) were added in quintuplicate to a 96-well assay plate (Corning, USA). Then, $180 \mu \mathrm{l}$ of BB170 diluent was added to a 96-well assay plate to produce a final volume of $200 \mu \mathrm{l}$, and the plate was shaken at $30^{\circ} \mathrm{C}$ and $120 \mathrm{rpm}$. After $30 \mathrm{~min}$, a BioTek Synergy H1 (USA) instrument was used to measure the bioluminescence intensity every $0.5 \mathrm{~h}$ until the value of the negative control group was minimized.

\section{Fecal Sample Microbiota Analysis}

Fecal microbiota genomic DNA was extracted with a QIAamp FAST DNA Stool Mini-Kit (Qiagen, Germany) according to the manufacturer's instructions, as previously described (Fu et al., 2020). The DNA extracts were detected on $1 \%$ agarose gels, and the DNA concentration and purity were assessed with a spectrophotometer (NanoDrop 2000 UV-vis, Thermo Scientific, USA). The V3-V4 hypervariable region of the intestinal bacterial 16S rDNA gene was amplified with the primers 338F (5'-ACTCCTACGGGAGGCAGCAG-3') and 806R (5'-GGACTACHVGGGTWTCTAAT - 3'). The amplification procedure was as follows: initial denaturation $\left(95^{\circ} \mathrm{C}\right.$ for $\left.3 \mathrm{~min}\right)$; denaturation $\left(95^{\circ} \mathrm{C}\right.$ for $\left.30 \mathrm{~s}\right)$, annealing $\left(55^{\circ} \mathrm{C}\right.$ for $30 \mathrm{~s})$ and extension $\left(72^{\circ} \mathrm{C}\right.$ for $\left.45 \mathrm{~s}\right)$ for a total of 27 cycles; and an additional extension $\left(72^{\circ} \mathrm{C}\right.$ for $\left.10 \mathrm{~min}\right)$. The product was separated by $2 \%$ agarose gel electrophoresis, recovered using an AxyPrep DNA Gel Extraction Kit (Axygen Biosciences) and quantified with a Quantus ${ }^{\mathrm{TM}}$ Fluorometer (Promega, USA). A database was created and sequenced on an Illumina MiSeqrelated platform. The raw data were processed. Briefly, bases with a quality score $<20$ were truncated, and sequences with lengths $>10$ bp overlapped. Reads that exceeded the maximum mismatch ratio of 0.2 in the overlapping region of the splicing sequence were removed. The reads were distinguished according to the primers and barcode, and the sequence direction was adjusted to ensure exact barcode matching. The processed sequences were divided into operational taxonomic units (OTUs) using UPARSE (version 7.1) and OTU clustering of the sequences with a $97 \%$ similarity threshold.

\section{Quantitative Real-Time PCR (qRT-PCR)}

RNA was extracted from the ilea of mice and subjected to reverse transcription (RT) with a kit from Takara (Takara, Japan). The resulting cDNA was used for qRT-PCR assays with a TB Green Premix Ex Taq II (Tli RNase H Plus) Kit (Takara, Japan). Glyceraldehyde-3-phosphate dehydrogenase (GAPDH) was used to normalize the input mRNA levels (TLR4, NF- $\mathrm{KB}$, IL$1 \beta$, IL-6, IL-8, IL-10, and TNF- $\alpha$ ) as an endogenous housekeeping gene. The following primer sequences were used (Table 1). The results are shown as the mean $2^{-\Delta \Delta C t} \pm \mathrm{SD}$.

\section{Enzyme-Linked Immunosorbent Assay (ELISA)}

The levels of the inflammatory cytokines IL-1 $\beta$, IL-6, IL-8, tumor necrosis factor- $\alpha$ (TNF- $\alpha$ ) and IL-10 were investigated in the supernatant of mouse intestinal tissue with mouse IL-1 $\beta$, IL-6, TNF- $\alpha$ and IL-10 ELISA kits (4A BIOTECH, China), respectively, according to the manufacturer's instructions.

\section{Western Blotting (WB)}

Intestinal tissues were homogenized with an electric homogenizer in NP-40 lysis buffer (Beyotime, China). The concentrations of

TABLE 1 | List of primers

\begin{tabular}{|c|c|c|}
\hline Gene & Direction & Primers \\
\hline \multirow[t]{2}{*}{ GAPDH } & Forward & TGAAGCAGGCATCTGAGGG \\
\hline & Reverse & CGAAGGTGGAAGAGTGGGAG \\
\hline \multirow[t]{2}{*}{ TLR4 } & Forward & TाTATCAGAGCCGTTGGTG \\
\hline & Reverse & CAGAGGATTGTCCTCCCATT \\
\hline \multirow[t]{2}{*}{$N F-\kappa B$} & Forward & ATGTGCATCGGCAAGTGG \\
\hline & Reverse & CAGAAGTTGAGTITCGGGTAG \\
\hline \multirow[t]{2}{*}{$I L-1 \beta$} & Forward & TGGTGTGTGACGTTCCCATT \\
\hline & Reverse & CAGCACGAGGCTIIITGTTG \\
\hline \multirow[t]{2}{*}{ IL-6 } & Forward & CCAAGAGGTGAGTGCTTCCC \\
\hline & Reverse & CTGTTGTTCAGACTCTCTCCCT \\
\hline \multirow[t]{2}{*}{ IL-8 } & Forward & CAAGGCTGGTCCATGCTCC \\
\hline & Reverse & TGCTATCACTTCCTITCTGTTGC \\
\hline \multirow[t]{2}{*}{ IL-10 } & Forward & GCCGTCATITTCTGCCTCAT \\
\hline & Reverse & GCTTCCCTATGGCCCTCATT \\
\hline \multirow[t]{2}{*}{$\mathrm{TNF}-\alpha$} & Forward & CCAAAGGGATGAGAAGTTCC \\
\hline & Reverse & CTCCACTTGGTGGTTTGCTA \\
\hline
\end{tabular}


extracted proteins were measured using a bicinchoninic acid assay kit (Beyotime). The protein solution was mixed with sodium lauryl sulfate sample buffer (Beyotime) at a ratio of 4:1 and then denatured in boiling water for $5 \mathrm{~min}$. The protein samples were separated in a $10 \%$ polyacrylamide gel and then transferred onto a polyvinylidene difluoride membrane. The membranes were blocked at room temperature in quick sealing fluid for $10 \mathrm{~min}$ and then incubated with antibodies against TLR4, NF- $\kappa B$ and $\beta$-actin at $4^{\circ} \mathrm{C}$ overnight. Subsequently, the membranes were washed and incubated with an anti-rabbit secondary antibody for $2 \mathrm{~h}$. The protein bands were detected using an enhanced chemiluminescence kit (ZENBIO Biotechnology, China) and visualized using a Bio-Rad ChemiDoc $^{\mathrm{TM}}$ Touch imaging system. The images were analyzed using the Image Lab and Image J software.

\section{Statistical Analysis}

All data were analyzed using GraphPad Prism (Version 8.3.0) and tested for normal distribution. The mean \pm standard deviation (SD) was used to describe normally distributed data, and the differences were compared using one-way ANOVA or Student's t-test. The median and interquartile range (IQR) are used to describe skewed data, and the differences were analyzed by the Kruskal-Wallis test. $P<0.05$ was considered to indicate statistical significance.

\section{RESULTS}

\section{Al-2 Reduced the Severity in a Mouse NEC Model}

\section{Survival Rates}

During modeling, the control group exhibited no deaths, and none of the mice in the NEC group or NA group died before modeling. On the first day of modeling, three deaths occurred in the NEC group, and two deaths occurred in the NA group. On the second day of modeling, five deaths occurred in the NEC group, and three deaths occurred in the NA group. On the third day of modeling, five deaths occurred in the NEC group, and no deaths occurred in the NA group. A significant difference in the final survival rate among the three groups of pups was found: $90.74 \%(49 / 54)$ in the NA group, $75.93 \%(41 / 54)$ in the NEC group, and $100 \%(54 / 54)$ in the control group $(\chi 2=19.07$, $P=0.0001)$. The survival curves (Figure 1C) of the three groups were significantly different $(P<0.0001)$.

\section{General Condition and Weight Changes}

The neonatal mouse pups in the control group showed good growth and vitality and normal reactivity. The control mice had shiny fur and sufficient subcutaneous fat. The mice in the NEC

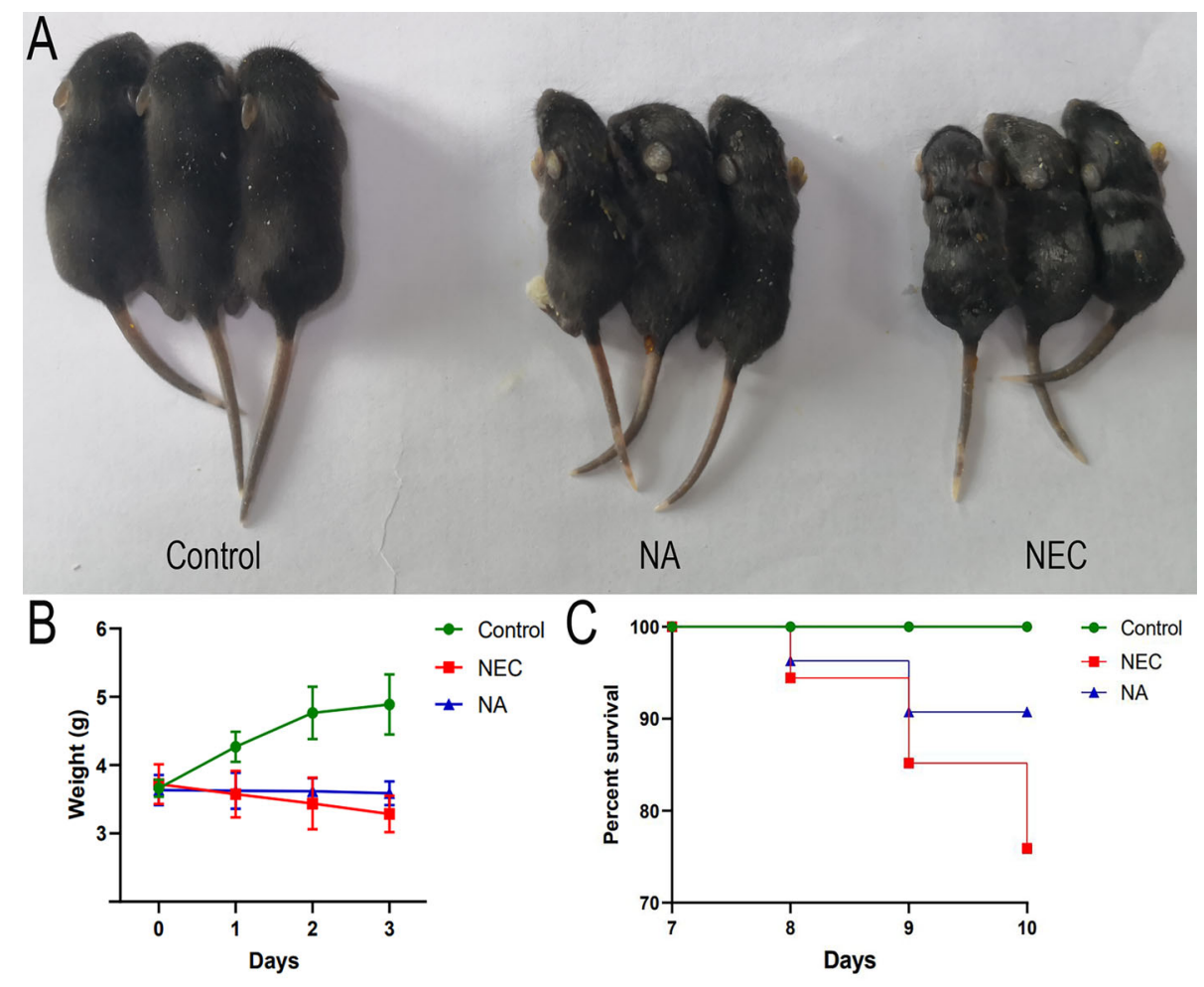

FIGURE 1 | (A) The growth of mice in the control group, mice in the NA group and mice in the NEC group. Mice in the NEC group were smaller than those in the control group and NA group. (B) Body weight changes of newborn mice in the three groups. Numbers of samples: Con ( $n=10)$, NEC ( $n=10)$, and NA ( $n=10)$. Statistics: two-way ANOVA multiple comparisons method. (C) Survival curves of newborn mice in three groups. The survival rate was estimated during modeling, and the results are shown as a Kaplan-Meier plot, with 54 mice in each group at the beginning. Statistics: log-rank (Mantel-Cox) test $(P<0.05)$. Control - normal control, NEC - necrotizing enterocolitis, NA - necrotizing enterocolitis+Al-2. 
group began to exhibit abdominal distension, bloating, diarrhea, black stool, and gradually decreased activity. The NA group mice exhibited no black stool and normal activity. The control group mice continued to gain weight during modeling. On days 1, 2, and 3 of NEC modeling, there were no significant differences in weight between the NEC group and the NA group. However, the body weights of the mice in the NEC group decreased during modeling, while the mice in the NA group remained basically unchanged during the modeling period (Figures 1A, B). Before sacrifice, the average weight of the mice in the NEC group was significantly lower than that of the mice in the NA group $(P=0.0157)$.

\section{Macroscopic Appearance of the Gut and Histological Scoring in Model Mice}

The guts of control mice had no macroscopic damage or pathological changes (Figure 2A). NEC mice exhibited obvious injury in the distal ileum, with intestinal gas distension, hemorrhage and discoloration (Figure 2B), while NA mice showed obvious gut gas distension and discoloration (Figure 2C).

Under an optical microscope, the intestinal tissue structure of the normal control group was clear and complete, with neatly arranged epithelial cells, a thick and continuous muscle layer and no obvious hyperemia, edema or separation in the mucosal layer, submucosa or lamina propria (Figures 2D, G). The intestinal tissue of the neonatal mice in the NEC group exhibited a disordered arrangement of epithelial cells, villous degeneration, edema, and partial necrosis, shedding or even disappearance of tissue. The muscle layer was obviously thinned or even broken, and edema was clearly observed in the mucosal layer, submucosa and lamina propria (Figures 2E, $\mathbf{H}$ ). In the NA group, mild to moderate edema, congestion, and villus edema were detected in the gut mucosa and submucosa (Figures 2F, I). HE staining of sections from NEC mice showed severe damage and tissue necrosis compared with those of control group and NA group mouse pups, the median intestinal histological score in NA group mice was significantly lower than that in NEC group mice (Figure 2J).

\section{Al-2 Partly Change the Intestinal Flora of NEC}

Figure 3 shows the AI-2 levels in the stool of newborn mice in the three groups. The average level in NEC mice was significantly lower than those in NA mice and control mice $(P<0.05)$. To investigate the effect of AI-2 on intestinal microbiota in NEC, the proportions of individual taxa in intestinal microbiota were analyzed, and the data are presented in Figure $\mathbf{4}$ and Supplementary Tables 1, 2. Figure 4 shows differences in intestinal microbiota among the three groups. At the phylum level (Figure 4A), the average relative abundance of Firmicutes was lower in the NEC group than in the NA and control groups $(P>0.05)$, while that of Campilobacterota was higher in NEC mice than in NA mice and control mice $(P<0.05)$. The average relative abundance of Proteobacteria was higher in NA mice than in NEC mice and control mice, while that of Bacteroidota was greater in control mice than in NEC mice and NA mice $(P<0.05)$.

At the genus level (Figure 4B), the average abundance of Lactobacillus was significantly lower in the NEC group than in the NA group and control group $(P<0.05)$. Clostridium_sensu_stricto_1 and Helicobacter were significantly more abundant in the NEC group than in the NA group and control group $(P<0.05)$. The abundances of Klebsiella, Enterococcus and Rodentibacter in the NA group were significantly greater than those in the NEC group and control group $(P<0.05)$. At the species level (Supplementary Figure 2), the average relative abundance of Clostridium_sensu_stricto_1 was higher in the NEC group than in the other two groups.

Furthermore, differences in the community composition were analyzed by weighted/unweighted UniFrac principal coordinates analysis (PCoA) to discriminate among the control, NEC and NA samples. There were overlaps among the three groups, as shown in Supplementary Figure 3.
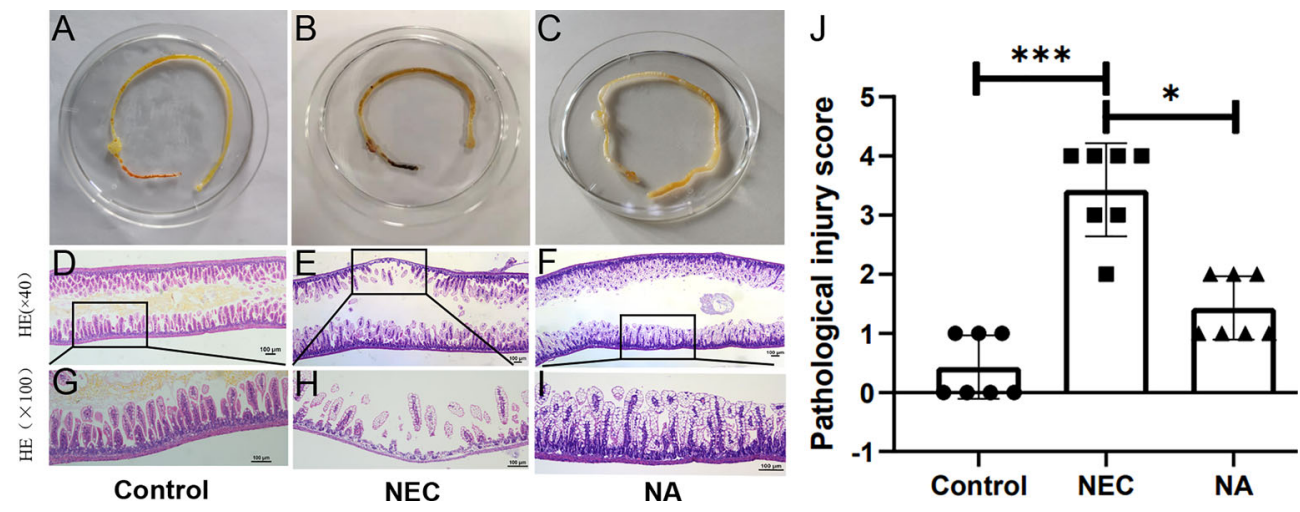

FIGURE 2 ( $\mathbf{A}-\mathbf{C})$ Macroscopic morphological analyses of the intestines of newborn mice in the three groups. (D-I) Images of HE staining by light microscopy. The histological damage in the terminal ilea in the three groups, control group (D, G), NEC group (E, H), NA group (F, I). Black rectangle indicates a representative area, with a zoomed-in image. Magnification: $\times 40, \times 100$. scale bar $=100 \mu \mathrm{m}$. (J) Gut histopathological injury scores in the control, NEC and NA group mice. Numbers of samples: Con $(n=7)$, NEC $(n=7)$, and NA ( $n=7)$. Statistics: Kruskal-Wallis test $\left({ }^{*} p<0.05 ;{ }^{* * *} p<0.001\right)$. Images of HE staining of each sample from the three groups are provided in Supplementary Figure 1. 


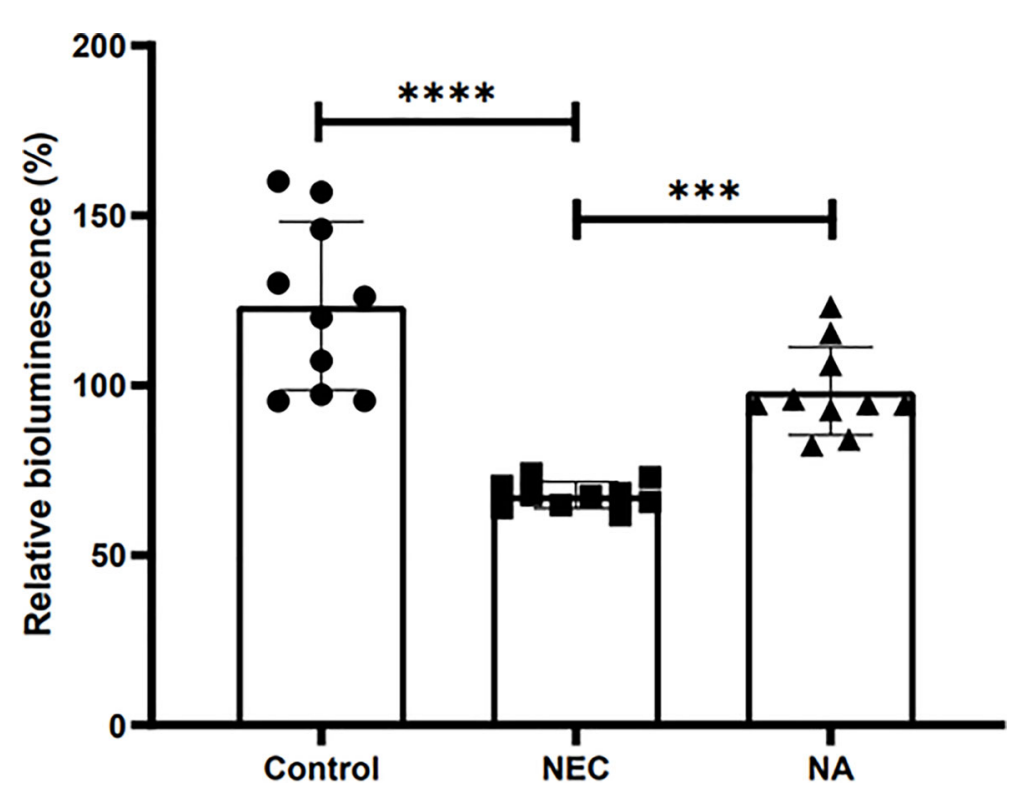

FIGURE 3 | The relative bioluminescence values in the mouse stools of $\mathrm{Al}-2$ in the three groups of newborn mice. Numbers of samples: Con ( $\mathrm{n}=10$ ), NEC ( $=10$ ), and NA $(n=10)$. Statistics: one-way ANOVA $\left(^{\star \star \star} p<0.001 ;{ }^{\star \star \star \star} \mathrm{P}<0.0001\right)$.

\section{Al-2 Reduced the Inflammatory Response}

To determine whether cytokine production in NEC mouse intestinal tissue was affected by AI-2, the expression of inflammatory cytokines was tested by qRT-PCR and ELISA. We found that the production of the inflammatory factors IL- $1 \beta$, IL-6, IL-8, TNF- $\alpha$ and IL-10 in NEC mice was twice, 2.98 times, 1.67 times, 1.88 times and 0.57 times that in NA mice, respectively, and the differences were statistically significant $(P<0.05)$. Meanwhile, the expression of proinflammatory factors, including IL-1 $\beta$ (Figure 5A), IL-6 (Figure 5B), IL-8 (Figure 5C) and TNF- $\alpha$ (Figure 5D), was significantly lower in NA mice than in NEC mice, and the production of the antiinflammatory cytokine IL-10 (Figure 5E) was significantly increased $(P<0.05)$.

TLR4, which plays a key role in the pathogenesis of NEC, is widely expressed in intestinal epithelial cells and various types of intestinal lymphocytes (Hackam and Sodhi, 2018). After TLR4 is activated by the corresponding pathogenic microorganism, it
A

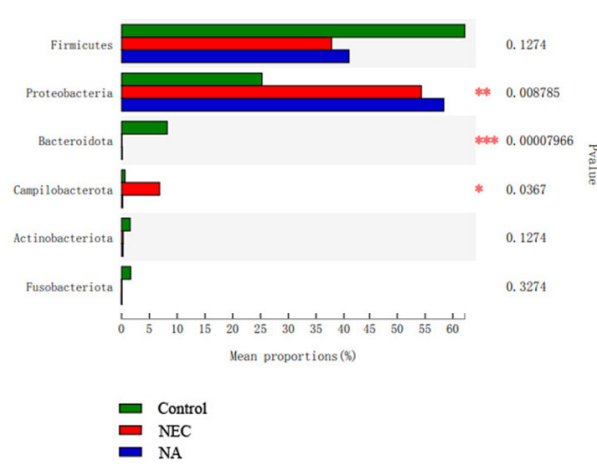

B

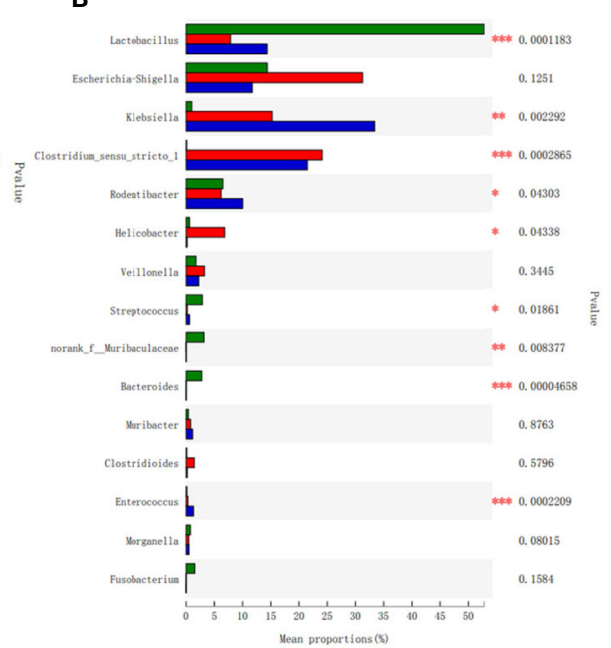

FIGURE 4 | (A) The flora composition of mice in the three groups at the phylum level. (B) The flora composition of mice in the three groups at the genus level. Numbers of samples: Con ( $n=12), N E C(n=10)$, and NA ( $n=11)$. Statistics: Kruskal-Wallis test with Scheffe's post-hoc test. ${ }^{\star} 0.01<P \leq 0.05,{ }^{\star \star} 0.001<P \leq 0.01,{ }^{\star \star \star} P \leq 0.001$. 

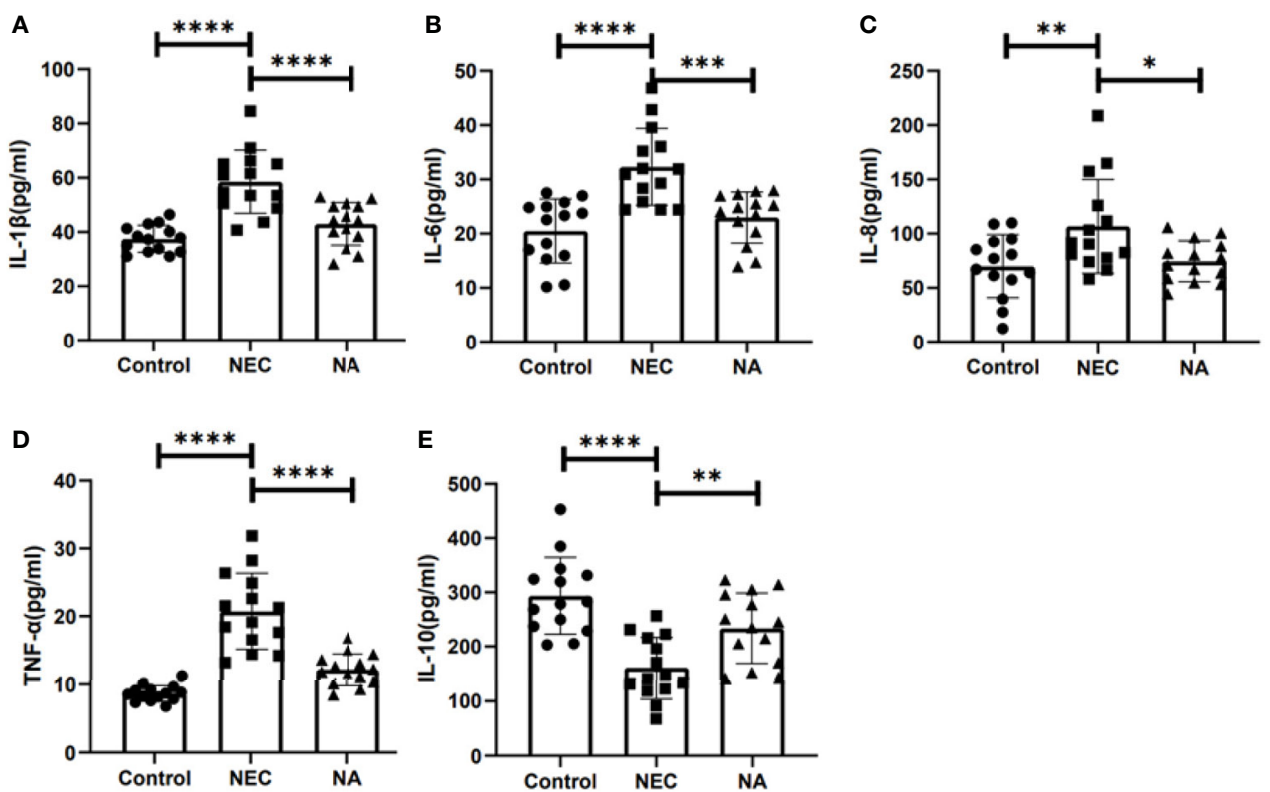

FIGURE 5 | The concentrations of inflammatory cytokines in the three groups were detected by ELISA. (A) The concentrations of IL-1 $\beta$. (B) The concentrations of IL-6. (C) The concentrations of IL-8. (D) The concentrations of TNF- $\alpha$. (E) The concentrations of IL-10. Numbers of samples: Con ( $n=14)$, NEC ( $n=14)$, and NA $(n=14)$. Statistics: one-way ANOVA $\left({ }^{*} \mathrm{p}<0.05 ;{ }^{* \star} \mathrm{p}<0.01 ;{ }^{* \star \star} \mathrm{p}<0.001 ;{ }^{* \star \star} \mathrm{p}<0.0001\right)$.

activates the innate immune response and further activates the downstream NF- $\mathrm{KB}$ signaling pathway and mediates the expression and release of the inflammatory factors IL-1, IL-6, IL-8 and TNF- $\alpha$ (Cohen and Prince, 2013). Based on the changes in the levels of inflammatory factors, we further tested the expression of TLR4 and NF- $\mathrm{KB}$ in NEC mouse intestinal tissues by qRT-PCR. We found that the transcript expression of TLR4 and NF-kB in NEC mice was 1.73 times and 2.10 times that in NA mice, respectively $(P<0.05)$. The TLR4 and NF- $\mathrm{KB}$ proteins were qualitatively analyzed by immunohistochemical staining, and we found that staining for both TLR4 (Figures 6A-C) and NF-KB (Figures 6D-F) was stronger in the NEC group than in the control and NA groups. Furthermore, we examined the protein expression levels of TLR4 and NF- $\mathrm{KB}$ in intestinal tissues by western blotting. Figure $\mathbf{6 G}$ shows the molecular weight of antibodies. The results showed that TLR4 (Figure 6H) and NF- $\mathrm{KB}$ (Figure 6I) expression in the NEC group was higher than that in the control. However, the expression of these proteins in the NEC group with the supplementation of exogenous $\mathrm{AI}-2$ was lower than that in the NEC group $(P<0.05)$.

\section{DISCUSSION}

An important finding of the current study was that exogenous AI-2 supplementation during the NEC modeling process reduced intestinal damage, significantly reduced the expression of TLR4 and related proinflammatory factors, and partially corrected the changes in flora in the NEC mouse model.

\section{The Relation Between NEC and Intestinal Flora Disorder}

Previous studies have shown that the intestinal microbiome is involved in NEC pathogenesis (Neu and Walker, 2011; RavehSadka et al., 2015). Dysbiosis of the intestinal flora existed in the NEC process (Niemarkt et al., 2015). At the phylum level, we found that the relative abundance of Proteobacteria was increased, while the relative abundance of Bacteroides was decreased in the NEC group. This finding was consistent with a meta-analysis of fecal microorganisms in NEC preterm infants (Pammi et al., 2017). At the genus level, we also found that the abundance levels of Clostridium_sensu_stricto_1 were significantly increased, and previous studies also found that Clostridium_sensu_stricto_1 is much more abundant in NEC infants (Fu et al., 2020). The proliferation of Clostridium species in the colon may produce toxins and lead to intestinal epithelial damage (Schönherr-Hellec and Aires, 2019). The abundance levels of Lactobacillus in the NEC group were significantly decreased in our study, and other studies indicated that administration of Lactobacillus is associated with a significantly decreased risk of NEC (Robertson et al., 2020). Based on these findings, intervention in intestinal flora disorders may be a potential mechanism for the treatment of NEC.

\section{Exogenous Al-2 Supplementation Partially Reversed the Changes in Intestinal Flora Dysbiosis}

AI-2 is an important regulatory factor, which responds to the fluctuations in the microbiota (Wang et al., 2019). Our previous 


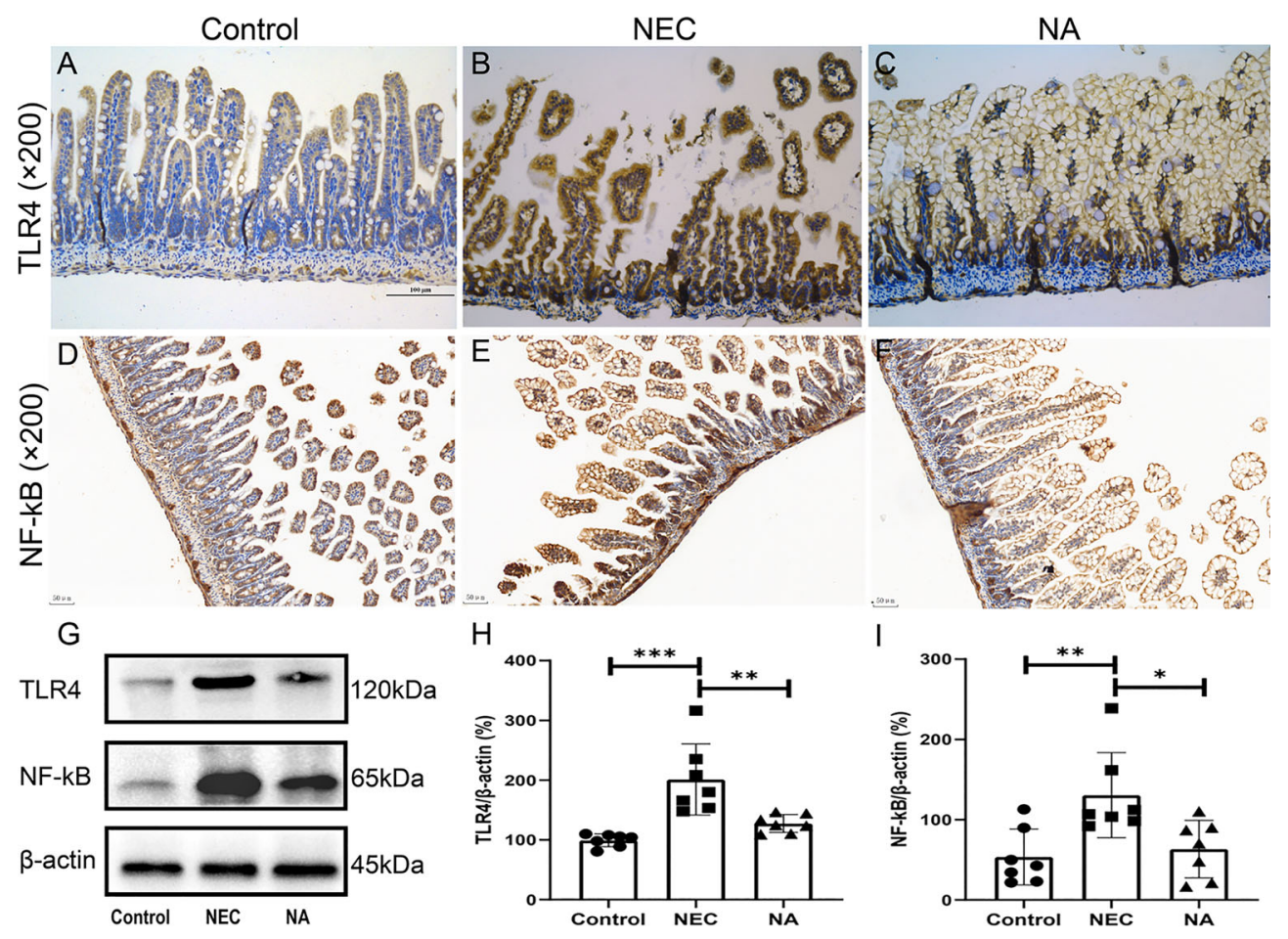

FIGURE 6 | (A-C) Expression of the TLR4 and NF-KB (D-F) proteins was assessed in intestinal tissues of the three groups by immunohistochemical staining. Magnification: $\times 200$. Scale bars $=100 \mu \mathrm{m}(T L R 4)$ and $50 \mu \mathrm{m}(\mathrm{NF}-\kappa \mathrm{B})$. Images of immunohistochemical staining of the negative control (secondary antibody and DAB control alone) are provided in Supplementary Figure 4. (G-I) Expression of the TLR4 and NF- $\kappa B$ proteins was determined in intestinal tissues of the three groups by western blotting. Numbers of samples: Con $(n=7)$, NEC $(n=7)$, and NA $(n=7)$. Statistics: Kruskal-Wallis test ${ }^{\star} P<0.05,{ }^{\star \star} P<0.01,{ }^{\star \star \star} P<0.001$.

study has shown that changes in AI-2 are associated with the NEC stage. AI-2 levels were reduced in the acute stage and increased in the recovery stage of NEC. The AI- 2 concentration inversely correlates with the degree of inflammation and microbial dysbiosis in NEC (Fu et al., 2020). In the present study, a similar phenomenon was observed in the mouse NEC model (Figure 3). The AI-2 level was significantly lower in the NEC group than in the other two groups, and intestinal microbiota dysbiosis occurred (Figures 3, 4). The addition of exogenous AI-2 increases the level of AI-2 in the intestine. In the current study, exogenous AI-2 supplementation increased the AI-2 concentration in the NEC mice (Figure 3), with a decrease in the inflammatory response. It has been reported that the disruption of the normal microbiota composition by antibiotic treatment leads to a reduction in AI-2 levels and that artificially increasing the concentration of intestinal AI-2 can attenuate the imbalance in intestinal microbiota and the expression of virulence genes (Sun et al., 2015; Thompson et al., 2015; Fu et al., 2020). Interestingly, after exogenous AI-2 supplementation in the present study, the microbiota showed a tendency to partially return to a normal status, which was consistent with the findings reported by Hsiao et al. AI-2 produced by Ruminococcus obeum can upregulate the QS system of Vibrio cholerae, disrupting its density-sensing regulatory system and leading to the expression of immature virulence factors, thereby reducing the proportion and virulence of $V$. cholerae in the intestine (Hsiao et al., 2014).

Thus, we speculate that multiple factors leading to microbiota dysbiosis significantly decrease the AI-2 concentration, which results in abnormal regulation of the QS system to maintain the stability of the intestinal microbiota, ultimately leading to the occurrence of NEC. Further, the addition of exogenous AI-2 can increase its level in the intestine, which is conducive to the regulation of intestinal homeostasis by the QS system and reduces the inflammatory response and microbiota dysbiosis.

\section{Exogenous Al-2 Might Reduce Intestinal Inflammation}

Studies have shown that the expression of TLR4 in the gut epithelium is increased in human and mouse intestinal inflammation (Leaphart et al., 2007; Egan et al., 2016), and overexpression of TLR4 leads to a signaling cascade that induces nuclear translocation of NF-kB and then promotes overtranscription of proinflammatory cytokines, then leads to the incidence of NEC (Hackam and Sodhi, 2018). Meanwhile, TLR4 inhibitors can relieve inflammation in human and animal NEC models (Niño et al., 2016; Hackam and Sodhi, 2018). In our study, we found that exogenous AI-2 supplementation may promote the expression of the anti-inflammatory factor IL-10 and decrease the expression of proinflammatory factors. 
Meanwhile, low expression of TLR4 and NF- $\mathrm{KB}$ was observed after exogenous AI-2 supplementation. However, no relevant literature to report the cause of this phenomenon in intestine. Therefore, it would be interesting to examine whether exogenous AI- 2 could reduce intestinal inflammation in a mouse NEC model by inhibiting TLR4/NF- $\kappa \mathrm{B}$ pathway.

In summary, our findings suggest that the administration of exogenous AI-2 may partially reverse the microbiota disorder and decrease inflammation in the mouse NEC model. This study provides new insight into the potential treatment strategy. Further studies are required to ascertain the precise mechanism of the AI-2 effect on NEC.

\section{DATA AVAILABILITY STATEMENT}

Our DNA sequencing data has been uploaded to the NCBI database. The BioProject accession number is PRJNA722686.

\section{ETHICS STATEMENT}

The animal study was reviewed and approved by Institutional Animal Care and Use Committee (IACUC) at the University of Chongqing Medical University.

\section{AUTHOR CONTRIBUTIONS}

L-QL and Z-LW contributed to conceptualization, funding acquisition, and supervision. Y-CJ, QS, C-YF and XS helped

\section{REFERENCES}

Baranowski, J. R., and Claud, E. C. (2019). Necrotizing Enterocolitis and the Preterm Infant Microbiome. Adv. Exp. Med. Biol. 1125, 25-36. doi: 10.1007/5584_2018_313

Bellodas Sanchez, J., and Kadrofske, M. (2019). Necrotizing Enterocolitis. Neurogastroenterol Motil. 31 (3), e13569. doi: 10.1111/nmo.13569

Buck, B. L., Azcarate-Peril, M. A., and Klaenhammer, T. R. (2009). Role of Autoinducer-2 on the Adhesion Ability of Lactobacillus Acidophilus. J. Appl. Microbiol. 107 (1), 269-279. doi: 10.1111/j.1365-2672.2009.04204.x

Cohen, T. S., and Prince, A. S. (2013). Activation of Inflammasome Signaling Mediates Pathology of Acute P. Aeruginosa Pneumonia. J. Clin. Invest. 123 (4), 1630-1637. doi: 10.1172/jci66142

Cuadra-Saenz, G., Rao, D. L., Underwood, A. J., Belapure, S. A., Campagna, S. R., Sun, Z., et al. (2012). Autoinducer-2 Influences Interactions Amongst Pioneer Colonizing Streptococci in Oral Biofilms. Microbiol. (Reading) 158 (Pt 7), 1783-1795. doi: 10.1099/mic.0.057182-0

De Keersmaecker, S. C., Varszegi, C., van Boxel, N., Habel, L. W., Metzger, K., Daniels, R., et al. (2005). Chemical Synthesis of (S)-4,5-Dihydroxy-2,3Pentanedione, A Bacterial Signal Molecule Precursor, and Validation of Its Activity in Salmonella Typhimurium. J. Biol. Chem. 280 (20), 19563-19568. doi: 10.1074/jbc.M412660200

Egan, C. E., Sodhi, C. P., Good, M., Lin, J., Jia, H., Yamaguchi, Y., et al. (2016). Toll-Like Receptor 4-Mediated Lymphocyte Influx Induces Neonatal Necrotizing Enterocolitis. J. Clin. Invest. 126 (2), 495-508. doi: 10.1172/jci83356

Frost, B. L., Modi, B. P., Jaksic, T., and Caplan, M. S. (2017). New Medical and Surgical Insights Into Neonatal Necrotizing Enterocolitis: A Review. JAMA Pediatr. 171 (1), 83-88. doi: 10.1001/jamapediatrics.2016.2708 with data curation. L-QL, Y-CJ, QS, C-YF, XS, and XC-L worked on the formal analysis. Y-CJ, QS, C-YF, L-QL, XS, and Z-LW carried out the investigation. Y-CJ, QS, C-YF, XS, Z-LW, and QA contributed to the methodology. Y-CJ, QS, C-YF and YH were responsible for project administration and writing the original draft. XS and QA contributed to resources. Z-LW was responsible for the validation. Z-LW and L-QL reviewed and edited the manuscript. All authors contributed to the article and approved the submitted version.

\section{FUNDING}

This work was supported by the Scientific Research Foundation of the Science and Technology Commission of Chongqing (Grant No. cstc2019jcyj-msxmX0169), the General Basic Research Project from the Ministry of Education Key Laboratory of Child Development and Disorders (Grant No. GBRP-202107), the Chongqing Municipal Administration of Human Resources and Social Security (Grant No. Cx2017107), the National Natural Science Foundation of China (Grant No. 82001602), and the Science and Health Project of the Chongqing Health Commission (Grant No. 2020FYYX217).

\section{SUPPLEMENTARY MATERIAL}

The Supplementary Material for this article can be found online at: https://www.frontiersin.org/articles/10.3389/fcimb.2021.694395/ full\#supplementary-material

Fu, C. Y., Li, L. Q., Yang, T., She, X., Ai, Q., and Wang, Z. L. (2020). Autoinducer-2 May Be a New Biomarker for Monitoring Neonatal Necrotizing Enterocolitis. Front. Cell Infect. Microbiol. 10, 140. doi: 10.3389/fcimb.2020.00140

Garg, P. M., Tatum, R., Ravisankar, S., Shekhawat, P. S., and Chen, Y. H. (2015). Necrotizing Enterocolitis in a Mouse Model Leads to Widespread Renal Inflammation, Acute Kidney Injury, and Disruption of Renal Tight Junction Proteins. Pediatr. Res. 78 (5), 527-532. doi: 10.1038/pr.2015.146

Gopalakrishna, K. P., Macadangdang, B. R., Rogers, M. B., Tometich, J. T., Firek, B. A., Baker, R., et al. (2019). Maternal IgA Protects Against the Development of Necrotizing Enterocolitis in Preterm Infants. Nat. Med. 25 (7), 1110-1115. doi: 10.1038/s41591-019-0480-9

Hackam, D. J., and Sodhi, C. P. (2018). Toll-Like Receptor-Mediated Intestinal Inflammatory Imbalance in the Pathogenesis of Necrotizing Enterocolitis. Cell Mol. Gastroenterol. Hepatol 6 (2), 229-238.e221. doi: 10.1016/j.jcmgh. 2018.04.001

Hinde, K., and Lewis, Z. T. (2015). MICROBIOTA. Mother's Littlest Helpers. Science 348 (6242), 1427-1428. doi: 10.1126/science.aac7436

Hsiao, A., Ahmed, A. M., Subramanian, S., Griffin, N. W., Drewry, L. L., Petri, W. A. Jr., et al. (2014). Members of the Human Gut Microbiota Involved in Recovery From Vibrio Cholerae Infection. Nature 515 (7527), 423-426. doi: 10.1038/nature13738

Isani, M. A., Delaplain, P. T., Grishin, A., and Ford, H. R. (2018). Evolving Understanding of Neonatal Necrotizing Enterocolitis. Curr. Opin. Pediatr. 30 (3), 417-423. doi: 10.1097/mop.0000000000000629

Kawai, T., and Akira, S. (2010). The Role of Pattern-Recognition Receptors in Innate Immunity: Update on Toll-Like Receptors. Nat. Immunol. 11 (5), 373 384. doi: $10.1038 /$ ni.1863 
Leaphart, C. L., Cavallo, J., Gribar, S. C., Cetin, S., Li, J., Branca, M. F., et al. (2007). A Critical Role for TLR4 in the Pathogenesis of Necrotizing Enterocolitis by Modulating Intestinal Injury and Repair. J. Immunol. 179 (7), 4808-4820. doi: 10.4049/jimmunol.179.7.4808

Mihi, B., and Good, M. (2019). Impact of Toll-Like Receptor 4 Signaling in Necrotizing Enterocolitis: The State of the Science. Clin. Perinatol 46 (1), 145157. doi: 10.1016/j.clp.2018.09.007

Neu, J., and Walker, W. A. (2011). Necrotizing Enterocolitis. N. Engl. J. Med. 364 (3), 255-264. doi: 10.1056/NEJMra1005408

Niemarkt, H. J., de Meij, T. G., van de Velde, M. E., van der Schee, M. P., van Goudoever, J. B., Kramer, B. W., et al. (2015). Necrotizing Enterocolitis: A Clinical Review on Diagnostic Biomarkers and the Role of the Intestinal Microbiota. Inflamm. Bowel Dis. 21 (2), 436-444. doi: 10.1097/mib. 0000000000000184

Niño, D. F., Sodhi, C. P., and Hackam, D. J. (2016). Necrotizing Enterocolitis: New Insights Into Pathogenesis and Mechanisms. Nat. Rev. Gastroenterol. Hepatol 13 (10), 590-600. doi: 10.1038/nrgastro.2016.119

Pammi, M., Cope, J., Tarr, P. I., Warner, B. B., Morrow, A. L., Mai, V., et al. (2017). Intestinal Dysbiosis in Preterm Infants Preceding Necrotizing Enterocolitis: A Systematic Review and Meta-Analysis. Microbiome 5 (1), 31. doi: 10.1186/ s40168-017-0248-8

Park, B. S., and Lee, J. O. (2013). Recognition of Lipopolysaccharide Pattern by TLR4 Complexes. Exp. Mol. Med. 45 (12), e66. doi: 10.1038/emm.2013.97

Pereira, C. S., Thompson, J. A., and Xavier, K. B. (2013). AI-2-Mediated Signalling in Bacteria. FEMS Microbiol. Rev. 37 (2), 156-181. doi: 10.1111/j.1574-6976.2012.00345.x

Raut, N., Pasini, P., and Daunert, S. (2013). Deciphering Bacterial Universal Language by Detecting the Quorum Sensing Signal, Autoinducer-2, With a Whole-Cell Sensing System. Anal. Chem. 85 (20), 9604-9609. doi: 10.1021/ac401776k

Raveh-Sadka, T., Thomas, B. C., Singh, A., Firek, B., Brooks, B., Castelle, C. J., et al. (2015). Gut Bacteria are Rarely Shared by Co-Hospitalized Premature Infants, Regardless of Necrotizing Enterocolitis Development. Elife 4, e05477. doi: 10.7554/eLife.05477

Robertson, C., Savva, G. M., Clapuci, R., Jones, J., Maimouni, H., Brown, E., et al. (2020). Incidence of Necrotising Enterocolitis Before and After Introducing Routine Prophylactic Lactobacillus and Bifidobacterium Probiotics. Arch. Dis. Child Fetal Neonatal Ed 105 (4), 380-386. doi: 10.1136/archdischild-2019-317346

Schönherr-Hellec, S., and Aires, J. (2019). Clostridia and Necrotizing Enterocolitis in Preterm Neonates. Anaerobe 58, 6-12. doi: 10.1016/j.anaerobe.2019.04.005

Shi, N., Li, N., Duan, X., and Niu, H. (2017). Interaction Between the Gut Microbiome and Mucosal Immune System. Mil Med. Res. 4, 14. doi: 10.1186/s40779-017-0122-9

Shulhan, J., Dicken, B., Hartling, L., and Larsen, B. M. (2017). Current Knowledge of Necrotizing Enterocolitis in Preterm Infants and the Impact of Different
Types of Enteral Nutrition Products. Adv. Nutr. 8 (1), 80-91. doi: 10.3945/ an.116.013193

Sun, Z., Grimm, V., and Riedel, C. U. (2015). AI-2 to the Rescue Against Antibiotic-Induced Intestinal Dysbiosis? Trends Microbiol. 23 (6), 327-328. doi: 10.1016/j.tim.2015.04.002

Thompson, J. A., Oliveira, R. A., Djukovic, A., Ubeda, C., and Xavier, K. B. (2015). Manipulation of the Quorum Sensing Signal AI-2 Affects the AntibioticTreated Gut Microbiota. Cell Rep. 10 (11), 1861-1871. doi: 10.1016/ j.celrep.2015.02.049

Van Belkum, M., Mendoza Alvarez, L., and Neu, J. (2020). Preterm Neonatal Immunology at the Intestinal Interface. Cell Mol. Life Sci. 77 (7), 1209-1227. doi: 10.1007/s00018-019-03316-w

Wang, Y., Liu, B., Grenier, D., and Yi, L. (2019). Regulatory Mechanisms of the LuxS/AI-2 System and Bacterial Resistance. Antimicrob. Agents Chemother. 63 (10), e01186-19. doi: 10.1128/aac.01186-19

Wu, S., Liu, J., Liu, C., Yang, A., and Qiao, J. (2020). Quorum Sensing for Population-Level Control of Bacteria and Potential Therapeutic Applications. Cell Mol. Life Sci. 77 (7), 1319-1343. doi: 10.1007/s00018-019-03326-8

Xiao, S., Li, Q., Hu, K., He, Y., Ai, Q., Hu, L., et al. (2018). Vitamin A and Retinoic Acid Exhibit Protective Effects on Necrotizing Enterocolitis by Regulating Intestinal Flora and Enhancing the Intestinal Epithelial Barrier. Arch. Med. Res. 49 (1), 1-9. doi: 10.1016/j.arcmed.2018.04.003

Yu, X., Radulescu, A., Zorko, N., and Besner, G. E. (2009). Heparin-Binding EGFLike Growth Factor Increases Intestinal Microvascular Blood Flow in Necrotizing Enterocolitis. Gastroenterology 137 (1), 221-230. doi: 10.1053/ j.gastro.2009.03.060

Conflict of Interest: The authors declare that the research was conducted in the absence of any commercial or financial relationships that could be construed as a potential conflict of interest.

Publisher's Note: All claims expressed in this article are solely those of the authors and do not necessarily represent those of their affiliated organizations, or those of the publisher, the editors and the reviewers. Any product that may be evaluated in this article, or claim that may be made by its manufacturer, is not guaranteed or endorsed by the publisher.

Copyright (c) $2021 \mathrm{Ji}$, Sun, Fu, She, Liu, He, Ai, Li and Wang. This is an open-access article distributed under the terms of the Creative Commons Attribution License (CC BY). The use, distribution or reproduction in other forums is permitted, provided the original author(s) and the copyright owner(s) are credited and that the original publication in this journal is cited, in accordance with accepted academic practice. No use, distribution or reproduction is permitted which does not comply with these terms. 


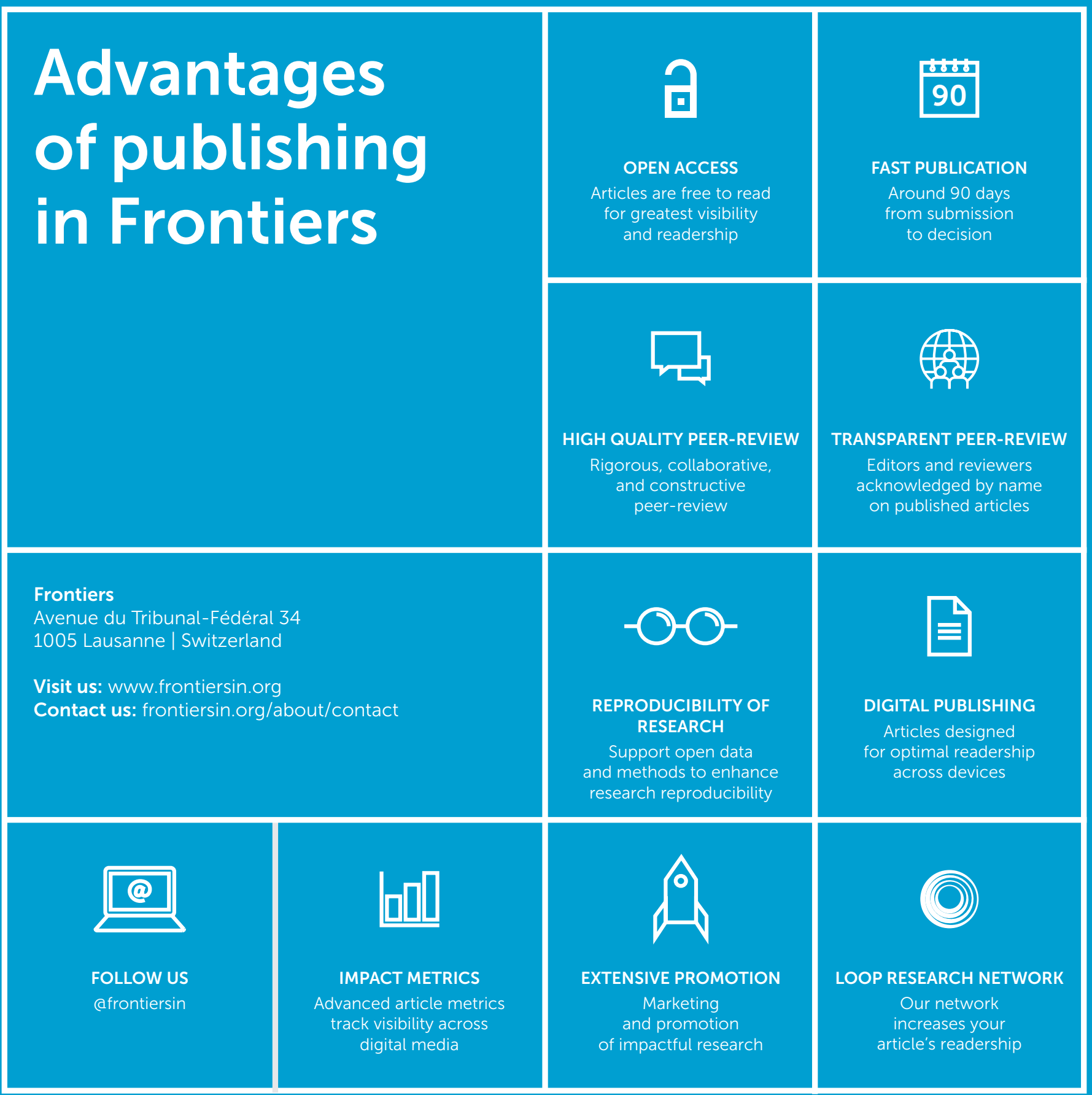

